\title{
A new phylogenetic hypothesis of Tanystropheidae (Diapsida, Archosauromorpha) and other "protorosaurs", and its implications for the early evolution of stem archosaurs
}

\author{
Stephan N F Spiekman ${ }^{\text {Corresp., } 1 \text {, Nicholas C Fraser }}{ }^{2}$, Torsten M Scheyer $^{1}$ \\ 1 Palaeontological Institute and Museum, University of Zurich, Zurich, Switzerland \\ 2 National Museums Scotland, Edinburgh, United Kingdom \\ Corresponding Author: Stephan N F Spiekman \\ Email address: stephanspiekman@gmail.com
}

The historical clade "Protorosauria" represents an important group of archosauromorph reptiles that had a wide geographic distribution between the Late Permian and Late Triassic. "Protorosaurs" are characterized by their long necks, which are epitomized in the genus Tanystropheus and in Dinocephalosaurus orientalis. Recent phylogenetic analyses have indicated that "Protorosauria" is a polyphyletic clade, but the exact relationships of the various "protorosaur" taxa within the archosauromorph lineage is currently uncertain. Several taxa, although represented by relatively complete material, have previously not been assessed phylogenetically. We present a new phylogenetic hypothesis that comprises a wide range of archosauromorphs, including the most exhaustive sample of "protorosaurs" to date and several "protorosaur" taxa from the eastern Tethys margin that have not been included in any previous analysis. The polyphyly of "Protorosauria" is confirmed and therefore we suggest the usage of this term should be abandoned.

Tanystropheidae is recovered as a monophyletic group and the Chinese taxa Dinocephalosaurus orientalis and Pectodens zhenyuensis form a new archosauromorph clade, Dinocephalosauridae, which is closely related to Tanystropheidae. The well-known crocopod and former "protorosaur" Prolacerta broomi is considerably less closely related to Archosauriformes than was previously considered. 
1 A new phylogenetic hypothesis of Tanystropheidae (Diapsida, Archosauromorpha) and other

2 "protorosaurs", and its implications for the early evolution of stem archosaurs

3 Stephan N. F. Spiekman ${ }^{1}$, Nicholas C. Fraser ${ }^{2}$, Torsten M. Scheyer ${ }^{1}$

4

5 ' ${ }^{1}$ niversity of Zurich, Palaeontological Institute and Museum, Karl-Schmid-Strasse 4, 8006 Zurich,

6 Switzerland

$7 \quad{ }^{2}$ National Museums Scotland, Chambers St, Edinburgh EH1 1JF, UK

8

9 Corresponding author:

10 Stephan Spiekman

11 Karl-Schmid-Strasse 4, 8006 Zurich, Switzerland

12 Email address: stephanspiekman@gmail.com

\section{Abstract}

The historical clade "Protorosauria" represents an important group of archosauromorph reptiles that had a wide geographic distribution between the late Permian and Late Triassic. "Protorosaurs" are characterized by their long necks, which are epitomized in the genera Tanystropheus and Dinocephalosaurus. Recent phylogenetic analyses have indicated that "Protorosauria" is a polyphyletic clade, but the exact relationships of the various "protorosaur" taxa within the archosauromorph lineage remains unclear. Several taxa, although represented by relatively complete material, have previously not been assessed phylogenetically. We provide a historical overview of all taxa formerly considered as "protorosaurs" and present a new phylogenetic hypothesis that comprises a wide range of nonarchosauriform archosauromorphs with the most exhaustive sample of "protorosaurs" to date, including several recently described taxa from the eastern Tethys margin in modern China. The polyphyly of "Protorosauria" is confirmed. Tanystropheidae is recovered as a monophyletic group and the Chinese taxa Dinocephalosaurus orientalis and Pectodens zhenyuensis form a new non-archosauriform archosauromorph clade, Dinocephalosauridae. The well-known former "protorosaur" Prolacerta broomi 
28 is considerably less closely related to Archosauriformes than was previously considered and might not be 29 referrable to Crocopoda.

\section{Introduction}

Non-archosauriform archosauromorphs lived during the late Permian and Triassic and belong to the archosaurian stem-lineage, the ancestral lineage of crocodylians and birds. Historically, many members of this group were placed within either "Protorosauria" or "Prolacertiformes". These two groups generally encompassed the same taxa and the usage of one term over the other depended on the inclusion within the clade of either Protorosaurus speneri or Prolacerta broomi, or both. Since both names generally apply to the same taxa and are often used interchangeably, and because "Protorosauria" Huxley 1871 predates "Prolacertiformes" Camp 1945, we refer to the members of these groups here as "Protorosauria" (sensu Chatterjee 1986). Apart from the two above mentioned taxa, the terrestrial and aquatic long-necked tanystropheids (e.g., Tanystropheus, Macrocnemus, Langobardisaurus, and Tanytrachelos) represent the most morphologically diverse and best-known members of "Protorosauria". Formerly, the enigmatic arboreal drepanosaurids were also referred to the clade, but they have recently been revealed to represent a separate clade of non-saurian diapsids (Pritchard \& Nesbitt 2017; Pritchard et al. 2016). As Permo-Triassic non-archosauriform archosauromorphs, "protorosaurs" represent some of the earliest members of the lineage that gave rise to Archosauria and as such are important both for our understanding of early archosauromorph evolution and the acquisition of traits within the archosaur character complex. For instance, the Chinese Dinocephalosaurus orientalis and an unnamed closely related taxon represent the only known viviparous archosauromorphs (Li et al. 2017b; Liu et al. 2017).

Recent cladistic studies have extensively dealt with early archosauromorph phylogeny (early Archosauria, Nesbitt 2011; early Archosauromorpha with a focus on proterosuchians, Ezcurra 2016; Allokotosauria, Nesbitt et al. 2015; Rhynchosauria, Butler et al. 2015 and Ezcurra et al. 2016; and Tanystropheidae, Pritchard et al. 2015). These, and some earlier analyses, indicate that "Protorosauria" does not form a monophyletic clade as historically considered, but rather represents a paraphyletic or polyphyletic grouping of non-archosauriform archosauromorphs (Fig. 1, but for an exception see Simões et al. 2018, who recovered Protorosauria excluding Prolacerta as a monophyletic clade outside Archosauromorpha). However, none of these analyses were constructed to specifically address the interrelationships of "Protorosauria" and many recently described taxa (e.g., the genera Pectodens, Fuyuansaurus, Dinocephalosaurus, Raibliania, Elessaurus, and Sclerostropheus) attributed to the group or an equivalent grade in the archosauromorph tree have not been included (Dalla Vecchia 2020; De Oliveira 
et al. 2020; Fraser et al. 2013; Li et al. 2017a; Rieppel et al. 2008; Spiekman \& Scheyer 2019). Moreover, the two best-known tanystropheid genera, Tanystropheus and Macrocnemus, were recently revised extensively, revealing much additional morphological information, particular with regards to the skull, which has not been incorporated in the abovementioned analyses (Miedema et al. 2020; Spiekman et al. 2020a; Spiekman et al. 2020b).

Here we present an extensive phylogenetic analysis, focusing on "protorosaur" and other early archosauromorph interrelationships. The new dataset includes 42 operational taxonomic units (OTUs), of which 23 are "protorosaurs", and employs 307 morphological characters, many of which are new or distinctly revised from previous analyses. Since the definition of "Protorosauria" in the literature is inconsistent, with many taxa having been placed alternately within and outside the group, we first provide a historical overview of "protorosaur" systematics and discuss the taxa that have formerly been included in the group. Several of these are represented by very fragmentary material or have since been identified as belonging to an entirely separate lineage to that of the archosauromorph "protorosaurs", and they were therefore not included in our phylogenetic analysis.

Historical background of "Protorosauria"

Protorosaurus speneri is one of the earliest known fossil reptiles, first described in Latin by Spener (1710). He considered Protorosaurus to be a crocodile, with many similarities specifically to the Nile crocodile, Crocodylus niloticus (Gottmann-Quesada \& Sander 2009). More than a century later, Protorosaurus was recognized as an extinct reptile (Meyer 1830), and subsequently assigned a species definition (Meyer 1832) and covered in an extensive monograph (Meyer 1856). The clade "Protorosauria", with Protorosaurus as the only representative, was erected by Huxley (1871), as part of Sauropsida, then as now considered to be the clade that encompasses all modern birds and reptiles and their direct ancestors. In his classification of the reptiles, Osborn (1903) provided the first definition of "Protorosauria" and assigned Palaeohatteria, a synapsid (Fröbisch et al. 2011), and Kadaliosaurus, an araeoscelid diapsid (DeBraga \& Reisz 1995), to the clade. Therein, the group was closely related to dinosaurs. Williston (1925) placed "protorosaurs" within "Parapsida" alongside squamates, ichthyosaurs, and mesosaurs. Other genera that were included within "Protorosauria" were Sapheosaurus and Pleurosaurus, now firmly established rhynchocephalians (Hsiou et al. 2019; Rauhut et al. 2012), and Araeoscelis and Aphelosaurus, now considered to be non-neodiapsid diapsids (Ezcurra et al. 2014; Reisz et al. 2011). 
After extensive excavations at the Anisian-Ladinian deposits of Monte San Giorgio on the border between Switzerland and Italy, newly discovered specimens allowed for the first comprehensive description of both Tanystropheus longobardicus and Macrocnemus bassanii (Peyer 1931; Peyer 1937). Initially Tanystropheus longobardicus was placed within a newly erected suborder "Tanysitrachelia", which apart from Tanystropheus also included Trachelosaurus fischeri, a small, long-necked reptile from the Buntsandstein (Early to Middle Triassic) of Germany (Broili \& Fischer 1918). "Tanysitrachelia" was placed within Sauropterygia (Peyer 1931). Trachelosaurus is only known from a few disarticulated postcranial elements and therefore its phylogenetic position is uncertain, although it is currently still considered a "protorosaur" (Benton \& Allen 1997; Jalil 1997; Rieppel et al. 2003). However, in the later report on Macrocnemus bassanii, Peyer (1937) found many similarities between Protorosaurus and both Macrocnemus and Tanystropheus, and therefore both taxa were reassigned to "Protorosauria", which was considered closely related to squamates and rhynchocephalians rather than archosaurs therein.

Around the same time Prolacerta broomi was described and assigned to the newly erected family "Prolacertidae" (Parrington 1935). "Prolacertidae" was classified as part of "Thecodontia", a group that was at the time considered either as a "primitive" lineage within Archosauria (Watson 1917), or ancestral to both archosaurs and lepidosaurs (Broom 1914). "Thecodontia" is now unequivocally a paraphyletic grouping and has been abandoned as a clade (Benton 2005). However, based on its incomplete infratemporal bar, Prolacerta was considered to be intermediate between "lacertilians" (i.e., squamates) and more "primitive thecodonts" such as Youngina capensis (Parrington 1935). The description of a new specimen of Prolacerta led to the consideration that it was more closely related to Protorosaurus and resulted in the first inclusion of Prolacerta into "Protorosauria" (Camp 1945). Camp (1945) favored "Protorosauria" over "Eosuchia" based on seniority, and included taxa placed in "Eosuchia", "Trachelosauria", and "Protorosauria" by Williston (1925) within this clade and established it within Lepidosauria. This superorder "Protorosauria" was further subdivided in the orders

"Prolacertiformes", which he synonymized with "Eosuchia" (sensu Broom 1914, meaning it also included "Younginiformes"), "Trachelosauria" or Tanystropheidae, and, more tentatively, Thalattosauria and "Acrosauria" (the latter containing the rhynchocephalian pleurosaurids).

Kuhn-Schnyder (1954) defined the Middle Triassic Macrocnemus and Tanystropheus as squamates (German: Eidechsen, which literally translates to lacertids) that were morphologically intermediate between the Jurassic squamates and the supposed 'squamate ancestor' Prolacerta. Protorosaurus was not considered, since this interpretation was based mainly on skull anatomy, which 
120 was insufficiently understood in Protorosaurus at this point. This hypothesis differed from that of 121 Colbert $(1945 ; 1965)$ and Romer $(1956 ; 1966 ; 1968)$, who considered "protorosaurs" as "Euryapsida" 122 (sometimes also called "Synaptosauria"; Cope 1900), a clade which consisted of "protorosaurs" and 123 sauropterygians, thus being similar to Sauropterygia as defined previously by Peyer (1931). This 124 classification, which represented an important systematic paradigm for reptiles, was largely based on 125 the temporal fenestration of the skull. "Euryapsids" were considered as a group that was entirely 126 separate from "anapsids", synapsids, and diapsids, based on the presence of an upper temporal fenestra 127 surrounded by the postorbital, squamosal, and parietal, and the absence of a lower temporal fenestra.

128 The inclusion of "protorosaurs" within "euryapsids" was mainly based on Araeoscelis, which shows this 129 fenestration type, in contrast to other "protorosaurs" that show the typical diapsid condition. Among others, the "Protorosauria" of Romer (1966) included Protorosaurus, Tanystropheus, Trachelosaurus, and Trilophosaurus (the last taxon is currently considered an allokotosaur within non-archosauriform Archosauromorpha; Ezcurra 2016; Nesbitt et al. 2015; Sengupta et al. 2017). On the other hand, Prolacerta and Macrocnemus were assigned to "Prolacertiformes" within "Eosuchia", interpreted as the 'basalmost' order of Lepidosauria ("Eosuchia" was maintained contra Camp 1945). "Euryapsida" has generally not been used as a grouping in recent years, and its former members are now distributed within Diapsida (Benton 2005; Merck 1997). Furthermore, an extensive redescription of Araeoscelis has shown various differences with taxa such as Protorosaurus and Prolacerta (Vaughn 1955), and it is now considered an early diapsid that it is not closely related to "protorosaurs" (Ford \& Benson 2020). The hypothesis of "Euryapsida" comprised of Sauropterygia and "Protorosauria" was criticized by KuhnSchnyder (1963; 1967; 1974). Kuhn-Schnyder (1967) and Wild (1973) argued that because of the ventrally opened lower temporal bar of Macrocnemus and Tanystropheus, "Protorosauria", including Protorosaurus, belonged to "Prolacertidae" within Lepidosauria. the first detailed description of postcranial remains. This study was the first to conclude that Prolacerta, together with Macrocnemus and Tanystropheus, was clearly not part of the lepidosaurian lineage, but instead was archosaurian in many of its features. These taxa were grouped in the newly erected order "Parathecodontia", with Prolacerta and Macrocnemus being further classified together within "Prolacertidae" and Tanystropheus within Tanystropheidae. Nevertheless, the need for a detailed phylogenetic re-examination of these taxa was stressed and this revision did not consider Protorosaurus. 
In the 1970s and the subsequent two decades, a considerable number of taxa were included

151

152

153

154

155

156

157

158

159

160

161

162

163

164

165

166

167

168

169

170

171

172

173

174

175

176

177

178

179

180

within "Protorosauria", further indicating the significance of the group: Tanytrachelos ahynis (Olsen 1979), Langobardisaurus pandolfii (Bizzarini \& Muscio 1995), Cosesaurus aviceps (originally considered an avian ancestor; Ellenberger \& De Villalta 1974, but later designated as a "protorosaur" by Olsen 1979), Malerisaurus robinsonae (Chatterjee 1980), Kadimakara australiensis (Bartholomai 1979), Prolacertoides jimusarensis (Young 1973), Malutinisuchus gratus (Ochev 1986), and Boreopricea funerea (Tatarinov 1978). In addition, "Protorosauria" as designated by Evans (1988) included Megalancosaurus preonensis, a member of the Drepanosauridae, a family of highly specialized, arboreal diapsids (Calzavara et al. 1980; Pritchard et al. 2016; Renesto et al. 2010). Chatterjee (1980) also included the Carboniferous Petrolacosaurus kansensis within "Prolacertiformes", although this view was swiftly disputed (Evans 1988; Reisz et al. 1984), and Petrolacosaurus is now widely considered an araeoscelid diapsid instead (Ezcurra et al. 2014; Ford \& Benson 2020; Reisz et al. 2011).

Cladistics became widespread as a method for establishing phylogenetic relationships between taxa during the 1980s and its implementation on diapsid phylogeny quickly led to a relatively clear-cut division between Lepidosauromorpha and Archosauromorpha, with "Protorosauria" firmly established within the latter group (Bennett 1996; Benton 1984; Benton 1985; Evans 1988; Gauthier 1984; Gauthier 1994; Gauthier et al. 1988b). Chatterjee (1986) pointed out the priority of "Protorosauria" over "Prolacertiformes" based on seniority, but since "Protorosauria" had previously often included Araeoscelis and was therefore shown to be polyphyletic, many authors since preferred "Prolacertiformes" (see Evans 1988, pages 226-227 for an overview of the use of both terms within the literature between 1945 and 1988). However, although the place of "protorosaurs" among Archosauromorpha became firmly established, the interrelationships of the various "protorosaurs" was not evaluated cladistically except by Chatterjee (1986) and Evans (1988). Olsen (1979) and Wild (1980a) also provided a hypothesis of "protorosaur" interrelationships on a non-cladistic basis.

This issue would soon be addressed in more detail in several papers. One study included 11 "protorosaurs" (excluding the poorly known Prolacertoides) and three outgroups and 48 morphological characters (Benton \& Allen 1997). In the same year, the description of a new "protorosaur", Jesairosaurus lehmani, was accompanied by an analysis including ten "protorosaurs" and eight outgroup taxa, employing 71 characters (Jalil 1997; the initial analysis also included Trachelosaurus, Prolacertoides, Malutinisuchus, and Kadimakara, but these poorly known taxa were excluded from the final analysis, as the inclusion of these taxa left "protorosaurs" unresolved). Another study addressing 
181 early archosauromorph phylogeny also included several "protorosaurs" (Dilkes 1998). This analysis

182 included 144 characters and 23 taxa, out of which seven were traditionally considered as

183 "protorosaurs", including two drepanosaurid taxa, which were not included in Benton \& Allen (1997)

184 and Jalil (1997). It recovered a monophyletic "Protorosauria" in which Protorosaurus formed a sister

185 taxon to two lineages, Drepanosauridae and Tanystropheidae, whereas Prolacerta was placed outside

186 the clade as the sister taxon of Archosauriformes. Peters (2000) used the matrices of Evans (1988), Jalil

187 (1997), and Bennett (1996) and reran each of them after adding a number of characters and rescoring

188 some characters for certain taxa, for a total taxon sample that included 11 "protorosaurs", other non-

189 archosauriform archosauromorphs, the pterosaur Eudimorphodon, and two enigmatic and possibly

190 gliding diapsids, Longisquama insignis (Sharov 1970) and Sharovipteryx mirabilis (Cowen 1981; Sharov

191 1971). Sharovipteryx is an enigmatic gliding reptile with a membrane stretched between the hindlimbs,

192 which represents an entirely unique morphology among gliding reptiles. It has been tentatively ascribed

193 to "protorosaurs" or tanystropheids by some authors (Gans et al. 1987; Pritchard \& Sues 2019; Tatarinov

194 1989; Tatarinov 1994; Unwin et al. 2000), but its phylogenetic position is highly uncertain due to its

195 highly specialized, yet very poorly known morphology. Peters (2000) found "protorosaurs", and

196 Longisquama and Sharovipteryx, to be very closely associated with Eudimorphodon, from which a

197 "protorosaurian" ancestry for pterosaurs was concluded. However, the exact topologies varied strongly

198 between the different analyses, and this hypothesis of pterosaur ancestry has widely been rejected by

199 otherphylogenetic studies on pterosaurs and early archosaurs (e.g., Ezcurra 2016; Ezcurra et al. 2020;

200 Hone \& Benton 2007; Nesbitt 2011; Padian 1997). The datasets of Benton \& Allen (1997), Dilkes (1998),

201 and Jalil (1997) were combined into one larger character list of 239 characters by Rieppel et al. (2003),

202 which was used specifically to address "protorosaur" phylogeny, and in particular the question of

203 "protorosaur" monophyly, which had now been put in doubt (Dilkes 1998). This approach included

204 seven "protorosaur" taxa (Protorosaurus, Drepanosaurus, Megalancosaurus, Prolacerta, Macrocnemus,

205 Langobardisaurus, and Tanystropheus longobardicus), and four outgroup taxa (Petrolacosaurus,

206 Youngina, Rhynchosaurus, and Trilophosaurus). Additional analyses were performed after subsequently

207 including Euparkeria and Proterosuchus, and the lesser known "protorosaurs" Boreopricea and

208 Jesairosaurus. Although the first analysis found a monophyletic "Protorosauria", the other two resulted

209 in paraphyly for the group. Although Rieppel et al. (2003) concluded that the monophyly of

210 "Protorosauria" as previously regarded (e.g., Benton \& Allen 1997; Jalil 1997) could not be maintained,

211 they argued the need for an extensive phylogenetic investigation into "protorosaurs". Senter (2004)

212 investigated the phylogenetic position of drepanosaurids in an analysis that comprised "protorosaurs" 
213 (Prolacerta, Macrocnemus, and Langobardisaurus), Longisquama, non-archosaurian Archosauriformes,

214 birds, a non-avian dinosaur, and a number of early diapsids. This study found drepanosaurids to form a

215 clade with Longisquama and Coelurosauravus, which was termed "Avicephala", as the sister group to

216 Neodiapsida, which in his analysis encompassed Youngina, the rhynchocephalian Gephyrosaurus, and

217 several archosauromorphs. The included "protorosaurs" formed a monophyletic clade within

218 Archosauromorpha. However, an analysis using the same character list by Renesto \& Binelli (2006) could

219 not reproduce the same topology. Renesto et al. (2010) reaffirmed the position of drepanosaurids

220 among "protorosaurs", whereas Pritchard \& Nesbitt (2017) recovered Drepanosauromorpha as a

221 separate clade of non-saurian diapsids. Müller (2004) included four different "protorosaur" taxa in his

222 broad-scale analysis of diapsid relationships, which consisted of 184 characters compiled mainly from

223 Rieppel et al. (1999) and DeBraga \& Rieppel (1997). This study also inferred a polyphyletic

224 "Protorosauria", with Tanystropheus, Macrocnemus, and Prolacerta being successive sister taxa to

225 rhynchosaurs and Trilophosaurus, whereas drepanosaurids were only quite distantly related to these

226 taxa.

227

"Protorosaurs" were virtually unknown from China until about 15 years ago, with the exception

228 of the poorly known and tentative "protorosaur" Prolacertoides jimusarensis (Young 1973). However, a

229 number of new finds have been referred to "Protorosauria", including Tanystropheus cf. longobardicus

230 (Rieppel et al. 2010; now Tanystropheus cf. hydroides, see Spiekman et al. 2020a), Tanystropheus sp. (Li

231 2007), and Macrocnemus fuyuanensis (Jiang et al. 2011; Li et al. 2007), forms very similar to European

232 counterparts, as well as completely new taxa, such as Dinocephalosaurus orientalis (Li 2003; Li et al.

233 2004; Liu et al. 2017; Rieppel et al. 2008), Fuyuansaurus acutirostris (Fraser et al. 2013), an unnamed

234 taxon closely related to Dinocephalosaurus (Li et al. 2017b), and potentially Pectodens zhenyuensis (Li et

235 al. 2017a). This has revealed that "protorosaurs" had a Tethys-wide distribution and are considerably

236 more morphologically diverse than previously appreciated. Except for Dinocephalosaurus orientalis,

237 which has been included in phylogenetic analyses of Rieppel et al. (2008), Liu et al. (2017), and De

238 Oliveira et al. (2020), none of the Chinese taxa have been phylogenetically assessed so far except for the

239 matrix of Ezcurra \& Butler (2018). However, the aim of this analysis was not to investigate the

240 phylogenetic relationships between the included taxa, but rather to serve as a discrete character matrix

241 to investigate their morphological disparity.

242 But new "protorosaur" findings have also been reported from outside of China. Fraser \& Rieppel

243 (2006) re-examined the "Tanystropheus antiquus" material from the Upper Buntsandstein of Baden-

Peer] reviewing PDF | (2020:08:51657:1:1:NEW 28 Feb 2021) 
244 Württemberg, Germany, and assigned it to a new taxon, Amotosaurus rotfeldensis. Furthermore,

245 Gottmann-Quesada \& Sander (2009) provided a monograph on the German Protorosaurus speneri

246 material, including the first detailed description and reconstruction of the skull, based on the discovery

247 of a well-preserved skull in 1972, which previously had only been briefly documented (see Haubold \&

248 Schaumberg 1985 p. 223; Fichter 1995 and references therein). Gottmann-Quesada \& Sander (2009)

249 also provided a phylogenetic analysis, which employed the matrix of Dilkes (1998), with several

250 modifications to the character scorings of Mesosuchus, Prolacerta, and Protorosaurus. This resulted in a

251 tree with a polyphyletic "Protorosauria" that recovered Protorosaurus as the sister taxon to

252 Megalancosaurus. A new species of Macrocnemus, Macrocnemus obristi, has been described from

253 Alpine Europe (Fraser \& Furrer 2013), and a specimen from Monte San Giorgio on the border of

254 Switzerland and Italy was recently assigned to Macrocnemus fuyuanensis, a species that was previously

255 only known from China (Jaquier et al. 2017; Scheyer et al. 2020b). A new species of Tanystropheus,

256 Tanystropheus hydroides, has also been described from Monte San Giorgio (Spiekman et al. 2020a). This

257 new species was previously considered to represent the adult stage of Tanystropheus longobardicus

258 (Wild 1973), but long bone histology revealed that the small-sized specimens of Tanystropheus

259 longobardicus were skeletally mature, thus representing a separate species from Tanystropheus

260 hydroides. Two new "protorosaurs" have been reported from Russia based on limited, isolated remains:

261 the large-sized Vritramimosaurus dzerzhinskii, considered to be closely related to Prolacerta (Sennikov

262 2005), and Augustaburiania vatagini, a medium-sized tanystropheid (Sennikov 2011). From Poland two

263 new, possibly "protorosaur", archosauromorphs have been described. Czatkowiella harae has been

264 interpreted as being closely related to Protorosaurus (Borsuk-Białynicka \& Evans 2009b), whereas the

265 highly gracile, and putative glider, Ozimek volans is similar to Sharovipteryx (Dzik \& Sulej 2016). Ezcurra

266 et al. (2014) re-examined material consisting of five vertebrae, three fragmented forelimb elements, and

267 some indeterminable fragments from the late Permian of Tanzania previously described by Parrington

268 (1956) and assigned it to the new taxon Aenigmastropheus. Following an analysis modified from Reisz et

269 al. (2010), used to address both synapsid and diapsid relationships, it was recovered among

270 "protorosaurs" as the sister taxon to Protorosaurus. In addition, they found Eorasaurus, previously

271 assigned as a "protorosaur" by Sennikov (1997), to likely be an archosauriform, which would make

272 Aenigmastropheus the second known "protorosaur" and non-archosauriform archosauromorph from

273 the Permian, the other being Protorosaurus. Two more tanystropheid genera, Sclerostropheus fossai and

274 Raibliania calligarisi were recently identified, based on partial postcranial remains (Dalla Vecchia 2020;

275 Spiekman \& Scheyer 2019). Finally, recent findings have shone light on the occurrence and distribution

Peer] reviewing PDF | (2020:08:51657:1:1:NEW 28 Feb 2021) 
276 of tanystropheids in the Americas. Isolated material from the Middle and Late Triassic of North America,

277 largely consisting of cervical vertebrae, as well as some other postcranial remains, indicate that

278 tanystropheids were more widespread and also occurred until more recently than previously thought

279 (Formoso et al. 2019; Lessner et al. 2018; Pritchard et al. 2015; Sues \& Olsen 2015). From South

280 America, archosauromorph remains from the Induan to early Olenekian of Brazil have been described,

281 and a new species, Elessaurus gondwanoccidens, was recovered as the sister taxon to Tanystropheidae

282 (De Oliveira et al. 2018; De Oliveira et al. 2020). If this South American material is referrable to

283 Tanystropheidae, it would represent among the earliest records of the clade, and would indicate a wide,

284 if not nearly cosmopolitan distribution of the clade during the Early Triassic. In addition, material with

285 possibly "protorosaur" affinities are also known from the Permo-Triassic Buena Vista Formation of

286 northeastern Uruguay (Ezcurra et al. 2015b).

287 The original phylogenetic matrices by Pritchard et al. (2015) and Ezcurra (2016), and their

288 subsequently modified iterations (e.g., Butler et al. 2019; Ezcurra \& Butler 2018; Ezcurra et al. 2017;

289 Ezcurra et al. 2019; Maidment et al. 2020; Nesbitt et al. 2017a; Nesbitt et al. 2015; Pritchard et al. 2018;

290 Pritchard \& Nesbitt 2017; Pritchard \& Sues 2019; Scheyer et al. 2020a; Sengupta et al. 2017; Spiekman

291 2018; Spiekman et al. 2020a; Stocker et al. 2017), represent the two separate datasets that most

292 comprehensively addressed "protorosaur" relationships. The former focused specifically on

293 tanystropheid relationships. It found Protorosaurus as the sister taxon to all other archosauromorphs,

294 whereas Prolacerta formed the sister taxon to Archosauriformes. Tanystropheidae was recovered as a

295 monophyletic clade and consisted of Macrocnemus, Amotosaurus, Tanystropheus, Langobardisaurus,

296 Tanytrachelos, and the new Hayden Quarry material that was presented therein. The character list

297 consisted of 200 characters, including novel characters and characters derived from many previous

298 analyses (Benton 1985; Benton \& Allen 1997; Conrad 2008; DeBraga \& Rieppel 1997; Dilkes 1998;

299 Gauthier 1984; Gauthier et al. 1988a; Gauthier et al. 1988b; Hutchinson et al. 2012; Jalil 1997; Merck

300 1997; Modesto \& Sues 2004; Müller 2004; Nesbitt 2011; Rieppel 1994). Ezcurra (2016) presented a very

301 extensive analysis of early archosauromorph interrelationships that used 600 characters to analyze 96

302 taxa. Out of these characters, 96 were new. The remaining characters were compiled from the literature

303 (mainly Desojo et al. 2011; Dilkes 1998; Dilkes \& Arcucci 2012; Ezcurra et al. 2015a; Ezcurra et al. 2010;

304 Ezcurra et al. 2014; Gower \& Sennikov 1996; Gower \& Sennikov 1997; Jalil 1997; Nesbitt 2011; Nesbitt et

305 al. 2015; Parrish 1992; Pritchard et al. 2015; Senter 2004; Trotteyn \& Ezcurra 2014). Like Pritchard et al.

306 (2015), it found Protorosaurus to be the sister taxon to all other archosauromorphs. Prolacerta was

307 recovered as the sister taxon to Kadimakara australiensis, and the clade formed by these two taxa was

Peer] reviewing PDF | (2020:08:51657:1:1:NEW 28 Feb 2021) 
308 the sister clade to Archosauriformes + Tasmaniosaurus triassicus. Boreopricea was found as the sister

309 taxon to Prolacertidae + Tasmaniosaurus + Archosauriformes, whereas Jesairosaurus formed the sister

310 taxon to a monophyletic Tanystropheidae, composed of Macrocnemus, Amotosaurus, and

311 Tanystropheus.

312 Overview of "protorosaur" taxa

313 In the following, an overview is provided of taxa which have been attributed to "Protorosauria", 314 but which have not been included in the present analysis, since they are either represented by insufficient 315 material for inclusion or because it is now widely considered that they are not closely related to 316 Protorosaurus speneri, Prolacerta broomi, or Tanystropheidae.

Aenigmastropheus parringtoni Ezcurra, Scheyer \& Butler 2014. Aenigmastropheus parringtoni is known from one specimen, UMZC T836, from the Wuchiapingian (middle late Permian) of Tanzania. It comprises five cervical and dorsal vertebrae, the distal part of a right humerus, the proximal part of the right ulna, and several small fragments. The specimen was first described by Parrington (1956) and was considered to be insufficiently preserved for a confident taxonomic diagnosis. However, it was noted that its morphology contained both "primitive" diapsid traits as well as archosaurian characteristics. The specimen was recently revised and assigned to a new taxon, which was recovered as the sister taxon to Protorosaurus speneri in a cladistic analysis (Ezcurra et al. 2014). A later analysis found Aenigmastropheus parringtoni as the sister taxon to all other archosauromorphs (Ezcurra 2016).

Kadimakara australiensis Bartholomai 1979. Kadimakara australiensis is known from two partial skulls first described by Bartholomai (1979). The holotype is represented by the postorbital region, whereas the other specimen comprises a partial snout. Although both specimens do not have any shared preserved regions, they were attributed to the same taxon based on their similar size and shared close similarity to Prolacerta broomi. The validity of Kadimakara australiensis has been questioned and Borsuk-Białynicka \& Evans (2009b) and Evans \& Jones (2010) considered the specimens to be congeneric with Prolacerta broomi. Ezcurra (2016) corroborated the close affinity of Kadimakara australiensis to Prolacerta broomi, but only considered the holotype in the revised diagnosis of the taxon therein, since the lack of overlapping morphology precludes the direct comparison between the holotype and referred specimen. Ezcurra (2016) argued in favour of the validity of Kadimakara australiensis, pointing out a medial fossa on the posterior half of the parietals as a distinguishing feature between this species and Prolacerta broomi. 
338

339

340

341

342

343

344

345

346

347

348

349

350

351

352

353

354

355

356

357

358

359

360

361

362

363

364

365

366

367

368

erroneous interpretation of the morphology of the postorbital bar. Kadimakara australiensis comes from the lower beds of the upper part of the Arcadia Formation, central Queensland, Australia, which are of Induan (earliest Triassic) age.

Megacnemus grandis Huene 1954. Megacnemus grandis was described based on one isolated long bone exceeding $20 \mathrm{~cm}$ in length, which was identified as a femur (Huene 1954). Although the provenance of the specimen is unknown, Huene (1954) considered the specimen to most likely derive from the Gogolin Formation (Lower Muschelkalk) of southwest Poland, which is lower Anisian (Middle Triassic) in age, based on a similarity in preservation to fossils known from this formation. Skawiński et al. (2015) reexamined the specimen and corroborated its "protorosaurian" affinities. However, they also considered the possibility that the specimen represents a humerus rather than a femur, and therefore only identified the bone as a propodial. It has not been included in any phylogenetic analyses due to its extremely poorly known morphology.

Trachelosaurus fischeri Broili \& Fischer 1918. Trachelosaurus fischeri is represented by three associated slabs originating from the Thüringischer Chirotheriensandstein (Buntsandstein) of Bernburg (late Olenekian to early Anisian, late Early to early Middle Triassic), north of Halle, Germany (Schoch 2019). The remains preserved on these slabs comprise a premaxilla, two additional incomplete skull elements, associated gastralia, a likely coracoid, an ilium, a femur, and many, mostly disassociated, vertebrae and ribs, representing all sections of the vertebral column. The cervical ribs are remarkable in that they are short and bifurcated posteriorly. Many of the dorsal vertebrae bear conspicuously wide transverse processes. Alongside these elements referred to Trachelosaurus fischeri pedal or manual imprints and several fish scales are also preserved on the slabs. This material was first mentioned and partially figured by Huene (1902) when it was only partially prepared, and it was considered to represent a taxon closely related to Protorosaurus speneri. After further preparation, the species Trachelosaurus fischeri was erected and fully described based on this material by Broili \& Fischer (1918). Therein, it was concluded that the specimen showed similarities to Protorosaurus speneri in the shape of the cervical vertebrae, but also differed distinctly from this taxon in the number of cervical vertebrae, which was interpreted to be 20 or 21 , the height of the neural spines of the dorsal vertebrae, and the shape of the femur, which is relatively shorter and stockier compared to Protorosaurus speneri (Broili \& Fischer 1918). Trachelosaurus fischeri was assigned to Sauropterygia and was suggested to have been closely related to nothosaurs. Huene (1944) later revised the material of Trachelosaurus fischeri based on the detailed drawings presented by Broili \& Fischer (1918) and only identified 15 cervical vertebrae, nine of which were 
369

370

371

372

373

374

375

376

377

378

379

380

381

382

383

384

385

386

387

388

389

390

391

392

393

394

395

396

397

398

399

400

preserved in articulation. Four additional vertebrae, which were interpreted as cervical vertebrae by Broili \& Fischer (1918), were reinterpreted as caudal vertebrae. Huene (1944) disagreed with the high vertebral number of the cervical column suggested by Broili \& Fischer (1918) and concluded that more than one individual of Trachelosaurus fischeri was preserved within the material. Furthermore, several disassociated vertebrae that are considerably smaller than the rest of the preserved vertebrae, were considered to belong to an unidentified taxon other than Trachelosaurus fischeri. Considering the reduced cervical count of Trachelosaurus fischeri, Huene (1944) found that its morphology was in clear correspondence with that of a "protorosaur", with the notable deviation in the morphology of the cervical ribs. Trachelosaurus fischeri has been incorporated in several previous phylogenetic analyses. It formed a large polytomy at the base of Prolacertiformes in the analysis of Evans (1988). In one of the analyses presented by Benton \& Allen (1997) Trachelosaurus fischeri formed a polytomy with Tanystropheus meridensis (now considered a junior synonym of Tanystropheus longobardicus, see Spiekman \& Scheyer 2019) and a clade comprising Tanystropheus longobardicus and Tanytrachelos ahynis. However, Trachelosaurus fischeri was excluded from the final analysis in this study to increase tree resolution. Similarly, Trachelosaurus fischeri was part of a large polytomy within Prolacertiformes in one analysis and subsequently excluded from the final analysis to increase tree resolution in Jalil (1997). Trachelosaurus fischeri was also omitted from the analyses to optimize tree resolution by Rieppel et al. (2003). A revision of the holotype of Trachelosaurus fischeri, including detailed figures, is desirable and should carefully consider how many individuals and taxa are represented on the slabs.

Prolacertoides jimusarensis Young 1973. Prolacertoides jimusarensis represents the first described "protorosaur" from China. It is known from a single, poorly preserved skull (IVPP V3233) and was considered to be closely related to Prolacerta broomi in its initial description in Chinese (Young 1973). Ezcurra (2016) provided a more detailed osteological description of the holotype in English, but Prolacertoides jimusarensis was omitted from the final analysis therein, due to a lack of preserved, phylogenetically informative morphological characters. In analyses 1 and 2 of Ezcurra (2016), which included Prolacertoides and other poorly represented taxa, Prolacertoides jimusarensis was positioned in a large polytomy including most early archosauromorphs with Protorosaurus speneri as the sister taxon of that clade. Prolacertoides jimusarensis has previously been included in the phylogenetic analyses of Benton \& Allen (1997), Evans (198), Jalil (1997), and Rieppel et al. (2003). Notably, Benton \& Allen (1997) retrieved Prolacertoides jimusarensis as the sister taxon to Trilophosaurus buettneri and to thus fall outside of the traditional "Protorosauria". However, its exact phylogenetic affiliations were questioned by all authors due to a lack of morphological information for the taxon. 
401 Rhombopholis scutulata Owen 1842. Rhombopholis scutulata was originally described as an amphibian

402 (Owen 1842). It is represented by a single block that contains a number of postcranial bones, including 403 four vertebrae, several ribs, and five limb elements that belong to at least two individuals (Benton \& 404 Walker 1996). In a revision of the reptile material from the Keuper Sandstone Group of England (Anisian, 405 Middle Triassic), this specimen together with some other specimens were considered to be closely related 406 to Macrocnemus bassanii (Walker 1969). Benton \& Walker (1996) provided a redescription of 407 Rhombopholis scutulata, and identified it as a "prolacertiform" metataxon, meaning that no 408 autapomorphies could be identified to distinguish it from other "prolacertiforms" and that the different 409 specimens possibly belong to more than one taxon.

410 Sharovipteryx mirabilis Sharov 1971. A virtually complete but poorly preserved specimen with long and 411 gracile hindlimbs and an apparent skin membrane present between the legs was initially described as 412 Podopteryx mirabilis and interpreted as a gliding reptile (Sharov 1971). However, because the name 413 Podopteryx was already occupied by a genus of damselflies, the taxon was renamed Sharovipteryx 414 mirabilis by Cowen (1981). Sharovipteryx mirabilis was described in detail by Gans et al. (1987). Although 415 the phylogenetic position of Sharovipteryx mirabilis is exceedingly hard to assess due to the lack of visible 416 morphological details, it has been incorporated in "Protorosauria" by various authors (e.g., Ivakhnenko \& 417 Kurochkin 2008; Peters 2000; Unwin et al. 2000).

418 Cosesaurus aviceps Ellenberger \& De Villalta 1974. Cosesaurus aviceps is known from a single specimen, 419 which represents an impression of a complete skeleton. As such, the outline of the specimen is well420 preserved, but the detailed morphology of the taxon is very poorly known. The specimen was found at 421 the Montral-Alcover outcrop (Ladinian, Middle Triassic), Sierra de Prades, Tarragona Province, Spain. Due 422 to the lack of morphological information, the phylogenetic affinities of Cosesaurus aviceps are unclear. It 423 was initially thought to represent an ancestor to birds (Ellenberger 1977; Ellenberger 1978; Ellenberger \& 424 De Villalta 1974). However, the now widely accepted view that birds represent a derived clade of theropod 425 dinosaurs refutes this hypothesis. Cosesaurus aviceps was redescribed by Sanz \& López-Martínez (1984) 426 and considered to bear many similarities to various "protorosaurs". Cosesaurus aviceps has also been 427 found among "protorosaurs" in subsequent phylogenetic analyses (Benton \& Allen 1997; Evans 1988; Jalil 428 1997; Rieppel et al. 2003). In a reinterpretation of previous analyses by Peters (2000), which has been 429 widely criticized (e.g., Hone \& Benton 2007), it was concluded that Pterosauria are a derived lineage within 430 "Prolacertiformes". This was largely based on several morphological characters observed in Cosesaurus 431 aviceps, as well as the poorly known, gracile reptiles Sharovipteryx mirabilis and Longisquama insignis 
432 (Peters 2000). Although Cosesaurus aviceps might represent a "protorosaur", the lack of morphological 433 information does not allow this taxon to be reliably incorporated in phylogenetic analyses, and recent 434 phylogenetic investigations into archosauromorph or "protorosaurian" affinities did not consider this 435 taxon.

436 Vritramimosaurus dzerzhinskii Sennikov 2005. The holotype of Vritramimosaurus dzerzhinskii is a single 437 cervical vertebra, and referred material comprises another cervical vertebra, a caudal vertebra, and two 438 additional, fragmentary vertebrae. They were originally discovered in 1953 and 1954 by B.P. Vjuschkov. 439 Vritramimosaurus dzerzhinskii has been described as a "large, specialized prolacertilian" (Sennikov 2005). 440 The material originates from the Rassypnaya locality of the Petropavlovka Formation, Orenburg Region, 441 Russia, which is of uppermost Olenekian (Early Triassic) age. Its estimated overall body size is at least three 442 meters, making Vritramimosaurus dzerzhinskii one of the larger early archosauromorphs and considerably 443 larger than Prolacerta broomi, to which it is considered to be closely related (Sennikov 2005). However, 444 the limited and fragmentary material allows for only a very limited comparison to other taxa and the taxon 445 has therefore not been included in phylogenetic analyses.

446 Microcnemus efremovi Huene 1940. The referred material of Microcnemus efremovi consists of several 447 isolated elements, comprising vertebrae, long bones, a partial scapulacoracoid, a largely complete 448 ischium, and possibly a few skull elements including teeth (Huene 1940). These specimens were recovered 449 from Early Triassic sites (Benthosuchus assemblage zone, Olenekian) in Vologda Oblast, Russia, from which 450 the proterosuchid Chasmatosuchus rossicus has also been described (Gower \& Sennikov 2000; Huene 451 1940). The cervical vertebrae of Microcnemus efremovi are elongate and amphicoelous, with all vertebrae 452 having been described as thin-walled (Huene 1940). The partial scapulacoracoid exhibits a strongly 453 posteriorly curved scapular blade as is typical of tanystropheids (e.g., Macrocnemus bassanii, PIMUZ T 454 4355; Tanystropheus longobardicus, PIMUZ T 1277). Additionally, Huene (1940) considered a fragment of 455 a maxilla and a partial mandible including teeth to be possibly referrable to Microcnemus efremovi. The 456 teeth were described as robust with an acrodont implantation, which deviates strongly from all known 457 archosauromorphs. Huene (1940) considered the cervical vertebrae and scapulacoracoid to be most 458 similar to those of Macrocnemus and Tanystropheus, whereas the dorsal and caudal vertebrae, as well as 459 the tentatively included skull elements, were considered most similar to Araeoscelis. From the same 460 localities Huene (1940) additionally referred a partial cervical vertebra to ?Protorosauridae gen. indet. 461 that was considerably larger than the material of Microcnemus efremovi, and erected a new taxon, 462 "Chasmatosuchus parvus", based on another partial vertebra. The latter element was found to be 
463 morphologically indistinguishable from Microcnemus efremovi by Sennikov (1995) and was thus 464 reassigned to this species. Postcranial material that was initially identified as Microcnemus sp. (Garyainov 465 \& Rykov 1973; Rykov \& Ochev 1966) was later reassigned to the tanystropheid taxon Augustaburiania 466 vatagini by Sennikov (2011). Microcnemus efremovi has not been included in any phylogenetic analyses 467 but was suggested to be closely related to Macrocnemus bassanii by Sennikov (2011).

"Exilisuchus tubercularis" Ochev 1979. "Exilisuchus tubercularis" is known from a single partial ilium from the Sludkian Gorizont of the Orenburg Region, Russia (early Olenekian, Early Triassic; Gower \& Sennikov 2000). It has most commonly been interpreted as a possible proterosuchian, although its taxonomic assignment is uncertain due to the highly limited available morphological information (Ezcurra 2016;

472 Gower \& Sennikov 2000; Ochev 1979). Ezcurra (2016) briefly described and figured the holotype and 473 recovered "Exilisuchus tubercularis" within Tanystropheidae based on the presence of a dorsally rimmed caudifemoralis brevis muscle origin on the lateroventral surface of the postacetabular process. "Exilisuchus tubercularis" has been considered a nomen dubium by Gower (1994) and Ezcurra (2016).

Malerisaurus robinsonae Chatterjee 1980 and "Malerisaurus langstoni" Chatterjee 1986. Malerisaurus robinsonae is known from two individuals that are part of the stomach content of two specimens of the phytosaur Parasuchus hislopi from the Maleri Formation (late Carnian, early Late Triassic) of central India (Chatterjee 1980). Another specimen from the Tecovas Member, lower Dockum Formation of western Texas, US, (Carnian to early Norian, early Late Triassic) was recognized as representing a taxon that was very closely related to Malerisaurus robinsonae and assigned to the new species Malerisaurus langstoni (Chatterjee 1986). However, this holotype and only known specimen is actually composed of elements belonging to several diapsid taxa, most notably Trilophosaurus buettneri (Spielmann et al. 2006). Therefore, "Malerisaurus langstoni" is no longer considered a valid taxon. Furthermore, the validity of the Indian Malerisaurus robinsonae was questioned, as this taxon also showed distinct similarities to Trilophosaurus buettneri (Spielmann et al. 2006). Following the original interpretation by Chatterjee (1980; 1986) of Malerisaurus robinsonae as a "protorosaur" closely related to Protorosaurus speneri, it has been incorporated in phylogenetic analyses (Benton 1985; Benton \& Allen 1997; Evans 1988; Jalil 1997; Rieppel et al. 2003). Malerisaurus robinsonae was removed from the final analyses due to insufficient character preservation in Benton (1985) and Rieppel et al. (2003), whereas it was retrieved as part of a polytomy within Archosauromorpha by Evans (1988). Benton \& Allen (1997) included only "Malerisaurus langstoni" in the final analysis and found it as the sister taxon to all included "protorosaurs" 
494

495

496

497

498

499

500

501

502

503

504

505

506

507

508

509

510

511

512

513

514

515

516

517

518

519

520

521

522

523

524

both Malerisaurus species as a single OTU and found it to be the sister taxon to Jesairosaurus lehmani. Malerisaurus spp. have not been included in any of the recently published phylogenetic analyses of early archosauromorphs. Recently, Nesbitt et al. (2017b) identified both Malerisaurus species as separate from Trilophosaurus buettneri, and considered them to belong to Allokotosauria, more specifically as members of Azendohsauridae.

Malutinisuchus gratus Ochev 1986. Malutinisuchus gratus is a very poorly known taxon that has been considered a prolacertid. It is known from the Belyaevsky I, Bukobay Svita, Ladinian, Orenburg region, Russia (Ochev 1986; Tverdokhlebov et al. 2003). The known material comprises several fragmentary remains, including an elongated cervical vertebra, two partial limb bones, and likely pectoral girdle elements. Malutinisuchus gratus was incorporated into phylogenetic analyses by Jalil (1997) and Rieppel et al. (2003), but in both cases omitted from the final analysis due to a lack of morphological information. In one of the trees recovered by Jalil (1997), Malutinisuchus gratus formed a polytomy with all other taxa forming the clade "Prolacertiformes" therein.

Boreopricea funerea Tatarinov 1978. Boreopricea funerea is known from a nearly complete specimen and an anterior end of a snout, collected from a borehole, number 141, at 1112.3 meters deep at Kolguyev Island in the Barents Sea. This borehole is part of the Vetluzhian Series (Induan, earliest Triassic; Benton \& Allen 1997). The referred specimen is likely lost (Benton \& Allen 1997). Boreopricea funerea was originally considered to represent an intermediate form between Prolacerta broomi and Pricea longiceps (now considered a junior synonym of Prolacerta broomi), and Macrocnemus bassanii (Tatarinov 1978). The taxon was later redescribed in more detail by Benton \& Allen (1997), who commented on the poor state of the specimen and the absence of certain elements described by Tatarinov (1978) as a consequence of damage that the holotype had sustained after this description, such as the crushing of the skull and the displacement and in some cases disappearance of certain postcranial elements. Among these are the interclavicle and ossified sternum, which contained characters that were important in distinguishing Boreopricea funerea from other "protorosaurs". Furthermore, because these elements were removed and later placed back on the card on which the specimen is kept, the identification of the tarsal bones is difficult and ambiguous (Rieppel et al. 2003). Boreopricea funerea has been included in several phylogenetic analyses (Benton \& Allen 1997; Evans 1988; Ezcurra 2016; Jalil 1997; Rieppel et al. 2003) and an emended diagnosis was provided by Ezcurra (2016). Boreopricea funerea was found as the sister taxon to a clade comprising Macrocnemus, Cosesaurus, Tanystropheus, and Tanytrachelos within a monophyletic Protorosauria (Prolacertiformes therein) that also included Prolacerta (Evans 1988). In the 
525 phylogenetic analysis accompanying the redescription of the taxon, Boreopricea funerea represented the 526 sister taxon to Prolacerta (Benton \& Allen 1997). In the final tree of Jalil (1997) Boreopricea funerea was 527 recovered within a tanystropheid clade together with Cosesaurus, Tanystropheus, and Tanytrachelos. In 528 the various trees produced by Rieppel et al. (2003) the placement of Boreopricea funerea varied. In some 529 cases it was positioned as closely related to Protorosaurus and in others as being more closely related to 530 Prolacerta. Ezcurra (2016) recovered Boreopricea funerea as the sister taxon to the clade composed of 531 Prolacertidae, Tasmaniosaurus triassicus, and all Archosauriformes. Because of the badly damaged nature 532 of the holotype, certain previously observed cranial characters by Tatarinov (1978) and Benton \& Allen 533 (1997) could not be scored based on personal observation by Ezcurra (2016). Characters in which 534 Tatarinov (1978) and Benton \& Allen (1997) were in disagreement were scored as missing data by Ezcurra 535 (2016).

536 Eorasaurus olsoni Sennikov 1997. Eorasaurus olsoni, one of the very few known Permian 537 archosauromorphs, is known from several vertebrae. The taxon was originally considered to be most 538 closely related to Protorosaurus and was therefore placed within "Protorosauridae" (Sennikov 1997). 539 Ezcurra et al. (2014) provided additional observations and an emended diagnosis for Eorasaurus olsoni, 540 and it was retrieved as an archosauriform that formed a trichotomy with Euparkeria capensis and 541 Erythrosuchus africanus in the included phylogenetic analysis. Eorasaurus olsoni was also included by 542 Ezcurra (2016) and formed a massive polytomy at the base of Archosauriformes in the analyses 1 and 2 543 therein, but it was pruned from the final analysis due to insufficient phylogenetically informative 544 morphology being currently known for the taxon.

545 Hayden Quarry tanystropheid. Recently many postcranial elements with clearly tanystropheid affinities 546 were described, encompassing vertebrae, femora, and a calcaneum (Pritchard et al. 2015). Because the 547 material is represented by isolated elements, it is unclear whether they all belong to the same species, 548 and it was therefore not referred to any specific taxon. The calcaneum was shown to share apomorphies 549 with the calcanea of Tanytrachelos ahynis and this element was therefore assigned to this species. This 550 material was collected from the Hayden Quarry in western North America, which is of approximately 551 middle Norian age (Late Triassic; Irmis et al. 2011), making it among the youngest known material 552 referrable to Tanystropheidae. Although it was not concluded that the Hayden Quarry material represents 553 a single taxon, a hypothetical Hayden Quarry taxon was included in the phylogenetic analysis of Pritchard 554 et al. (2015), in which it was recovered as the sister taxon to the North American, Late Triassic 555 Tanytrachelos ahynis. Nevertheless, since the material represents only limited postcranial material, which 
556 furthermore cannot unequivocally be assigned to a single taxon, it is not considered for our phylogenetic 557 analysis.

558 Gwyneddosaurus erici Bock 1945. Gwyneddosaurus erici is known from a single specimen from the 559 Lockatong Formation near the town of Gwynedd, close to Philadelphia, Pennsylvania. The Lockatong 560 Formation is of late Carnian age (Late Triassic), and is approximately contemporaneous to the Cow Branch 561 Formation from which the better known Tanytrachelos ahynis is known (Colbert \& Olsen 2001). The only 562 known specimen was originally described by Bock (1945) and considered to be closely related to 563 Podokesaurus holyokensis, a poorly known early theropod dinosaur (Carrano \& Sampson 2004). The 564 holotype and only known specimen represents a disarticulated skeleton preserving several vertebrae, 565 ribs, parts of the pectoral girdle, and limb elements. Huene (1948) revised the specimen and identified it 566 as a "protorosaur" similar to Macrocnemus. Pritchard et al. (2015) suggested that this material might be 567 referrable to the same taxon as Tanytrachelos ahynis, although this hypothesis was credited to Olsen 568 (1979) therein. However, although he considered some tanystropheid material from the lower Lockatong 569 Formation to be referrable to Tanytrachelos, Olsen (1979) suggested that Gwyneddosaurus erici was quite 570 distantly related to Tanytrachelos ahynis.

571 Drepanosaurus unguicaudatus Pinna 1980. Drepanosaurus unguicaudatus was first descibed based on the 572 holotype, which consists of a largely complete, articulated skeleton, missing the skull and anterior cervical 573 vertebrae, and several juvenile specimens (Pinna 1980). This was followed by a more extensive description 574 (Pinna 1984), and these findings were later summarized in English (Pinna 1986). Renesto (1994c) revised 575 the morphology of Drepanosaurus unguicaudatus, especially regarding the highly specialized forelimbs, 576 and it was suggested that the juvenile specimens belong to a different species, Megalancosaurus 577 preonensis. This suggestion was reinforced by Renesto (2000), who considered the holotype as the only 578 known specimen of Drepanosaurus unguicaudatus, whilst a juvenile specimen (MCSNB 4783), previously 579 described by Renesto \& Paganoni (1995), was attributed to Drepanosaurus sp. A revised diagnosis and 580 overview of the provenance of drepanosaurid species was provided in Renesto et al. (2010). Pritchard et 581 al. (2016) described new remains from North America, which were assigned to Drepanosaurus sp. and 582 provided new insight into the unique configuration of the grasping forelimb of the taxon. Drepanosaurus 583 unguicaudatus is among the best-known drepanosaurids and has been included in several phylogenetic 584 analyses (Dilkes 1998; Evans 1988; Pritchard \& Nesbitt 2017; Pritchard et al. 2016; Renesto et al. 2010; 585 Senter 2004). However, Drepanosaurus unguicaudatus was omitted from the final analysis of Evans (1988) 586 due to a lack of observable phylogenetically informative characters. In the same analysis another 
587 drepanosaurid, Megalancosaurus preonensis, was recovered as the sister taxon to a large 588 "Prolacertiformes" clade. Benton \& Allen (1997) also included Megalancosaurus preonsensis as the only 589 drepanosaurid in their analysis, in which it was recovered as the sister taxon to Protorosaurus speneri. 590 Dilkes (1998) included both Drepanosaurus unguicaudatus and Megalancosaurus preonensis, which 591 formed the sister clade to Tanystropheidae in that analysis. Senter (2004) included five drepanosaurid 592 OTUs, which were found in a monophyletic clade that, together with the sister clade formed by 593 Coelurosauravus sp. and Longisquama insignis, were referred to Avicephala. This clade was recovered 594 outside Neodiapsida and thus, this was the first cladistic analysis to indicate that drepanosaurids were 595 quite distantly related to tanystropheids. Based on modifications of the data matrices of Dilkes (1998) and 596 Laurin (1991), as well as a newly formed character matrix, Renesto et al. (2010) concluded that the 597 drepanosaurids, including Drepanosaurus unguicaudatus, formed the sister clade to Tanystropheidae 598 within a monophyletic Protorosauria. Finally, data matrices employed by Pritchard et al. (2016) and 599 Pritchard \& Nesbitt (2017), which greatly enhanced both character and taxon sampling, recovered a 600 monophyletic Drepanosauromorpha including Drepanosaurus unguicaudatus outside Sauria. Due to their 601 highly derived morphology and because they likely represent a lineage outside Archosauromorpha, Drepanosaurus unguicaudatus and other drepanosauromorphs are not included in the present analysis. Vallesaurus cenensis Wild 1991. Vallesaurus cenensis is known from a single, well-preserved and complete specimen that was discovered in the Cene quarry, which represents the upper part of the Zorzino 605 Limestone (Revueltian, early-middle Norian, Late Triassic), in Lombardy, Italy (Renesto \& Binelli 2006). 606 Wild (1991) mentioned the specimen and assigned it to the genus Vallesaurus but did not formally 607 describe it. The specimen (Renesto 2000) and species (Pinna 1993) were subsequently referenced to, but 608 a formal description was only provided later by Renesto \& Binelli (2006). Vallesaurus cenensis has 609 additionally been compared to other drepanosaurids by Renesto et al. (2010). Therein, the new species 610 Vallesaurus zorzinensis was included in the genus. This species differs from Vallesaurus cenensis in having 611 an opposable hallux with two phalanges, of which the first one is straight. Vallesaurus cenensis has been 612 included in phylogenetic analyses (Pritchard \& Nesbitt 2017; Pritchard et al. 2016; Renesto et al. 2010; 613 Senter 2004).

614 Megalancosaurus preonensis Calzavara, Muscio \& Wild 1980. Megalancosaurus preonensis is known from 615 middle Norian Forni Dolostone of Friuli and Zorzino Limestone of Lombardy, Italy (Renesto et al. 2010).

616 The holotype of Megalancosaurus preonensis, which comprises a complete skull and cervical series, the 617 expanded neural spines of the anterior dorsal vertebrae, several fragments of dorsal ribs, and a right 
618 forelimb, was described by Calzavara et al. (1980) and interpreted as an arboreal archosaur. Feduccia \& 619 Wild (1993) and Feduccia (1996) suggested that Megalancosaurus preonensis was possibly a glider and 620 considered it to be closely related to birds, thus arguing that a lineage of Triassic non-dinosaurian621 archosauromorphs, rather than theropod dinosaurs, are the sister group to birds. An additional specimen 622 of Megalancosaurus preonensis was described, which provided new information on the postcranium of 623 the taxon (Renesto 1994a). Additionally, three specimens that were previously identified as juvenile 624 specimens of Drepanosaurus unguicaudatus (Pinna 1980) were re-assigned to the taxon as well (Renesto 625 1994a). The arboreal lifestyle suggested for Megalancosaurus preonensis was questioned by Padian \& 626 Chiappe (1998) and they instead considered an aquatic lifestyle for the taxon. The hypothesis that 627 drepanosaurids are the sister group to birds was refuted in a study that also assigned two additional 628 specimens to the species (Renesto 2000). The skull of Megalancosaurus preonensis was redescribed in 629 detail by Renesto \& Dalla Vecchia (2005). A second species, Megalancosaurus endennae, was erected and 630 two specimens that were previously identified as Megalancosaurus preonensis were re-assigned to this 631 species (Renesto et al. 2010). Megalancosaurus endennae mainly differs from Megalancosaurus 632 preonensis in the presence of an opposable hallux in the pes. Another specimen lacking the hindlimb, 633 MFSN 18443a, was reassigned to Megalancosaurus sp. A functional interpretation of the forelimbs of 634 Megalancosaurus spp. was provided by Castiello et al. (2016). Megalancosaurus preonensis has been 635 included in several phylogenetic analyses, the results of which are outlined above in the description of 636 Drepanosaurus unguicaudatus (Benton \& Allen 1997; Dilkes 1998; Evans 1988; Pritchard \& Nesbitt 2017; 637 Pritchard et al. 2016; Renesto et al. 2010; Senter 2004).

638 The following taxa are included as OTUs for the phylogenetic analysis:

Age. Late Missourian, late Pennsylvanian, Late Carboniferous.

641 Occurrence. Garnett Quarry, Rock Lake Member of the Stanton Formation, Kansas, USA (Reisz 1981; Reisz 642 et al. 1982).

643 Holotype. KUVP 1424, largely complete right hindlimb.

644 Referred specimens. The referred specimens are listed in Reisz (1981, p. 4-5).

645 Diagnosis. The emended diagnosis is provided by Reisz (1981). 
646 Remarks. Petrolacosaurus kansensis was first described based on a largely complete hindlimb (the 647 holotype KUVP 1424) and pelvis, and identified as a pelycosaur (Lane 1945). Additional postcranial 648 elements from the same locality were assigned to Podargosaurus hibbardi in the same study. Additional 649 specimens, including skull material, revealed that Podargosaurus was indistinguishable from 650 Petrolacosaurus kansensis and therefore its material was reassigned to the latter (Peabody 1952). The 651 systematic position of Petrolacosaurus kansensis was disputed, but an additional specimen preserving the 652 skull in more detail revealed it as an early diapsid reptile (Reisz 1977) and it has been described in detail 653 by Reisz (1981). Petrolacosaurus kansensis represents one of the best-known Carboniferous diapsids and 654 as such has been widely used as an outgroup or important early taxon in studies on saurian or diapsid 655 phylogeny (e.g., Dilkes 1998; Evans 1988; Ezcurra 2016; Ezcurra et al. 2014; Jalil 1997; Pritchard et al. 2015; 656 Simões et al. 2018). A recent phylogenetic hypothesis of early amniotes suggests that the diapsid temporal 657 configuration of Petrolacosaurus kansensis was likely independently acquired from that of neodiapsids, 658 including saurians (Ford \& Benson 2020).

659

Orovenator mayorum Reisz, Modesto \& Scott 2011

661

662 663

664

665

666

667

668

669

670

671

672

673

674

Age. Earliest Artinskian, early Permian (Cohen et al. 2013; Woodhead et al. 2010).

Occurrence. Claystone fissure fills in the Ordovician Arbuckle Limestone, Dolese Brothers Limestone Quarry, Richards Spur, Comanche County, Oklahoma, USA.

Holotype. OMNH 74606, a crushed partial skull missing several elements, including large parts of the skull roof and occipital region.

Referred specimen. OMNH 74607, a crushed partial skull preserving most of the skull roof, as well as an axis, two postaxial cervical vertebrae, and a single caudal vertebra.

Diagnosis. The most recent diagnosis was provided by Ford \& Benson (2018).

Remarks. Orovenator mayorum is an early Permian reptilian known from the Richards Spur locality, which represents a unique upland fissure fill deposit (Ford \& Benson 2020; MacDougall et al. 2017; Sullivan et al. 2000). Therefore, it was hypothesized that early reptilians from this locality were adapted to an upland environment, and that this in turn would explain their rare occurrence in the fossil record in the early and middle Permian (Reisz et al. 2011). Following the initial description and phylogenetic analysis including Orovenator mayorum by Reisz et al. (2011), in which it was recovered as the sister-taxon to all other 
675 known neodiapsids, the taxon was redescribed by Ford \& Benson (2018) based on $\mu C T$ scans. An extensive 676 recent phylogenetic analysis recovered Orovenator mayorum as a member of Varanopidae, a clade 677 historically considered to belong to Synapsida, but which was there found on the reptilian lineage outside 678 Neodiapsida (Ford \& Benson 2020). The cranial morphology suggests nocturnality and burrowing behavior 679 in Orovenator mayorum (Ford \& Benson 2018).

680

Age. Lopingian (roughly equivalent to the Dicynodon AZ of the Karoo Basin), late Permian (Smith et al. 683 2012).

684

685

686 687
Occurrence. Sakamena River Valley, Lower Sakamena Formation, southern Madagascar (Currie 1980).

Holotype. MNHN 1908-32-57, a skeleton preserved partially as imprints in two slabs. The posterior half of the skull and mandible is preserved. Of the postcranium, most of the dorsal vertebral series is preserved, as well as part of the forelimbs, the pelvis and sacral region, and hindlimbs.

Diagnosis. The most recent emended diagnosis is provided by Ezcurra (2016).

Remarks. Acerosodontosaurus piveteaui was first described by Currie (1980) and indicated to be closely related to Youngina capensis. A redescription of the only specimen of Acerosodontosaurus piveteaui revealed that the infratemporal bar is incomplete, in contrast to Youngina capensis (Bickelmann et al. 2009). An aquatic lifestyle has been suggested for Acerosodontosaurus piveteaui, which is supported by observed skeletal paedomorphosis of the carpal bones. Recent phylogenetic analyses recovered Acerosodontosaurus piveteaui as a diapsid that is closely related to Sauria (e.g., Bickelmann et al. 2009; Ezcurra et al. 2014; Ford \& Benson 2020; Pritchard \& Nesbitt 2017; Pritchard \& Sues 2019). One recent analysis addressing the relationships of stem-turtles recovered Acerosodontosaurus piveteaui in a clade with Claudiosaurus germaini within Pantestudines as the sister group to all other members of this clade (Li et al. 2018).

\section{Claudiosaurus germaini Carroll 1981}

Age. Lopingian (roughly equivalent to the Dicynodon AZ of the Karoo Basin), late Permian (Smith et al. 2012).

Peer) reviewing PDF | (2020:08:51657:1:1:NEW 28 Feb 2021) 
703 Occurrence. Lower Sakamena Formation near the village of Leoposa, southern Madagascar (Caldwell 704 1995; Carroll 1981).

705 Holotype. MNHN 1978-6-1, a largely complete skeleton, including a poorly preserved skull but missing the 706 posterior tail section.

707 Referred specimens. A list of referred specimens can be found in Carroll (1981; p. 337-338). Several 708 specimens are located in private collections. Additional undescribed specimens are housed in the SAM 709 (Simões et al. 2018).

710 Diagnosis. The diagnosis was provided by Carroll (1981).

711 Remarks. Claudiosaurus germaini is a non-saurian diapsid known from various specimens from the late

712 Permian of southern Madagascar. Its depositional environment, as well as its morphology, as indicated by 713 the enlarged hindlimbs and pedes, and skeletal paedomorphosis, suggest it had an aquatic lifestyle 714 (Caldwell 1995; Carroll 1981).

715

716

Youngina capensis Broom 1914

717 Age. Capitanian to Changhsingian, middle to late Permian (Rubidge et al. 2013; Smith \& Evans 1996)

718 Occurrence. Tropidostema, Cistecephalus, and Dicynodon AZs (Assemblage Zones), Balfour and Middelton 719 formations of the Beaufort Group, part of the Karoo Supergroup, South Africa (Broom 1914; Smith \& Evans 720 1996).

721 Holotype. AMNH 5561, a complete skull and mandible and a partial articulated vertebral column.

722 Referred specimens. The most inclusive hypodigm has been provided by Ezcurra (2016), who 723 supplemented a previous hypodigm of Gow (1975) with specimens found since then.

724 Diagnosis. The most recent emended diagnosis of Youngina capensis was provided by Ezcurra (2016).

725 Remarks. Youngina capensis is a middle to late Permian non-saurian neodiapsid with a generalized 726 morphology known from an array of well-preserved specimens. Its morphology has been investigated 727 frequently (e.g., Broom 1914; Broom 1922; Currie 1981; Evans 1987; Gardner et al. 2010; Goodrich 1942;

728 Gow 1975; Smith \& Evans 1996; Watson 1957). Youngina capensis represents an important taxon for 729 phylogenetic analyses that investigate early diapsid and saurian relationships (e.g., Ezcurra 2016; Ezcurra 
730

731

732

733

734

735

736

737

738

739

740

741

742

743

744

745

746

747

748

749

750

751

752

753

754

755

756

757

et al. 2014; Ford \& Benson 2020; Simões et al. 2018). Specimens that were previously assigned to other taxa, Youngoides romeri (Olson \& Broom 1937), Youngoides minor (Broom \& Robinson 1948), Youngopsis kitchingi (Broom 1937), Youngopsis rubidgei (Broom \& Robinson 1948), and Acanthotoposaurus bremneri (Evans \& Van Den Heever 1987), have all been shown to be conspecific with Youngina capensis (Evans 1987; Gow 1975; Reisz et al. 2000).

\section{Gephyrosaurus bridensis Evans 1980}

Age. Hettangian, and possibly Sinemurian, Early Jurassic (Whiteside et al. 2016).

Occurrence. Pontalun quarry of the St. Brides fauna, southern Wales (Evans \& Kermack 1994; Whiteside \& Duffin 2017; Whiteside et al. 2016).

Holotype. UCL T.1503, a right dentary.

Referred specimens. The material of Gephyrosaurus bridensis comprises an extensive amount of isolated remains (over 1,000 specimens following Evans 1980). No complete list of referred specimens is currently available in the literature.

Diagnosis. The diagnosis was provided by Evans (1980, p. 204-205)

Remarks. Gephyrosaurus bridensis is exclusively known from extensive isolated remains that have been assigned to a single taxon based on the complementary articulation surfaces between the various elements, as well as their morphological similarity and relative size. Gephyrosaurus bridensis has been described in detail in one study addressing the skull (Evans 1980), and another addressing the postcranium (Evans 1981). Although known from younger, Early Jurassic, deposits, Gephyrosaurus bridensis is considered the sister taxon to the clade encompassing the Triassic rhynchocephalians Planocephalosaurus robinsonae, Clevosaurus spp., and other more crownward rhynchocephalians (e.g., Hsiou et al. 2015; Scheyer et al. 2020a; Simões et al. 2018). An additional species of this genus, Gephyrosaurus evansae, was recently described from the Rhaetian (Late Triassic) 'Microlestes' quarry at Holwell near Bristol, UK (Whiteside \& Duffin 2017).

Planocephalosaurus robinsonae Fraser 1982

Age. Early Rhaetian, Late Triassic (Whiteside et al. 2016).

Peer) reviewing PDF | (2020:08:51657:1:1:NEW 28 Feb 2021) 
758 Occurrence. Late Triassic fissure fills of Cromhall and Tytherington quarries, Bristol and Gloucestershire,

759 UK (Fraser 1982; Whiteside \& Marshall 2008).

760 Holotype. AUP No. 11061, an isolated left maxilla.

761 Referred specimens. As for Gephyrosaurus bridensis, Planocephalosaurus robinsonae is represented by a 762 large amount of isolated elements (at least 750 specimens from Cromhall quarry according to Fraser 763 1982), and no complete list of referred specimens is currently available in the literature.

764 Diagnosis. The diagnosis of Planocephalosaurus robinsonae is provided by Fraser (1982, p. 710).

765 Remarks. Like Gephyrosaurus bridensis, Planocephalosaurus robinsonae is known from the Late Triassic 766 to Early Jurassic fissure fills of southwestern England and southern Wales. However, whereas 767 Gephyrosaurus bridensis is known from an Early Jurassic Welsh locality, Planocephalosaurus robinsonae is 768 known from Late Triassic English localities. Its material is also composed of a large amount of three769 dimensionally preserved, isolated remains that can be assigned to a single taxon based on their relative 770 connectivity, morphological similarity, and size (although Simões et al. 2018 considered the assignment 771 of postcranial elements to this taxon only tentative). The skull was described by Fraser (1982), and the 772 postcranium has subsequently been described by Fraser \& Walkden (1984). Planocephalosaurus 773 robinsonae represents one of the best-known early rhynchocephalians and bears several plesiomorphic 774 features compared to Clevosaurus spp. and other more derived rhynhocephalians. Specimens of 775 Planocephalosaurus have also been identified from the Ruthin quarry, southern Wales, but were not 776 assigned to the species level (Whiteside et al. 2016). Small tooth bearing fragments from the lower 777 Tecovas Formation, Chinle Group (late Carnian) in Texas, USA, were assigned to a new species, 778 Planocephalosaurus lucasi (Heckert 2004).

Protorosaurus speneri Meyer 1832

Age. Traditionally Tatarian, although conodont data points towards a more specific Wuchiapingian age, late Permian (Ezcurra et al. 2014; Legler \& Schneider 2008).

Occurrence. The Middridge and Quarrington quarries near Durham, Marl Slate, England (Evans \& King 1993) and various localities of the Kupferschiefer Formation of central Germany (all localities are listed in Table 1 of Gottmann-Quesada \& Sander 2009). 
786 Lectotype. Since no formal holotype had previously been assigned, NHMW 194314, known as the

787 Swedenborg specimen, was assigned the lectotype by Gottmann-Quesada \& Sander (2009).

788 Referred specimens. Table 1 of Gottmann-Quesada \& Sander (2009) listed 28 specimens that were 789 included in that study. More specimens that can tentatively be assigned to the species are known, which 790 are distributed among various institutions and private collections across Europe, and a complete 791 hypodigm is missing. Most specimens consist of postcranial material, whereas skull material is 792 comparatively rare and only known from five different specimens: RCSHC/Fossil Reptiles 308, WMsN P 793 47361, TWCMS S1348(.1 and .2), IGWuG 463016, and NMK S 180. Only NMK S 180 represents a complete 794 and well-preserved skull.

795 Diagnosis. Ezcurra (2016) provided the most recent diagnosis for the species.

796 Remarks. Protorosaurus speneri currently represents by far the best-known Permian archosauromorph.

797 The first specimen to be discovered, RCSHC/Fossil Reptiles 308 or the Spener specimen, was described by 798 Spener (1710) and interpreted as a fossil of a Nile crocodile (Crocodylus niloticus). Protorosaurus speneri 799 was erected and described in detail based on additional material (Meyer 1830; Meyer 1832; Meyer 1856). 800 Other specimens were described more recently (e.g., Evans \& King 1993; Fichter 1995; Haubold \& 801 Schaumberg 1985), and the species was extensively revised by Gottmann-Quesada \& Sander (2009). Most 802 of the approximately 40 known specimens derive from the Kupferschiefer Formation of Germany, 803 whereas two come from the contemporary Marl Slate of England (Evans \& King 1993). Most recent 804 phylogenetic analyses recovered Protorosaurus speneri as one of the earliest diverging archosauromorphs 805 (e.g., Ezcurra 2016; Pritchard et al. 2015; for an alternative placement of Protorosaurus speneri and 806 tanystropheids outside Archosauromorpha, see Simões et al. 2018).

807

808 Czatkowiella harae Borsuk-Białynicka \& Evans 2009

809 Age. Earliest late Olenekian, Early Triassic (Shishkin \& Sulej 2009).

810 Occurrence. Czatkowice 1, a fissure or cave infill of the Czatkowice quarry near Kraków, Poland.

811 Holotype. ZPAL R.V/100, an isolated, nearly complete right maxilla bearing teeth. 
812 Referred specimens. A large number of isolated cranial and postcranial elements that could confidently

813 be distinguished from other tetrapod remains of the Czatkowice 1 locality. A large number of these bones 814 are presented and described by Borsuk-Białynicka \& Evans (2009b).

815 Diagnosis. The diagnosis was provided by Borsuk-Białynicka \& Evans (2009b).

816 Remarks. The material now referred to Czatkowiella harae was originally discovered in 1978 at Czatkowice

817 1. It is represented by many isolated and fragmented specimens, which were found among similar remains 818 belonging to other small diapsids, such as the euparkeriid Osmolskina czatkowicensis (Borsuk-Bialynicka 819 \& Evans 2003; Borsuk-Białynicka \& Evans 2009a; Borsuk-Białynicka \& Sennikov 2009), the 820 lepidosauromorph Sophineta cracoviensis (Evans \& Borsuk-Białynicka 2009), the kuehneosaurid Pamelina 821 polonica (Evans 2009), and three distinct procolophonids (Borsuk-Białynicka \& Lubka 2009). Apart from 822 the most diagnostic elements, bones were assigned to Czatkowiella harae largely based on size and fitting 823 individual elements together. The most distinguishing feature of Czatkowiella harae is the presence of 824 three-headed anterior dorsal ribs. It has only been considered phylogenetically by Borsuk-Białynicka \& 825 Evans (2009b), who recovered Czatkowiella harae as the sister taxon to Protorosaurus speneri. The 826 disarticulated and fragmented remains of Czatkowiella harae were found in a bonebed comprising a 827 diverse fauna and it is possible that multiple taxa are represented among its referred material (Ezcurra et 828 al. 2014), making this taxon somewhat problematic for inclusion in phylogenetic analyses. Here, we follow 829 the identification by Borsuk-Białynicka \& Evans (2009b) in all but the most tentatively assigned bones (e.g., 830 the squamosal) and as for all other taxa scored, the specimen(s) referred to for the scoring of each 831 character are provided in the Supplementary Material. This allows subsequent workers to critically 832 evaluate scorings, as well as exclude certain specimens that further investigation might find belong to a 833 different taxon. Because the inclusion of potentially composite taxa can negatively influence the accuracy 834 of phylogenetic analyses, Czatkowiella harae is omitted from the analyses 2 and 4 here.

Tanystropheus longobardicus Bassani 1886

837 Age. Latest Anisian-Ladinian, Middle Triassic (Spiekman et al. 2020a; Spiekman \& Scheyer 2019; Stockar 838 2010).

839 Occurrence. Besano Formation and the Cassina beds, Meride Limestone, of Monte San Giorgio, 840 Switzerland and Italy. 
841 Neotype. The holotype specimen was destroyed in Milan during World War II (Nosotti 2007; Spiekman \& 842 Scheyer 2019; Wild 1973). A neotype was established by Wild (1973): PIMUZ T 2791 - An almost complete 843 and largely articulated, bituminous specimen, lacking the posterior half of the tail.

844 Referred specimens. PIMUZ T 2779, PIMUZ T 2781, PIMUZ T 2795, PIMUZ T 2485, PIMUZ T 2482, PIMUZ 845 T 2484, PIMUZ T 3901, PIMUZ T 1277, MSNM BES SC 265, and MSNM BES SC 1018.

846 Diagnosis. The most recent diagnosis for the taxon was provided by Spiekman et al. (2020a, Methods S1).

847 Remarks. Tanystropheus longobardicus was first described based on a single, partially articulated 848 specimen from the Besano Formation of Monte San Giorgio. It was interpreted as a pterosaur and 849 assigned to Tribelesodon longobardicus, with the generic name referring to the tricuspid teeth that were 850 present in the jaws (Arthaber 1922; Bassani 1886; Nopcsa 1923). This specimen has unfortunately been 851 lost, but it has been figured in Arthaber (1922). The discovery of additional specimens from the Besano 852 Formation revealed that the elements that were interpreted as elongated phalanges represented 853 elongated cervical vertebrae that were similar to those known from the Upper Muschelkalk of the 854 Germanic Basin, which had been assigned to Tanystropheus conspicuus (Peyer 1930; Peyer 1931). 855 Therefore, the species was re-assigned to Tanystropheus longobardicus. Wild (1973) described the species 856 in detail and assigned PIMUZ T 2791 as the neotype. Additional specimens were described in Wild (1980a), 857 including a specimen from the slightly younger Meride Limestone, which was assigned to the separate 858 species Tanystropheus meridensis. However, this specimen, as well as an additional specimen that was 859 found from the Meride Limestone (Renesto 2005), were shown to be morphologically indistinguishable 860 from the specimens from the Besano Formation, and therefore Tanystropheus meridensis was considered 861 a junior synonym of Tanystropheus longobardicus (Nosotti 2007; Spiekman \& Scheyer 2019). A small-sized 862 Tanystropheus skeleton lacking the skull from the Zhuganpo Formation of China was identified as 863 Tanystropheus sp. and could represent the only known occurrence of Tanystropheus longobardicus from 864 China, indicating a Tethys-wide distribution of the species (Li 2007). However, since no diagnostic cranial 865 material is known for this specimen, it cannot be assigned to the species level and therefore the presence 866 of Tanystropheus longobardicus in China is currently unclear (Spiekman et al. 2020a, Methods S1; 867 Spiekman \& Scheyer 2019). Nosotti (2007) described in detail specimens from the Italian side of the

868 Besano Formation. Recently, a combined morphological and palaeohistological study revealed that the 869 small-sized specimens of Tanystropheus from Monte San Giorgio, which bear distinct tricuspid marginal 870 teeth, are skeletally mature (Spiekman et al. 2020a). This indicates that the small-sized specimens 871 represent a separate species from the large-sized specimens, and the latter were re-assigned to the new 
872 species Tanystropheus hydroides. Tanystropheus longobardicus was therefore a relatively small-sized

873 Tanystropheus species, likely not exceeding 2 metres in total length, that fed on small prey, including soft874 shelled invertebrates.

875

876 Tanystropheus hydroides Spiekman, Neenan, Fraser, Fernandez, Rieppel, Nosotti \& Scheyer 2020

877 Age. Latest Anisian-earliest Ladinian, Middle Triassic (Spiekman et al. 2020a; Stockar 2010).

878 Occurrence. Besano Formation of Monte San Giorgio, Switzerland and Italy.

879 Holotype. PIMUZ T 2790, a compressed skull and anterior eight cervical vertebrae in semi-articulation.

880 Referred specimens. MSNM BES 351, MSNM V 3663, PIMUZ T 1270, PIMUZ T 1307, PIMUZ T 2480, PIMUZ

881 T 2483, PIMUZ T 2497, PIMUZ T 2787, PIMUZ T 2788, PIMUZ T 2793, PIMUZ T 2818, PIMUZ T 2819, PIMUZ

882 T 183, PIMUZ T 2817, SNSB-BSPG 1953 XV 2. For additional information, see Spiekman et al. (2020b).

Diagnosis. The diagnosis for Tanystropheus hydroides has been provided in Spiekman et al. (2020a, p. 884 3890).

Remarks. Specimens of Tanystropheus hydroides were previously considered as the adult morphotype of Tanystropheus longobardicus, but it was recently shown that they represent a separate, large-sized species (Spiekman et al. 2020a). Specimens of Tanystropheus hydroides were described as Tanystropheus longobardicus in Peyer (1931), Kuhn-Schnyder (1947; 1959), and Wild (1973). A Tanystropheus specimen, GMPKU P 1527, has been described from China that attained a size similar to Tanystropheus hydroides (Rieppel et al. 2010). Although the postcranial skeleton of GMPKU P 1527 cannot be distinguished from Tanystropheus hydroides, the absence of a skull has not allowed the specimen to be assigned to this species with certainty, and it is therefore currently considered as Tanystropheus cf. T. hydroides (Spiekman et al. 2020b). As such, it has been included as a separate OTU in the present analysis. However, it is clear that the genus had a Tethys-wide distribution (Spiekman \& Scheyer 2019). Tanystropheus hydroides has recently been interpreted as an aquatic ambush predator that employed its long-neck and a snapping bite to catch its prey, which consisted of fish and cephalopods (Spiekman et al. 2020a; Spiekman et al. 2020b). hydroides was provided by Spiekman et al. (2020b). 
900

901

902

903

904

905

906

907

908

909

910

911

912

913

914

915

916

917

918

919

920

921

922

923

924

925

926

927

GMPKU P 1527

Age. Ladinian, Middle Triassic (Sun et al. 2016).

Occurrence. The upper part of the Zhuganpo Formation of Nimaigu near Xingyi City, Wusha District, Guizhou Province, southwestern China.

Remarks. A large-sized Tanystropheus skeleton from China that is largely complete but lacks the skull, anterior segment of the neck, the posterior end of the tail, and most of the pedes, was identified as Tanystropheus cf. T. longobardicus (Rieppel et al. 2010). Recently, it was shown that the large-sized specimens of Tanystropheus from Monte San Giorgio represent a separate species from the small-sized specimens and they were assigned to Tanystropheus hydroides (Spiekman et al. 2020a). Therefore, the assignment of GMPKU P 1527 has consequently been altered to Tanystropheus cf. T. hydroides (Spiekman et al. 2020b). The recent distinction between Tanystropheus longobardicus and Tanystropheus hydroides has indicated that the skull appears to be considerably more variable than the postcranium between species within this genus. Therefore, since GMPKU P 1527 lacks any cranial material and because it is known from the eastern side of the Tethys Ocean, whereas the referred specimens of Tanystropheus hydroides derive from its western margin, this specimen cannot be unequivocally assigned to the species, even though its preserved postcranial skeleton was considered to be virtually morphologically indistinguishable from the known postcranium of Tanystropheus hydroides (Rieppel et al. 2010; Tanystropheus hydroides is referred to as the adult type of Tanystropheus longobardicus therein). GMPKU P 1527 was incorporated as a separate OTU here to test its phylogenetic position relative to the known Tanystropheus species.

\section{Tanystropheus "conspicuus" Meyer 1855}

Age. Late Anisian to Ladinian, Middle Triassic (Menning \& Hendrich 2016; Spiekman \& Scheyer 2019).

Occurrence. Various localities of the Upper Muschelkalk and Lettenkeuper of Central Europe. An overview of all known localities can be found in Supplementary Table 1 of Spiekman \& Scheyer (2019).

Lectotype. U-MO BT 740, an isolated, three-dimensionally preserved cervical vertebra.

Referred specimens. The referred specimens of Tanystropheus "conspicuus" are listed in Supplementary Table 1 of Spiekman \& Scheyer (2019).

Peer) reviewing PDF | (2020:08:51657:1:1:NEW 28 Feb 2021) 
928 Remarks. Several elongate bones from the Upper Muschelkalk of Bayreuth, Germany, were identified as 929 reptilian vertebrae and assigned to Tanystropheus "conspicuus" by Meyer (1855). These bones had 930 previously also been described by Count Georg zu Münster, who had interpreted these elements as limb 931 bones of a saurian reptile, which he had named "Macroscelosaurus". However, since this work has been 932 lost and this generic name has fallen into disuse (nomen oblitum), the generic name Tanystropheus has 933 received precedence (Melville 1981; Wild 1973, p. 148). Following the description of the semi-articulated 934 specimens of Tanystropheus longobardicus (Peyer 1930; Peyer 1931), Huene (1931) considered material 935 previously identified as "Thecodontosaurus latespinatus", "Thecodontosaurus primus", and 936 "Procerosaurus cruralis" from the Upper Muschelkalk of Europe to very likely belong to Tanystropheus 937 "conspicuus". Wild (1973) provided a systematic palaeontology section in which these taxa were 938 synonymized with Tanystropheus conspicuus. Fragmentary and isolated remains of Tanystropheus 939 "conspicuus" are known from the Upper Muschelkalk and Lettenkeuper throughout Central Europe (late 940 Anisian to Ladinian; Menning \& Hendrich 2016). This material comprises isolated cervical, dorsal, sacral, 941 and caudal vertebrae, two femora, and an ischium. Peyer (1931) refrained from providing a detailed 942 comparison of Tanystropheus longobardicus with Tanystropheus "conspicuus" and "Tanystropheus 943 antiquus" from the Germanic Basin. Wild (1973) distinguished Tanystropheus "conspicuus" from 944 Tanystropheus longobardicus on the basis of comparatively wider rib attachment sites and a concavity on 945 the anterior end of the neural spine of the cervical vertebrae. Although he considered these minor 946 differences to be insufficient for a species definition, the distinction between the two taxa was maintained 947 in expectation of additional specimens that would allow for a more complete comparison. A recent 948 revision of Tanystropheus spp. revealed that no distinct morphological differences could be identified 949 between Tanystropheus "conspicuus", Tanystropheus hydroides (therein the large morphotype of 950 Tanystropheus longobardicus), and Tanystropheus "haasi" (Spiekman \& Scheyer 2019). However, since 951 the hypodigms of both Tanystropheus "conspicuus" and Tanystropheus "haasi" are insufficient for a 952 detailed comparison and both are only known from fragmentary and isolated postcranial elements, these 953 taxa were considered as nomina dubia.

954

"Tanystropheus antiquus" Huene 1905

956 Age. Latest Olenekian to middle Anisian, latest Early Triassic to Middle Triassic (Menning \& Hendrich 2016; 957 Spiekman \& Scheyer 2019). 
958 Occurrence. Lower Muschelkalk of Silesia, Poland (Gogolin Formation), Germany (Schaumkalk Formation), 959 and the Netherlands (Vossenveld Formation) (see also Supplementary Table 1 of Spiekman \& Scheyer 960 2019).

961 Syntype. SMNS 16687, SMNS 10110, MGUWr 3872s, MGUWr 3888s, MGUWr 3895s, MGUWr 3902s and 962 some uncatalogued MGUWr specimens, all consisting of isolated cervical vertebrae. Wild (1973) assigned 963 SMNS 10110 as the lectotype.

964 Referred specimens. All specimens assigned to "Tanystropheus (c.f.) antiquus" are listed in Supplementary 965 Table 1 of Spiekman \& Scheyer (2019).

966 Diagnosis. Recent diagnoses were provided for this taxon by Sennikov (2011) for "Protanystropheus 967 antiquus" and Fraser \& Rieppel (2006) for "Tanystropheus antiquus".

968 Remarks. Following the description of the syntype of "Tanystropheus antiquus" from the Lower 969 Muschelkalk of Gogolin and Krapkowice, Silesia, Poland (Huene 1905), other isolated Tanystropheus-like 970 remains from the Lower Muschelkalk were attributed to the species (e.g., Huene 1931; Kuhn 1971; 971 Schmidt 1928; Schmidt 1938; Spiekman et al. 2019; Wild \& Oosterink 1984). Ortlam (1966) referred 972 material of the uppermost Buntsandstein (Anisian) to Tanystropheus longobardicus and Macrocnemus 973 bassanii, but this material was later assigned to "Tanystropheus antiquus" by Wild (1980b). The 974 Buntsandstein precedes the Muschelkalk and in contrast to the latter represents largely fluvial sediments 975 (Feist-Burkhardt et al. 2008), and Wild (1980b) concluded that the discovery of "Tanystropheus antiquus" 976 from the Buntsandstein indicated that at least the juvenile individuals of this species had a terrestrial 977 lifestyle. Both Wild (1987) and Evans (1988) later suggested that "Tanystropheus antiquus" might belong 978 to a separate genus, based on the large morphological discrepancy between this taxon and other 979 Tanystropheus species. Fraser \& Rieppel (2006) revised the Buntsandstein specimens and concluded that 980 it represented a separate taxon from the Lower Muschelkalk specimens of "Tanystropheus antiquus" and 981 assigned it to the new species Amotosaurus rotfeldensis. Despite a lack of diagnostic characters in the 982 material, Fraser \& Rieppel (2006) tentatively maintained the assignment of the Lower Muschelkalk 983 specimens to "Tanystropheus antiquus". Sennikov (2011) compared "Tanystropheus antiquus" to 984 Augustaburiania vatagini and concluded that the former was sufficiently distinct from Tanystropheus spp. 985 to assign it to a new genus, resulting in the combination "Protanystropheus antiquus".

986 The relative length of the cervical vertebrae might indeed indicate that "Tanystropheus antiquus" is more 987 closely related to Augustaburiania vatagini or Amotosaurus rotfeldensis than to other Tanystropheus 
988 species. However, the taxonomic status of "Tanystropheus antiquus" is currently unclear since many 989 specimens of the syntype material (Huene 1902; Huene 1905) were long considered to have been lost 990 (Fraser \& Rieppel 2006; Sennikov 2011; Wild 1973; Wild 1980b). However, these specimens have recently 991 resurfaced and were briefly discussed by Skawiński et al. (2017). Any taxonomic evaluation of this taxon 992 would first require a detailed revision of this type material to assess whether subsequently referred 993 specimens of "Tanystropheus antiquus" from other localities represent the same species (Spiekman et al. 994 2019; Spiekman \& Scheyer 2019). Such a revision is currently underway (Szczygielski, personal commun. 995 2019), and therefore the taxonomic status of "Tanystropheus antiquus" is not addressed here. However, 996 we include a preliminary "Tanystropheus antiquus" OTU in analyses 1 and 3 based on the strong 997 morphological similarity of the tanystropheid cervical vertebrae from the Lower Muschelkalk of Central 998 Europe. Our scoring of this OTU is based on two complete cervical vertebrae, SMNS 16687 and Coll. 999 Oosterink A638. The former specimen comes from the Lower Muschelkalk of Krapkowice, Poland, and 1000 constitutes part of the syntype of "Tanystropheus antiquus", and the latter derives from the Lower Muschelkalk of Winterswijk, the Netherlands (Spiekman et al. 2019; Wild \& Oosterink 1984).

1002

1003

Sclerostropheus fossai Wild 1980

1004

Age. Late Norian, Late Triassic (Rigo et al. 2009; Tackett \& Tintori 2019).

1005

Occurrence. N-slope of Canto Alto, near Poscante in Val Brembana, Bergamo Province, Italy (Wild 1980a).

1006

Holotype. MCSNB 4035, a partial, articulated cervical column.

1007

Diagnosis. The most recent diagnosis was provided by Spiekman \& Scheyer (2019).

1008

Remarks. Sclerostropheus fossai is known from a single specimen, which constitutes a partial, semi1009 articulated cervical column, and was previously considered within the genus Tanystropheus (Wild 1980a). However, the morphology of the cervical vertebrae and ribs differs distinctly from that of other Tanystropheus species, as was briefly indicated by Renesto (2005), and it was recently assigned to the new genus Sclerostropheus (Spiekman \& Scheyer 2019). After Langobardisaurus pandolfii, Sclerostropheus 1013 fossai represents a second tanystropheid taxon known from the Norian of northern Italy.

1014 
1016

1017

1018

1019

1020

1021

1022

1023

1024

1025

1026

1027

1028

1029

1030

1031

1032

1033

1034

1035

1036

1037

1038

1039

1040

1041

1042

1043

Age. latest Anisian-Ladinian, Middle Triassic.

Occurrence. Besano Formation and Meride Limestone of Monte San Giorgio, Switzerland and Italy (Jaquier et al. 2017; Peyer 1937; Renesto \& Avanzini 2002; Rieppel 1989; Stockar 2010).

Holotype. MSNM 14624, a cast of MSNM specimen Besano I, a poorly preserved specimen that was destroyed in Milan during WWII (Fraser \& Furrer 2013).

Referred specimens. Rieppel (1989, p. 374) provided a referred specimen list for the Macrocnemus bassanii material housed at PIMUZ. Among those specimens, the specimen listed there as A 111/208 is now catalogued as PIMUZ T 4822. Additionally, two specimens of Macrocnemus bassanii are housed in the MSNM: MSNM BES SC 111; a complete and fully articulated juvenile including skin remains; MSNM V 457, a disarticulated adult specimen, in which a number of skull and jaw bones are preserved, as well as several cervical, dorsal, and caudal vertebrae, gastralia, ribs, and both pelvic girdles and both hindlimbs, excluding the feet.

Diagnosis. The most recent diagnosis was provided by Jaquier et al. (2017).

Remarks. Macrocnemus bassanii is the type species of the genus and is known from the Middle Triassic of Switzerland and Italy. It was first described by Nopcsa (1930) based on the poorly preserved holotype. This specimen was lost during World War II, but a cast has been preserved. The taxon was described in more detail following the discovery of multiple well-preserved specimens (Peyer 1937). Further details of the skull were provided by Kuhn-Schnyder (1962) and Rieppel \& Gronowski (1981). The postcranium and its functional considerations were discussed by Rieppel (1989), which indicated that Macrocnemus bassanii was facultatively bipedal. An excellently preserved juvenile specimen preserving soft tissue was described by Premru (1991) and Renesto \& Avanzini (2002). The skull and atlas-axis complex of Macrocnemus bassanii were recently redescribed in detail with the use of a synchrotron microtomography scan, revealing several previously obscured anatomical regions, such as the braincase (Miedema et al. 2020). Macrocnemus bassanii is currently firmly established as a tanystropheid (e.g., Ezcurra 2016; Pritchard et al. 2015).

Macrocnemus fuyuanensis Li, Zhao \& Wang 2007

Age. Late Anisian-Ladinian, Middle Triassic (Stockar 2010; Sun et al. 2016).

PeerJ reviewing PDF | (2020:08:51657:1:1:NEW 28 Feb 2021) 
1044 Occurrence. Besano Formation of Monte San Giorgio, Switzerland and Zhuganpo Formation of Huabi, Yun1045 nan Province, China (Jaquier et al. 2017; Jiang et al. 2011; Li et al. 2007; Scheyer et al. 2020b).

1046 Holotype. IVPP V15001, a mostly complete and largely articulated skeleton missing most of the skull.

1047 Referred specimens. GMPKU-P-3001, almost complete and fully articulated specimen, missing most of the 1048 tail; PIMUZ T 1559, virtually complete and disarticulated specimen, missing parts of the skull, almost the 1049 complete tail, and the hindlimbs.

1050 Diagnosis. The most recent diagnosis for the species was provided by Scheyer et al. (2020b).

1051 Remarks. The holotype specimen of Macrocnemus fuyuanensis was first described by Li et al. (2007) and 1052 distinguished from Macrocnemus bassanii based on the relative proportions of the limbs as well as the 1053 number of dorsal vertebrae. Jiang et al. (2011) described another specimen of Macrocnemus fuyuanensis 1054 with a completely preserved skull, GMPKU-P-3001, and concluded that it differed from Macrocnemus 1055 bassanii in several cranial characters. A specimen from the upper Besano Formation of Switzerland, PIMUZ T 1559, was described by Jaquier et al. (2017). This specimen was more similar in limb proportions to Macrocnemus fuyuanensis than to Macrocnemus bassanii, and also differed from the latter in the morphology of the interclavicle, and it was therefore identified as Macrocnemus aff. M. fuyuanensis. Furthermore, the cranial morphology of Macrocnemus fuyuanensis specimen GMPKU-P-3001 was revised in this study, indicating that it did not substantially differ from that of Macrocnemus bassanii. The holotype IVPP V15001 was redescribed, which revealed new anatomical details for the taxon, particularly with regards to the palate and pectoral girdle (Scheyer et al. 2020b). The morphology of the interclavicle of the holotype was in correspondence with that of the Swiss specimen PIMUZ T 1559, and distinctly differed from that of specimens assigned to Macrocnemus bassanii. Therefore, PIMUZ T 1559 was reassigned to Macrocnemus fuyuanensis and the species thus occurred on both the eastern and western margins of the Tethys Ocean.

1067

Macrocnemus obristi Fraser \& Furrer 2013

Age. Early Ladinian, Middle Triassic.

1070 Occurrence. Prosanto Formation of Ducanfurgga near Davos, canton Graubünden, Switzerland (Fraser \& 1071 Furrer 2013). 
1072 Holotype. PIMUZ A/III 1467 (housed in the Bündner Naturmuseum, Chur, Switzerland), an articulated 1073 partial skeleton, which consists of the posterior dorsal vertebrae, pelvic girdle and hindlimbs, and most of 1074 the tail.

1075 Referred specimens. PIMUZ A/III 722, a right pes preserved in dorsal view.

1076 Diagnosis. The diagnosis was provided by Fraser \& Furrer (2013, p. 200)

1077 Remarks. Macrocnemus obristi is known from two specimens from the Prosanto Formation (Fraser \& 1078 Furrer 2013). It differs from Macrocnemus bassanii and Macrocnemus fuyuanensis based on the length 1079 proportions of the femur and tibia (Fraser \& Furrer 2013; Jaquier et al. 2017). Due to its recent description 1080 and only partially known morphology, Macrocnemus obristi has previously only been included in the 1081 phylogenetic analysis of Ezcurra \& Butler (2018), in which it was scored for the purpose of a disparity 1082 analysis. Therein, Macrocnemus obristi was recovered within a monophyletic Macronemus clade as the 1083 sister taxon to Macrocnemus fuyuanensis.

1084

1085

1086

1087

1088

1089

1090

1091

1092

1093

1094

1095

1096

1097

1098

1099
Tanytrachelos ahynis Olsen 1979

Age. Late Carnian, early Late Triassic.

Occurrence. Virginia Solite Quarry B, Upper member of the Cow Branch Formation, part of the Dan River Group (Newark Supergroup), USA (Casey et al. 2007; Liutkus-Pierce et al. 2014; Olsen 1979).

Holotype. YPM 7496, a largely complete, articulated specimen.

Referred specimens. A hypodigm was listed by Olsen (1979, p. 4-5, and note on p. 13). Most specimens are housed in the VMNH.

Diagnosis. The diagnosis for Tanytrachelos ahynis was provided by Olsen (1979)

Remarks. Tanytrachelos ahynis was described by Olsen (1979) and is known from hundreds of specimens from Solite Quarry B in Virginia (Casey et al. 2007). However, many detailed morphological features are unknown for Tanytrachelos ahynis, due to the poor preservation of the specimens. Recently, the authors of the current study subjected a relatively well-preserved specimen (NMS G.2017.11.1) to synchrotron radiation micro-computed tomography. This revealed the inner anatomy of the cervical vertebrae in some detail, highlighting that as in Tanystropheus spp. and Macrocnemus bassanii the neural canal passes through the vertebral centrum in Tanytrachelos ahynis. However, most morphological details could not 
1100 be observed due to the poor preservation of the specimen, which is likely attributable to diagenetic factors 1101 (Liutkus-Pierce et al. 2014). The Solite Quarry B is represented by lacustrine deposits (Fraser et al. 1996) 1102 and Tanytrachelos ahynis is considered to have had an aquatic lifestyle (Casey et al. 2007; Olsen 1979). As 1103 in Tanystropheus longobardicus and Tanystropheus hydroides, paired heterotopic bones parallel to the 1104 anterior caudal vertebrae occur in approximately half of the articulated specimens preserving this region, 1105 which indicates that the presence of these elements is likely related to sexual dimorphism.

AMNH FARB 7206

Age. Carnian, early Late Triassic (Colbert \& Olsen 2001)

Occurrence. Lockatong or Stockton Formation of Hudson County, New Jersey, USA.

Remarks. Small reptilian specimens have been recovered from the Lockatong Formation of New Jersey, which are approximately contemporaneous to the Cow Branch Formation in Virginia from which Tanytrachelos ahynis is known. Although it could not be excluded that some of these specimens might represent Tanytrachelos ahynis, not enough diagnostic features were preserved to positively identify any of these specimens to this taxon (Olsen 1979). However, one of the best-preserved specimens, AMNH FARB 7206, was recently referred to Tanytrachelos ahynis by Pritchard et al. (2015). This specimen was here scored as a separate OTU to test this hypothesis. Additionally, Pritchard et al. (2015) referred a single calcaneum from the middle Norian Hayden Quarry locality of New Mexico to Tanytrachelos ahynis based on the striking similarities in morphology between this element and the calcaneum of AMNH FARB 7206. Since it is currently uncertain whether the latter can be referred to Tanytrachelos ahynis, we also consider the assignment of the Hayden Quarry calcaneum to Tanytrachelos ahynis as equivocal. Therefore, only specimens from the Solite Quarry B can currently be confidently assigned to Tanytrachelos ahynis. 2006; Ortlam 1966). 
1127 Holotype. SMNS 50830, a largely disarticulated skeleton, including an articulated cervical series, maxilla,

1128 parabasisphenoid, scapulacoracoids and pelvic girdles, and scattered dorsal vertebrae.

1129 Referred specimens. Many specimens housed in the SMNS, some of which are unprepared or 1130 unaccessioned, including: SMNS 54783 a and b, a slab and counterslab preserving two mostly 1131 disarticulated skeletons, including a poorly preserved skull roof, cervical vertebrae, an articulated dorsal 1132 vertebral series, three articulated hindlimbs including pedes, and a partial forelimb including manus; 1133 SMNS 50691, three slabs, preserving a partial pes, a coracoid and maxilla, and a partial skull in ventral 1134 view, ilium, and dorsal vertebrae, respectively; SMNS 54784a and b, a slab and counterslab preserving the 1135 palatal region of a skull and a partial cervical series; SMNS 54810, disarticulated skeletons, including both 1136 cranial and extensive postcranial remains; SMNS 90600, posterior part of the vertebral column, including 1137 sacral and anterior caudal vertebrae; SMNS 90601, articulated maxilla and jugal; SMNS 90540, two skulls 1138 in palatal view; SMNS unnumbered (1), partial mandible and cervical vertebrae and ribs; SMNS 1139 unnumbered (2), disarticulated cranial elements and a partial cervical series; SMNS unnumbered (3), skull 1140 in palatal view three anterior cervical vertebrae; SMNS unnumbered (4), sacral region.

1141 Diagnosis. The diagnosis of Amotosaurus rotfeldensis was provided by Fraser \& Rieppel (2006, p. 867).

1142 Remarks. Several specimens of associated skeletons from the Buntsandstein of Baden-Württemberg were 1143 assigned to Macrocnemus bassanii and Tanystropheus longobardicus by Ortlam (1966). However, Wild 1144 (1980b) considered this material to represent juvenile specimens of "Tanystropheus antiquus", which is 1145 known from several isolated remains, mostly cervical vertebrae, from the Lower Muschelkalk of the 1146 Germanic Basin (Spiekman \& Scheyer 2019). Fraser \& Rieppel (2006) re-examined the specimens from the 1147 Buntsandstein and assigned it to the new taxon Amotosaurus rotfeldensis. The genus was named in 1148 honour of R. Wild. Ezcurra (2016) and Pritchard et al. (2015) incorporated Amotosaurus rotfeldensis in 1149 their phylogenetic analyses and provided several new morphological observations for the taxon.

Age. Latest Olenekian, latest Early Triasssic.

1153 Occurrence. Donskaya Luka locality, right slope of the Don River valley, Lipovskaya Formation, Ilovlyanskii 1154 District, Volgograd Region, Russia (Sennikov 2011). 
1156 Referred specimens. The referred specimens are listed in Sennikov (2011, p. 98).

1157 Diagnosis. The diagnosis was provided by Sennikov (2011).

1158 Remarks. Augustaburiania vatagini is known from the latest Olenekian (Early Triassic) of Donskaya Luka 1159 of the Don River valley, Russia, and thus represents one of the earliest known tanystropheids together 1160 with likely tanystropheid material from the Sanga do Cabral Formation (Induan-early Olenekian) of Brazil 1161 (De Oliveira et al. 2018; De Oliveira et al. 2020). Like other tanystropheids, such as "Tanystropheus 1162 antiquus" and Tanystropheus "conspicuus", Augustaburiania vatagini is solely known from isolated 1163 postcranial remains largely represented by cervical vertebrae. The relative length of the mid-cervical vertebrae of Augustaburiania vatagini is longer than that of "Tanystropheus antiquus" and Amotosaurus rotfeldensis, but shorter than that of other Tanystropheus species. Furthermore, the cervical vertebrae of Augustaburiania vatagini can be distinguished by a distinct concave margin of the centrum of the cervical vertebrae, although the expression of this character in the referred material is subject to much intraspecific variation. The number of cervical vertebrae of Augustaburiania vatagini was considered to be eight or nine by Sennikov (2011) but cannot be unambiguously established, since no articulated cervical vertebrae have been preserved.

Age. Julian, early Carnian, early Late Triassic.

Occurrence. Predil Limestone near Prasnig Brook, Tarvisio, Udine Province, Italy (Dalla Vecchia 2020). Holotype. MFSN 27532, a partial skeleton comprising the thoracic part of the vertebral column, a single partial cervical vertebra, sacral vertebrae, part of the pelvic girdle and left femur, and a purported tooth.

Diagnosis. The diagnosis was provided by Dalla Vecchia (2020).

Remarks. Raibliania calligarisi was recently described from a single specimen from the early Carnian of northern Italy. It is closely related to Tanystropheus longobardicus and is slightly younger than the known occurrence of this species (Spiekman et al. 2020a). Raibliania calligarisi is distinguished from Tanystropheus longobardicus based on differences in the shape of the neural spines of the dorsal vertebrae, the pleurapophyses of the posterior dorsal vertebrae, the iliac blade, the anterior portion of the pubis, and a single, disarticulated tooth (Dalla Vecchia 2020). The identification of the single, isolated 
1184 tooth is somewhat equivocal, as it is located far from where the head would have been preserved.

1185 Raibliania calligarisi is here considered in a phylogenetic context for the first time. Another specimen 1186 discovered in the vicinity of the holotype of Raibliania calligarisi, MFSN 13228, consisting of three 1187 articulated caudal vertebrae, represents the only other described tetrapod remains from the locality. This 1188 specimen has not been referred to Raibliania calligarisi due to the lack of overlapping morphology 1189 between it and the holotype (Dalla Vecchia 2020). Although the relative size of the vertebrae corresponds 1190 to that of the holotype of Raibliania calligarisi, the morphology of the neural spine differs distinctly from that of Tanystropheus longobardicus, to which Raibliania calligarisi is closely related.

Prolacerta broomi Parrington 1935

Age. Induan, Early Triassic.

Occurrence. Middle Beaufort beds, Lystrosaurus AZ, Katberg Formation, South Africa; Transanctartic Mountains, Fremouw Formation, Antarctica (Groenewald \& Kitching 1995; Peecook et al. 2019). Holotype. UMZC 2003.40 - A partial skull and mandible.

Referred specimens. The referred specimens are listed in Spiekman (2018, p. 4-5).

Diagnosis. The latest diagnosis of Prolacerta broomi was provided by Spiekman (2018).

1200

1201

1202

1203

1204

1205

1206

1207

1208

1209

1210

1211

1212

Remarks. Prolacerta broomi was first described by Parrington (1935) based on a crushed partial skull found in the Katberg Formation, Lystrosaurus Zone, near Harrismith, South Africa. Prolacerta broomi has played an important role in discussions on the evolutionary origin of modern reptile groups and has been considered both as an ancestral lepidosaur (e.g., Camp 1945; Parrington 1935) and archosaur (e.g., Romer 1956). Prolacerta broomi was first identified as a "protorosaur" by Camp (1945). Following the discovery of more specimens, the complete morphology of Prolacerta broomi, including the postcranium, was described (Gow 1975). This revealed that Pricea longiceps Broom \& Robinson 1948 represented a junior synonym of Prolacerta broomi. The braincase of Prolacerta broomi was described by Evans (1986). Based on new specimens as well as a reappraisal of previously described South African material, Modesto \& Sues (2004) provided a redescription of the skull of Prolacerta broomi. Specimens of Prolacerta broomi have also been described from Antarctica, consisting of several smaller, likely juvenile specimens, and a single, large-sized specimen, which is slightly larger than the specimens known from South Africa (Colbert 1987; Spiekman 2018). Although previously considered a member of the "protorosaurs", recent phylogenetic 
1213 analyses indicate that Prolacerta broomi is more closely related to Archosauriformes than Protorosaurus 1214 speneri and tanystropheids (e.g., Dilkes 1998; Ezcurra 2016; Modesto \& Sues 2004; Pritchard et al. 2015; 1215 Rieppel et al. 2003). Prolacerta broomi has been used widely as an outgroup in phylogenetic analyses 1216 focused on Archosauriformes and early crown-archosaurs (e.g., Butler et al. 2015; Desojo et al. 2011; 1217 Dilkes \& Sues 2009; Nesbitt 2011; Sookias 2016).

Ozimek volans Dzik \& Sulej 2016

Age. Late Carnian or early Norian, Late Triassic (Dzik \& Sulej 2016; Szulc et al. 2017).

1221

Occurrence. Grabowa Formation (Silesian Keuper) of Krasiejów, Upper Silesia, Poland.

Holotype. ZPAL AbIII/2512, partial skeleton missing the skull.

Referred specimens. A complete hypodigm can be found in Dzik \& Sulej (2016).

Diagnosis. The diagnosis was provided by Dzik \& Sulej (2016).

1225

Remarks. Ozimek volans was recently described based on several partial and disarticulated skeletons (Dzik \& Sulej 2016). An elongate vertebra now referred to this taxon was previously linked to either pterosaurs or Tanystropheus due to its extreme elongation (Dzik \& Sulej 2007). It is considered a close relative of the gliding reptile Sharovipteryx mirabilis and was possibly also a glider, although a comparison is limited due to the poorly known morphology of Sharovipteryx mirabilis. The morphology of Ozimek volans is highly derived and differs distinctly from other "protorosaurs" in the relative length and gracile construction of the limb bones and the configuration of the pectoral girdle, which includes an enlarged coracoid and possibly an ossified sternum. Formally assigned to the family Sharovipterygidae, Ozimek volans was considered a "protorosaur" based on the presence of elongate cervical vertebrae, the posterior curvature of the scapula, and the procoelous articulation surfaces of the cervical vertebrae (which occur in Tanytrachelos ahynis and Langobardisaurus pandolfii among tanystropheids, but which is widespread among some other diapsids, e.g., drepanosaurids; Dzik \& Sulej 2016). Unfortunately, the skull morphology of Ozimek volans is only partially known and identification of many cranial bones is uncertain due to their disassociation and peculiar morphology. Ozimek volans has been included in the phylogenetic analysis of Pritchard \& Sues (2019), in which it was recovered within Tanystropheidae as the sister taxon to a clade comprising Langobardisaurus pandolfii and Tanytrachelos ahynis. 
1242 Elessaurus gondwanoccidens De Oliveira, Pinheiro, Da Rosa, Dias da Silva \& Kerber 2020

1243 Age. Induan-Olenekian, Early Triassic (De Oliveira et al. 2020; Dias-da-Silva et al. 2017).

1244 Occurrence. Bica São Tomé, Sanga do Cabral Formation, São Francisco de Assis, Rio Grande do Sul, 1245 southern Brazil.

1246 Holotype. UFSM 11471, a left hindlimb, partial pelvis, a single sacral vertebra and three caudal vertebrae.

1247 Diagnosis. The diagnosis was provided by De Oliveira et al. (2020).

1248 Remarks. Elessaurus gondwanoccidens is known from a single hind limb, partial pelvis, and a few sacral 1249 and caudal vertebrae, and has been identified as the sister taxon to Tanystropheidae (De Oliveira et al. 1250 2020). In addition to Elessaurus gondwanoccidens, several isolated cervical vertebrae with a typical 1251 tanystropheid morphology (De Oliveira et al. 2018), as well as the non-archosauriform crocopod 1252 Teyujagua paradoxa (Pinheiro et al. 2019), are also known from the Sanga do Cabral Formation. However, 1253 due to the limited morphological information currently available for Elessaurus gondwanoccidens, its 1254 phylogenetic placement is somewhat uncertain.

Jesairosaurus lehmani Jalil 1997

1257 Age. Late Olenekian-early Anisian, late Early Triassic to early Middle Triassic (Jalil 1999).

1258 Occurrence. Site 5003 of Busson, at the base of the Zarzaitine Formation, Algeria.

1259 Holotype. ZAR 06, a nearly complete skull and mandible, the neural arches of the five posteriormost 1260 cervical vertebrae, the complete left and partial right pectoral girdle, and the proximal end of the left 1261 humerus.

1262 Referred specimens. The hypodigm was provided by Jalil (1997) and Ezcurra (2016).

1263 Diagnosis. The most recent diagnosis was provided by Ezcurra (2016).

1264 Remarks. The specimens now assigned to Jesairosaurus lehmani were originally interpreted as 1265 procolophonid remains (Lehman 1971). However, detailed observation was hampered by a hard hematite 1266 layer that covered the specimens. Additional preparation revealed the diapsid affinity of the material (Jalil 
1267 1990), and it was later described in detail and assigned to Jesairosaurus lehmani (Jalil 1997). More recently

1268 a morphological redescription of Jesairosaurus lehmani was provided by Ezcurra (2016). Jalil (1997) 1269 identified Jesairosaurus lehmani as a "protorosaur" and in a phylogenetic analysis found it to be the sister 1270 taxon to "Malerisaurus langstoni", whereas the clade they formed was recovered as the sister group to a

1271 tanystropheid clade that included Boreopricea funerea and Cosesaurus aviceps. In a re-analysis of this 1272 matrix by Rieppel et al. (2003), Jesairosaurus lehmani formed a polytomy with other "protorosaurs" 1273 (including drepanosaurids) and a clade composed of Prolacerta broomi and the archosauriforms 1274 Proterosuchus and Euparkeria. In the recent analysis by Ezcurra (2016), Jesairosaurus lehmani was 1275 recovered as the sister taxon to Tanystropheidae. The unstable position of Jesairosaurus lehmani might 1276 be related to the poorly resolved relationships of former "protorosaurs" and the difficulties to confidently 1277 assess morphological details for this taxon because of the preservation of the specimens in a hematite 1278 layer, which has severely hampered their preparation (Jalil 1997).

1279

1280

Langobardisaurus pandolfii Renesto 1994

1281

Age. Alaunian/Revueltian, middle Norian, Late Triassic.

1282

Occurrence. The uppermost section of the Zorzino Limestone Cene quarry, Lombardy, Italy (Renesto 1994b), lower member of the Forni Dolostone of Friuli, Italy (Renesto \& Dalla Vecchia 2000), and the Seefeld Formation, near Innsbruck, Austria (Saller et al. 2013).

Holotype. MCSNB 2883 , an articulated partial skeleton, missing both forelimbs completely, as well as parts of the skull, feet, and tail.

1287

1288

1289

1290

1291

1292

1293

1294 1295

Referred specimens. MCSNB 4860, complete and articulated juvenile preserved in ventral view, with the skull covered by the neck and trunk; MFSN 1921, a virtually complete and articulated adult specimen, including a well-preserved skull. Only the posterior part of the tail and part of the left forelimb are missing; MFSN 26829, a partial articulated adult specimen, preserving a nearly complete right hindlimb, a partial left hindlimb, and some poorly visible parts of the vertebral column and possibly the pelvic girdle; P 10121, a nearly complete impression of an articulated adult, only missing part of the tail, with some fragments of the limb bones and teeth preserved.

Diagnosis. The latest emended diagnosis for Langobardisaurus pandolfii was provided by Saller et al. (2013). 
1296 Remarks. Renesto (1994b) was the first to describe the genus Langobardisaurus based on two specimens 1297 originally found in 1974, which were assigned to Langobardisaurus pandolfii and considered to be closely 1298 related to known tanystropheids, specifically Macrocnemus bassanii. It was interpreted as a terrestrial 1299 insectivore based on the presence of tricuspid teeth, which among other archosauromorphs only occur in 1300 Tanystropheus longobardicus. Bizzarini \& Muscio (1995) proposed a new species, Langobardisaurus rossii, 1301 based on a new but poorly preserved specimen from the Forni Dolostone of Friuli, Italy. This specimen 1302 was later considered as a probable rhynchocephalian lepidosauromorph, mainly inferred from its body 1303 proportions, particularly the relative size of the skull and cervical and trunk regions (Renesto \& Dalla Vecchia 2007). However, the poor preservation of this specimen prevents an unequivocal taxonomic determination. Another species, Langobardisaurus? tonelloi, was tentatively ascribed to the genus based on a complete specimen (MFSN 1921) by Muscio (1996). The species was considered to differ from Langobardisaurus pandolfii in its phalangeal formula and dentition. However, Renesto \& Dalla Vecchia (2000) could not find any differences between the phalangeal formula in these two taxa and considered the minor differences in dentition to be attributable to ontogenetic variation, and thus considered Langobardisaurus tonelloi to likely represent a junior synonym of Langobardisaurus pandolfii, which was later corroborated by Saller et al. (2013). MFSN 1921 allowed the first detailed description of the skull of Langobardisaurus pandolfii and revealed a unique dentition among archosauromorphs, consisting of an edentulous premaxilla and anterior margin of the maxilla, followed by tricuspid teeth more posteriorly on the maxilla and dentary, and terminating in a very large, molar-like crushing tooth on both the maxilla and dentary. Renesto \& Dalla Vecchia (2000) hypothesized that Langobardisaurus pandolfii used this highly specialized dentition to feed on large insects, crustaceans, and small scaly fishes. Renesto et al. (2002) described another specimen, MFSN 26829, and considered facultative bipedal locomotion for Langobardisaurus pandolfii, which has also been proposed for Macrocnemus bassanii (Rieppel 1989). P 10121, a poorly preserved specimen of Langobardisaurus pandolfii, consists of the impression, as well as bone fragments, of a nearly complete skeleton. This specimen was found in the Seefeld Formation of Austria, extending the biogeographic range of the taxon outside Italy (Saller et al. 2013). 
1325 Occurrence. Member II of the Guanling Formation of Xinmin close to Panxian, and Luoping, Guizhou

1326 Province, China (Li 2003; Liu et al. 2017; Rieppel et al. 2008).

1327 Holotype. IVPP V13767, an almost complete skull and the three anteriormost cervical vertebrae and 1328 associated ribs.

1329 Referred specimens. ZMNH M8752, an undescribed specimen of which the pelvic morphology was briefly 1330 mentioned in comparison to that of Fuyuansaurus by Fraser et al. (2013); LPV 30280, a partial, articulated 1331 skeleton including some disarticulated skull bones, most of the cervical column, parts of the thorax, 1332 hindlimbs, and anterior part of the tail. Within the thorax some elements belonging to an embryo are 1333 preserved, which indicates that this specimen was pregnant when it died (Liu et al. 2017); IVPP V13898, a 1334 relatively complete skeleton including a skull preserved in ventral view, a complete cervical series, and 1335 parts of the thorax including an articulated fore and hindlimb (Rieppel et al. 2008).

1336 Diagnosis. The most recent diagnosis of Dinocephalosaurus orientalis was provided by Rieppel et al. 1337 (2008).

1338 Remarks. Dinocephalosaurus orientalis was first described based exclusively on the holotype, which only 1339 preserves the skull and three anteriormost cervical vertebrae (Li 2003). The discovery of a specimen 1340 preserving much of the postcranial skeleton subsequently revealed a striking convergence between 1341 Dinocephalosaurus orientalis and Tanystropheus spp. (Li et al. 2004). Dinocephalosaurus orientalis shares 1342 the extreme elongation of the neck with Tanystropheus spp., but achieved this elongation through 1343 different means, since its neck iscomposed of at least 33 vertebrae that are comparatively short, whereas 1344 that of Tanystropheus hydroides and Tanystropheus longobardicus is composed of 13 hyperelongate cervical vertebrae (Li et al. 2017b; Rieppel et al. 2010; Rieppel et al. 2008). Additionally, the postcranial morphology of Dinocephalosaurus orientalis shows clear adaptations to a fully aquatic lifestyle, most notably in the paddle-like limbs. The unique morphology of Dinocephalosaurus orientalis has led to various hypotheses regarding its lifestyle and feeding method. Li et al. (2004) tentatively suggested suction feeding for Dinocephalosaurus orientalis, which was refuted by Peters (2005) and Demes \& Krause (2005). The former argued that Dinocephalosaurus orientalis was a benthic ambush predator and a very poor swimmer. This suggestion was in turn repudiated by LaBarbera \& Rieppel (2005). A general anatomical description of Dinocephalosaurus orientalis was provided by Rieppel et al. (2008). In this study Dinocephalosaurus orientalis was incorporated into the combined dataset provided by Rieppel et al. 1354 (2003) for a phylogenetic analysis, which found a polytomy formed by Jesairosaurus lehmani, 
1355

1356

1357

1358

1359

1360

1361

1362

1363

1364

1365

1366

1367

1368

1369

1370

1371

1372

1373

1374

1375

1376

1377

1378

1379

1380

1381

1382

Dinocephalosaurus orientalis, drepanosaurids, and tanystropheids. Liu et al. (2017) described a new Dinocephalosaurus specimen, which preserves articulated remains of a much smaller Dinocephalosaurus specimen within the thorax of the adult, indicating the first example of vivipary in an archosauromorph reptile. This study also provided an updated version of the phylogenetic analysis of Rieppel et al. (2008), in which they recovered Dinocephalosaurus orientalis as the sister taxon to Tanystropheidae. Another embryo bearing close similarities to Dinocephalosaurus orientalis was described by Li et al. (2017b). It represents a separate taxon, since it differs distinctly from Dinocephalosaurus orientalis in its relative limb proportions and in having 24 rather than at least 33 cervical vertebrae. However, it has not been assigned to a separate species due to the very early ontogenetic stage of the specimen and it was instead referred to as a "dinocephalosaur", indicating the presence of multiple closely related Dinocephalosaurus-like taxa.

\section{Fuyuansaurus acutirostris Fraser, Rieppel \& Li 2013}

Age. Ladinian, Middle Triassic (Sun et al. 2016).

Occurrence. Zhunganpo Formation of Guizhou Province, China (Fraser et al. 2013).

Holotype. IVPP V17983, a partial skeleton preserving a skull, cervical vertebral column, a few dorsal vertebrae, and a partial pectoral and pelvic girdle.

Diagnosis. The diagnosis of Fuyuansaurus acutirostris was provided by Fraser et al. (2013).

Remarks. Fuyuansaurus acutirostris is a small archosauromorph with a long neck and an elongate rostrum, known from a single, possibly juvenile, specimen. It has been interpreted as an aquatic taxon and bears typical tanystropheid features in the presence of a long neck composed of elongated cervical vertebrae and corresponding ribs, and a scapular blade that is semi-lunar in shape (Fraser et al. 2013). However, Fuyuansaurus acutirostris differs from other tanystropheids in its elongate and tapered rostrum and a pelvic girdle that lacks a thyroid fenestra between the pubis and ischium. Fuyuansaurus acutirostris has previously only been included in the phylogenetic analysis of Ezcurra \& Butler (2018), in which it was scored for the purpose of a disparity analysis. Therein it was recovered in a large polytomy within Tanystropheidae.

Pectodens zhenyuensis Li, Fraser, Rieppel, Zhao \& Wang 2017

PeerJ reviewing PDF | (2020:08:51657:1:1:NEW 28 Feb 2021) 
1383

1384

1385

1386

1387

1388

1389

1390

1391

1392

1393

1394

1395

1396

1397

1398

1399

1400

1401

1402

1403

1404

1405

1406

1407

1408

1409

Age. Anisian, Middle Triassic (Sun et al. 2016).

Occurrence. Member II of the Guanling Formation, Luoping, Yunnan Province, China Li et al. (2017a).

Holotype. IVPP V18578, a nearly complete and articulated skeleton including the skull.

Diagnosis. The diagnosis was provided by Li et al. (2017a).

Remarks. Pectodens zhenyuensis is a small, highly gracile archosauromorph with an elongate neck, tail, and limbs. It bears certain characteristics typical of "protorosaurs", most notably in having a long neck with elongate cervical vertebrae and ribs. Li et al. (2017a) addressed the similarities of Pectodens zhenyuensis to "Protorosauria" but considered its inclusion in this group only tentative since it lacks several diagnostic features, such as the presence of a hooked fifth metatarsal and a thyroid fenestra between the pubis and ischium, and because it differed from other "protorosaurs" in the shape of its skull and marginal dentition. Most carpal bones are missing in the only known specimen, even though the manus is fully articulated, possibly because these bones had not yet ossified due to the early ontogenetic stage of the specimen. The phylogenetic position of Pectodens zhenyuensis has previously only been tested in the phylogenetic analysis of Ezcurra \& Butler (2018). Like Fuyuansaurus acutirostris, it was recovered in a large polytomy within Tanystropheidae.

\section{Mesosuchus browni Watson 1912}

Age. Early Anisian, early Middle Triassic.

Occurrence. Burgersdorp Formation of the Beaufort Group near Aliwal North, Subzone B of the Cynognathus AZ, Eastern Cape Province, South Africa (Dilkes 1998; Hancox 2000).

Holotype. SAM-PK-5882, a partial skull consisting of the rostrum, braincase, and palatal regions, mandible, a partial vertebral column, an incomplete scapula and pelvic girdle, and partial fore and hindlimbs.

Referred specimens. The hypodigm of Mesosuchus browni was provided by Dilkes (1998) and was also listed by Ezcurra (2016).

Diagnosis. The most recent diagnosis was provided by Dilkes (1998).

Remarks. Mesosuchus browni is considered the best-known non-rhynchosaurid rhynchosaur (Butler et al. 2015; Hone \& Benton 2008). Like the other early rhynchosaurs Howesia browni and Eohyosaurus 
1410 wolvaardti, as well as the archosauriforms Euparkeria capensis and Erythrosuchus africanus, it is known

1411 from the Burgersdorp Formation near Aliwal North in the Eastern Cape, South Africa (Butler et al. 2015;

1412 Ezcurra et al. 2016; Rubidge 2005). The morphology of Mesosuchus browni has been studied several times 1413 (Broom 1913a; Broom 1913b; Broom 1925; Haughton 1922; Haughton 1924a; Watson 1912) and has been 1414 most comprehensively described by Dilkes (1998). In many ways Mesosuchus browni shows a morphology 1415 that is intermediate between that of rhynchosaurids and other early archosauromorphs. SAM-PK-6536 1416 represents a particularly informative specimen, as it includes a complete, virtually undistorted skull. The 1417 braincase of this specimen was recently described in detail by Sobral \& Müller (2019), which revealed the 1418 presence of a pneumatic sinus between the basal tubera. Pneumatization of the braincase was previously 1419 considered a derived archosaur trait, but its presence in Mesosuchus browni indicates it had evolved much 1420 earlier in the archosauromorph lineage.

Howesia browni Broom 1905

Age. Early Anisian, early Middle Triassic.

1424 Occurrence. Burgersdorp Formation of the Beaufort Group near Aliwal North, Subzone B of the Cynognathus AZ, Eastern Cape Province, South Africa (Dilkes 1995; Hancox 2000).

Holotype. SAM-PK-5884, a flattened partial skull missing the rostrum and occipital regions, and mandible.

Referred specimens. SAM-PK-5885, a flattened partial skull, mandible, and atlas-axis complex; SAM-PK5886, a postcranial skeleton consisting of a partial vertebral column, an incomplete pelvic girdle and left hindlimb, and a complete right tarsus.

Diagnosis. The most recent diagnosis was provided by Dilkes (1995).

Remarks. Howesia browni is a non-rhynchosaurid rhynchosaur closely related to Mesosuchus browni that was first described by Broom (1905a). Following additional preparation of the three known specimens, Howesia browni was extensively described by Dilkes (1995). 
1437 Occurrence. Burgersdorp Formation of the Beaufort Group near Aliwal North, Cynognathus AZ, Subzone 1438 B (Butler et al. 2015).

1439 Holotype. SAM-PK-K10159, skull and mandible missing the anterior part of the rostrum.

1440 Diagnosis. The diagnosis was provided by Butler et al. (2015).

1441 Remarks. Butler et al. (2015) described Eohyosaurus wolvaardti and included it in a phylogenetic analysis.

1442 It was recovered as the sister taxon to rhynchosaurids and was thus found to be more closely related to 1443 this clade than Mesosuchus browni and Howesia browni. In another phylogenetic analysis focusing on 1444 rhynchosaurs, it was found in a polytomy with rhynchosaurids, Mesosuchus browni, Howesia browni, and 1445 Noteosuchus colletti, the last being a poorly known early rhynchosaur from the Induan of South Africa 1446 (Ezcurra et al. 2016). However, more recent iterations of this data matrix corroborated the phylogenetic 1447 placement of Eohyosaurus wolvaardti by Butler et al. (2015) as the sister taxon to rhynchosaurids (e.g., 1448 Ezcurra 2016).

Pamelaria dolichotrachela Sen 2003

Age. Anisian, Middle Triassic (Lucas 2010).

Occurrence. Yerrapalli Formation, Gondwana Supergroup, Pranhita-Godavari Basin, southern India.

1453

1454

1455

1456

1457

1458

1459

1460

1461

1462
Holotype. ISIR 316, a partial skeleton including a largely complete skull.

Referred specimens. The referred specimens are listed in Sen (2003) and Ezcurra (2016).

Remarks. Pamelaria dolichotrachela is known from three specimens originating from the Yerrapalli Formation (Middle Triassic) of India and was originally identified as a "protorosaur" (Sen 2003). However, recent phylogenetic analyses have revealed that Pamelaria dolichotrachela is an allokotosaur that is closely related to Azendohsaurus spp. and Shringasaurus indicus (Ezcurra 2016; Nesbitt et al. 2015; Sengupta et al. 2017; and subsequent modifications of these matrices).

Azendohsaurus madagaskarensis Flynn, Nesbitt, Parrish, Ranivoharimanana \& Wyss 2010)

Age. Late Ladinian to early Carnian, late Middle Triassic to early Late Triassic.

Peer) reviewing PDF | (2020:08:51657:1:1:NEW 28 Feb 2021) 
1463 Occurrence. Locality M-28 close to the eastern bank of the Malio River, west of Isalo National Park, 1464 southern Madagascar, Isalo II or the Makay Formation (Nesbitt et al. 2015).

1465 Holotype. UA 7-20-99-653, partial skull and five anterior cervical vertebrae.

1466 Referred specimens. A list of referred specimens can be found in appendix 1 of Nesbitt et al. (2015).

1467 Diagnosis. The most recent diagnosis was provided in Nesbitt et al. (2015).

1468 Remarks. Azendohsaurus laaroussii is the type species of the genus, but Azendohsaurus madagaskarensis 1469 represents the best-known member of the genus. The former species was originally described from a few 1470 teeth and dental fragments and interpreted as an ornithischian dinosaur (Dutuit 1972) and later as a 1471 sauropodomorph dinosaur (e.g., Gauffre 1993). Postcranial remains from the type locality of 1472 Azendohsaurus laaroussii can likely also be referred to this species, and indicate that the taxon did not 1473 belong to Dinosauria (Cubo \& Jalil 2019; Jalil \& Knoll 2002). Comprehensive three-dimensionally preserved 1474 remains of various individuals from the late Middle Triassic to early Late Triassic of southern Madagascar, 1475 which closely resembled the known material of Azendohsaurus laaroussii, were assigned to 1476 Azendohsaurus madagaskarensis. The skull and mandible were initially described by Flynn et al. (2010). A 1477 description of the postcranium and a phylogenetic hypothesis for Azendohsaurus madagaskarensis was 1478 provided by Nesbitt et al. (2015). This revealed a new clade of non-archosauriform archosauromorphs, 1479 Allokotosauria, that includes Azendohsaurus spp., Trilophosaurus spp., Pamelaria dolichotrachela,

1480 Spinosuchus caseanus, and Teraterpeton hrynewichorum. Shringasaurus indicus was later also referred to 1481 this clade (e.g., Sengupta et al. 2017), which has also been recovered in subsequent phylogenetic analyses 1482 (e.g., Ezcurra 2016; Pritchard \& Sues 2019), confirming it as one of the three major lineages of non1483 archosauriform archosauromorphs previously recognized, together with Rhynchosauria and 1484 Tanystropheidae. Azendohsaurus madagaskarensis represents one of the best-known non1485 archosauriform archosauromorphs. It was herbivorous and has a relatively large body size among early 1486 archosauromorphs, being approximately 2 to 3 metres in length.

\section{Trilophosaurus buettneri Case 1928}

1489 Age. Early Norian, Late Triassic (Kligman et al. 2020).

1490 Occurrence. Trilophosaurus site 1 and Trilophosaurus quarries 1-3, Colorado City Formation; Walker's 1491 Tank and lower Kalgary site, Tecovas Formation, western Texas, USA (Spielmann et al. 2008). 
1492 Holotype. UMMP 2338, an incomplete right dentary bearing teeth.

1493 Referred specimens. A list of referred specimens can be found in appendix 1 of Spielmann et al. (2008).

1494 Diagnosis. The most recent diagnosis is provided by Spielmann et al. (2008, p. 11)

1495 Remarks. Trilophosaurus buettneri was first described based on a dentary fragment bearing teeth (Case 1496 1928) and interpreted to be closely related to procolophonids. Additional specimens that gave a much 1497 more complete account of the taxon were described by Gregory (1945). Gregory (1945) referred 1498 Trilophosaurus buettneri to "Protorosauria". The skull of Trilophosaurus buettneri was later redescribed 1499 by Parks (1969). Another species referred to the genus, "Trilophosaurus jacobsi", was proposed to 1500 represent a junior synonym of Spinosuchus caseanus by Nesbitt et al. (2015). Two additional species of 1501 the genus, Trilophosaurus phasmalophus and Trilophosaurus dornorum, are exclusively known from 1502 limited and isolated cranial or dental remains (Kligman et al. 2020; Mueller \& Parker 2006). Trilophosaurus 1503 buettneri was redescribed and reinterpreted as a non-archosauriform archosauromorph outside 1504 "Protorosauria" by Spielmann et al. (2008). Trilophosaurus buettneri was later found within the newly 1505 erected clade Allokotosauria (e.g., Ezcurra 2016; Nesbitt et al. 2015). The manus of Trilophosaurus 1506 buettneri was redescribed by Nesbitt et al. (2015). Trilophosaurus buettneri was herbivorous and has a 1507 remarkable dentition characterized by an edentulous, beak-like rostrum and labiolingually very wide 1508 tricuspid teeth further posterior in the jaws. An arboreal lifestyle has been suggested for Trilophosaurus 1509 buettneri by Spielmann et al. (2005), based on the relative proportions of the appendicular skeleton and 1510 the presence of large curved claws. Although similar claws occur in other allokotosaurs (e.g., 1511 Azendohsaurus madagaskarensis), a similar interpretation has not been made for these taxa (Nesbitt et 1512 al. 2015).

Teyujagua paradoxa Pinheiro, França, Lacerda, Butler \& Schultz 2016

Age. Induan-Olenekian, Early Triassic (Dias-da-Silva et al. 2017; Pinheiro et al. 2019).

1516 Occurrence. Bica São Tomé, Sanga do Cabral Formation, São Francisco de Assis, Rio Grande do Sul, 1517 southern Brazil.

1518 Holotype. UNIPAMPA 653, a nearly complete skull and mandible, as well as parts of the first five cervical 1519 vertebrae. 
1520 Diagnosis. The most recent diagnosis was provided by Pinheiro et al. (2019).

1521 Remarks. Teyujagua paradoxa was first described by Pinheiro et al. (2016) and subsequently described in 1522 further detail with the aid of $\mu C T$ data by Pinheiro et al. (2019). The skull and mandible of Teyujagua 1523 paradoxa exhibit a remarkable combination of plesiomorphic features, typical of non-archosauriform 1524 archosauromorphs (e.g., absence of the antorbital fenestra), and derived features that had previously 1525 been considered to represent synapomorphies for Archosauriformes (e.g., the presence of an external 1526 mandibular fenestra). This is also reflected in the position of Teyujagua paradoxa in previous phylogenetic 1527 analyses including this species, in which it was recovered as being very closely related to Archosauriformes 1528 (Pinheiro et al. 2016; Pinheiro et al. 2019).

Proterosuchus fergusi Broom 1903

Age. Induan, Early Triassic (Lucas 2010).

1532 1533

1534

1535

1536

1537 1538

1539

1540 1541

1542

1543

1544

1545

1546

1547

Occurrence. Several localities of the upper Balfour Formation and/or lower Katberg Formation, Lystrosaurus AZ, Karoo Supergroup in the Eastern Cape and Free State provinces of South Africa (a list of the exact localities is provided in table 3 of Ezcurra \& Butler, 2015b).

Neotype. RC 846 was assigned as the neotype of Proterosuchus fergusi by Ezcurra \& Butler (2015b), since the holotype SAM-PK-591, a partially preserved skull, is undiagnostic.

Hypodigm. A list of the referred specimens is presented in Ezcurra \& Butler (2015b, p. 164-165) and Ezcurra (2016, p. 47).

Diagnosis. The most recent emended diagnosis was provided by Ezcurra \& Butler (2015b).

Remarks. Proterosuchus fergusi is a medium-sized, predatory archosauriform characterized by a distinctly downturned premaxilla. The genus Proterosuchus, of which Proterosuchus fergusi is the type species, has a complicated taxonomic history and specimens have been referred to various species within the genus and the now synonymous genera "Chasmatosaurus" and "Elaphrosuchus". An extensive revision of the genus was provided by Ezcurra \& Butler (2015b), in which the species were distinguished based on a combination of cranial proportions and discrete characters. Quantitative investigation of cranial proportions was also used to investigate the ontogeny of Proterosuchus fergusi (Ezcurra \& Butler 2015a). A detailed morphological description of Proterosuchus fergusi has been provided by Cruickshank (1972; 
1548 Proterosuchus vanhoepeni therein). Recently, the endocast of Proterosuchus fergusi was described by 1549 Brown et al. (2019), which supported a semi-aquatic lifestyle for the taxon, although a histological and 1550 sedimentological study suggested a more terrestrial habit (Botha-Brink \& Smith 2011). Although the skull 1551 of Proterosuchus fergusi is represented by several, well-preserved specimens, the postcranium is 1552 comparatively much less well-known.

1553

1554

Proterosuchus alexanderi Hoffman 1965

1555

Age. Induan, Early Triassic (Lucas 2010).

1556

1557

1558

1559

1560

1561

1562

1563

1564

1565

1566

1567

1568

1569

1570

1571

1572

1573

1574 1575

Occurrence. Farm Zeekoegat close to Venterstad, upper Balfour Formation and/or lower Katberg Formation, Lystrosaurus AZ, Karoo Supergroup, Eastern Cape Province, South Africa (Ezcurra \& Butler 2015b; Hoffman 1965).

Holotype. NMQR 1484, a well-preserved specimen comprising a largely complete skull missing most of the premaxillae, and a postcranial skeleton missing part of the tail and the appendicular skeleton.

Diagnosis. The most recent diagnosis was provided by Ezcurra \& Butler (2015b).

Remarks. NMQR 1484 includes the best-preserved postcranial skeleton of the genus Proterosuchus. This specimen was first described by Hoffman (1965) and assigned to "Chasmatosaurus" alexanderi. This taxon was later synonymized with "Chasmatosaurus vanhoepeni" (e.g., Cruickshank 1972), which in turn was later synonymized with Proterosuchus fergusi (Welman 1998). However, the most recent revision of proterosuchid taxonomy (Ezcurra \& Butler 2015b) found NMQR 1484 to be taxonomically distinct from Proterosuchus fergusi, yet referrable to the same genus, and it was therefore reassigned as the only known specimen of the new combination Proterosuchus alexanderi. Proterosuchus alexanderi is included here as a separate OTU because its postcranial morphology is better known to that of the type species Proterosuchus fergusi.

\section{Euparkeria capensis Broom 1913}

Age. Early Anisian, early Middle Triassic.

Occurrence. Burgersdorp Formation of the Beaufort Group near Aliwal North, Subzone B of the Cynognathus AZ, Eastern Cape Province, South Africa (Ewer 1965; Hancox 2000; Sookias 2016). 
1576 Holotype. SAM-PK-5867, a complete skull and mandible and a largely complete and articulated postcranial 1577 skeleton, only missing most of the hands and feet and the majority of the tail.

1578 Referred specimens. A complete hypodigm is listed in Sookias (2016) and an updated specimen list of 1579 specimens preserving cranial elements is provided in Sookias et al. (2020).

Diagnosis. An emended diagnosis was recently provided in Sookias et al. (2020).

1581

1582

1583

1584

1585

1586

1587

1588

1589

1590

1591

1592

1593

1594

1595

1596

1597

1598

1599

1600

1601

1602

Erythrosuchus africanus Broom 1905

Age. Early Anisian, early Middle Triassic (Abdala et al. 2005).

Occurrence. Various localities in South Africa, most notably near Aliwal North and Burgersdorp, Eastern Cape Province, and Rouxville, Free State Province, Burgersdorp Formation of the Beaufort Group, Cynognathus AZ subzone B, Karoo Supergroup (Abdala et al. 2005; Gower 2003).

Holotype. SAM-PK-905, an incomplete postcranial skeleton, mainly consisting of the pectoral and pelvic girdles, partial forelimb, and vertebrae.

Referred specimens. A list of the referred specimens is provided in Appendix 1 of Gower (2003).

Diagnosis. The most recent diagnosis for Erythrosuchus africanus is provided by Ezcurra (2016).

Remarks. Erythrosuchus africanus is a large-sized carnivorous archosauriform with a skull that is particularly large compared to the postcranium. It was first described based on a partial postcranial skeleton by Broom (1905b). A new and more completely preserved specimen, including a partial skull, was described by Huene (1911). Erythrosuchus africanus was more recently described extensively by 
1603 Gower (2003), and separate studies addressed the morphology of the pes (Gower 1996), and braincase 1604 (Gower 1997).

1605

\section{Material and Methods}

1607

To resolve the phylogenetic relationships of tanystropheids and other "protorosaurs", a new comprehensive character matrix was constructed consisting of 307 characters. These include 40 new characters, with the remaining characters having been compiled and modified from the literature (mainly from Ezcurra 2016 and Pritchard et al. 2015, but also sourced from Benton \& Allen 1997; Dilkes 1998; Jalil 1997; Pritchard et al. 2018; Senter 2004; Simões et al. 2018; and Sookias 2016). The matrix contains 25 ratio characters, and 55 multistate characters are ordered as they are considered to form a transformational series in which at least one state represents a clear intermediate between two other states. Currently there is ongoing debate whether discrete characters should be ordered and whether to discretize continuous ratio data (e.g., Grand et al. 2013; Simões et al. 2016), but their application has been considered phylogenetically informative in previous studies of archosauromorph relationships (Ezcurra 2016; Nesbitt 2011). To test for the influence of ordered and ratio characters for our dataset, one round of analyses was performed including the ratio characters and with characters indicated as ordered treated as such, and another round without ratio characters and with all remaining characters treated as unordered. A total of 42 OTUs are included, the large majority of which were scored based on personal observations of relevant specimens. Petrolacosaurus kansensis was assigned as the outgroup. The continuous values calculated for the ratio characters are listed in the Supplementary Information for the sampled specimens of each OTU. These values were quantitatively partitioned into discrete character states using a classical cluster analysis in PAST 2.17c (Hammer et al. 2001). Following the methodology proposed by Ezcurra (2016), in the resultant tree a set of values were considered as a separate character state when the internal distance of that set was higher than the distance that separated it from (an)other value(s). In order to account for taphonomic deformation and other measurement uncertainties, character states that were separated by less than $5 \%$ of the total range present in the raw ratio values were merged. A full list of all character scorings and the specimens and literature that have been employed are provided in the Supplementary Information. The specimens that were scored are specified for each character individually for future assessment and comparison. 
Of the 42 included OTUs, Czatkowiella harae, Tanystropheus "conspicuus", and "Tanystropheus

1633

1634

1635

1636

1637

1638

1639

1640

1641

1642

1643

1644

1645

1646

1647

1648

1649

1650

1651

1652

1653

1654

1655

1656

1657

1658

1659

1660

1661

antiquus" can be considered problematic and their inclusion could therefore result in more ambiguous tree topologies. Czatkowiella harae is exclusively known from fragmented and isolated remains from the fissure deposits of Czatkowice, Poland (Borsuk-Białynicka \& Evans 2009b) and it is possible that multiple taxa are represented in the material (Ezcurra et al. 2014). The material currently known for Tanystropheus "conspicuus" is undiagnostic at the species level and this taxon is therefore currently considered a nomen dubium (Spiekman \& Scheyer 2019). "Tanystropheus antiquus" is currently insufficiently defined, since much of the type material that was considered to have been lost was recently rediscovered and is in need of revision (Skawiński et al. 2017; Spiekman \& Scheyer 2019).

Therefore, both analyses outlined above (one round excluding ratio characters and treating all characters as unordered, and one round including ratio and ordered characters) were performed once including all 42 OTUs, and once excluding Czatkowiella harae, Tanystropheus "conspicuus", and "Tanystropheus antiquus" a priori. Thus, in total four different analyses were performed. For the fourth analysis, which is the analysis including both ratio and ordered characters and which excluded the three problematic OTUs, we performed several heuristic searches in which specific constraints were defined (e.g., "Protorosauria" was enforced as a monophyletic group) to explore how many additional steps were required to obtain these alternative topologies.

The analyses were performed in TNT 1.5 (maximum parsimony criterion; Goloboff \& Catalano 2016), using several rounds of equally weighted 'New Technology Search' algorithms to adequately explore tree space and maximize the likelihood of finding the global optimum. Initial trees were calculated with 'Sectorial Search', 'Ratchet', 'Drift' and 'Tree Fusing' algorithms using 100 iterations each and 1000 random addition sequences (RAS). Relative fit difference was set at 0.1 and up to 10 suboptimal trees were retained. The saved trees were subsequently put through two separate analyses of three rounds each. One analysis applying 'Sectorial search', 'Ratchet', and 'Ratchet', in that order; and the other 'Ratchet', 'Sectorial search', and 'Ratchet'. All rounds ran 1000 iterations and additionally included 1000 iterations of 'Tree fusing'. At this stage suboptimal trees were discarded, and the strict consensus tree was calculated from the remaining trees. Branch support and stability were assessed with Bremer and Bootstrap support values, respectively. Bremer support values were calculated with the Bremer support function in TNT, using the absolute supports setting and TBR branch swapping on existing trees. Bootstrap support values were calculated using a 'Traditional search' at 1000 iterations. 
We employed the iter PCR function (Pol \& Escapa 2009) implemented in TNT to identify the

1663

1664

1665

1666

1667

1668

1669

1670

1671

1672

1673

1674

1675

1676

1677

1678

1679

1680

1681

1682

1683

1684

1685

1686

1687

1688

1689

1690

1691

presence of topologically unstable OTUs in the four different analyses we performed. The incorporation of these so called "wild card" OTUs results in polytomies in the strict consensus tree (SCT) derived from the most parsimonious trees (MPTs). The iter PCR function iteratively removes the unstable OTUs from the analyses a posteriori to calculate a number of reduced strict consensus trees (RSCTs) that are sequentially more resolved. This way we were able to assess the phylogenetic relationships between more stable OTUs that were obscured by the incorporation of the unstable OTUs.

\section{Character sampling and formulation}

Following detailed investigations of early archosaur phylogenetic relationships (e.g., Nesbitt 2011), the phylogeny of non-archosaurian archosauromorphs has received much attention in recent years and several detailed character lists for this group exist, with one analysis focusing on tanystropheids (Pritchard et al. 2015) and another on allokotosaurs (Nesbitt et al. 2015). However, the most comprehensive analysis, consisting of 600 characters, was provided by Ezcurra (2016), in which characters of these previous analyses were included, as well as those of many other studies. Furthermore, for many of the characters included at least one of the character states was figured, limiting subjective interpretation of the characters by the reader. Several subsequent studies have used and slightly modified the matrix provided by Ezcurra (2016) depending on the clade that was focused on in each respective study (e.g., Butler et al. 2019; Ezcurra et al. 2019; Maidment et al. 2020; Sengupta et al. 2017; Stocker et al. 2017).

Due to its comprehensiveness and well-explained characters, the character list of Ezcurra (2016) was used as the main source for our characters. However, only those characters that were relevant to the sampled taxa were included, as many characters in the original list were used to differentiate between taxa not included herein, such as proterochampsids, archosaurs, and choristoderans. Additional characters were incorporated mainly from the character list of Pritchard et al. (2015) and supplemented by characters from Pritchard et al. (2018), Nesbitt et al. (2015), Nesbitt (2011), Sookias (2016), Simões et al. (2018), Dilkes (1998), Jalil (1997), Benton \& Allen (1997), and Senter (2004). Certain characters taken from the literature were modified to fit more precisely with the specific morphologies observed in the sampled taxa. Finally, new characters were constructed based on detailed morphological comparisons of the included taxa. Certain autapomorphies for individual species were also incorporated into characters as they represent important morphological information and since these characters might prove 
1692 phylogenetically relevant in future studies. New characters and characters that have been distinctly 1693 modified from previous analyses are discussed and figured.

1694

1695

1696

1697

1698

1699

1700

1701

1702

1703

1704

1705

1706

1707

1708

1709

1710

1711

1712

1713

1714

1715

1716

1717

1718

1719

1720

1721

All characters were critically assessed on their logical construction and whether character states represent valid tests of similarity or primary homology. Issues regarding character construction, specifically how to optimize the construction of characters as to represent similarity tests, are a continuing source of debate (e.g., Brazeau 2011; Kearney \& Rieppel 2006; Rieppel \& Kearney 2007). Criteria for character construction to minimalize "non-meaningful" character scorings have been suggested by Simões et al. (2016) and examples of problematic characters have also been pointed out by Nesbitt (2011). We assessed all our characters in light of these suggestions, as these criteria provide important considerations for character construction. However, we find that the application of each criterion is dependent on various factors. The taxa included in our analysis all represent Permo-Triassic nonarchosaur members of the archosauromorph lineage, as well as early lepidosauromorphs and non-saurian diapsids. This phylogenetically relatively narrow sample is expected to exhibit less morphological variation than larger scale analyses (e.g., both extant and extinct Lepidosauromorpha and closely related taxa as in Simões et al. 2018). Therefore, in certain cases, characters that might otherwise not follow the criteria proposed in Simões et al. (2016) (e.g., the shape of the orbit, Type I A.7, or the use of continuous characters, Type II of Simões et al. 2016) are maintained when, based on detailed comparisons and careful consideration, the character states therein were deemed to likely represent valid similarity tests to the taxa involved. We pose that although these criteria as formulated represent useful tools, the complexity of morphological variation entails that careful observation and logical assessments of similarity by experts on the taxonomic sample at hand should be leading in character construction (Kearney \& Rieppel 2006;

Rieppel \& Kearney 2007). Therefore, a character that might be problematic as a test of homology when applied to one set of taxa, might still be valid when looking at a different taxonomic sample. Following Brazeau (2011), the presence or absence of a feature was formulated as a separate character from its morphology for unordered characters. In these cases, the character describing the morphology of this feature was scored as inapplicable in taxa in which the feature is absent.

Character list

1) Ezcurra (2016) ch. 20. Rostrum, antorbital length (anterior tip of the skull to anterior margin of the orbit) versus total length of the skull: 0.32-0.40 (0); 0.43-0.62 (1), RATIO (Ezcurra 2016: Figs. 17 and 18). 
1722 This character is considered to be interdependent with character 76 of Ezcurra (2016) for the taxonomic

1723 sample of our analysis. Since this character 20 could be applied to more taxa than character 76 , the

1724 former was preferred and the latter excluded.

1725 2) Ezcurra (2016) ch. $21 . \quad$ Rostrum, dorsoventral height at the level of the anterior tip of the

1726 maxilla versus dorsoventral height at the level of the anterior border of the orbit: 0.20-0.27 (0); 0.32-0.48

1727 (1); 0.56-0.78 (2), ORDERED RATIO (Ezcurra 2016: Figs. 17 and 19).3) Ezcurra (2016) ch. $22 . \quad$ Rostrum,

1728 proportions at the level of the anterior border of the orbit: transversely broader than dorsoventrally tall

1729 or subequal (0); dorsoventrally taller than transversely broad (1) (Ezcurra 2016: Fig. 16).

1730 4) Modified from Ezcurra (2016) ch. $27 . \quad$ Premaxilla, main body size: length of the tooth bearing

1731 margin in lateral view (in edentulous taxa the ventral margin of the premaxilla contributing to the

1732 ventral margin of the upper jaw; =main body) versus the length of the rostrum (anterior tip of the skull to

1733 the anterior border of the orbit): $0.09-0.10$ (0); 0.13-0.20 (1); 0.23-0.38 (2); 0.45-0.54 (3), ORDERED

1734 RATIO (Ezcurra 2016: Fig. 17).

1735 The original distinction between the character states was not considered to be phylogenetically relevant

1736 for the sampled taxa and therefore it was decided to distinguish states based on calculated ratios.

1737 5) Ezcurra (2016) ch. $29 . \quad$ Premaxilla, downturned main body: absent, alveolar margin sub-parallel

1738 to the main axis of the maxilla (0); slightly, in which the alveolar margin is angled at approximately 20

1739 degrees to the alveolar margin of the maxilla (1); strongly, prenarial process obscured by the postnarial

1740 process in lateral view (if the postnarial process is long enough) and postnarial process parallel or

1741 posteroventrally orientated with respect to the main axis of the premaxillary body (2), ORDERED (EzCUrra

1742 2016: Figs. 16-19).

1743 6) Ezcurra (2016) ch. $30 . \quad$ Premaxilla, angle formed between the alveolar margin and the anterior

1744 margin of the premaxillary body in lateral view: acute or right-angled (0); obtuse (1) (Ezcurra 2016: Figs.

174520 and 21). This character is inapplicable in taxa with a hooked premaxilla.

1746 7) Modified from Ezcurra (2016) ch. 34. Premaxilla, prenarial process: absent or incipient (0); present and

1747 less than the anteroposterior length of the main body of the premaxilla (1); present and longer than the

1748 anteroposterior length of the main body of the premaxilla (2), ORDERED (Ezcurra 2016: Figs. 17 and 21).

1749 This character is scored as inapplicable in taxa with confluent external nares. 
1750 An absent or incipient state was added and the character was ordered, since the character is considered

1751 a transformational series and state 1 represents a clear intermediate between states 0 and 2.

1752 Furthermore, the inapplicability criterion was included, since confluent external nares preclude the 1753 presence of a well-developed prenarial process.

1754 8) Modified from Ezcurra (2016) ch. $35 . \quad$ Premaxilla, base of the prenarial process:

1755 anteroposteriorly shallow, being not much wider at its base than further distally on the process (0);

1756 anteroposteriorly deep, being much wider at its base than further distally on the process (1) (Ezcurra

1757 2016: Figs. 12, 17, 20 and 21). This character is inapplicable in taxa that lack a prenarial process.

1758 This character was further clarified in its description and the inapplicability criterion has been modified.

1759 9) Modified from Ezcurra (2016) ch. 36 and 40. Premaxilla, postnarial process (=posterodorsal process, 1760 =maxillary process, =subnarial process): absent (0); short, ends well anterior to the posterior margin of 1761 the external naris (1); well-developed, forms most of the ventral border of the external naris or excludes 1762 the maxilla from participation in the external naris but process does not contact prefrontal (2); well1763 developed, forms most of the ventral border of the external naris and postnarial process of premaxilla 1764 contacts prefrontal (3), ORDERED (Ezcurra 2016: Figs. 17 and 19).

1765 Characters 36 and 40 of Ezcurra (2016) were combined here because a contact between the premaxilla 1766 and the prefrontal always requires the premaxilla to exclude the maxilla from the external nares.

1767 Therefore, these conditions can be considered as part of the same transformational series.

10) Ezcurra (2016) ch. 37. Premaxilla, postnarial process (=posterodorsal process, =maxillary process, =subnarial process): wide, plate-like (0); thin (1). This character is not applicable to taxa that lack a postnarial process (Ezcurra 2016: Fig. 20).

11) Modified from Ezcurra (2016) ch. 41, and Nesbitt et al. (2015): ch. $247 . \quad$ Premaxilla, plate-like palatal shelf or process on the medial surface (contribution to secondary palate by premaxillae): absent (0); present (1) (Ezcurra 2016: Figs. 12, 20 and 21)

1774 The character was redescribed to indicate that the "process" referred to represents a rather wide shelflike structure. For further explanation, see character 247 of Nesbitt et al. (2015). 12) Modified from Pritchard et al. (2015) ch. 1. Premaxilla, distinct posterodorsally to anteroventrally directed grooves terminating at the ventral margin of the bone: absent (0); present (1). 
1778 This character describes the presence of posterodorsally to anteroventrally directed grooves present in 1779 Langobardisaurus pandolfii MFSN 1921 (Saller et al. 2013) that were previously mistakenly identified as 1780 premaxillary teeth. This character was reformulated here to describe this feature more specifically.

1781 13) Modified from Ezcurra (2016) ch. 42 . Premaxilla, number of tooth positions: 8 or more (0); 5 or 1782 6 (1); 4 (2); 3 (3); 2 (4); 1 or edentulous (5) ORDERED (Ezcurra 2016: Figs. 16 and 17).

1783 The states of this character were modified since the original distinction did not cover all observed 1784 variation and because state 1 partially covered the same number of teeth as state 0 in the original 1785 description. Characters 69 and 278 of Ezcurra (2016) were omitted here, because we consider them to 1786 be strongly interdependent with this character for the current taxonomic sample.

1787 14) Ezcurra (2016) ch. $43 . \quad$ Premaxilla, orientation of the tooth series or the occlusal surface of 1788 premaxilla in ventral view: approximately parasagittal (0); strongly transverse and (in case of tooth1789 bearing premaxillae) anterior teeth covering each other in lateral view (1) (Ezcurra 2016: Fig. 21). This 1790 character is inapplicable in taxa with a hooked and beak-like premaxilla.

1791 The inapplicability criterion of this character was slightly modified. It was previously scored as 1792 inapplicable in taxa with an edentulous premaxilla. However, we consider that the differentiating 1793 morphology addressed by this character can also occur in taxa that lack premaxillary teeth. However, a 1794 hooked or beak-like shape morphology does not allow for a transverse occlusal surface and therefore 1795 taxa that exhibit this morphology should be scored as inapplicable for this character.

1796 15) Ezcurra (2016) ch. $24 . \quad$ Premaxilla-maxilla, suture: simple continuous contact (0); notched along 1797 the ventral margin (1) (Ezcurra 2016: Figs. 17 and 19).

1798 This character was also illustrated and discussed in Supplementary Figure 10 of Pritchard et al. (2018).

1799 However, we prefer the character formulation of character 24 of Ezcurra (2016) over that of Pritchard et 1800 al. (2018).

16) Modified from Ezcurra (2016) ch. 25.

Premaxilla-maxilla, subnarial foramen between the elements: absent (0); present (1) (Nesbitt 2011: Figs. 14, 17 and 19). This character is inapplicable in taxa 1803 that have a ventral notch on the suture of the premaxilla and maxilla.

1804 The inapplicability criterion was included because the presence of a ventral notch on the border 1805 between the premaxilla and maxilla precludes the possibility of a subnarial foramen. Character states 1 1806 and 2 of character 25 of Ezcurra (2016) were fused here because the distinction between these two 
1807 states is hard to establish confidently and because this distinction is most likely irrelevant to the taxon 1808 sample included in this analysis.

1809 17) New, similar to ch. 33 of Ezcurra (2016), ch. 6 of Pritchard et al. (2015), ch. 17 of Dilkes (1998), and 1810 ch. 6 of Pritchard et al. 2018. Premaxilla-maxilla, contact between the premaxilla and maxilla: simple 1811 abutting contact in which the premaxilla might overlap the maxilla slightly laterally (0); overlapping contact in which the maxilla considerably overlaps the premaxilla laterally (1); contact in which the premaxilla has a posteriorly directed peg on its posterolateral margin articulating with the maxilla, often accompanied by a groove (2); complex connection in which the premaxilla has posteriorly projected peg on its medial surface which locks the maxilla against the premaxilla medially (3) (Fig. 2).

The connection between the premaxilla and maxilla in early archosauromorphs has been discussed in depth and is considered phylogenetically informative. We have reformulated this character based on recent new findings with regards to the articulation between these elements in Macrocnemus bassanii (state 3; Miedema et al. 2020). Among the taxa sampled here, most have a simple abutting contact between premaxilla and maxilla, in which in many cases the premaxilla slightly overlaps the maxilla laterally (state 0; the sampled non-archosauromorph diapsids, Czatkowiella harae, Tanystropheus hydroides, Prolacerta broomi, Trilophosaurus buettneri, Teyujagua paradoxa, Euparkeria capensis, and Proterosuchus fergusi). In the sampled rhynchosaurs, the maxilla broadly overlaps the premaxilla laterally (state 1; Mesosuchus browni). Certain taxa bear a small peg, meaning a short pin or bolt, on the posterolateral end of their premaxilla, which connects to the lateral surface of the maxilla (state 2; Azendohsaurus madagaskarensis and Erythrosuchus africanus). The configuration of Macrocnemus bassanii, in which a peg is present on the medial side of the premaxilla that interlocks with an anteriorly facing peg on the medial side of the maxilla (Miedema et al. 2020), is considered to be morphologically distinct and almost certainly non-homologous to the pegs on the posterolateral end of the premaxilla described for state 2 and is therefore scored as a separate state here.

The presence of this small element in the anterior region of the rostrum is hard to establish, and therefore we were not able to confidently consider it as absent for any taxon. Therefore, this character is not phylogenetically informative for the present analysis, but it is nevertheless maintained as it presents an overview of the presence of the septomaxilla among early archosauromorphs. The elements 
1837 Mesosuchus browni by Dilkes (1998) can confidently be interpreted as the septomaxillae and are scored 1838 as such here.

1839 19) Ezcurra (2016) ch. 52, and Nesbitt et al. (2015): ch. $203 . \quad$ Maxilla, anterior maxillary foramen: 1840 absent (0); present (1) (Ezcurra 2016: Fig. 17).

1841

1842

20) Modified from Nesbitt et al. (2015): ch. 202.

Maxilla, dorsal portion, shape: gradual transition between the dorsal and posterior margin of the maxilla and no distinct process is formed (0); the dorsal apex of the maxilla ends abruptly and its posterior margin is concave (1) (Fig. 3).

The description of state 0 was modified to more precisely describe the condition observed in Youngina capensis and Protorosaurus speneri. The character as described by Nesbitt et al. (2015) was preferred over character 58 of Ezcurra (2016), since state 2 of the latter is likely strongly interdependent with the presence of an antorbital fenestra, which is scored here already in a separate character (22). In the description of character 202 of Nesbitt et al. (2015) it was pointed out that in Azendohsaurus madagaskarensis and Azendohsaurus laaroussii the posterodorsal margin of the maxilla is concave, which is similar to the condition in Archosauriformes in which this margin forms the anterior margin of the antorbital fenestra. A curved posterodorsal margin of the maxilla occurrs in many nonarchosauriform archosauromorphs (see also character 58 of Ezcurra 2016 and its scoring). The presence of a distinct ascending process of the maxilla, considered to be a saurian trait (sensu the scoring of character 57 in Ezcurra 2016), is considered to be too ambiguous as a phylogenetic character in the sampled taxa. For instance, the maxillae of Youngina capensis (AMNH FARB 5561), Protorosaurus speneri (NMK S 180), and Prolacerta broomi (BP/1/5880) do not bear a clearly defined process and the maxillae are approximately equally tall relative to their respective rostra in all three taxa. Nevertheless, the latter two taxa were previously considered to bear an ascending process, in contrast to the nonsaurian diapsid Youngina capensis. Furthermore, the presence of a process-like dorsal portion of the maxilla is strongly dependent on the relative height of the anterior portion of the rostrum at the level of the anterior margin of the orbit, and this morphology is already covered by character 10 here.

21) Modified from Ezurra (2016) ch. 59. Maxilla, anterior part of the dorsal margin: convex (0); straight (1); concave (2) (Ezcurra 2016: Fig. 22).

The anterior part of the dorsal margin is convex in Prolacerta broomi (BP/1/5880; Spiekman 2018), Trilophosaurus buettneri (TMM 31025-207), Protorosaurus speneri (NMK S 180), Mesosuchus browni (SAM-PK-6536), Youngina capensis (SAM-PK-K7578), Orovenator mayorum (OMNH 74606), 
1867 Petrolacosaurus kansensis (KUVP 9951), and Gephyrosaurus bridensis (Evans 1980). It is completely 1868 straight in Macrocnemus bassanii (PIMUZ T 4822) and Proterosuchus fergusi (RC 846; Ezcurra \& Butler 1869 2015b), which is here incorporated as a separate state, as it is considered homologous to neither the concave nor convex state, but instead represents an intermediate state.

22) Ezcurra (2016) ch. 13, and Pritchard et al. (2015): ch. 13. Antorbital fenestra: absent (0); present present (1) (Fig. 4). This character is inapplicable in taxa that have an antorbital fenestra.

A large circular concavity is present on the lateral margin of the maxilla of Dinocephalosaurus orientalis (IVPP V13767), which was referred to as an antorbital fossa by Rieppel et al. (2008). This character is clearly different from the antorbital fossa as described for Erythrosuchus (Gower 2003), which refers to a depression in the rostrum within which the antorbital fenestra is located. Because of the clear affinities with the antorbital fenestra, this antorbital fossa is considered to be non-homologous to the fossa described here, which we refer to as the maxillary fossa. It is currently only known to be present in Dinocephalosaurus orientalis. deflected from the main axis of the alveolar margin: absent (0); present (1) (Ezcurra 2016: Figs. 17 and 22). concave (1); convex (2); sigmoid, anteriorly concave and posteriorly convex (3); sigmoid, anteriorly convex, starting close to mid-length, and posteriorly concave (4) (Fig. 5; Ezcurra 2016: Figs. 16 and 19).

This character was modified to distinguish between a concave, straight, or convex margin, since it was considered that these distinctions might be phylogenetically informative for this sample of taxa.

This character is inapplicable in taxa with multiple tooth rows in the maxilla. 
1895

1896

1897

1898

1899

1900

1901

1902

1903

1904

1905

1906

1907

1908

1909

1910

1911

1912

1913

1914

1915

1916

1917

1918

1919

1920

1921

1922

1923

See the comments for character 28.

28) New, combination of ch. 47 of Ezcurra (2016) and part of ch. 8 of Pritchard et al. (2015). Maxillajugal, anguli oris crest: both the jugal and the maxilla are distinctly laterally offset (0); only the jugal is distinctly laterally offset (1) (Ezcurra 2016: Fig. 16). This character is scored as inapplicable in taxa that lack an anguli oris crest.

The term anguli oris crest is typically used to describe the very conspicuous lateral offset of the jugal seen in rhynchosaurid rhynchosaurs (e.g., Butler et al. 2015; Langer \& Schultz 2000; Montefeltro et al. 2010). This crest might have facilitated a muscular cheek (Benton 1983). A much less conspicuous anguli oris crest, which is partially formed by the posterolateral end of the maxilla, was recently described for the non-rhynchosaurid rhynchosaur Eohyosaurus wolvaardti (Butler et al. 2015). A similar lateral offset of the maxilla as seen in this taxon, which creates a substantial space between the lateral margin of the crest and the posterior portion of the maxillary tooth row, was considered for the trilophosaurid allokotosaurs Trilophosaurus buettneri and Teraterpeton hrynewichorum in character 8 of Pritchard et al. (2015). We consider the description of this character to address the same morphological structure as seen in Eohyosaurus wolvaardti and therefore fused the characters. A similar lateral offset might also be present in Langobardisaurus pandolfii. However, scoring this character for Langobardisaurus pandolfii is currently ambiguous since the preservation of this region is poor in the only specimen in which it is visible (MFSN 1921).

29) Ezcurra (2016) ch. 9. External nares, confluent: absent (0); present (1) (Ezcurra 2016: Fig. 16, 17 and 20).

30) Ezcurra (2016) ch. 12. External naris, shape: sub-circular (0); oval (1) (Ezcurra 2016: Fig. 19).

This character is inapplicable in taxa with confluent external nares.

An inapplicability criterion was added to this character.

31) New, similar to Ezcurra (2016) ch. 10. External naris: located close to the anterior end of the skull (0); a thick anterior margin of the premaxilla results in the external nares being posteriorly displaced (1) (Fig. 6).

In most taxa scored, the anterior margin of the external naris is closely positioned towards the anterior end of the rostrum. However, in the tanystropheids Tanystropheus hydroides (PIMUZ T 2790), Tanystropheus longobardicus (MSNM BES SC 1018), Macrocnemus bassanii (PIMUZ T 2477), and in 
1924 Pectodens zhenyuensis (IVPP V18578) and Dinocephalosaurus orientalis (IVPP V13767) the anterior 1925 margin of the external naris is separated considerably from the anterior end of the rostrum by the main 1926 body of the premaxilla.

1927 1928

32) Modified from Ezcurra (2016) ch. 78. Nasal, shape of anterior margin at midline: strongly convex with anterior process, and nasal forming a partial internarial bar (0); transverse with little convexity (1) (Ezcurra 2016: Fig. 16). This character is inapplicable in taxa in which the external nares are completely separated by an internarial bar.

This character is scored as inapplicable in taxa with a complete internarial bar, since this is always formed, at least in part, by an anterior process of the nasal. Thus, scoring taxa with a complete internarial bar for this character would result in overscoring this trait, as it is already addressed in character 5. Among the sampled taxa with confluent external nares, only the nasals of Azendohsaurus madagaskarensis bear clear anterior processes on the anteromedial margin of the nasal.

33) New. Nasal, antorbital recess: absent (0); or present (1) (Fig. 7).

The antorbital recess was described for Dinocephalosaurus orientalis by Rieppel et al. (2008). It is a large gully posterior to the external naris that is largely formed in the nasal bone, but the maxilla and possibly the prefrontal also contribute to it. This recess is non-homologous to the depression of the nasal described by character 80 of Ezcurra (2016). This recess has recently also been identified in Tanystropheus hydroides (Jiang et al. 2011; Spiekman et al. 2020a; Spiekman et al. 2020b).

34) Pritchard et al. (2018) ch. $311 . \quad$ Nasal, lateral surface: meets dorsoventrally short length of medial surface of dorsal process/portion of the maxilla (0); meets entire dorsoventral height of medial surface of supra-alveolar portion of maxilla (1). This character is inapplicable in taxa with an antorbital fenestra.

See character description of character 311 of Pritchard et al. (2018). The inapplicability criterion was added because it was considered that the presence of an antorbital fenestra implies that the nasal cannot have a wide articulation facet with the maxilla.

35) Dilkes (1998) ch. $15 . \quad$ Lacrimal, contacts nasal and reaches external naris (0); contacts nasal but does not reach naris (1); or does not contact nasal or reach naris (2), ORDERED. This character is inapplicable in taxa in which the premaxilla contacts the prefrontal. 
1952 This character was ordered because it is considered to represent a transformational series and state 1

1953 represents an intermediate between states 0 and 2. Furthermore, the inapplicability criterion was

1954 included, because a contact between the premaxilla and prefrontal precludes the possibility of a contact 1955 between the lacrimal and nasal.

1956 36) Ezcurra (2016) ch. $90 . \quad$ Lacrimal, naso-lacrimal duct position: opens on the posterolateral edge 1957 of the lacrimal (0); opens on the posterior surface of the lacrimal (1) (Ezcurra 2016: Fig. 19). This

1958

1959

1960

1961

1962

1963

1964

1965

1966

1967

1968

1969

1970

1971

1972

1973

1974

1975

1976

1977

1978

1979

1980

character is inapplicable if the prefrontal encloses part of the naso-lacrimal duct.

37) Ezcurra (2016) ch. 95. Jugal, anterior extension of the anterior process: anterior to the level of mid-length of the orbit (0); up to or posterior to the level of mid-length of the orbit (1).

38) Modified from Ezcurra (2016) ch. $92 . \quad J u g a l$, anterior process is dorsoventrally expanded anteriorly: absent, the anterior process tapers anteriorly and articulates with the dorsal surface of the posterior process of the maxilla (0); present, the anterior process of the jugal is expanded and partially covers the lateral surface of the posterior process of the maxilla (1) (Fig. 8).

In the majority of the taxa sampled here, the anterior process of the jugal fits into a groove or slot on the dorsal surface of the posterior process of the maxilla and is in some cases partially covered by the maxilla in lateral view. In the archosauriforms Euparkeria capensis (SAM-PK-5867) and Erythrosuchus africanus (BP///5207) the jugal is distinctly dorsoventrally taller and partially overlaps the maxilla in lateral view.

39) Modified from Sookias (2016) ch. 81. Jugal, bulges ventrolaterally at the point where its three processes meet: absent (0); present (1) (Fig. 8). This character is scored as inapplicable in taxa that lack a posterior process of the jugal.

This character describes the condition in the archosauriforms Proterosuchus fergusi (SAM-PK-11208), Erythrosuchus africanus (BP/I/5207), and Teyujagua paradoxa (Pinheiro et al. 2019). In these taxa the posterior process of the jugal is positioned further laterally than its anterior processes. This is caused by a lateral bulging of the jugal at the point where the anterior, posterior, and ascending/dorsal processes of the bone meet. This morphology is clearly distinct from the anteromedially to posterolaterally directed crest described as the anguli oris crest and therefore coded as a separate character.

40) Ezcurra (2016) ch. 98. Jugal, multiple pits on the lateral surface of the main body: absent (0); present (1) (Ezcurra 2016: Fig. 17).

Peer) reviewing PDF | (2020:08:51657:1:1:NEW 28 Feb 2021) 
1981 41) Ezcurra (2016) ch. $99 . \quad J u g a l$, ascending process forming the entire anterior border of the 1982 infratemporal fenestra: absent (0); present, postorbital excluded from the anterior border of the 1983 infratemporal fenestra (1) (Ezcurra 2016: Fig. 17). This character is inapplicable in taxa in which the 1984 anterior process of the squamosal possesses an extensive contact with the postorbital and contacts the 1985 jugal, and in taxa that lack an infratemporal fenestra or an ascending process on the jugal.

1986 42) New. Jugal, posterior process: present (0); absent (1) (Fig. 9).

1987 A posterior process, typically present in archosauromorphs, is completely absent in Claudiosaurus 1988 germaini (Carroll 1981), Pectodens zhenyuensis (IVPP V18578), Dinocephalosaurus orientalis (IVPP 1989 V13767), and Trilophosaurus buettneri (Spielmann et al. 2008).

43) Ezcurra (2016) ch. 100. Jugal, length of the posterior process versus the height of its base: $0.62-$ 2.28 (0); 2.64-3.64 (1); 4.48-4.74 (2); 5.29-5.84 (3), ORDERED RATIO (Fig. 9; Ezcurra 2016: Figs. 17 and 19). This character is inapplicable in taxa that lack a posterior process of the jugal. This character and character 42 (presence or absence of posterior process of the jugal) could also be treated as a single character. However, these characters were separated here because we omitted the ratio-based characters from some of our analyses. If characters 42 and 43 were combined the presence or absence of the posterior process of the jugal would be omitted from these analyses, despite representing a discrete rather than a continuous distinction.

44) Modified from Ezcurra (2016) ch. 5. Skull, dermal sculpturing on the dorsal surface of the frontals, parietals, and nasals: absent (0); shallow or deep pits scattered across surface and/or low ridges (1) (Ezcurra 2016: Fig. 16).

State 2 of character 5 in Ezcurra (2016) was not included here, because it was not applicable to the taxa 2003 sampled. transversely broad (0); present, nasal-frontal suture strongly transversely reduced (1) (Ezcurra 2016: Fig. 2006 7).

46) Ezcurra (2016) ch. 111 and Nesbitt et al. (2015) ch. 237. Prefrontal, lateral surface of the orbital 2008 margin: smooth or slight grooves present (0); rugose sculpturing present (1) (Ezcurra 2016: Fig. 17). 
2009 47) Modified from Ezcurra (2016) ch. 16. Orbit, shape: subcircular (0); distinctly dorsoventrally 2010 taller than long (1).

2011 Within the current taxonomic sample states 0 and 1 of character 16 in Ezcurra (2016) represent a 2012 relatively minor morphological difference of which the accurate observation is easily hampered by 2013 compression of specimens. Therefore, this character was modified to distinguish between the roughly 2014 subcircular orbits present in most of the sampled taxa, and the very dorsoventrally tall orbits of 2015 Proterosuchus fergusi (SAM-PK-11208), Proterosuchus alexanderi (NM QR 1484), and Erythrosuchus 2016 africanus (BP/1/5207).

2017 48) Modified from Ezcurra (2016) ch. $17 . \quad$ Orbit, elevated rim: absent or incipient (0); present, 2018 orbital margin of the jugal and/or postorbital slightly elevated to form a rim (1) (Ezcurra 2016: Figs. 16 2019 and 17).

2020 State 2 of character 17 in Ezcurra (2016) was excluded because it was not applicable to the sampled 2021 taxa.

2022 49) Modified from Ezcurra (2016) ch. 113. Frontal, suture with the nasal: transverse (0); oblique, 2023 forming an angle of at least 60 degrees with the long axis of the skull and frontals entering between both 2024 nasals (1); oblique and nasals entering considerably between frontals in a non-interdigitate suture (2); 2025 frontals enter nasals medially and nasals enter frontals laterally creating a $W$-shaped suture (3); frontals 2026 possess a three-pronged anteromedial process that articulates with the nasals (4) (Fig. 10; Ezcurra 2016 : 2027 Fig. 23 and Nesbitt 2011: Fig. 18). This character is inapplicable if the nasal is received by a slot in the 2028 frontal or the nasal does not contact the frontal.

2029 Character states 3 and 4 are new. State 3 is exhibited by Youngina capensis (BP/1/3859) and 2030 Tanystropheus hydroides (PIMUZ T 2787) and state 4 by Tanystropheus longobardicus (PIMUZ T 2484).

2031 50) Ezcurra (2016) ch. 114. (the character formulation has been slightly modified) Frontal, orbital 2032 border in skeletally mature individuals: absent or anteroposteriorly short and forms less than half of the 2033 dorsal edge of the orbit (0); anteroposteriorly long and forms at least more than half of the dorsal edge 2034 of the orbit (1) (Ezcurra 2016: Fig. 23).

2035 51) Ezcurra (2016) ch. 118. Frontal, dorsal surface adjacent to sutures with the postfrontal (if 2036 present) and parietal: flat to slightly concave (0); possesses a longitudinal and deep depression (1) 2037 (Ezcurra 2016: Fig. 16). 
2038 52) Ezcurra (2016) ch. 119. Frontal, longitudinal groove: longitudinally extended along most of the 2039 surface of the frontal (0); anterolaterally-to-posteromedially extended along the posterior half of the 2040 frontal (1) (Ezcurra 2016: Fig. 16). This character is inapplicable in taxa that lack a longitudinal 2041 depression on the frontal.

2042 The inapplicability criterion has been slightly modified.

2043 53) Ezcurra (2016) ch. 121 . Frontal, olfactory tract on the ventral surface of the frontal: maximum 2044 transverse constriction point well posterior to the moulds of the olfactory bulbs and posterolateral 2045 margin of the bulbs delimited by a low ridge (0); maximum transverse constriction of the olfactory tract 2046 immediately posterior to the moulds of the olfactory bulbs and posterolateral margin of the bulbs well2047 delimited by a thick, tall ridge (1) (Ezcurra 2016: Fig. 23). This character is inapplicable in taxa that lack 2048 olfactory bulb moulds and constriction of the olfactory tract canal.

2049 54) Ezcurra (2016) ch. 112 and Pritchard et al. (2015) ch. 14 . Frontal, frontals fused to one another: 2050 absent (0); present (1) (Ezcurra 2016: Fig. 23).

2051 This character should only be scored in skeletally adult specimens or in juvenile specimens in which the 2052 frontals have already fused.

2053 55) New. Frontal, width (or width of half of a fused frontal in taxa with fused frontals): narrow, 2054 frontal is considerably longer than wide (0); very wide and plate-like, frontal is almost as wide as long (1) 2055 (Fig. 11).

2056 This character describes the very wide frontals seen in Tanystropheus hydroides (PIMUZ T 2790) and 2057 Tanystropheus longobardicus (MSNM BES SC 1018).

2058 56) Pritchard et al. (2015) ch. 16 . Frontal, shape of contact with parietal in dorsal view: roughly 2059 transverse in orientation (0); frontals exhibit posterolateral processes, forming anteriorly curved U2060 shaped contact with parietals (1) (Ezcurra 2016: Figs. 8 and 23).

2061 This character is similar to character 116 in Ezcurra (2016). However, the version of this character 2062 formulated by Pritchard et al. (2015) is preferred because it is more specific to the taxon sample studied 2063 here.

2064 57) New, combining information from ch. 122 in Ezcurra (2016), ch. 15 in Pritchard et al. (2015), and ch. 2065313 in Pritchard et al. (2018). Postfrontal, suture with the frontal: anteroposteriorly or sagitally 
2066

2067

2068

2069

2070

2071

2072

2073

2074

2075

2076

2077

2078

2079

2080

2081

2082

2083

2084

2085

2086

2087

2088

2089

2090

2091

2092

2093

2094

2095

orientated (0); distinctly posteromedially inclined by a medial process of the postfrontal, resulting in posteriorly strongly narrowed frontal (1); distinctly posterolaterally inclined, resulting in a posteriorly expanded frontal and reduced postfrontal (2) (Fig. 12).

This character, describing the contact between the frontal and postfrontal, combines the description of several characters of previous analyses. In all non-archosauromorph diapsids included here, as well as in most non-archosauriform archosauromorphs (tanystropheids, Prolacerta broomi, Czatkowiella harae, and Protorosaurus speneri), the articulation between the postfrontal and frontal is sagitally orientated. However, in the rhynchosaurs Howesia browni and Mesosuchus browni, as well as the allokotosaurs Trilophosaurus buettneri and Azendohsaurus madagaskarensis, the postfrontal bears a distinct medial process, resulting in a posteriorly narrow frontal and a posteromedially orientated suture between the postfrontal and frontal. This morphology was described by character 313 of Pritchard et al. (2018). However, in the archosauriforms, a posteriorly wider frontal reduces the size of the postfrontal, as seen in Proterosuchus fergusi, Proterosuchus alexanderi, and Erythrosuchus africanus included here. This morphology was described by character 122 of Ezcurra (2016) as well as character 15 of Pritchard et al. (2015). In most archosaur groups, as well as Proterochampsia, the postfrontal has been lost completely (see character 44 of Nesbitt 2011). However, since no taxa belonging to these clades are included here, a separate character state referring to this condition has not been included.

58) New, combination of ch. 18 of Pritchard et al. (2015) and ch. 123 of Ezcurra (2016) (=ch. 27 of Pritchard et al. 2015). Postfrontal, lacks a posterior process and does not participate in the border of the supratemporal fenestra (0); has a posterior process and participates in the border of the supratemporal fenestra (1) (Ezcurra 2016: Fig. 16).

In all scored taxa, the presence of a posterior process of the postfrontal, or a roughly T-shaped postfrontal, implies that the postfrontal contributes to the margin of the supratemporal fenestra, and thereby prevents a contact between the postorbital and parietal (Youngina capensis, Gephyrosaurus bridensis, and Planocephalosaurus robinsonae). Therefore, we consider the contribution of the postfrontal to the supratemporal fenestra dependent on the presence of a T-shaped postfrontal, and we have combined the characters describing this morphology here.

59) Ezcurra (2016) ch. 124. Postfrontal, shape of dorsal surface: flat or slightly concave towards raised orbital rim (0); depression with deep pits (1) (Ezcurra 2016: Fig. 16). This character is inapplicable in taxa that lack a postfrontal. 
2096

2097

2098

2099

2100

2101

2102

2103

2104

2105

2106

2107

2108

2109

2110

2111

2112

2113

2114

2115

2116

2117

2118

2119

2120

2121

2122

2123

2124

60) Ezcurra (2016) ch. 130. Postorbital, posterior process extends close to or beyond the level of the posterior margin of the supratemporal fenestrae: absent (0); present (1) (Ezcurra 2016: Fig. 17).

61) Ezcurra (2016) ch. 131. Postorbital, extension of the ventral process: ends much higher than the ventral border of the orbit (0); ends close to or at the ventral border of the orbit (1) (Ezcurra 2016: Fig. 17).

62) Modified from Dilkes (1998) ch. 23. Postorbital, length of the ventral process versus the length of the posterior process of the postorbital: 0.47-0.59 (0); 0.76-0.88 (1); 0.99-1.17 (2); 1.33-1.62 (3); 1.78-1.95 (4); $2.08-2.20$ (5); 2.44-2.54 (6), ORDERED RATIO.

The identification of the processes was slightly modified for them to be congruent with other character descriptions listed here.

63) Ezcurra (2016) ch. 126. Postorbital-squamosal, upper temporal bar: located approximately at level of mid-height of the orbit (0); located approximately aligned to the dorsal border of the orbit (1) (Ezcurra 2016: Figs. 17 and 19). This character is inapplicable in taxa without an infratemporal fenestra and in taxa in which the upper temporal bar is very tall, reaching from the dorsal margin of the orbit to or beyond mid-height of the orbit.

An inapplicability criterion was added to this character, because in Trilophosaurus buettneri (Spielmann et al. 2008) the infratemporal fenestra is absent, and therefore an upper temporal bar is not present, and because in Tanystropheus hydroides (PIMUZ T 2790) the upper temporal bar is dorsoventrally tall and therefore covers the lateral side of the skull from the dorsal border of the orbit to about mid-height of the orbit, which covers both states of this character.

64) Modified from Ezcurra (2016) ch. 127. Postorbital-squamosal, contact: restricted to the dorsal margin of the elements (0); the anterior process of the squamosal continues along the posterior margin of the ventral process of the postorbital and contacts the jugal (1) (Nesbitt 2011: Figs. 17 and 19). This character is inapplicable in taxa that lack an infratemporal fenestra.

State 1 of character 127 in Ezcurra (2016) was not included here, because it is not applicable to any of the included taxa. An inapplicability criterion was included because it was considered that the morphology of Trilophosaurus buettneri (Spielmann et al. 2008), in which an infratemporal fenestra is absent, represents a distinctly separate morphology from state 0 , even though the squamosal and jugal likely did not meet in this taxon. 
2125 65) Ezcurra (2016) ch. $18 . \quad$ Infratemporal fenestra: present (0); absent (1).

2126 66) Ezcurra (2016) ch. $137 . \quad$ Squamosal, anterior process forms more than half of the lateral border

2127 of the supratemporal fenestra: absent (0); present (1) (Ezcurra 2016: Fig. 16). This character is

2128 inapplicable in taxa lacking a supratemporal fenestra.

2129 67) Modified from Ezcurra (2016) ch. 143 and Pritchard et al. (2015) ch. $33 . \quad$ Squamosal, ventral 2130 process: present (0); absent or completely confluent with anterior process (1) (Fig. 13).

2131 This character was modified based on the observed morphologies in the sampled taxa. In Tanystropheus

2132 hydroides (PIMUZ T 2790) no clear ventral process can be distinguished, but instead the anterior process 2133 of the squamosal is dorsoventrally tall and plate-like. This is possibly the result of a confluence of the 2134 anterior and ventral processes (Spiekman et al. 2020b). In Trilophosaurus buettneri (Spielmann et al. 2135 2008) the ventral process is also absent.

2136 68) Ezcurra (2016) ch. 139 (slightly reformulated). Squamosal, ventral process: angle between the 2137 ventral and anterior processes of the squamosal 90 degrees or less, forming a roughly square outline (0); 2138 angle between the ventral and anterior processes of the squamosal more than 90 degrees, forming a 2139 gentle, widely rounded posterodorsal border of the infratemporal fenestra (1) (Ezcurra 2016: Figs. 8, 17, 214018 and 24). This character is scored as inapplicable in taxa that lack a ventral process of the squamosal. 69) Pritchard et al. (2015) ch. 34 Squamosal, ventral process: forming a massive flange that 2142 covers the quadrate entirely in lateral view (0); anteroposteriorly slender (1). This character is scored as inapplicable in taxa that lack a ventral process on the squamosal.

2144 See character description of character 135 of Ezcurra (2016), which covers the same distinction. The 2145 character description of Pritchard et al. (2015) was preferred here, because it is considered to be more 2146 informative. The inapplicability criterion has been added.

2147 70) Ezcurra (2016) ch. 140. Squamosal medial process: short, forming up to half or less of the 2148 posterior border of the supratemporal fenestra (0); long, forming entirely or almost entirely the posterior 2149 border of the supratemporal fenestra (1) (Ezcurra 2016: Fig. 16). This character is scored as inapplicable 2150 in taxa that lack a medial process of the squamosal.

2151 The inapplicability criterion was added. 
2152 71) New. Squamosal medial process, dorsoventrally short (0); dorsoventrally tall and plate-like,

2153 forming a tall surface of the posterior margin of the supratemporal fenestra (1) (Fig. 13). This character

2154 is scored as inapplicable in taxa that lack a medial process of the squamosal.

2155 72) Modified from Ezcurra (2016) ch. 141. Squamosal, posterior process is distinct and extends 2156 posterior to the dorsal head of the quadrate: absent (0); present (1) (Ezcurra 2016: Fig. 18, 19, and 24).

2157 This character is inapplicable in taxa where the quadrate is completely covered by the squamosal in 2158 lateral view.

2159 The description of this character was modified to more clearly describe the morphology observed in the 2160 taxon sample studied here.

2161 73) Ezcurra (2016) ch. $157 . \quad$ Supratemporal: broad element (0); slender, in parietal and squamosal 2162 trough (1); absent (2) ORDERED (Ezcurra 2016: Fig. 17).

2163 The definitive absence of the supratemporal is hard to establish because it is often a small element that 2164 is easily obscured by specimen disarticulation or compression. Therefore, following Ezcurra (2016), this 2165 bone is only scored as absent when it can be confidently established as such from well-preserved 2166 specimens.

2167 74) Ezcurra (2016) ch. 159 and Pritchard et al. (2015) ch. $19 . \quad$ Parietal, median contact between both 2168 parietals: suture present (0); fused with loss of suture (1) (Ezcurra 2016: Fig. 16).

2169 State 0 can only be scored based on skeletally mature specimens.

2170 75) Ezcurra (2016) ch. 160. Parietal, extension over interorbital region: absent or slight (0); present 2171 (1) (Ezcurra 2016: Figs. 6 and 23).

2172 76) Ezcurra (2016) ch. 162. Parietal, pineal fossa on the median line of the dorsal surface: absent (0); 2173 present (1). This character should not be scored for early juveniles (Fig. 14; Ezcurra 2016: Fig. 8).

2174 This character was considered to be present in Kadimakara australiensis and several archosauriforms 2175 (see scorings of Ezcurra 2016, character 162). However, a similar fossa as present in these taxa can also 2176 be identified in Azendohsaurus madagaskarensis (Flynn et al. 2010), Trilophosaurus buettneri (Flynn et 2177 al. 2010), and Dinocephalosaurus orientalis (IVPP V13767).

2178 77) Modified from Ezcurra (2016) ch. $164 . \quad$ Parietal, pineal foramen in dorsal view: large (0); 2179 reduced to a small, circular pit or concavity (1); absent (2) (Ezcurra 2016: Figs. 6 and 8), ORDERED. 
2180 The character was ordered, since it is considered a transformational series in which state 1 represents a

2181 clear intermediate between states 0 and 2. Furthermore the concavity statement was added to state 1 , 2182 because in some taxa this depression is not pit-like.

2183 78) Modified from Ezcurra (2016) ch. 165 . Parietal, position of the pineal foramen in dorsal view:

2184 enclosed by parietals and clearly on the posterior part of the bones (0); enclosed by parietals at roughly

2185 mid-length of the bones (1); enclosed by parietals on the anterior part of the bones close to the frontals

2186 (2); enclosed by both frontals and parietals (3), ORDERED (Ezcurra 2016: Figs. 6 and 8). This character is

2187 scored as inapplicable in taxa that lack a pineal foramen.

2188 The pineal foramen is displaced distinctly posteriorly on the parietals in the non-archosauromorph 2189 diapsids Planocephalosaurus robinsonae (Fraser 1982), Orovenator mayorum (Ford \& Benson 2018), and 2190 Youngina capensis (AMNH FARB 5561), and this was therefore considered as a separate character state.

2191 This morphology was extensively discussed in Ford and Benson (2018, p. 208).

2192 79) Modified from Pritchard et al. (2015) ch. $21 . \quad$ Parietal, orientation of the posterolateral 2193 process: roughly transverse (0); strongly angled posterolaterally (1) (Fig. 14).

2194 In most of the sampled taxa, the posterolateral processes have a posterolateral orientation. However, in 2195 Tanystropheus hydroides (PIMUZ T 2819), Tanystropheus longobardicus (PIMUZ T 2484), Protorosaurus speneri (NMK S 180), and Azendohsaurus madagaskarensis (Flynn et al. 2011), the posterolateral process has a completely transverse or lateral orientation.

80) Ezcurra (2016) ch 168. Parietal, posterolateral process height: dorsoventrally low, usually considerably lower than the supraoccipital (0); dorsoventrally deep, being plate-like in occipital view and subequal to the height of the supraoccipital (1) (Ezcurra 2016: Fig. 27).

2201

81) Modified from Ezcurra (2016) ch. 8. Parietal, supratemporal fossa medial to the supratemporal fenestra: absent (0); present (1) (Fig. 14). fossa medial to the supratemporal fenestra: well-exposed in dorsal view and mainly dorsally or dorsolaterally facing (0); poorly exposed in dorsal view and mainly laterally facing (1) (Ezcurra 2016: Fig. 16). This character is scored as inapplicable in taxa that lack a supratemporal fossa on the parietal. 
2209 table (0); expanded distinctly medially, resulting in a mediolaterally narrow parietal table (1);

2210 supratemporal fossae strongly expanded medially and only separated by a ridge running along the

2211 midline of the parietal, the sagittal crest (2), ORDERED (Fig. 14). This character is scored as inapplicable

2212 in taxa that lack a supratemporal fossa on the parietal.

2213 This character was modified to clarify the distinction between the different states. The supratemporal

2214 fossa can either be restricted to the lateral portion of the parietal, expressed more widely on the

2215 parietal, or cover most of the dorsal surface of the parietal between the supratemporal fenestrae, only

2216 leaving a thin sagittal crest between the two fossae. This character is very variable in Prolacerta broomi

2217 with all three states observed in different specimens (state 0: BP/1/471, state 1: BP/1/5375 and UCMP

221837151 , state $2: B P / 1 / 5066$ and $B P / 1 / 5880)$.

84) Ezcurra (2016) ch. 171.

Postparietal, size (pair of postparietals if they are not fused to each other): sheet-like, not much narrower than the supraoccipital (0); small, splint-like (1); absent as a separate ossification (2) ORDERED (Ezcurra 2016: Fig. 23).

85) Ezcurra (2016) ch. 172 and Pritchard et al. (2015) ch. 25. Postparietal, fusion between counterparts: absent (0); present, forming an interparietal (1). This character is inapplicable in taxa that lack postparietals.

86) Ezcurra (2016) ch. 173 and Pritchard et al. (2015) ch. $37 . \quad$ Tabular: present (0); absent (1).

87) Ezcurra (2016) ch. 150 and Pritchard et al. (2015) ch. 38. Quadratojugal: absent or fused to the quadrate (0); present (1) (Ezcurra 2016: Fig. 24). margin of the bone rounded and the quadratojugal and jugal do not connect and therefore the lower temporal bar is incomplete (0); incipient, short anterior prong on the anteroventral margin of the bone and the quadratojugal and jugal connect and therefore the lower temporal bar is complete (1); distinctly present, in which the lower temporal bar is complete, but process terminates well posterior to the base of the posterior process of the jugal (2); distinctly present, in which the lower temporal bar is complete and participates in the posteroventral border of the infratemporal fenestra, and process terminates close to the base of the posterior process of the jugal (3), ORDERED (Ezcurra 2016: Figs. 17 and 19). This character is inapplicable in taxa that lack an infratemporal fenestra or quadratojugal. 
2237 The description of this character was modified to more clearly describe the morphology observed in the 2238 taxon sample studied here.

2239 89) Ezcurra (2016) ch. 156 . Quadratojugal, posterior extension of the ventral end: absent, without a 2240 posteriorly arched quadratojugal (0); limited, ventral condyles of the quadrate broadly visible in lateral 2241 view (1); strongly developed, overlapping completely or almost completely the ventral condyles of the 2242 quadrate in lateral view (2), ORDERED (Ezcurra 2016: Fig. 18). This character is inapplicable in taxa 2243 lacking a quadratojugal.

$224490)$ New, combination of ch. 176 and ch. 182 in Ezcurra (2016). Quadrate, posterior margin in lateral 2245 view: straight along entire shaft (0); continuously concave (1); sigmoidal, with a concave dorsal portion 2246 and convex ventral portion (2) (Ezcurra 2016: Fig. 24).

2247 Characters 176 and 182 of Ezcurra (2016) were combined because they both relate to the shape of the 2248 quadrate shaft. The presence of a quadrate conch is omitted because its presence is likely closely related 2249 to a fusion of the quadratojugal to the quadrate in lepidosauromorphs. This fusion is already coded for 2250 by character 87 and its inclusion here would result in the overscoring of this morphology.

2251 91) Ezcurra (2016) ch. 180 and Nesbitt et al. (2015) ch. 207. Quadrate, dorsal end hooked posteriorly 2252 in lateral view: absent (0); present (1) (Ezcurra 2016: Figs. 17 and 24).

2253 92) New. Quadrate, ventral condyles: lateral and medial condyles not distinctly separated and 2254 therefore the ventral surface of the quadrate is rounded, flat, or slightly concave (0); condyles separated 2255 by a deep concavity on the ventral surface of the quadrate (1) (Fig. 15).

2256 93) Ezcurra (2016) ch. 183. Quadrate, ventral condyles: subequally distally extended (0); medial 2257 condyle distinctly more distally projected than the lateral one (1) (Fig. 15).

2258 94) New. Quadrate, pterygoid flange: anteriormost extension at about mid-height of the quadrate 2259 shaft (0); dorsally located, the anteriormost extension of the flange is at close to the dorsoventral level of 2260 the dorsal head of the quadrate (1) (Fig. 15).

2261 This character describes the difference seen in the morphology of the pterygoid flange, as can be clearly 2262 observed between for instance Tanystropheus hydroides (PIMUZ T 2790) and Macrocnemus bassanii 2263 (PIMUZ T 2477).

2264 95) Pritchard et al. (2015) ch. $45 . \quad$ Vomer, teeth: absent (0); present (1). 
2265

2266

2267

2268

2269

2270

2271

2272

2273

2274

2275

2276

2277

2278

2279

2280

2281

2282

2283

2284

2285

2286

2287

2288

2289

2290

2291

2292

96) Modified from Ezcurra (2016) ch. 187.

Vomer, teeth distribution: shagreen tooth distribution

with no clear rows distinguishable (0); teeth distributed in multiple clearly defined rows (1); teeth distributed mainly in a single row, but multiple teeth present immediately anterior to the contact with the pterygoid (2); teeth distributed in a single row along entire extension (3). This character is inapplicable in taxa that lack vomerine teeth.

The presence of vomerine teeth and their distribution were considered in one ordered character in character 187 in Ezcurra (2016). However, we do not consider any of the various tooth distributions to represent an intermediate stage between any of the others. Therefore, we treat the presence of vomerine teeth as a separate character, and the distribution of these teeth, if they are present, as a separate, unordered character.

97) New, related to ch. 189 of Ezcurra (2016). Palatal dentition, size (height and diameter) of teeth on the vomer: small, considerably smaller than those of the marginal dentition (0); relatively large, similar to those of the marginal dentition (1) (Fig. 16). This character is inapplicable in taxa lacking vomerine teeth.

Character 189 in Ezcurra (2016) describes the relative size of the teeth on the palatine and pterygoid. However, in our sampled taxa, a distinct difference in the size of the dentition could also be observed in the vomer, and this was therefore formulated into a separate character, since the size of the vomerine teeth does not appear to be dependent on the size of the palatine or pterygoid teeth in the sampled taxa.

98) Ezcurra (2016) ch. 190 (description of state 1 slightly reformulated). Palatine, transverse extension: narrow, subequal contribution of the palatine and pterygoid to or pterygoid main component of the palate posterior to the choanae (0); broad, the palatine is the main component of the palate posterior to the choanae (1) (Ezcurra 2016: Fig. 26).

99) Modified from Ezcurra (2016) ch. 191 . Palatine, anterior processes forming the posterior border of the choana: subequal in anterior extension or anterolateral process longer (0); anteromedial process longer (1) (Ezcurra 2016: Fig. 26).

Character state 2 of character 191 in Ezcurra (2016) was not included here, because it is not applicable to any of the sampled taxa. 
2293 100) Ezcurra (2016) ch. 188.

Palatine-pterygoid, teeth on the palatine and ventral surface of

2294 the anterior ramus of the pterygoid: present (0); absent (1) (Ezcurra 2016: Figs. 13, 24 and 26).

2295

101) Part of Ezcurra (2016) ch. 189. Palatal dentition, size (height and diameter) of teeth on the

2296 palatine: small, considerably smaller than those of the marginal dentition (0); relatively large, similar to those of the marginal dentition (1) (Fig. 16; Ezcurra 2016: Figs. 25 and 26). This character is inapplicable in taxa lacking palatine teeth.

Character 189 in Ezcurra (2016) treats the size of the dentition on the palatine and pterygoid as a single character. Because the relative size of the teeth on the palatine and pterygoid differs in Tanystropheus longobardicus (PIMUZ T 2484) it was decided here to treat the size of the teeth on both elements as separate characters.

102) Ezcurra (2016) ch. 195. Pterygoid, teeth on the ventral surface of the anterior ramus (=palatal process), excluding tiny palatal teeth if present: present in two distinct fields (=T2 and T3 of Welman 1998) (0); present in three distinct fields ( $=T 2, T 3 a$ and T3b) (1); present in three distinct fields $(=T 2 a, T 2 b$ and T3) (2); present in one field that occupies most of the transverse width of the ramus (=T2 + T3) (3); present in only one posteromedially-to-anterolaterally orientated field (=T2) (4); present in only one field adjacent to the medial margin of the ramus (=T3) (5); present in no definable fields but the entire pterygoid is covered by a shagreen of teeth (6) (Ezcurra 2016: Figs. 25 and 26). This character is inapplicable in taxa that lack teeth in the palatine and the ventral surface of the anterior ramus of the pterygoid.

103) Ezcurra (2016) ch. 196. Pterygoid, number of rows on palatal tooth field T2: more than two or do not dispose on distinct rows (0); two rows parallel to each other (1); single row (2) (Ezcurra 2016: Figs. 25 and 26). This character is inapplicable if the tooth field $T 2$ is subdivided in $T 2 a$ and $T 2 b$ or is absent. tooth field T3: more than two or not placed in distinct rows (0); two parallel rows (1); single row (2)

2317 (Ezcurra 2016: Figs. 25 and 26). This character is inapplicable if the tooth field T3 is subdivided into T3a and $T 3 b$ or is absent.

2319 Character 199 in Ezcurra (2016) treats a row of teeth sticking out on the medial side of the anterior ramus of the pterygoid (=T4 of Welman 1998) as a separate character. It is found here, based on observations of Macrocnemus bassanii (PIMUZ T 1559) and Prolacerta broomi (unpublished CT-scan of $\mathrm{BP} / 1 / 5066$ ) that tooth field T3 in these taxa bears more than two distinct rows. Furthermore, the medial 
2323 margin of the anterior ramus of the pterygoid is curved, resulting in a number of these teeth facing

2324 lateroventrally, whilst others face mediolaterally. Therefore, we conclude that tooth field T4 actually

2325 represents the mediolaterally facing teeth of tooth field T3 and consequently character 199 in Ezcurra

2326 (2016) has not been included here.

2327 105) Part of Ezcurra (2016) ch. 189. Palatal dentition, size (height and diameter) of teeth on the 2328 ventral surface of the anterior ramus of the pterygoid: small, considerably smaller than those of the 2329 marginal dentition (0); relatively large, similar to those of the marginal dentition (1) (Fig. 16; Ezcurra 2330 2016: Figs. 25 and 26). This character is inapplicable in taxa lacking teeth on the anterior ramus of the 2331 pterygoid.

2332 See description of character 101.

106) Part of Ezcurra (2016) ch. 202. Pterygoid, teeth on the lateral ramus (=transverse flange): absent (0); present (1) (Ezcurra 2016: Figs. 13, 25 and 26). This character is inapplicable in taxa in which most of the pterygoid is covered by shagreen teeth.

The inapplicability criterion was added because this tooth row cannot be distinguished from other pterygoid teeth when the pterygoid is covered by shagreen teeth. We separated this character from character 107 because we consider the presence of teeth on the lateral ramus of the pterygoid to represent a separate criterion from the number of tooth rows if such teeth are present. Therefore, we do not consider the presence of a single row of teeth to represent an intermediate step in a transformational series between no teeth present and two rows present. flange): teeth distributed in a single row on the posterior edge (=T1 of Welman 1998) (0); teeth distributed in multiple rows (1) (Ezcurra 2016: Figs. 13, 25 and 26). This character is inapplicable in taxa that lack teeth on the lateral ramus of the pterygoid or in taxa in which shagreen teeth cover the pterygoid.

2347 See description of character 107.

2348 108) New. Pterygoid, anterior end of the anterior ramus: tapers to an end (0); rounded (1) (Fig. 16).

2349 In most of the sampled taxa, the anterior ramus of the pterygoid gradually tapers anteriorly and thus 2350 has an anteriorly pointed end. In contrast, in Tanystropheus hydroides (PIMUZ T 2787) and 
2351 Dincephalosaurus orientalis (Rieppel et al. 2008) the anterior ramus of the pterygoid is much wider 2352 anteriorly and has a rounded anterior margin.

2353 109) New. Pterygoid, lateral/distal end of the posterior margin of the lateral ramus

2354 (=transverse flange) curved posteriorly: absent (0); present (1) (Fig. 16). This character is scored as 2355 inapplicable in taxa with a strongly posterolaterally orientated lateral ramus of the pterygoid.

2356 This character is closely related to character 201 in Ezcurra (2016). However, because this new 2357 description distinguishes between morphologies seen in tanystropheids, it is considered to be more 2358 informative and therefore preferred. Character 201 of Ezcurra (2016) was not included in order to 2359 prevent overscoring of this morphology.

110) Modified from Ezcurra (2016) ch. 207. Ectopterygoid, lateral process is not curved posteriorly (0); lateral process is curved posteriorly but not expanded posteriorly (1); lateral process is both curved and expanded posteriorly, giving the ectopterygoid a hook-shape in dorsal or ventral view (2) (Fig. 16; Ezcurra 2016: Figs. 7 and 26), ORDERED.

The lateral portion of the ectopterygoid can be separated into three different morphologies. In some taxa, it is not curved, nor expanded (e.g., Azendohsaurus madagaskarensis, Flynn et al. 2010). In other taxa, the lateral end curves posteriorly but it is not expanded anteroposteriorly (e.g., Macrocnemus bassanii, Miedema et al. 2020). Finally, in certain taxa, the lateral portion of the ectopterygoid is curved posteriorly and is expanded anteroposteriorly (e.g., Orovenator mayorum, Ford \& Benson 2018). Since state 1 is considered to represent an intermediate state between 0 and 2 in a transformational series, this character was ordered. ectopterygoid overlaps the pterygoid ventrally (0); interlaced articulation, complex articulation between ectopterygoid and pterygoid (1) (Ezcurra 2016: Fig. 26).

112) Ezcurra (2016) ch. 205 (the formulation of this character has been modified slightly). Ectopterygoid, connection with pterygoid: does not reach the posterolateral corner of the lateral ramus (=transverse flange) (0); reaches the posterolateral corner of the lateral ramus (1) (Ezcurra 2016: Fig. 26). This character is scored as inapplicable in taxa in which the ectopterygoid simply overlaps the pterygoid. 
2379 An inapplicability criterion is added because the ectopterygoid only reaches the posterolateral corner of

2380 the lateral ramus of the pterygoid when the ectopterygoid forms an interlacing suture with the

2381 pterygoid. In taxa with this type of articulation, the ectopterygoid wraps around the posterolateral

2382 corner of the transverse flange in some cases.

2383 113) Ezcurra (2016) ch. 244 and Pritchard et al. (2015) ch. 65. Parasphenoid/parabasisphenoid,

2384 dentition on cultriform process: present (0); absent (1).

2385 114) New. Parasphenoid/parabasisphenoid, length of the cultriform process versus its height at its 2386 anteroposterior midpoint: 4.16-5.77 (0); 9.65-9.89 (1); 10.85-12.12 (2); 13.29-13.42 (3); 20.28-21.12 (4),

2387 ORDERED RATIO.This character covers the large discrepancy in the relative length of the cultriform

2388 process. In most taxa it is a thin elongate element, whereas in allokotosaurs and rhynchosaurs it is much 2389 shorter and dorsoventrally taller.

2390 115) New. Parasphenoid/parabasisphenoid, anterior projections of the cristae trabeculares, present 2391 (0); absent (1).

2392 The cristae trabeculares are small bony projections on the anterolateral surface of the cultriform 2393 process of the parabasisphenoid, which occur in certain non-saurian diapsids and lepidosaurs. These 2394 structures and their occurrence among diapsids were discussed in detail by Ford and Benson (2018, 2395 page 18).

2396 116) Ezcurra (2016) ch. 236. Parasphenoid/parabasisphenoid, posterodorsal portion: incompletely 2397 ossified (0); completely ossified (1).

2398 117) Modified from Ezcurra (2016) ch. 237. Parasphenoid/parabasisphenoid, intertuberal plate: 2399 present (0); absent (1) (Ezcurra 2016: Figs. 10 and 28).

2400 Character states 1 and 2 of character 237 in Ezcurra (2016) were fused here, because there was no clear 2401 distinction between a rounded and a straight posterior edge of the intertuberal plate in the sampled 2402 taxa, and this distinction is likely only relevant in more derived archosauriforms.

2403 118) Modified from Ezcurra (2016) ch. 239. Parasphenoid/parabasisphenoid, recess (=median 2404 pharyngeal recess, =hemispherical sulcus, =hemispherical fontanelle): absent, the ventral floor of the 2405 parabasisphenoid posterior to the basipterygoid processes (and posterior to a potentially present 2406 intertuberal plate) is flat (0); present, the ventral floor forms a shallow depression (1); present, the 2407 ventral floor is deeply excavated (2) (Ezcurra 2016: Fig. 27), ORDERED. 
2408 The pharyngeal recess was originally identified in archosauriforms but has subsequently also been

2409 described for certain non-archosauriform archosauromorphs (e.g., Azendohsaurus madagaskarensis,

2410 Flynn et al. 2010; Mesosuchus browni, Sobral \& Müller 2019). Observation of this character in the

2411 sampled taxa indicates that it can occur in two states when present. The pharyngeal recess was first

2412 described as a very deep ventral cavity (e.g., the basisphenoid recess of Witmer 1997). This occurs in

2413 Tanystropheus hydroides (PIMUZ T 2790) and Erythrosuchus africanus (BP/1/3893) among the sampled

2414 taxa. However, a much shallower excavation of the ventral surface of the parabasisphenoid posterior to

2415 the basipterygoid processes occurs in the majority of non-archosauriform archosauromorphs, as well as

2416 Youngina capensis (Gardner et al. 2010). This shallow excavation was identified as the pharyngeal recess

2417 by Sobral et al. (2016) and Sobral \& Müller (2019). We here distinguish the shallow excavation and the

2418 deeper excavation as separate character states for the first time and consider the former to likely

2419 represent an intermediate morphology between the absence of a pharyngeal recess and the deeply

2420 excavated pharyngeal recess.

2421 119) Ezcurra (2016) ch. 238 (formulation of the inapplicability criterion is slightly modified).

2422 Parasphenoid/parabasisphenoid, semilunar depression on the posterolateral surface of the bone:

2423 absent (0); present (1) (Ezcurra 2016: Fig. 28). This character is inapplicable in taxa in which the

2424 posterodorsal portion of the parasphenoid/parabasisphenoid is not ossified, resulting in an unossified

2425 gap between this element and the prootic.

2426 120) Ezcurra (2016) ch. 235 and Nesbitt et al. (2015) ch. 208 (description of state 1 slightly

2427 reformulated). Basisphenoid/parabasisphenoid, orientation of the body between the posterior end of

2428 the bone and the basipterygoid processes: horizontal (0); oblique, main axis posterodorsally-to-

2429 anteroventrally orientated (1) (Ezcurra 2016: Figs. 27 and 28).

2430 121) Ezcurra (2016) ch. $225 . \quad$ Basioccipital-parasphenoid/parabasisphenoid, contact with each other in

2431 skeletally mature individuals: loose, overlapping suture (0); tightly sutured, sometimes by an

2432 interdigitated suture, or both bones fused to each other (1) (Ezcurra 2016: Fig. 28).

2433 122) New. Basioccipital-parasphenoid/parabasisphenoid, two pneumatic foramina between the

2434 basioccipital and parabasisphenoid: absent (0); present (1) (Sobral \& Müller 2019: Figs. 3 and 13).

2435 Pneumatic foramina were described as present in several early archosauromorphs by Sobral \& Müller

2436 (2019). This character is now implemented in a quantitative phylogenetic analysis for non-

2437 archosauriform archosauromorphs for the first time.

Peer) reviewing PDF | (2020:08:51657:1:1:NEW 28 Feb 2021) 
2438 123) Ezcurra (2016) ch. 226. Basioccipital-parasphenoid/parabasisphenoid, basal tubera: absent (0); 2439 present (1) (Ezcurra 2016: Fig. 27).

2440 124) Modified from Ezcurra (2016) ch. 227. Basioccipital-parasphenoid/parabasisphenoid, low ridge 2441 between basal tubera: absent or very strongly reduced (0); present (1) (Fig. 17; Ezcurra 2016: Fig. 27).

2442 This character is scored as inapplicable in taxa that lack basal tubera.

2443 Character 227 in Ezcurra (2016) is applicable to a wide range of archosauromorphs. This character was 2444 modified to more specifically address the variation observed in the taxa sampled here. A clear but low, 2445 transversely orientated ridge is present between the basal tubera of the basioccipital of Tanystropheus hydroides (PIMUZ T 2790) and Tanystropheus longobardicus (PIMUZ T 2484). Such a ridge cannot be 2447 observed in any of the other sampled taxa.

125) Pritchard et al. (2018) ch. 318. Basioccipital, ventral margin: prominent embayment or ridge between basal tubera at least as transversely broad as occipital condyle (0); transversely narrow embayment or ridge between basal tubera, narrower than occipital condyle (1). This character is scored as inapplicable in taxa that lack basal tubera.

See the description of character 318 in Pritchard et al. (2018). An inapplicability criterion has been added to this character.

126) Ezcurra (2016) ch. 229. Basioccipital, articular surface of the occipital condyle: concave (0); hemispherical (1) (Ezcurra 2016: Fig. 28).

127) Ezcurra (2016) ch. 211 and Pritchard et al. (2015) ch 62. Otoccipital, fusion between opisthotic and exoccipital: absent or partial (0); present (1) (Ezcurra 2016: Fig. 27).

128) New, combination of character 209 of Ezcurra (2016) (=character 60 of Pritchard et al. 2015) and character 219 of Ezcurra (2016) (=character 59 of Pritchard et al. 2015).Exoccipital, morphology of the dorsal end: exoccipital columnar through dorsoventral height, forming transversely narrow dorsal contact with more dorsal occipital elements (0); dorsal portion of exoccipital exhibits dorsomedially inclined process that forms transversely broad contact with more dorsal occipital elements but exoccipitals do not meet on the dorsal margin of the foramen magnum (1); dorsal portion of exoccipital exhibits dorsomedially inclined process that meets the process of the opposite exoccipital on the dorsal margin of the foramen magnum, thus excluding the supraoccipital from contributing to the margin of the 
2466 foramen magnum (2), ORDERED (Fig. 17). This character is inapplicable in taxa without a discernable

2467 suture between the supraoccipital and the exoccipital or taxa with a fused opisthotic-exoccipital.

2468 These two characters were fused because the exclusion of the supraoccipital from the margin of the

2469 foramen magnum implies that the exoccipitals connect to each other dorsally, which is caused by an

2470 extensive dorsomedial inclination of the dorsal portions of the exoccipitals.

2471 129) Modified from Ezcurra (2016) ch. 221. Exoccipital, medial margin of their distal ends: no contact 2472 with its counterpart (0); contact with its counterpart to partially or fully exclude the basioccipital from 2473 the floor of the endocranial cavity (1) (Ezcurra 2016: Fig. 27).

2474 States 1 and 2 of character 221 in Ezcurra (2016) were fused here, because it is very difficult to 2475 distinguish between them in the sampled taxa.

2476 130) Ezcurra (2016) ch. 213 (both states slightly reformulated). Opisthotic, paroccipital processes 2477 orientation: extend laterally or slightly posterolaterally (0); deflected strongly posterolaterally at an 2478 angle of more than 20 degrees from the transverse plane of the skull (1) (Ezcurra 2016: Fig. 16).

2479 131) Pritchard et al. (2015) ch. 58. Opisthotic, paroccipital process: ends freely (0); contacts the 2480 suspensorium (1).

2481 132) Ezcurra (2016) ch. 216. Opisthotic, fossa immediately lateral to the foramen magnum: absent 2482 (0); present (1).

2483 133) Modified from Ezcurra (2016) ch. 217. Opisthotic, ventral ramus shape: pyramidal, with a 2484 tapering distal end (0); club-shaped with a large bulbous distal head (1); columnar-like shaft of the ramus 2485 and an anteroposteriorly expanded but not a bulbous distal head (2); anteroposteriorly flattened shaft of 2486 the ramus, forming a blade-like ramus in lateral view and an anteroposteriorly expanded but not a 2487 bulbous distal head (3) (Fig. 18; Ezcurra 2016: Fig. 28).

2488 This character was modified to more precisely fit the morphology of the ventral ramus of the opisthotic 2489 as we observed it for the sampled taxa.

2490 134) Ezcurra (2016) ch. 218 (state 1 slightly reformulated). Opisthotic, ventral ramus: extends 2491 further laterally than the lateralmost edge of the exoccipital in posterior view (0); ventral ramus 2492 completely or almost completely covered by the lateralmost edge of the exoccipital in posterior view (1) 2493 (Ezcurra 2016: Fig. 27). 
2494 135) Ezcurra (2016) ch. 223. Pseudolagenar recess, opening externally between the ventral surface of 2495 the ventral ramus of the opisthotic and the basal tubera: present (0); absent (1) (Ezcurra 2016: Fig. 27).

2496 136) Modified from Ezcurra (2016) ch. 19. Posttemporal fenestra, size: large, roughly similar in size 2497 to the supraoccipital (0); strongly reduced in size and much smaller than the supraoccipital (1); absent or 2498 developed as a foramen or very narrow slit (2) ORDERED (Ezcurra 2016: Fig. 27).

2499 This character has been modified according to observations of the sampled taxa. In most taxa, the 2500 posttemporal fenestra is large with little variation in its construction. However, in Azendohsaurus 2501 madagkarensis the parietal encloses the fenestra laterally, distinctly reducing it in size (Flynn et al. 2502 2010). In Erythrosuchus africanus (BP/1/4680), Proterosuchus fergusi (SAM-PK-K10603), and 2503 Proterosuchus alexanderi (NM QR 1484) the fenestra is only represented by a very narrow slit or 2504 foramen.

2505 137) Ezcurra (2016) ch. 254 (reformulated). Prootic, a clear crest on the lateral surface that is roughly 2506 orientated posterodorsally to anteroventrally (=crista prootica): absent (0); present (1) (Fig. 18; Ezcurra 2507 2016: Fig. 28).

2508 138) New. Prootic, a clear crest along the lateral surface that curves dorsally at the anterior margin 2509 of the prootic (=crista alaris): absent (0); present (1) (Fig. 18).

2510 Character 254 in Ezcurra (2016) addressed the presence of crista prootica in a phylogenetic context. 2511 However, the presence of another crest on the lateral surface of the prootic, the crista alaris, is also 2512 variable for the sampled taxa, and this is addressed with this newly formulated character for the first 2513 time.

2514 139) Pritchard et al. (2015) ch. 75 (reformulated). Prootic, paroccipital contribution: prootic does 2515 not contribute to anterior surface of paroccipital process (0); prootic contributes laterally tapering 2516 lamina to the anterior surface of the paroccipital process (1) (Fig. 18).

2517 140) Modified from Ezcurra (2016) ch. 258 and Pritchard et al. (2015) ch. $72 . \quad$ Laterosphenoid, 2518 ossification: absent (0); present, laterosphenoid is a narrow dorsoventrally orientated bone and lacks an 2519 anterior portion (1); present, laterosphenoid with an anterior portion located along the ventral surface of 2520 the parietal and frontals (2) (Fig. 18; Ezcurra: Fig. 28), ORDERED.

2521 The presence of a laterosphenoid was until recently not known for non-archosauriform 2522 archosauromorphs. However, a laterosphenoid has now also been identified in Azendohsaurus 
2523

2524

2525

2526

2527

2528

2529

2530

2531

2532

2533

2534

2535

2536

2537

2538

2539

2540

2541

2542

2543

2544

2545

2546

2547

2548

2549

madagskarensis (Flynn et al. 2010) and Tanystropheus hydroides (Spiekman et al. 2020a; Spiekman et al. 2020b). In these taxa, the laterosphenoid is small and does not extend far anteriorly as in archosauriforms. This information is added to the character. The small, unexpanded laterosphenoid is considered to represent an intermediate step between the absence of a laterosphenoid and the larger, further anterior reaching laterosphenoid of archosauriforms, and therefore the character has been ordered.

141) Ezcurra (2016) ch. 296. Stapes, shape: robust, with thick shaft (0); slender, rod-like shaft (1).

142) Ezcurra (2016) ch. 297 and Pritchard et al. (2015) ch. 77. Stapes, stapedial foramen piercing the columellar process: present (0); absent (1).

143) Simões et al. (2018) ch. $176 . \quad$ Splenial: present (0); absent (1).

144) Modified from Ezcurra (2016) ch. 266. Dentary, height at the third alveolus of the bone (or directly posterior to the tapering anterior end of the dentary in taxa with an anteriorly edentulous dentary) versus length of the alveolar margin (including edentulous anterior end if present): 0.02-0.04 (0); 0.06-0.11 (1): 0.15-0.24 (2); 0.27-0.29 (3), ORDERED RATIO (Ezcurra 2016: Figs. 17 and 18).Instead of comparing the length of the alveolar margin of the dentary to the minimum height of the dentary, it was here considered to compare it to the height of the dentary at the third alveolus, as this represents a more consistent measurement across the sampled taxa.

145) Ezcurra (2016) ch. 267 (reformulated). Dentary, shape of the tooth bearing portion (including edentulous anterior end if present): roughly straight (0); dorsally curved for all or most of its anteroposterior length (1); ventrally curved or deflected at its anterior end (2) (Ezcurra 2016: Figs. 17 and 29).

146) New. Dentary, distinct dorsoventral expansion forming a keel at the anterior end of the dentary: absent (0); present (1) (Fig. 19).

State 1 represents an autapomorphy for Tanystropheus hydroides (PIMUZ T 2790) among the sampled taxa.

147) Ezcurra (2016) ch. 270. Dentary, position of the Meckelian groove on the anterior half of the bone: dorsoventral centre of the dentary (0); restricted to the ventral border (1) (Nesbitt 2011: Fig. 27). 
2550 148) Ezcurra (2016) ch. 272. Dentary, posterodorsal process, in which its dorsal margin is confluent 2551 with the dorsal margin of the lower jaw: absent (0); present (1) (Ezcurra 2016: Figs. 17 and 29).

2552 149) Ezcurra (2016) ch. 273. Dentary, posterocentral process, in which its margins are not confluent 2553 with the dorsal or ventral margin of the lower jaw: absent (0); present (1) (Ezcurra 2016: Figs. 17 and 2554 29).

2555 150) Modified from Ezcurra (2016) ch. $275 . \quad$ Dentary, posteroventral process, in which its ventral 2556 margin is confluent with the ventral margin of the lower jaw: absent (0); present (1) (Ezcurra 2016: Figs. 255717 and 29).

2558 Character state 2 of character 275 in Ezcurra (2016) was omitted here because most of the included taxa 2559 here do not bear an external mandibular fenestra.

2560 151) Ezcurra (2016) ch. 276. Dentary, posteroventral process length: extended posteriorly to the level 2561 of the posterodorsal and/or posterocentral processes (0); extended posteriorly beyond the level of the 2562 posterodorsal and/or posterocentral processes (1) (Ezcurra 2016: Fig. 29). This character is inapplicable 2563 in taxa that lack a posteroventral process in the dentary.

2564 152) Ezcurra (2016) ch. 262 and Pritchard et al. (2015) ch. $84 . \quad$ Lower jaw, external mandibular fenestra: 2565 absent (0); present (1) (Ezcurra 2016: Figs. 17 and 29).

2566 153) New, combination of ch. 261 (partially) of Ezcurra (2016) and character 319 of Pritchard et al.

2567 (2018). Lower jaw, distinct dorsal process behind the alveolar margin (=coronoid process): absent, with 2568 only a slightly convex dorsal margin present behind the alveolar portion (0); present but low, not 2569 protruding dorsally behind the anterior process of the jugal (1); present and tall, protruding dorsally 2570 behind the anterior process of the jugal (2) (Ezcurra 2016: Fig. 29), ORDERED.

2571 Both characters 261 of Ezcurra (2016) and 319 of Pritchard et al. (2018) were considered to be 2572 informative but strongly related to each other and they were therefore combined here. It is not 2573 considered which bone forms the coronoid process because this often is hard to establish confidently in 2574 the sampled taxa. Furthermore, this information is strongly interdependent with the subsequent 2575 character (154).

2576 154) New. Separate coronoid bone: present (0); absent (1). 
2577 Although it has been previously established that several archosauromorphs lack a separate coronoid 2578 bone, this has not been coded as a character in phylogenetic analyses until now.

2579 155) Modified from Ezcurra (2016) ch. 286. Surangular, lateral shelf: absent (0); present, low ridge 2580 near dorsal margin (1); present, laterally or ventrolaterally projecting shelf with a lateral edge (2)

2581 (Ezcurra 2016: Figs. 18 and 29).

2582 States 2 and 3 of character 286 in Ezcurra (2016) were combined here because this distinction was 2583 considered to be somewhat subjective and not of relevance for the sampled taxa.

2584 156) Ezcurra (2016) ch. 288 and Pritchard et al. (2015) ch. 80 . Surangular, anterior surangular foramen 2585 on the lateral surface of the bone, near surangular-dentary contact: absent (0); present (1) (Ezcurra 2586 2016: Fig. 29).

2587 157) Ezcurra (2016) ch. 289 and Pritchard et al. (2015) ch. 81. Surangular, posterior surangular 2588 foramen on the lateral surface of the bone, positioned directly anterolateral to the glenoid fossa: absent 2589 (0); present (1) (Ezcurra 2016: Fig. 29).

2590 158) Modified from Ezcurra (2016) ch. 282. Surangular-angular, suture along the anterior half of the 2591 bones in lateral view: anteroposteriorly convex ventrally (0); roughly straight (1); anteroposteriorly 2592 concave ventrally (2) (Fig. 19; Ezcurra 2016: Fig. 29) ORDERED.

2593 In Tanystropheus hydroides (PIMUZ T 2790), Trilophosaurus buettneri (Spielmann et al. 2008), and 2594 Orovenator mayorum (Ford and Benson 2018) the surangular-angular suture is neither convex nor 2595 concave but straight, which was therefore included as a separate character state here. A straight suture 2596 is considered an intermediate step in a transformational series from concave to convex and the 2597 character has therefore been ordered.

2598 159) Modified from Ezcurra (2016) ch. 290 and Pritchard et al. (2015) ch. 82 . Angular, dorsoventral 2599 exposure on the lateral surface of the lower jaw: wide (0); forming about half of the dorsoventral height 2600 of the mandible at its greatest width (1); narrow (2) (Fig. 19; Ezcurra 2016: Fig. 29) ORDERED.

2601 In Tanystropheus hydroides (PIMUZ T 2790), Tanystropheus longobardicus (PIMUZ T 2484), 2602 Azendohsaurus madagaskarensis (Flynn et al. 2011), Proterosuchus fergusi (SAM-PK-11208), and 2603 Proterosuchus alexanderi (NM QR 1484) the angular covers approximately half of the lateral surface of 2604 the mandible posteriorly, which was therefore included as separate character state here. This exposure 
2605 is considered an intermediate step in a transformational series from a very wide to a very narrow 2606 exposure and the character has therefore been ordered.

2607 160) Pritchard et al. (2015) ch. 83 (state 0 reformulated). Angular, exposure on lateral mandibular 2608 surface: terminates distinctly anterior to the glenoid (0); extends to the glenoid (1).

2609 161) Modified from Senter (2004) ch. 16 Location of glenoid fossa compared to tooth row of the 2610 dentary: roughly at the same dorsoventral level as the tooth row (0); considerably ventrally displaced 2611 compared to the tooth row (1) (Fig. 19).

2612 In several archosauromorphs (Tanystropheus hydroides, PIMUZ T 2790; Tanystropheus longobardicus, 2613 PIMUZ T 2482; Tanytrachelos ahynis, YPM 7496a; Pectodens zhenyuensis, IVPP V18578;

2614 Dinocephalosaurus orientalis, IVPP V13767; and Azendohsaurus madagaskarensis, Flynn et al. 2011) and 2615 in Gephyrosaurus bridensis (Evans 1980), the glenoid fossa is located distinctly ventrally compared to the 2616 dentary tooth row. This character was first employed by Senter (2004).

2617 162) Ezcurra (2016) ch. 283. Articular, retroarticular process: absent (0); anteroposteriorly short, 2618 being poorly developed posteriorly to the glenoid fossa (1); anteroposteriorly long, extending 2619 considerably posterior to the glenoid fossa (2) ORDERED (Ezcurra 2016: Figs. 17 and 29).

2620 163) Ezcurra (2016) ch. $284 . \quad$ Articular, retroarticular process: not upturned (0); upturned (1) (Ezcurra 2621 2016: Figs. 17 and 29). This character is scored as inapplicable in taxa that lack a retroarticular process.

2622 164) Pritchard et al. (2015) ch. $92 . \quad$ Marginal dentition, arrangement: single row of marginal teeth

2623 (0); multiple zahnreihen in maxilla and dentary (1).

2624 Characters 73 and 279 in Ezcurra (2016) treat the number of tooth rows on the upper and lower jaws 2625 separately. We consider these characters to be strongly interdependent for the sampled taxa and 2626 therefore prefer to treat both jaws for this feature in one character here.

2627 165) New. Marginal dentition, anterior teeth are interlocking fangs forming a fish-trap sensu 2628 Rieppel (2002): absent (0); present (1) (Fig. 19). This character is inapplicable in taxa with an edentulous 2629 premaxilla.

2630 In Tanystropheus hydroides (PIMUZ T 2790), Tanystropheus longobardicus (MSNM BES SC 1018), and 2631 Dinocephalosaurus orientalis (IVPP V13767) the anterior marginal dentition is fang-like and elongate. 2632 These teeth interlock to form a 'fish-trap' type dentition. 
2633 166) Modified from Ezcurra (2016) ch. 280. Marginal dentition, occlusion of marginal teeth: single-

2634 sided overlap (excluding potentially present interlocking fish-trap dentition anteriorly) (0); flat occlusion

2635 (1); teeth interlocking tightly (2) (Ezcurra 2016: Fig. 14). This character is inapplicable in taxa in which

2636 multiple tooth rows are present on the marginal dentition.

2637 The character states were modified to more specifically address the morphologies observed in the 2638 sampled taxa.

2639 167) Ezcurra (2016) ch. 298. Marginal dentition, posterior extent of mandibular and maxillary tooth 2640 rows: subequal (0); maxillary teeth extending further posteriorly (1).

2641 168) Ezcurra (2016) ch. 277. Marginal dentition, posteriormost dentary teeth: on the anterior half of 2642 lower jaw (0); on the posterior half of lower jaw (1) (Ezcurra 2016: Fig. 17).

2643 169) Ezcurra (2016) ch 299. Marginal dentition, tooth implantation: subthecodont (=protothecodont)

2644 (0); ankylothecodont (teeth fused to the bone at the base of the crown by bony ridges and the root can 2645 be discerned; there is continuous tooth replacement) (1); pleurodont (2); acrodont (teeth fused to the 2646 bone in adults so that no root can be discerned) (3); thecodont (4) (Ezcurra 2016: Figs. 12, 14 and 22).

2647 170) Ezcurra (2016) ch. 308. Marginal dentition, multiple maxillary and dentary tooth crowns 2648 distinctly mesiodistally expanded above the root: absent (0); present (1) (Ezcurra 2016: Fig. 14).

2649 171) Modified from Ezcurra (2016) ch. 303. Marginal dentition, maxillary teeth: straight or very 2650 slightly recurved (0); distinctly recurved (1) (Ezcurra 2016: Fig. 14). This character is not applicable in taxa 2651 with maxillary teeth that expand above the root or that possess multiple tooth rows in the maxilla.

2652 Certain taxa have very slightly recurved teeth (e.g., Petrolacosaurus kansensis, Czatkowiella harae, and 2653 Orovenator mayorum). However, we choose not to maintain a separate character state for this 2654 morphology as in these taxa not all teeth are recurved and many are straight, therefore forming a very 2655 minimal distinction from the straight morphology. Only taxa in which the curvature of the teeth is 2656 distinct are scored as 1.

2657 172) Ezcurra (2016) ch. 304. Marginal dentition, serrations on the maxillary/dentary crowns: absent 2658 (0); distinctly present on the distal margin and usually apically restricted, low or absent on the mesial 2659 margin (1); present and distinct on both margins (2) (Ezcurra 2016: Fig. 14). 
2660 173) Ezcurra (2016) ch. 306. Marginal dentition, multiple maxillary or dentary tooth crowns with 2661 longitudinal labial or lingual striations or grooves: absent (0); present (1) (Ezcurra 2016: Fig. 14).

2662 174) Modified from Pritchard et al. (2015) ch. $98 . \quad$ Marginal dentition, tooth shape at crown base of 2663 the maxillary teeth: circular (0); labiolingually compressed (1); labiolingually wider than mesiodistally 2664 long (2) (Fig. 20).

2665 This character is only scored for the maxillary teeth because certain taxa exhibit a heterodont dentition, 2666 for instance in the form of large fang-like teeth on the premaxilla and anterior portion of the dentary 2667 (Tanystropheus hydroides, PIMUZ T 2790; and Dinocephalosaurus orientalis, IVPP V13767). The presence 2668 of marginal teeth that are oval in cross-section has widely been considered an important character that 2669 is typically diagnostic for Archosauriformes (e.g., Dilkes 1998, Jalil 1997, Pritchard et al. 2015), since it 2670 occurs widely in archosauriforms and only rarely in non-archosauriform diapsids (Ezcurra 2016).

2671 However, we found that the condition in which marginal teeth are labiolingually narrower than 2672 mesiodistally wide is more widespread than previously considered, occurring, among others, in 2673 Tanystropheus hydroides (PIMUZ T 2790), Tanystropheus longobardicus (PIMUZ T 3901), Macrocnemus 2674 bassanii (PIMUZ T 2477), Macrocnemus fuyuanensis (PIMUZ T 1559), Langobardisaurus pandolfii (MFSN 2675 1921), and Dinocephalosaurus orientalis (IVPP V13767). This morphology is distinct from the virtually 2676 circular cross-sections of teeth present in for instance Czatkowiella harae (ZPAL RV/100, figure 4 of 2677 Borsuk-Białynicka \& Evans 2009b).175) Modified from Pritchard et al. (2015) ch. $93 . \quad$ Marginal 2678 dentition, morphology of crown base: all tooth crowns form a single, pointed or rounded crown (0); at 2679 least some tooth crowns form a flattened platform with pointed cusps (1); at least some tooth crowns 2680 have three, mesiodistally arranged cusps (2).

2681 The character states were modified to more specifically address the morphologies observed in the 2682 sampled taxa.

2683 176) Ezcurra (2016) ch. 310. Cervical, dorsal, sacral and caudal vertebrae, notochordal canal piercing 2684 the centrum: present throughout ontogeny (0); absent in adults (1) (Ezcurra 2016: Fig. 31).

2685 177) Ezcurra (2016) ch. 313. Presacral vertebrae, at least one or more cervical or anterior dorsal with 2686 parallelogram-shaped centra in lateral view, in which the anterior articular surface is situated higher 2687 than the posterior one: absent (0); present (1) (Ezcurra 2016: Figs. 11 and 33).

2688 178) New. Cervical vertebrae, maximum height of postaxial anterior or middle cervical neural 2689 spines: considerably taller than the posterior articular surface of the centrum (0); approximately equally 
2690 tall as the posterior articular surface of the centrum (1); considerably shorter than the posterior articular 2691 surface of the centrum (2); low neural spines are only present at the anterior and posterior ends of the 2692 vertebrae but are completely or virtually lost at their anteroposterior midpoints (3); neural spine is 2693 completely reduced or lost (4) (Fig. 21; Ezcurra 2016: Fig. 11), ORDERED.

2694 Characters 342 and 344 in Ezcurra (2016) addressed the height of the neural spine in the postaxial 2695 cervical vertebrae, which is a variable and phylogenetically important trait among tanystropheids. We 2696 have combined the information of these two characters, because we considered them to be 2697 interdependent, and modified the states distinctly to address the specific morphologies observed in the 2698 sampled taxa.

2699 179) New. Cervical vertebrae, shape of distal margin of anterior and middle cervical postaxial 2700 neural spines in lateral view: slightly convex (0); completely straight along anteroposterior length (1); 2701 concave (2) (Fig. 22). This character is inapplicable in taxa that have reduced the neural spine of their 2702 anterior and mid cervicals completely or at their anteroposterior midpoint.

2703 We find that in several taxa the distal margin of the neural spine of the anterior to mid cervical 2704 vertebrae is completely straight along its entire anteroposterior length (e.g., Macrocnemus bassanii, 2705 PIMUZ T 4822; and Pamelaria dolichotrachela, ISIR 316/1). This straight margin often, but not always, 2706 occurs together with a distally expanded neural spine (=spine table). However, due to both structures 2707 also occurring without the presence of the other, they were scored here as separate characters.

2708 Furthermore, the distal margin of the neural spines of certain taxa are conspicuously concave 2709 (particularly in Dinocephalosaurus orientalis, Rieppel et al. 2008).

2710 180) New, combination of Ezcurra (2016) ch. 320 and 321 . Cervical vertebrae, distal expansion of 2711 the anterior to middle postaxial cervical neural spines (not mammillary process): absent (0); present, 2712 gradual transverse expansion of the distal half of the neural spine (1); present, but transverse expansion 2713 is restricted to the distal end of the neural spine (=spine table) (2) (Fig. 22). This character is inapplicable 2714 in taxa that have reduced the neural spine of their anterior and mid cervicals completely.

2715 A distal expansion of the postaxial neural spines was previously addressed by character 117 in Pritchard 2716 et al. (2015) and characters 320 and 321 in Ezcurra (2016). Here, we combined information of these 2717 characters to form a new character that addresses the variation seen in this trait in the sampled taxa. 2718 We consider the gradual transverse expansion to represent a separate state from the presence of a 2719 spine table, following Ezcurra (2016). However, since a gradual expansion and a distinct spine table both 
2720 address a widening of the neural spine, which is separate from the presence of mammillary processes, 2721 we consider them part of the same morphological character. This character should only be scored in 2722 skeletally mature specimens since a transverse expansion of the neural spine is generally absent in early 2723 ontogenetic stages.

2724 181) Modified from Simões et al. (2018) ch. $228 . \quad$ Presacral vertebrae, type of articular surface: 2725 opisthocoelous (0); procoelous (1); amphicoelous (2); acoelous (3). This character is inapplicable in taxa 2726 that have a notochordal canal running through their centra.

2727 The articulation surfaces of the centra of presacral vertebrae was previously considered by characters 2728101 and 102 in Pritchard et al. (2015), which considered the anterior and posterior surfaces separately. 2729 We follow Simões et al. (2018) and treat the articulation surfaces of the centra as a single character.

2730 182) Nesbitt (2011) ch. 177 . Presacral vertebrae, postaxial intercentra: present (0); absent (1).

2731 The presence of intercentra was scored separately for postaxial cervical vertebrae and dorsal vertebra in 2732 characters 346 and 366 in Ezcurra (2016). However, we score only the presence or absence of postaxial 2733 intercentra since in most cases for the sampled taxa the presence of intercentra often occurs in both 2734 segments of the vertebral column. Therefore, separating these segments results in overscoring of the 2735 presence of postaxial intercentra.

2736 183) Ezcurra (2016) ch. 326 and Nesbitt et al. (2015) ch. $243 . \quad$ Cervical vertebrae, centrum of atlas in 2737 skeletally mature individuals: separate from axial intercentrum (0); fused to axial intercentrum (1) 2738 (Ezcurra 2016: Fig. 30).

2739 184) New. $\quad$ Cervical vertebrae, proatlas elements dorsal to atlantal neural arches: present (0); 2740 absent or fused with atlantal neural arch (1) (Fig. 23).

2741 No proatlases are present in Tanystropheus hydroides (PIMUZ T 2790), in contrast to all other sampled 2742 taxa for which this character could be scored.

2743 185) Modified from Ezcurra (2016) ch. 328. Cervical vertebrae, height of neural spine of the axis: 2744 ratio between the maximum height of the neural spine and the posterior articular surface height of the 2745 centrum of the axis: 0.46-0.59 (0); 0.76-1.21 (1): 1.33-1.66 (2); 2.06-2.23 (3), ORDERED RATIO (Ezcurra 2746 2016: Fig. 30). 
2747 The distinction between the states of character 328 of Ezcurra (2016) is considered to be ambiguous for

2748 the taxa sampled here. Therefore, the states were modified into ratios to make a more discrete

2749 distinction between the states.

2750 186) Ezcurra (2016) ch. 329 (slightly reformulated). Cervical vertebrae, shape of the neural spine of

2751 the axis in lateral view: expanded posterodorsally or the height of the anterior portion is equivalent to

2752 the posterior height (0); expanded anterodorsally (1) (Ezcurra 2016: Fig. 30).

2753 187) Ezcurra (2016) ch. 331. Cervical vertebrae, lengths of the fourth or fifth cervical centra versus the

2754 heights of their anterior articular surfaces: 0.63-5.06 (0); 6.33-12.12 (1); 14.58-15.58 (2); $17.08-18.67$ (3);

2755 20.05-20.51 (4), ORDERED RATIO (Ezcurra 2016: Fig. 15).188) Ezcurra (2016) ch. $332 . \quad$ Cervical

2756 vertebrae, diapophysis and parapophysis of anterior to middle cervical postaxial vertebrae: single facet

2757 or both situated on the same process (0); situated on different processes and well-separated (1); situated

2758 on different processes and nearly touching (2) (Ezcurra 2016: Fig. 30).

2759 189) Modified from Ezcurra (2016) ch. 340. Cervical vertebrae, laminae extending posteriorly from

2760 the base of the dia-and/or parapophysis in anterior and middle postaxial cervical vertebrae: absent (0);

2761 present (1) (Ezcurra 2016: Fig. 30).

2762 Laminae project from the base of the dia -and/or parapophysis in most of the sampled taxa, except for

2763 Youngina capensis (BP/1/3859), Erythrosuchus africanus (BP/1/5207), Mesosuchus browni (SAM-PK-

2764 5882), and Planocephalosaurus robinsonae (Fraser \& Walkden 1984).

2765 190) Ezcurra (2016) ch. 336. Cervical vertebrae, epipophysis in postaxial cervicals: absent (0); present

2766 in at least the third to fifth cervical vertebrae (1) (Ezcurra 2016: Figs. 30 and 33).

2767 191) Modified from Pritchard et al. (2018) ch. 271. Cervical vertebrae, posterior extension of

2768 epipophysis: not extended posterior to the postzygapophysis (0); overhanging the postzygapophysis

2769 posteriorly (1) (Fig. 24). This character is inapplicable in taxa that lack epipophyses on their cervical

2770 vertebrae.

2771 In all tanystropheids except for certain specimens of Tanystropheus "conspicuus" (U-MO BT 740),

2772 Sclerostropheus fossai (MCSNB 4035), Macrocnemus fuyuanensis (IVPP V15001), and Langobardisaurus

2773 pandolfii (MCSNB 2883) the epipophyses are well-developed and extend posteriorly beyond the level of

2774 the postzygapophyses. In all other sampled taxa that bear epipophyses, they are not extended as far

2775 posteriorly. The character was modified from character 271 of Pritchard et al. (2018) because the

Peer) reviewing PDF | (2020:08:51657:1:1:NEW 28 Feb 2021) 
2776 distinction between states 1 and 2 therein was considered to be difficult to distinguish confidently in the 2777 sampled taxa.

2778 192) Modified from Ezcurra (2016) ch. 338 and Nesbitt et al. (2015) ch. $213 . \quad$ Cervical vertebrae, 2779 anterior cervical vertebrae (presacral vertebrae 3-5) postzygapophyses: postzygapophyseal trough 2780 (sensu Rieppel 2001) formed by a well-developed posteriorly extending shelf (=transpostzygapophyseal 2781 lamina) that in some cases bears a notch on its posterior end: absent (0); present (1) (Fig. 25).

2782 This character was modified based on detailed observations of the vertebrae of Tanystropheus spp.

2783 193) Pritchard et al. (2015) ch. $113 . \quad$ Cervical vertebrae, neural spine base of anterior postaxial 2784 cervical vertebrae: anteroposteriorly elongate, subequal in length to the neural arch (0);

2785 anteroposteriorly shortened, spine restricted to posterior half of neural arch (1). This character is 2786 inapplicable in taxa that have completely reduced the neural spine of their anterior and mid cervicals.

2787 The inapplicability criterion was added and the formulation of the character has been modified slightly. 194) New, combination of Ezcurra (2016) ch. 343 and Pritchard et al. (2015) ch. $116 . \quad$ Cervical vertebrae, orientation of the anterior margin of the neural spine of anterior and middle postaxial cervical vertebrae: straight or posterodorsally inclined (0); anterodorsally inclined at an angle of more than 60 degrees from the horizontal plane (1); anterodorsally inclined at an angle of less than 60 degrees from the horizontal plane (2) (Ezcurra 2016: Figs. 30 and 33), ORDERED. This character is inapplicable in taxa that have completely reduced the neural spine of their anterior and mid cervicals.

2794 The notch referred to in character 115 of Pritchard et al. (2015) was reinterpreted as an anterior overhang or inclination in character 343 of Ezcurra (2016). Here, this inclination is considered to represent a similar morphology as the inclination described by character 116 of Pritchard et al. (2015) and therefore these characters were fused here. The degree of an anterodorsal inclination of the anterior margin of the neural spine in the anterior to middle postaxial cervical vertebrae is strongly variable among the sampled taxa, and therefore we distinguish between two clearly demarcated states. State 1 represents an intermediate morphology between states 0 and 2, and the character was therefore ordered. This character is scored as ? for Tanystropheus hydroides, Tanystropheus longobardicus, and Tanystropheus "conspicuus". In these taxa the anterior margin of the neural spine is complex as it is bifurcated and therefore does not allow for a confident scoring of this character (see fig. 57 of Nosotti 2007). 
2805

2806

2807

2808

2809

2810

2811

2812

2813

2814

2815

2816

2817

2818

2819

2820

2821

2822

2823

2824

2825

2826

2827

2828

2829

2830

2831

2832

2833

195) Modified from Ezcurra (2016) ch. $324 . \quad$ Cervical vertebrae, total number: six or fewer (0); between seven and 10 (1); between 11 and 13 (2); more than 13 (3), ORDERED.

The states were modified based on the distribution of the number of cervical vertebrae in the sampled taxa.

196) New. Cervical vertebrae, presence of a foramen on the ventral surface of the centrum around the anteroposterior midpoint: absent (0); present (1) (Fig. 26).

A conspicuous nutrient foramen (foramina venae vertebralis sensu Wild 1973) is present on the ventral surface of several cervical vertebrae of Tanystropheus "conspicuus" (Spiekman \& Scheyer 2019) and Gephyrosaurus bridensis (Evans 1981). This foramen is absent in other taxa for which this character could be assessed.

197) Pritchard et al. (2015) ch. 109 (reformulated). Cervical vertebrae, anterior to mid postaxial cervical vertebrae, shape of ventral surface in the coronal plane excluding keel: rounded or curved (0); ventral face flattened (1).

198) New. Cervical vertebrae, neural canal of anterior to mid cervical vertebrae separated from vertebral centrum (0); neural canal enters into a cavity of the vertebral centrum (1) (Spiekman et al. 2020b: Fig. 32).

In the tanystropheids Macrocnemus bassanii, Tanytrachelos ahynis, and Tanystropheus spp. the neural canal of the anterior to mid cervical vertebrae enters the vertebral centrum. This morphology was first described for Tanystropheus "conspicuus" by Edinger (1924) and has recently been identified for several other tanystropheids through micro computed tomography. Although this character has so far not be examined for most taxa, it might represent a widespread feature among tanystropheids.

199) Ezcurra (2016) ch. 349 (state 2 reformulated). Cervical ribs, shape: short, being less than two times the length of its respective vertebra, and tapering at a high angle to the neck (0); short, being less than two times the length of its respective vertebra, and shaft parallel to the neck (1); very long, at least some ribs being more than two times the length of its respective vertebra, and parallel to the neck (2) (Fig. 27; Nesbitt 2011: Figs. 28 and 30).

200) Modified from Ezcurra (2016) ch. 350 and Pritchard et al. (2015) ch. 105. Cervical ribs, anterior free-ending process (=accessory process) on anterior surface of anterior cervical ribs: absent (0); present and short, not reaching anterior to the prezygapophyses of the corresponding vertebra when in 
2834

2835

2836

2837

2838

2839

2840

2841

2842

2843

2844

2845

2846

2847

2848

2849

2850

2851

2852

2853

2854

2855

2856

2857

2858

2859

2860

2861

2862

articulation (1); present and long, extending anterior to the prezygapophyses of the corresponding vertebra when in articulation (2) (Fig. 27; Ezcurra 2016: Fig. 30), ORDERED.

The anterior free-ending process of the cervical ribs in certain non-crocopodan archosauromorphs (Czatkowiella harae, ZPAL RV/937; Sclerostropheus fossai, MCSNB 4035; Tanytrachelos ahynis, VMNH 120346a; Pectodens zhenyuensis, IVPP V18578; Dinocephalosaurus orientalis, Rieppel et al. 2008) is particularly elongate and extends distinctly anterior to the corresponding vertebra. This represents a clearly separate morphology from the shorter processes seen in most archosauromorphs, and is therefore treated as a new, separate character state. The short processes are considered an intermediate morphology in a transformational series between the absence of the process and the elongate processes, and therefore the character has been ordered.

201) Modified from Ezcurra (2016) ch. 320. Presacral vertebrae, mammillary processes (sensu Ezcurra \& Butler 2015b) occurring in the posterior cervical to mid-dorsal vertebrae: absent (0); present (1) (Ezcurra 2016: Figs. 31, 32 and 34).

We follow the description of Ezcurra \& Butler (2015b) for our identification of mammillary processes. Therefore, we differentiate mammillary processes from a transverse expansion of the neural spine (=spine table) by the presence of a longitudinal cleft between the process and the dorsal margin of the spine in the former, which results in a neural spine with three separate projections on its distal end rather than a single flattened surface, as in the latter. The presence of mammillary processes is considered to preclude the possibility of a distally expanded neural spine in the anterior to mid-dorsal vertebrae, since an expansion is already formed by the mammillary processes. This character should only be scored in skeletally mature specimens since mammillary processes are generally not yet developed in early ontogenetic stages.

202) New. Dorsal vertebrae, shape of distal margin of anterior to middle dorsal neural spines in lateral view: slightly convex in lateral view (0); completely straight along anteroposterior length in lateral view (1) (Fig. 28). This character is inapplicable in taxa that possess mammillary processes.

As in the cervical vertebrae, the anterior to mid dorsal vertebrae of certain taxa bear a straight dorsal margin of the neural spine. This character is scored separately from character 179 because several of the sampled taxa exhibit clear variation in the shape of the distal margin of the neural spine between the dorsal and cervical vertebrae. 
2863 203) New, combination of Ezcurra (2016) ch. 320 and 321, and Pritchard et al. (2015) ch. 125. Dorsal 2864 vertebrae, distal expansion of the dorsal neural spines (not mammillary process) of the anterior to mid 2865 dorsal vertebrae: absent (0); present, gradual transverse expansion of the distal half of the neural spine 2866 (1); present, but transverse expansion is restricted to the distal end of the neural spine (=spine table) (2) 2867 (Fig. 28). This character is inapplicable in taxa that bear mammillary processes on their dorsal vertebrae.

2868 This character describes the same morphology as is described for the cervical vertebrae in character 2869 180. The occurrence of the expansion in the cervical and dorsal vertebrae is split into two different 2870 characters for the same arguments as for character 202. This character should only be scored in skeletally mature specimens since a transverse expansion of the neural spine is generally absent in early ontogenetic stages.

2874

204) Modified from Senter (2004), ch. 42.

Dorsal vertebrae, total number of dorsal vertebrae: $\leq 24$

2875

2876

2877

2878

2879

2880

2881

2882

2883

2884

2885

2886

2887

2888

2889

2890

(0); $\geq 25$ (1).

The states of this character were modified to distinguish between the very high number of dorsal vertebrae seen in Dinocephalosaurus orientalis (Rieppel et al. 2008) and the 13 to 20 dorsal vertebrae seen in other taxa. No other states are incorporated because the exact number of dorsal vertebrae is hard to establish in many of the sampled taxa.

205) Ezcurra (2016) ch. 352 (reformulated). Dorsal vertebrae, length versus height of the centrum at the level of its posterior articular surface in posterior dorsals: 0.83-1.25 (0); 1.36-1.88 (1); 2.16-2.20 (2); 2.31-2.40 (3); 2.53-2.76 (4), ORDERED RATIO.206) Modified from Ezcurra (2016), ch. $354 . \quad$ Dorsal vertebrae, lateral fossa on the centrum below the neurocentral suture: absent (0); present (1) (Ezcurra 2016: Figs. 31 and 34).

State 2 of character 254 of Ezcurra (2016) is excluded because it does not apply to the sampled taxa.

207) Modified from Nesbitt (2011) ch. 199. Dorsal vertebrae, development of the transverse process in middle dorsals: short, projecting only slightly beyond the lateral surface of the neural arch (0); long (1) (Ezcurra 2016: Fig. 32).

This character was modified based on the observed morphologies in the sampled taxa.

208) Ezcurra (2016), ch. 359. Dorsal vertebrae, hyposphene-hypantrum accessory intervertebral articulation in middle-posterior dorsals: absent (0); present (1) (Ezcurra 2016: Figs. 31 and 32). 
2891 209) Ezcurra (2016) ch. 361. Dorsal vertebrae, dorsally opening pit lateral to the base of the neural 2892 spine: absent (0); shallow (fossa) (1); developed as a deep pit (2) ORDERED (Ezcurra 2016: Fig. 34).

2893 210) Ezcurra (2016) ch. $363 . \quad$ Dorsal vertebrae, fan-shaped neural spine in lateral view: absent (0); 2894 present (1).

2895 211) Modified from Pritchard et al. (2015) ch. 129. Dorsal vertebrae, height of neural spines in mid2896 dorsals: tall, greater in dorsoventral height than anteroposterior length (0); long and low, approximately similar in dorsoventral height and anteroposterior length or less in height than in length (1).

We modified the character so that it only applies to mid-dorsal vertebrae, because anterior dorsal vertebrae often have a different morphology from more posterior vertebrae, and their inclusion therefore might result in inconsistent character scoring.

212) Pritchard et al. (2015) ch. 121. Dorsal vertebrae, position of parapophysis (or ventral margin of dorsal synapophysis) in posterior dorsals: positioned partially on lateral margin of centrum (0); positioned entirely on neural arch (1).

213) Pritchard et al. (2015) ch. 122 (reformulated). Dorsal ribs, proximal end of anterior dorsal ribs: holocephalous (one facet) (0); dichocephalous (two facets) (1); tricephalous (three facets) (2).

214) Ezcurra (2016) ch. 368. Dorsal ribs, proximal end of middle dorsal ribs: dichocephalous (0); holocephalous (1). This character is inapplicable in taxa that have holocephalous anterior dorsal ribs since these imply the presence of holocephalous middle dorsal ribs.

2909 The inapplicability criterion has been added.

215) Ezcurra (2016) ch. 372 and Nesbitt et al. (2015) ch. 216. Sacral ribs, anteroposterior length of the first primordial sacral rib versus the second primordial sacral rib in dorsal view: primordial sacral rib one is longer anteroposteriorly than primordial sacral rib two (0); primordial sacral rib two is about the same length or longer anteroposteriorly than primordial sacral rib one (1).

216) Ezcurra (2016) ch. 373 and Pritchard et al. (2015) ch. 131. Sacral ribs, second rib shape: single unit (0); bifurcates distally into anterior and posterior processes (1) (Ezcurra 2016: Fig. 35). taxa without a bifurcated second sacral rib. 
2919

2920

2921

2922

2923

2924

2925

2926

2927

2928

2929

2930

2931

2932

2933

2934

2935

2936

2937

2938

2939

2940

2941

2942

2943

2944

2945

218) Ezcurra (2016) ch. 375 (reformulated). Sacral and caudal vertebrae, transverse processes/ribs of sacral and anterior caudal vertebrae in skeletally mature individuals: rib/transverse process and vertebra unfused (0); rib/transverse process and vertebra fused to each other (1) (Ezcurra 2016: Fig. 35).

219) Modified from Ezcurra (2016) ch. 377. Caudal vertebrae, length of the transverse process + rib versus length across zygapophyses in anterior caudal vertebrae (third to fifth caudal): 0.62-1.28 (0); 1.621.77 (1); 1.90-2.00 (2); 2.50-2.60 (3), ORDERED RATIO (Ezcurra 2016: Fig. 35).

This character was modified slightly to specify on which caudal vertebrae this character should be scored.

220) Modified from Dilkes (1998) ch. 88. Caudal vertebrae, height versus maximum anteroposterior length of anterior caudal neural spine (measured in one of the first five caudals): 0.420.83 (0); 1.00-1.60 (1); 2.00-2.24 (2); 2.39-2.53 (3); 2.93-3.07 (4), ORDERED RATIO.221) Modified from Pritchard et al. (2015) ch. 134. Caudal vertebrae, orientation of transverse processes: base of process perpendicular to the long axis of the vertebra or slightly posterolaterally angled (0); processes distinctly angled posterolaterally from base (1).

The states were modified to represent a clearer morphological distinction between them based on the sampled taxa.

222) Modified from Dilkes (1998) ch. 141 Chevrons, curvature of haemal spines in mid-caudal vertebrae: no curvature or posterior curvature (0); anterior curvature present (1).

The states were modified to represent a clearer morphological distinction between them based on the sampled taxa.

223) Modified from Pritchard et al. (2015) ch. 136. Chevrons, shape of haemal spine: tapers along its anteroposterior length (0); maintains breadth along its length (1); gradually broadens distally (2); broadens abruptly distally, forming an inverted T shape (3).

This character was modified based on the observed morphologies in the sampled taxa.

224) New. Chevrons, anteroposterior length of vertebral centrum versus proximodistal length of corresponding haemal spine in anterior caudals (third to fifth caudal): 0.33-0.52 (0); 0.65-0.81 (1); 1.00 1.04 (2), ORDERED RATIO (Fig. 29). 
2946 The relative length of the chevrons in the anterior caudal vertebrae differs among the sampled taxa and

2947 is possibly phylogenetically informative and it was therefore included as a character here.

2948 225) Pritchard et al. (2015) ch. 200. Heterotopic ossifications: absent in a minimum of 5 individuals

2949 (0); present (1) (Fig. 30).

2950 See the character description of character 200 in Pritchard et al. (2015).

2951 226) Ezcurra (2016) ch. 384 . Scapulocoracoid, both bones fuse with each other in skeletally mature 2952 individuals: present (0); absent (1) (Ezcurra 2016: Fig. 36).

2953 227) New, combination of ch. 385 and 388 of Ezcurra (2016). Scapulocoracoid, the anterior margin at 2954 the level of the suture between both bones: roughly continuous margin (0); distinct notch present (1); 2955 large fenestra between scapula and coracoid (scapulocoracoidal fenestra) present (2) (Fig. 31; Ezcurra 2956 2016: Fig. 36).

2957 Characters 385 and 388 in Ezcurra (2016) are fused because both refer to the anterior margin of the 2958 scapulocoracoid and the presence of state 1 precludes the possibility of state 2 , and vice versa.

2959 However, because it is not clear whether the notch and the fenestra represent transitional 2960 morphologies of the same structure, the character is not ordered.

2961 228) Modified from Pritchard et al. (2015) ch. 145 and Ezcurra (2016) ch. 389. Scapula, scapular blade 2962 dorsally or posterodorsally orientated with a rectangular outline (0); blade is largely posteriorly directed 2963 and semi-circular in outline with a continuously curved anterior/dorsal margin (1) (Fig. 31).

2964 The semi-circular or semi-lunar shape of the scapula in tanystropheids and Pectodens zhenyuensis 2965 represents a unique morphology among archosauromorphs and has been previously incorporated in 2966 phylogenetic analyses. We have redescribed this character to more specifically address this morphology 2967 as it is observed in a wide sample of taxa.

2968 229) Modified from Ezcurra (2016) ch. 390 and Nesbitt et al. (2015) ch. 219. Scapula, anterior margin 2969 of the scapular blade in lateral view, excluding the margin of a potentially present scapulocoracoidal 2970 fenestra: straight or convex along entire length (0); distinctly concave (1) (Ezcurra 2016: Fig. 36). This 2971 character is inapplicable in taxa that have a semicircular scapular blade.

2972 This character was modified to prevent it from being interdependent with characters 227 and 228. 
2973 230) Modified from Ezcurra (2016) ch. 391 and Nesbitt et al. (2015) ch. $220 . \quad$ Scapula, constriction 2974 distal to the glenoid: minimum anteroposterior length greater than half the proximodistal length of the 2975 scapula (0); minimum anteroposterior length less than half but more than a quarter of the proximodistal 2976 length of the scapula (1); minimum anteroposterior length less than a quarter of the proximodistal 2977 length of the scapula (2) (Ezcurra 2016: Figs. 36 and 37), ORDERED. This character is inapplicable in taxa 2978 that have a semi-circular scapular blade.

2979 This character was modified based on the observed morphologies in the sampled taxa. The 2980 inapplicability criterion was added because a semi-circular shape of the scapular blade implies that it is 2981 comparatively much wider than the other morphologies. Thus, scoring those scapulae for this character 2982 would always result in state 0 , thus representing an overscoring of the semi-circular shaped scapular 2983 blade.

2984 231) Ezcurra (2016) ch. $392 . \quad$ Scapula, supraglenoid foramen: absent (0); present (1).

2985 232) Ezcurra (2016) ch. 398 (state 2 reformulated). Coracoid, posterior border in lateral view: 2986 unexpanded posteriorly (0); moderately expanded posteriorly (1); strongly expanded posteriorly - the 2987 entire border, not only the posteroventral region as is the case in the postglenoid process - and, as a 2988 result, the articulated scapula and coracoid are L-shaped in lateral view (in taxa in which the scapular 2989 blade is not semi-circular in shape) (2), ORDERED (Ezcurra 2016: Fig. 37).

2990 233) Ezcurra (2016) ch. 404 and Pritchard et al. (2015) ch. 140. Cleithrum: present (0); absent (1).

2991 234) Ezcurra (2016) ch. $405 . \quad$ Interclavicle: present (0); absent (1) (Ezcurra 2016: Fig. 15).

2992 235) Modified from Ezcurra (2016) ch. 406. Interclavicle, long anterior process, resulting in a cross2993 shaped interclavicle in ventral or dorsal view: present (0); absent (1) (Ezcurra 2016: Fig. 38). This 2994 character is inapplicable in taxa that lack an ossified interclavicle.

2995 An inapplicability criterion was added.

2996 236) Ezcurra (2016) ch. 407 and Pritchard et al. (2015) ch. 143. Interclavicle, anterior margin with a 2997 median notch: absent (0); present (1) (Ezcurra 2016: Fig. 38). This character is inapplicable in taxa that 2998 lack an ossified interclavicle.

2999 An inapplicability criterion was added. 
3000 237) Ezcurra (2016) ch. 409. Interclavicle, webbed between lateral and posterior processes: present, 3001 proximal half of the bone subtriangular or diamond-shaped (0); absent, sharp angles between processes 3002 (1) (Ezcurra 2016: Fig. 38). This character is inapplicable in taxa that lack an ossified interclavicle.

3003 An inapplicability criterion was added.

3004 238) Ezcurra (2016) ch. 411 and Pritchard et al. (2015) ch. 144. Interclavicle, posterior ramus: little 3005 change in width along entire length (0); gradual transverse expansion present (1) (Ezcurra 2016: Fig. 38). 3006 This character is inapplicable in taxa that lack an ossified interclavicle.

3007 An inapplicability criterion was added.

3008 239) New. Limbs, flipper-like, indicated by the presence of rod-like stylopodial and zygapodial 3009 elements, simple disc-like tarsal and carpal bones, and hyperphalangy: absent (0); present (1).

3010 In Dinocephalosaurus orientalis (Rieppel et al. 2008) the limbs have been modified to function as flippers 3011 for aquatic propulsion. The presence of at least one more Dinocephalosaurus-like taxon (Li et al. 2017b) 3012 indicates that more non-archosauriform archosauromorphs might have had flippers.

3013 240) New. Long bone histology, fibrolamellar bone tissue in the cortex: absent (0); present (1).

3014 Early archosauromorphs exhibit considerable variation in their bone tissue. Fibrolamellar tissue is 3015 present in the cortex of Azendohsaurus (Cubo \& Jalil 2019), Prolacerta, Proterosuchus, and 3016 Erythrosuchus (Botha-Brink \& Smith) among the sampled genera, but absent in the non-saurian diapsid 3017 Claudiosaurus (De Buffrénil \& Mazin 1989) and the archosauromorphs Macrocnemus and Tanystropheus 3018 (Jaquier \& Scheyer 2017), Trilophosaurus buettneri (Werning \& Irmis 2010), and Euparkeria (Botha-Brink 3019 \& Smith 2011). The presence of fibrolamellar bone tissue can contain a strong phylogenetic signal, as it 3020 has important implications for growth rates and metabolism. Therefore, this character has been 3021 included in a phylogenetic context here for the first time.

3022 241) Ezcurra (2016) ch. 415 . Humerus, torsion between proximal and distal ends: approximately 45 3023 degrees or more (0); 35 degrees or less (1) (Ezcurra 2016: Fig. 39).

3024 242) Ezcurra (2016) ch. 416. Humerus, transverse width of the proximal end versus total length of the 3025 bone in skeletally mature individuals: 0.11-0.33 (0); 0.38-0.46 (1); 0.56-0.68 (2), ORDERED RATIO (Ezcurra 3026 2016: Fig. 39).243) Ezcurra (2016) ch. 420. Humerus, conical process on the proximal surface, placed 
3027 immediately adjacent to the base of the deltopectoral crest: absent (0); present (1) (Ezcurra 2016: Fig. 3028 39).

3029 244) Ezcurra (2016) ch. 423. Humerus, ventral margin of the deltopectoral crest developed as a thick 3030 subcilindrical tuberosity that is well-differentiated from the thinner dorsal margin: present (0); absent (1) 3031 (Ezcurra 2016: Fig. 39).

3032 245) Ezcurra (2016) ch. 425. Humerus, entepicondyle size in skeletally mature individuals: moderately 3033 large (0); strongly developed (1) (Ezcurra 2016: Fig. 39).

3034 246) Ezcurra (2016) ch. 426 and Pritchard et al. (2015) ch. 153. Humerus, entepicondylar foramen: 3035 present (0); absent (1) (Ezcurra 2016: Fig. 39).

3036 247) Ezcurra (2016) ch. 427. Humerus, ectepicondylar region: foramen present (0); foramen absent, 3037 supinator process and groove present (1); supinator process, groove or foramen absent (2) (Ezcurra 3038 2016: Fig. 39).

3039 248) Modified from Ezcurra (2016) ch. $414 . \quad$ Humerus, total length of the humerus versus the total 3040 length of the femur: 0.63-0.71 (0); 0.76-0.80 (1); 0.84-0.91 (2); 0.97-1.05 (3), ORDERED RATIO.

3041 This character is modified to compare the total length of the humerus to the femur rather than the 3042 entire forelimb to the entire hindlimb.

3043 249) Pritchard et al. (2015) ch. 157 and Ezcurra (2016) ch. 430 (reformulated). Ulna, olecranon process: 3044 absent, not ossified or very low in skeletally mature individuals (0); present (1) (Ezcurra 2016: Fig. 40).

3045 250) Ezcurra (2016) ch. 433. Ulna, lateral tuber (=radius tuber) on the proximal portion: absent in 3046 skeletally mature individuals (0); present (1) (Nesbitt 2011: Figs. 40 and 31).

3047 251) Ezcurra (2016) ch. 435. Radius, total length versus total length of the humerus: 0.53-0.72 (0); 3048 0.81-0.92 (1); 1.01-1.07 (2); 1.40-1.46 (3), ORDERED RATIO (Ezcurra 2016: Fig. 15).252) Ezcurra (2016) 3049 ch. 440 and Pritchard et al. (2015) ch. 161. Carpals, perforating foramen between intermedium and 3050 ulnare: present (0); absent in skeletally mature individuals (1). This character is inapplicable in taxa that 3051 lack an intermedium.

3052 253) New, combination of ch. 441 and 442 of Ezcurra (2016). Centrale of the manus of skeletally 3053 mature individuals: both the lateral and medial centrale are present (0); only the lateral centrale is 3054 present (1); only the medial centrale is present (2); both are absent (3). 
3055

3056

3057

3058

3059

3060

3061

3062

3063

3064

3065

3066

3067

3068

3069

3070

3071

3072

3073

3074

3075

3076

3077

3078

3079

3080

3081

3082

254) Ezcurra (2016) ch. 443. Carpals, pisiform: present (0); absent in skeletally mature individuals (1) (Ezcurra 2016: Fig. 40).

255) Benton \& Allen (1997) ch. 31 and Jalil (1997) ch. 46.

First distal carpal: present (0); absent in skeletally mature individuals (1).

256) Ezcurra (2016) ch. 444 and Pritchard et al. (2015) ch 159. Carpals, distal carpal five: absent in skeletally mature individuals (0); present (1) (Ezcurra 2016: Fig. 40).

257) Ezcurra (2016) ch 445. $\quad$ Manus, longest metacarpal + digit: longer than humeral length (0); subequal to or shorter than humeral length (1).

258) Ezcurra (2016) ch. 446. Metacarpus, length of the longest metacarpal versus length of the longest metatarsal: 0.32-0.33 (0); 0.36-0.41 (1); 0.46-0.49 (2); 0.61-0.65 (3), ORDERED RATIO.

259) Ezcurra (2016) ch. 448. Metacarpus, width of the distal end of the metacarpal I versus its total length: 0.25-0.33 (0); 0.38-0.46 (1); 0.49-0.50 (2); 0.56-0.61 (3); 0.65-0.67 (4), ORDERED RATIO (Ezcurra 2016: Fig. 40).260) Ezcurra (2016) ch. 450 . Metacarpus, metacarpal IV: longer than metacarpal III (0); equal to or shorter than metacarpal III (1) (Ezcurra 2016: Fig. 40).

261) Ezcurra (2016) ch. 453. Manual digits, second phalanx of manual digit II: shorter than the first phalanx of manual digit II (0); longer than the first phalanx of manual digit II (1) (Nesbitt 2011: Fig. 32).

262) Ezcurra (2016) ch. 451 and Nesbitt et al. (2015) ch. 222. Manual digits, unguals length: about the same length or shorter than the last non-ungual phalanx of the same digit (0); distinctly longer than the last non-ungual phalanx of the same digit (1). This character is inapplicable in taxa in which the terminal phalanx of each digit does not form an ungual.

An inapplicability criterion was added because in the aquatic Dinocephalosaurus orientalis no ungual is formed by the terminal phalanges.

263) Modified from Ezcurra (2016) ch. 454. Manual digits, number of phalanges in digit IV: five (0); four (1) (Nesbitt 2011: Fig. 32).

State 2 of character 454 in Ezcurra (2016) was removed because it was irrelevant for the sampled taxa.

264) Modified from Ezcurra (2016) ch $460 . \quad$ Ilium, preacetabular process: absent or incipient (0); present, being considerably anteroposteriorly shorter than its dorsoventral height (1); present, being longer than two thirds of its height (2), ORDERED. 
3083 States 2 and 3 of character 460 in Ezcurra (2016) were fused and redescribed to address the specific 3084 morphology observed in the sampled taxa.

3085 265) Modified from Sookias (2016) ch. 268. Ilium, shape of preacetabular process: rounded (0); 3086 approximately straight-sided with a distinct angle between the anterior and dorsal margins (1). This 3087 character is inapplicable in taxa that lack a preacetabular process on the ilium.

3088 States 1 and 2 were fused and state 3 was removed compared to the original character of Sookias (2016) 3089 to specifically address the observed morphologies in the sampled taxa.

3090 266) Modified from Pritchard et al. (2015) ch. 170 and Ezcurra (2016) ch. 461. Ilium, anterior 3091 process/tuber on the anterior margin of the ilium: anterior process/tuber absent or incipient (0); clearly 3092 defined anteriorly projecting tuber on the anterior margin of the preacetabular process (1) (Fig. 32). This 3093 character is inapplicable in taxa that lack a preacetabular process on the ilium.

3094 Pritchard et al. (2015) considered the presence of a small tuber on the anterior margin of the iliac blade 3095 in certain tanystropheids to represent the same structure as the preacetabular process, which was 3096 incorporated into character 170 of their dataset. This character interpreted the presence of an 3097 anteriorly expanded preacetabular process to represent a more strongly exhibited version of this tuber. 3098 However, we consider this tuber to represent a separate structure on the preacetabular process, since 3099 this tuber occurs in certain taxa that also have a smooth, anterodorsally curved preacetabular process in 3100 lateral view. The presence of a small finger-like tuber on the anterior margin of the preacetabular 3101 process is subject to intraspecific variation and occurs in Tanystropheus longobardicus (PIMUZ T 1277), a 3102 juvenile specimen of Macrocnemus bassanii (MSNM BES SC 111), and Fuyuansaurus acutirostris (IVPP 3103 V17983) among the sampled taxa and it is considered to represent an informative character 3104 independent of the size of the preacetabular process. This tuber is also present in the nomen dubium 3105 "Exilisuchus tubercularis" (Ochev 1979; Fig. 9 of Ezcurra 2016). "Exilisuchus tubercularis" is exclusively 3106 known from a partial ilium and was recovered as a tanystropheid in the phylogenetic analysis of Ezcurra 3107 (2016).

3108 267) Ezcurra (2016) ch. 463 (reformulated). Ilium, length of the postacetabular process measured 3109 from the most proximal point on the posterior/ventral margin of the process versus anteroposterior 3110 length of the acetabulum: 0.50-0.71 (0); 0.88-0.91 (1); 0.98-1.14 (2); 1.22-1.57 (3); $1.72-1.78$ (4), 3111 ORDERED RATIO (Ezcurra 2016: Fig. 41). 
3112 The character was reformulated to specify the measurement of the length of the postacetabular 3113 process.

3114 268) Ezcurra (2016) ch. 464 and Pritchard et al. (2015) ch. 164. Ilium, main axis of the postacetabular 3115 process in lateral or medial view: posterodorsally orientated (0); mainly posteriorly orientated (1) 3116 (Ezcurra 2016: Figs. 9 and 41).

3117 269) Modified from Ezcurra (2016) ch. 465 . Ilium, caudifemoralis brevis muscle origin on the 3118 lateroventral surface of the postacetabular process: not dorsally or laterally rimed by a brevis shelf $(0)$; 3119 dorsally rimed by a low brevis shelf (1) (Ezcurra 2016: Fig. 9).

3120 State 0 of character 465 in Ezcurra (2016) has been removed because it is irrelevant to the sampled taxa. 3121 270) Pritchard et al. (2015) ch. 166. Ilium, supra-acetabular crest: crest absent, anterodorsal margin 3122 of acetabulum similar in development to posterodorsal margin (0); prominent anterodorsal lamina 3123 frames the anterodorsal margin of the acetabulum (1).

3124 271) Pritchard et al. (2015) ch. $167 . \quad$ Ilium, shape of supra-acetabular margin: dorsalmost margin of 3125 acetabulum is unsculptured (0); prominent, bulbous rugosity superior to acetabulum (1). This character is 3126 inapplicable in taxa that lack a distinct supra-acetabular crest.

3127 272) Pritchard et al. (2015) ch. 165 . Ilium, anteroventral process extending from anterior margin of 3128 pubic peduncle: absent (0); present, process draping across anterior surface of pubis (1).

3129 273) Ezcurra (2016) ch. 471 and Pritchard et al. (2015) ch. 163. Pubis-ischium, thyroid fenestra: absent 3130 (0); present (1) (Ezcurra 2016: Fig. 41).

3131 274) Pritchard et al. (2015) ch. $175 . \quad$ Pubis, lateral surface, development of a lateral tubercle (sensu 3132 Vaughn 1955): present (0); absent (1).

3133 275) Ezcurra (2016) ch. $477 . \quad$ Pubis, pubic apron: absent, symphysis extended along the ventral margin 3134 of the pelvic girdle and visible in lateral view (0); present, symphysis restricted anteriorly and obscured by 3135 the pubic shaft in lateral view (1) (Ezcurra 2016: Fig. 41).

3136 This character is discussed on page 61 and figured in Fig. 58 of Nesbitt et al. (2015).

3137 276) Ezcurra (2016) ch. 482 and Nesbitt et al. (2015) ch. 225 . Ischium, maximal length versus 3138 anteroposterior length of the acetabulum: 1.46-1.57 (0); 1.67-1.77 (1); 1.86-1.98 (2); 2.06-2.23 (3); 2.51- 
31392.89 (4), ORDERED RATIO.277) Ezcurra (2016) ch. 486. Ischium, symphysis raised on a distinct low 3140 peduncle: absent (0); present (1) (Ezcurra 2016: Fig. 41).

3141 This character is discussed on page 62 of Nesbitt et al. (2015).

3142 278) Modified from Pritchard et al. (2015) ch. 176 and Ezcurra (2016) ch. 488. Ischium, distinct

3143 concavity or constriction on the posterior half of the ventral margin of the ischium, thus separating a 3144 distinct posterior process from the rest of the ischium: absent (0); present (1) (Fig. 32; Ezcurra 2016: Fig. 3145 41).

3146 This character was discussed by Pritchard et al. (2015), where it was considered homologous to the 3147 spina ischia described by El-Toubi (1949). We reformulate the character based on our observations of 3148 the ischia in the sampled taxa. A posterior process of the ischium is formed by a distinct concavity or 3149 constriction of the ventral margin of the ischium in Planocephalosaurus robinsonae, Pectodens 3150 zhenyuensis, and Langobardisaurus pandolfii. Furthermore, this trait is also present in some, but not all, 3151 specimens of Macrocnemus bassani, Macrocnemus fuyuanensis, Tanystropheus longobardicus, and 3152 Amotosaurus rotfeldensis. Therefore, this character clearly shows a large amount of intraspecific 3153 variability.

3154 279) Ezcurra (2016) ch. 491 . Femur, proximal articular surface in skeletally mature individuals: well3155 ossified, being flat or convex (0); partially ossified, being concave and sometimes with a circular pit (1) 3156 (Ezcurra 2016: Fig. 42).

3157 280) Ezcurra (2016) ch. 504. Femur, attachment of the caudifemoralis musculature on the posterior 3158 surface of the bone: crest-like and with intertrochanteric fossa (=internal trochanter), and convergent 3159 with proximal end (0); crest-like and with intertrochanteric fossa (=internal trochanter), and not 3160 convergent with proximal end (1); crest-like and without intertrochanteric fossa (=fourth trochanter), 3161 and not convergent with proximal end (2) (Ezcurra 2016: Figs. 42 and 43). This character is inapplicable 3162 in taxa without a distinct process for the attachment of the caudifemoralis musculature on the femur.

3163 Character 504 in Ezcurra (2016) is ordered. We decided not to order this character here because we do 3164 not consider it clear that the states represent intermediate steps in a transformational series.

3165 281) Ezcurra (2016) ch. 511 . Femur, distal condyles: prominent, strong dorsoventral expansion (in 3166 sprawling orientation) restricted to the distal end (0); not projecting markedly beyond shaft and expand 3167 gradually if there is any expansion (1) (Ezcurra 2016: Fig. 43). 
3168 See description of character 318 in Nesbitt (2011).

3169 282) Ezcurra (2016) ch. 512. Femur, distal articular surface: uneven, lateral (=fibular) condyle

3170 projecting distally distinctly beyond medial (=tibial) condyle (0); both condyles prominent distally and

3171 approximately at same level (1); both condyles do not project distally (distal articular surface concave or 3172 almost flat) (2) (Ezcurra 2016: Figs. 42 and 43).

3173 283) Ezcurra (2016) ch. 513. Femur, anterior extensor groove: absent, anterior margin of the bone 3174 straight or convex in distal view (0); present, anterior margin of the bone concave in distal view (1) 3175 (Ezcurra 2016: Fig. 42).

3176 284) Ezcurra (2016) ch. 515. Femur, shape of lateral (=fibular) condyle in distal view: lateral surface is 3177 rounded and mound-like (0); lateral surface is triangular and sharply pointed (1) (Ezcurra 2016: Fig. 42).

3178 285) Benton \& Allen (1997) ch. 39. Femur, length of tibia relative to length of femur: tibia shorter 3179 than, or subequal to, femur in length (0); tibia longer than femur (1).

3180 286) Pritchard et al. (2015) ch. 177. Femur, shape in lateral view: femoral shaft exhibits sigmoidal 3181 curvature (0); femoral shaft linear with slight ventrodistal curvature (1).

3182 287) Ezcurra (2016) ch. 528. Fibula, transverse width at mid-length: subequal to transverse width of 3183 the tibia (0); distinctly narrower than transverse width of the tibia (1) (Ezcurra 2016: Fig. 15).

3184 288) Ezcurra (2016) ch. 531. Fibula, distal end in lateral view: angled anterodorsally (asymmetrical) 3185 (0); rounded or flat (symmetrical) (1) (Ezcurra 2016: Fig. 44, Nesbitt 2011: Fig. 41).

3186 289) Modified from Ezcurra (2016) ch. 532. Proximal tarsals, articulation between astragalus and 3187 calcaneum: roughly flat (0); concavoconvex with concavity on the astragalus (1); fused (2) (Ezcurra 2016: 3188 Fig. 45).

3189 State 1 of character 532 of Ezcurra (2016) is excluded because it does not apply to the sampled taxa.

3190 This character can best be observed in plantar view. For a detailed description of the articulation 3191 between the astragalus and calcaneum, see the extensive discussion in Sereno (1991), in particular Figs. 3192 3-4, 8 therein, and Cruickshank (1979).

3193 290) Ezcurra (2016) ch. 539. Astragalus, posterior groove: present (0); absent (1) (Nesbitt 2011: Fig. 3194 46). 
3195 This character is extensively discussed in page. 353 of Gower (1996). Due to the three-dimensional

3196 structure of the astragalus and the variation observed in its morphology in the sampled taxa, it is hard to 3197 distinguish the groove from other curves and concavities in the bone in certain taxa, since this character 3198 was originally formed to describe the astragalus of archosauriforms. Taxa are scored as 0 when a clear 3199 concavity is present on the ventral/plantar surface of the astragalus, often connecting to the perforating 3200 foramen between the astragalus and calcaneum.

3201 291) Modified from Pritchard et al. (2015) ch. 184 and part of Ezcurra (2016) ch. 557. Distal tarsals, 3202 pedal centrale: present or partially fused to the astragalus in mature individuals (0); absent as a separate 3203 ossification, being either unossified or fused to the astragulus in skeletally mature individuals (1) (Ezcurra 3204 2016: Figs. 45 and 46).

3205 In character 184 of Pritchard et al. (2015) the absence of a pedal centrale is stated to result from the 3206 fusion of this element to the astragalus. It has indeed been documented that the loss of a separate 3207 pedal centrale in several early archosauromorphs is the result of fusion with the astragalus (Fernández 3208 Blanco et al. 2020). However, in Tanystropheus spp. and in Dinocephalosaurus orientalis the absence of 3209 the pedal centrale is more likely to be attributable to skeletal paedomorphosis related to aquatic 3210 adaptations (Rieppel 1989; Rieppel et al. 2008). A pedal centrale is also absent in the pedes of the only 3211 known specimen of Pectodens zhenyuensis (Li et al. 2017a). However, since this specimen might 3212 represent an early ontogenetic stage, we refrain from scoring this character for this taxon. In the 3213 absence of sufficient embryological data to assess its developmental underpinnings for our taxonomic 3214 sample, our character addresses the presence or absence of this element irrespective of whether it 3215 might be attributable to paedomorphosis or fusion with the astragalus.

3216 292) New, combination of Ezcurra (2016) ch. 558, same as Pritchard et al. (2015) ch. 193, and Ezcurra 3217 (2016) ch. 559, same as Pritchard et al. (2015) ch. 194. Distal tarsals of skeletally mature individuals, 3218 distal tarsal 1 and 2: both present (0); only one of the two elements is present (1); both absent (2), 3219 ORDERED.

3220 Characters 558 and 559 in Ezcurra (2016) were fused here, because in certain taxa (Macrocnemus 3221 bassanii, PIMUZ T 4822; Macrocnemus fuyuanensis, IVPP V15001; and Amotosaurus rotfeldensis, SMNS $322254783 \mathrm{a} / \mathrm{b}$ ) one of the distal tarsals is present, but it cannot be established confidently whether this 3223 represents distal tarsal 1 or 2 . We consider this of secondary importance, as this character treats with 3224 the degree of ossification (paedomorphosis) in the tarsus, and both distal tarsals ossify at roughly the 
3225 same developmental stage (Rieppel 1989). This character has been ordered because state 2, the

3226 absence of both elements, represents a larger degree of paedomorphosis than state 1, the absence of

3227 only a single element. Therefore, state 1 is considered to represent an intermediate step between states

32280 and 2.

3229 293) Ezcurra (2016) ch. 563 and Pritchard et al. (2015) ch. 195. Distal tarsals, distal tarsal 5: present (0);

3230 absent in skeletally mature individuals (1).

3231

294) Modified from Ezcurra (2016) ch. 564. Pes, foot length (articulated fourth metatarsal and digit) versus tibia-fibula length: 0.60-0.68 (0); 0.79-1.04 (1); 1.12-1.16 (2); 1.34-1.56 (3); 1.96-2.04 (4), ORDERED RATIO (Ezcurra 2016: Fig. 15).

3234

The original distinction between the character states was not considered to be phylogenetically relevant for the sampled taxa and therefore it was decided to distinguish states based on calculated ratios.

295) Ezcurra (2016) ch. 533 and Pritchard et al. (2015) ch. 186. Proximal tarsals, foramen for the passage of the perforating artery between the astragalus and calcaneum (=perforating foramen): present (0); absent in skeletally mature individuals (1) (Ezcurra 2016: Fig. 45).

296) Ezcurra (2016) ch. 565. Metatarsus, configuration: metatarsals diverging from ankle (0); compact, metatarsals I-IV tightly bunched (1) (Ezcurra 2016: Fig. 46).

297) Ezcurra (2016) ch. 569. Metatarsus, length of metatarsal I versus metatarsal III: 0.36-0.43 (0); 0.48-0.51 (1); 0.54-0.63 (2); 0.67-0.75 (3); 0.82-0.84 (4), ORDERED RATIO (Ezcurra 2016: Fig. 46).

298) Ezcurra (2016) ch. 571. Metatarsus, length of the metatarsal II versus length of the metatarsal IV: 0.55-0.67 (0); 0.70-0.76 (1); 0.80-0.86 (2); 0.89-0.91 (3); 0.94-1.02 (4), ORDERED RATIO (Ezcurra 2016: Fig. 46).299) Ezcurra (2016) ch. 574. Metatarsus, length of metatarsal IV versus length of metatarsal III: 0.88-1.00 (0); 1.03-1.08 (1); 1.13-1.22 (2); 1.25-1.26 (3), ORDERED RATIO (Ezcurra 2016: Fig. 46). Character 581 in Ezcurra (2016) is not included in our analysis, because the length of the entire digit is strongly dependent on the length of the metatarsal, and these characters are therefore considered interdependent. It was preferred to compare the relative lengths of the metatarsals over the lengths of the entire digits because this feature could be scored in more of the sampled taxa.

300) Ezcurra (2016) ch. 577 (reformulated). Metatarsus, metatarsal V with a hook-shaped proximal end: absent (0); present, with a gradually medially curved proximal process (1); present, with an abruptly 
3253 medially flexed proximal process and, as a result, the metatarsal acquires a L-shape in dorsal or ventral 3254 view (2) (Ezcurra 2016: Fig. 46).

3255 301) Ezcurra (2016) ch. 576 (reformulated). Metatarsus, dorsal prominence separated from the 3256 proximo-medial surface by a concave gap in metatarsal V: absent (0); present (1) (Ezcurra 2016: Fig. 46, 3257 Nesbitt 2011: Fig. 47). This character is inapplicable in taxa that lack a hook-shaped metatarsal V.

3258 302) Ezcurra (2016) ch. 578 and Pritchard et al. (2015) ch. 196. Metatarsus, metatarsal V outer process 3259 on the proximal lateral margin: absent, smooth curved margin (0); present, prominent pointed process 3260 (1)

3261 303) Ezcurra (2016) ch. 579. Metatarsus, metatarsal V lateral plantar tubercle: absent (0); present (1) 3262 (Ezcurra 2016: Fig. 46).

3263 304) Ezcurra (2016) ch. 580. Metatarsus, metatarsal V medial plantar tubercle: absent (0); present (1) 3264 (Ezcurra 2016: Fig. 46).

3265 305) Modified from Benton \& Allen (1997) ch. $45 . \quad$ Metatarsus, length of metatarsal IV versus the 3266 proximodistal length of metatarsal V: 1.25-1.90 (0); 2.19-2.57 (1); 2.83-3.25 (2); 3.65-5.15 (3), ORDERED 3267 RATIO.The original distinction between the character states was not considered to be phylogenetically 3268 relevant for the sampled taxa and therefore it was decided to distinguish states based on calculated 3269 ratios.

3270 306) Ezcurra (2016) ch. 584 and Pritchard et al. (2015) ch. 199. Pedal digits, phalanx V-1: subequal to or 3271 shorter than other non-ungual phalanges (0); metatarsal-like, considerably longer than other non-ungual 3272 phalanges (1).

3273 307) Ezcurra (2016) ch 587 and Nesbitt et al. (2015) ch. 233. Pedal digits, ventral tubercle in unguals: 3274 absent or small (0); well-developed and extended ventral to the articular portion of the ungual (1).

\section{Results}

3276

3277

3278

3279

3280

Analyses excluding ratio characters and all remaining characters treated as unordered

Analyses 1 and 2 both exclude ratio characters and treat all characters as unordered. Analysis 1 , which includes all OTUs, found 1976 MPTs of 977 steps with a consistency index (CI) of 0.370 and a retention index (RI) of 0.534, whereas analysis 2, which excluded the problematic OTUs Czatkowiella harae, Tanystropheus "conspicuus", and "Tanystropheus antiquus", recovered 884 MPTs that had 953 
3281 steps and a Cl of 0.379 and a RI of 0.537. Support values for the branches are indicated in the SCTs (Figs. $328233 a$ and 34a). The SCTs of both analyses contain large polytomies, which are consecutively more resolved 3283 in the three sequential RSCTs calculated for each analysis (Figs. 33b-d and 34b-d). At the base of the SCTs of both analyses a polytomy is formed by Claudiosaurus germaini, Acerosodontosaurus piveteaui, Youngina capensis, and Sauria, with Orovenator mayorum as the sister taxon to this polytomy (Figs. 33a and 34a). Although it is generally considered that Orovenator mayorum is most distantly related to Sauria among our sampled OTUs except for the outgroup Petrolacosaurus kansensis (Ford \& Benson 2020; Reisz et al. 2011), there is no clear consensus in the relationships between Youngina capensis, Claudiosaurus germaini, and Acerosodontosaurus piveteaui (Bickelmann et al. 2009). Since the focus of this study is not to resolve early diapsid phylogeny, only these taxa, which are among the morphologically best-known early diapsids, were included, and the lack of resolution can possibly be attributed to the low taxonomic and character samples for this part of the tree. All MPTs of both analyses recover a monophyletic Sauria, Lepidosauromorpha, Archosauromorpha, Crocopoda, Rhynchosauria, Allokotosauria, and

Archosauriformes.

In the SCTs of both analyses a huge polytomy is formed at the base of Archosauromorpha that includes the monophyletic Crocopoda and all remaining "protorosaurs", some of which are recovered in less inclusive clades within the polytomy (Figs. 33a and 34a). The SCT of analysis 1 recovers Macrocnemus bassanii and Macrocnemus fuyuanensis as sister taxa but finds the poorly known Macrocnemus obristi outside this clade. The SCT of analysis 2 does not recover Macrocnemus bassanii and Macrocnemus fuyuanensis in a generic clade. In the SCT of analysis 1 a polytomic clade is formed by all Tanystropheus spp. except "Tanystropheus antiquus" and additionally including Raibliania calligarisi. The SCT of analysis 2, from which both Tanystropheus "conspicuus" and "Tanystropheus antiquus" were omitted a priori, also recovers a polytomic clade of all remaining Tanystropheus OTUs and Raibliania calligarisi. Another clade formed by OTUs generally considered to be tanystropheid is recovered in the SCTs of both analyses. In analysis 1 this clade is formed by Langobardisaurus pandolfii and Tanytrachelos ahynis and in analysis 2 it is formed by AMNH FARB 7206, Langobardisaurus pandolfii, and Tanytrachelos ahynis as successive sister taxa. The remaining "protorosaurs" do not form more inclusive clades within the large polytomy at the base of Archosauromorpha in both SCTs. sister groups being formed by Prolacerta broomi, Allokotosauria, Rhynchosauria, Teyujagua paradoxa, and Archosauriformes in all MPTs (Fig. 33a). In the SCT of analysis 2 Prolacerta broomi is the sister taxon 
3312 to a polytomy formed by Teyujagua paradoxa, Allokotosauria, Rhynchosauria, and Archosauriformes (Fig. 3313 34a). The position of Prolacerta broomi as the crocopodan most distantly related to Archosauriformes 3314 differs from other recent analyses in which Prolacerta broomi was found to be more closely related to 3315 Archosauriformes than both rhynchosaurs and allokotosaurs (e.g., Ezcurra 2016; Nesbitt et al. 2015; 3316 Pinheiro et al. 2019; Pritchard et al. 2018; Pritchard \& Sues 2019; Pritchard et al. 2015; Spiekman 2018). 3317 The relationships within Allokotosauria, Rhynchosauria, and Archosauriformes are the same between 3318 both analyses (Figs. 33a and 34a) and are in congruence with that of previous phylogenetic studies. Within 3319 Allokotosauria Pamelaria dolichotrachela is the sister taxon to the clade formed by Trilophosaurus 3320 buettneri and Azendohsaurus madagaskarensis. Rhynchosauria consists of Mesosuchus browni, 3321 Eohyosaurus wolvaardti, and Howesia browni as successive sister taxa. Within Archosauriformes 3322 Euparkeria capensis is the sister taxon to Erythrosuchus africanus and a generic clade is formed by Proterosuchus fergusi and Proterosuchus alexanderi.

The iter PCR option found four unstable OTUs for analysis 1 . The first RSCT of analysis 1 was generated after the exclusion a posteriori of Acerosodontosaurus piveteaui and Macrocnemus obristi (Fig. 33b). In this RSCT the relationships of non-saurian diapsids are resolved, with Claudiosaurus germaini forming the sister taxon to Youngina capensis + Sauria. The a posteriori pruning of Macrocnemus obristi increases the resolution among non-archosauriform archosauromorphs. Protorosaurus speneri and Jesairosaurus lehmani form successive sister taxa to all other archosauromorphs. An additional clade is also recovered among non-archosauriform archosauromorphs, formed by a polytomy of "Tanystropheus antiquus", Sclerostropheus fossai, Pectodens zhenyuensis, and Dinocephalosaurus orientalis. This clade is the sister group to the remainder of the large polytomy that is present in RSCT 1. "Tanystropheus antiquus" and Sclerostropheus fossai have generally been recognized as tanystropheids closely related to Tanystropheus spp. (Spiekman \& Scheyer 2019). The phylogenetic placement of the Chinese "protorosaurs" Dinocephalosaurus orientalis and Pectodens zhenyuensis has been more elusive (Li et al. 2017a; Rieppel et al. 2008), although they have been tentatively referred to Tanystropheidae in some studies (Ezcurra \& Butler 2018; Li 2003; Liu et al. 2017).

The RSCT 2 of analysis 1 was generated after the additional a posteriori pruning of Elessaurus gondwanoccidens, which further increases the resolution among non-archosauriform archosauromorphs 3340 (Fig. 33c). In this RSCT a monophyletic Tanystropheidae clade is recovered. A generic clade of 3341 Macrocnemus bassanii and Macrocnemus fuyuanensis forms the sister group to all other taxa forming 
3343 sister groups and together form the sister clade to all remaining members of the Tanystropheidae clade 3344 except the included Macrocnemus OTUs. Augustaburiania vatagini and Amotosaurus rotfeldensis form 3345 successive sister groups to the polytomic clade composed of Raibliania calligarisi and the Tanystropheus 3346 OTUs except "Tanystropheus antiquus". An additional clade is formed by Czatkowiella harae and Ozimek 3347 volans. This clade is found as the sister group to a clade composed of Fuyuansaurus acutirostris, 3348 Tanystropheidae, and Crocopoda.

The a posteriori pruning of Pectodens zhenyuensis additionally found "Tanystropheus antiquus" and Sclerostropheus fossai as sister taxa, with Dinocephalosaurus orientalis forming the sister taxon to this clade in RSCT 3 of analysis 1 (Fig. 33d). The exclusion of Pectodens zhenyuensis is remarkable, since this OTU possesses a low amount of missing data (45.6\%) compared to other OTUs in that clade (e.g., Sclerostropheus fossai 4.9\%, and "Tanystropheus antiquus" 5.2\%; Table 1).

For analysis 2 nine OTUs were excluded by the iter PCR function. As for analysis 1, RSCT 1 of analysis 2 was calculated after the a posteriori exclusion of Acerosodontosaurus piveteaui and Macrocnemus obristi and the relationships among non-saurian diapsids match those of RSCT 1 of analysis 1 (Fig. 34b). The exclusion of Macrocnemus obristi results in the formation of a clade that includes all remaining "protorosaurs" except Protorosaurus speneri, Jesairosaurus lehmani, and Prolacerta broomi. The relationships within this new clade are not further resolved relative to the SCT. This large and poorly resolved clade forms a polytomy with Protorosaurus speneri, Jesairosaurus lehmani, and Crocopoda. acutirostris, and Dinocephalosaurus orientalis are additionally excluded a posteriori in RSCT 2 and a monophyletic Tanystropheidae is recovered as a consequence. As for RSCT 3 of analysis 1, despite possessing a relatively low amount of missing data Pectodens zhenyuensis is pruned (Table 1), in addition to Dinocephalosaurus orientalis, which posesses even fewer missing data (64.8\%). The monophyletic Tanystropheidae recovered in RSCT 2 of analysis 2 is virtually identical to that of RSCTs 2 and 3 of analysis 1. However, in contrast to these analyses, the Tanystropheidae clade of RSCT 2 of analysis 2 includes Ozimek volans, which forms a trichotomy with Amotosaurus rotfeldensis and a clade containing all included Tanystropheus OTUs and Raibliania calligarisi (Fig. 34c). This last clade differs from that of RSCTs analysis. 
In RSCT 3 of analysis 2 two additional OTUs were pruned a posteriori, Jesairosaurus lehmani and

3373

3374

3375

3376

3377

3378

3379

3380

3381

3382

3383

3384

3385

3386

3387

3388

3389

3390

3391

3392

3393

3394

3395

3396

3397

3398

3399

3400

3401

3402

GMPKU P 1527. The exclusion of the former results in a topology of Protorosaurus speneri, Tanystropheidae, and Crocopoda as successive sister groups among non-archosauriform archosauromorphs (Fig. 34d). The exclusion of GMPKU P 1527, currently assigned to Tanystropheus cf. $T$. hydroides, resolves the relationships between Tanystropheus hydroides, Tanystropheus longobardicus, and Raibliania calligarisi, with the last two being sister taxa.

Analyses including ratio characters and treating designated characters as ordered

Analysis 3, which includes all OTUs and incorporates ratio and ordered characters (Fig. 35), found 434 MPTs of 1270 steps and has a $\mathrm{Cl}$ of 0.350 and a RI of 0.516 , whereas analysis 4, which incorporates ratio and ordered characters and excludes Czatkowiella harae, Tanystropheus "conspicuus", and "Tanystropheus antiquus" (Fig. 36), recovered 154 MPTs of 1241 steps and a Cl of 0.358 and a RI of 0.518. Support values for the branches are indicated in the SCTs of both analyses (Figs. 35a and 36a). The SCTs of both analyses show a higher resolution compared to those of analyses 1 and 2 and recover a monophyletic Tanystropheidae, as well as a new clade of non-archosauriform archosauromorphs composed of at least Pectodens zhenyuensis and Dinocephalosaurus orientalis. However, both SCTs contain a large polytomy within Tanystropheidae. Three consecutively more resolved RSCTs were calculated for analysis 3 (Fig. 35b-d), whereas only a single RSCT could be found for analysis 4 (Fig. 36b). The relationships among non-saurian diapsids are fully resolved in the SCT of analysis 3, with Petrolacosaurus kansensis, Orovenator mayorum, Claudiosaurus germaini, Acerosodontosaurus piveteaui, Youngina capensis, and Sauria forming successive sister groups (Fig. 35a). Even though there are no differences in character configuration with analysis 3, in this part of the SCT of analysis 4 a polytomy is formed by all these taxa except for the outgroup Petrolacosaurus kansensis (Fig. 36a). As in analyses 1 and 2, a monophyletic Sauria, Lepidosauromorpha, Archosauromorpha, Crocopoda, Rhynchosauria, Allokotosauria, and Archosauriformes is recovered in all MPTs of analyses 3 and 4 (Figs. 35a and 36a). However, in contrast to the previous analyses, the SCTs of analyses 3 and 4 both did not recover Prolacerta broomi within Crocopoda. This contrasts distinctly with recent phylogenetic analyses in which Prolacerta broomi has been uniformally found to be very closely related to Archosauriformes (e.g., Ezcurra 2016; Nesbitt et al. 2015; Pinheiro et al. 2019; Pritchard et al. 2018; Pritchard \& Sues 2019; Pritchard et al. 2015; Spiekman 2018). Furthermore, whereas the SCTs analyses 1 and 2 found large polytomies at the base of non-archosauriform archosauromorphs, those of analyses 3 and 4 show a higher resolution and in all MPTs of both analyses a monophyletic Tanystropheidae is recovered, as well as a previously unrecognized 
3403 clade. In the SCT of analysis 3 this clade is formed by a polytomy of Pectodens zhenyuensis, 3404 Dinocephalosaurus orientalis, and "Tanystropheus antiquus" (Fig. 35a). In the SCT of analysis 4 it is formed

3405

3406

3407

3408

3409

3410

3411

3412

3413

3414

3415

3416

3417

3418

3419

3420

3421

3422

3423

3424

3425

3426

3427

3428

3429

3430

3431

3432

3433 only by Pectodens zhenyuensis and Dinocephalosaurus orientalis, with Jesairosaurus lehmani forming the sister taxon to this clade (Fig. 36a). In all MPTs of analysis 3 Jesairosaurus lehmani, Protorosaurus speneri, and Prolacerta broomi form successive sister groups leading up to a clade composed of all remaining nonarchosauriform archosauromorphs (Fig. 35a). This last clade is split into all taxa previously considered as crocopodans except Prolacerta broomi, and a clade encompassing Tanystropheidae, Czatkowiella harae, Ozimek volans, Fuyuansaurus acutirostris, and the new clade formed by Pectodens zhenyuensis, Dinocephalosaurus orientalis, and "Tanystropheus antiquus". Fuyuansaurus acutirostris is the sister taxon to this last clade, and Czatkowiella harae and Ozimek volans form a clade that is sister to the clade formed by Fuyuansaurus acutirostris and the trichotomy that includes Dinocephalosaurus orientalis. In contrast, Protorosaurus speneri is found as the sister taxon to all remaining archosauromorphs in all MPTs of analysis 4 (Fig. 36a). These remaining archosauromorphs form a polytomy in the SCT of analysis 4 consisting of Prolacerta broomi, a monophyletic Tanystropheidae, a clade composed of all OTUs previously considered as crocopodans excluding Prolacerta broomi, and the clade formed by Jesairosaurus lehmani, Pectodens zhenyuensis, and Dinocephalosaurus orientalis. Tanystropheidae is collapsed into a large polytomy in the SCT of analysis 3 and only one less inclusive clade is recovered within it, formed by Amotosaurus rotfeldensis and Augustaburiania vatagini (Fig. 35a). The tanystropheid clade in the SCT of analysis 4 is considerably more resolved (Fig. 36a). Macrocnemus bassanii and Macrocnemus fuyuanensis are recovered in a clade that is the sister group to all remaining tanystropheids. Macrocnemus obristi does not constitute a direct sister taxon to the other two Macrocnemus OTUs and is instead found as the sister taxon to all other tanystropheids. Langobardisaurus pandolfii is recovered as the sister taxon to a large polytomy within Tanystropheidae, which is formed by all other tanystropheids except the Macrocnemus OTUs. Within this polytomy less inclusive clades are formed by AMNH FARB 7206 and Tanytrachelos ahynis, and by a trichotomy Tanystropheus hydroides, Tanystropheus longobardicus, and GMPKU P 1527. These two clades form the sister groups to each other. The relationships among allokotosaurs, rhynchosaurs, and archosauriforms are consistent among all MPTs in analysis 3 (Fig. 35a). Crocopoda is formed by Allokotosauria, Rhynchosauria, Teyujagua paradoxa, and Archosauriformes as successive sister groups, and the relationships within these clades are identical to those recovered in analyses 1 and 2 . In the SCT of analysis 4, a polytomy is formed within Crocopoda by Allokotosauria, Rhynchosauria, and a clade composed of Teyujagua paradoxa and Archosauriformes (Fig. 36a). The relationships within 
3434 Allokotosauria, Rhynchosauria, and Archosauriformes in both analyses is identical to that recovered in the 3435 MPTs of the other two analyses.

3436 Five unstable OTUs were identified for analysis 3 by the iter PCR function and three RSCT were 3437 calculated. The first RSCT was generated after the exclusion a posteriori of Macrocnemus obristi and 3438 Elessaurus gondwanoccidens, which improved the resolution in the tanystropheid clade (Fig. 35b). 3439 Macrocnemus bassanii and Macrocnemus fuyuanensis are recovered in a generic clade that is the sister 3440 taxon to all remaining tanystropheids, and the clade formed by Amotosaurus rotfeldensis and 3441 Augustaburiania vatagini is found as the sister clade to the large polytomy formed by all remaining 3442 tanystropheids except the two Macrocnemus OTUs.

The second RSCT of analysis 3 additionally excludes Tanytrachelos ahynis and gains one additional node, which forms a polytomy consisting of Sclerostropheus fossai, Raibliania calligarisi, and all Tanystropheus OTUs except "Tanystropheus antiquus" (Fig. 35c).

RSCT 3 of analysis 3 is formed after the additional a posteriori pruning of Tanystropheus "conspicuus" and Raibliania calligarisi. In this RSCT, in addition to the previous improvements, Sclerostropheus fossai is recovered as the sister taxon to a trichotomy formed by Tanystropheus longobardicus, Tanystropheus hydroides, and GMPKU P 1527 (Fig. 35d).

Only two unstable OTUs were identified by the iter PCR function for analysis 4, resulting in a single RSCT for this analysis (Fig. 36b). The relationships among non-saurian diapsids are resolved after the $a$ posteriori exclusion of Orovenator mayorum. In the resulting RSCT Claudiosaurus germaini is found as the sister taxon to Sauria and a clade composed of Acerosodontosaurus piveteaui and Youngina capensis. The a posteriori exclusion of Elessaurus gondwanoccidens results in the recovery of an additional clade within Tanystropheidae, which is composed of Augustaburiania vatagini, Amotosaurus rotfeldensis, Ozimek volans, Sclerostropheus fossai, and Raibliania calligarisi as successive sister taxa. The position of Raibliania calligarisi is noteworthy, since this taxon is generally considered to be closely related to Tanystropheus longobardicus (Dalla Vecchia 2020) and is recovered in a clade with Tanystropheus longobardicus and Tanystropheus hydroides in the SCTs of analyses 1, 2, and 3.

We tested several relevant alternative topologies with our data matrix by performing iterations of analysis 4 with specific constraints applied to the tree topology. Thus, we tested how many additional steps are needed to either recover alternative clades or force an OTU outside a clade recovered compared to the unconstrained iteration of analysis 4 (Fig. 36a). We found that 14 additional steps were required to 
3464 achieve monophyly for "Protorosauria" (constraining a monophyletic group exclusively composed of the

3465 following clades and OTUs recovered in the unconstrained iteration of analysis 4: Tanystropheidae + 3466 Dinocephalosauridae + Jesairosaurus lehmani + Protorosaurus speneri + Prolacerta broomi). One 3467 additional step is required to recover a clade composed exclusively of Dinocephalosauridae and 3468 Tanystropheidae. We also tested several ingroup relationships of Tanystropheidae and found that one 3469 additional step was required to recover monophyly for Macrocnemus spp. (i.e., Macrocnemus obristi in 3470 an exclusive clade with Macrocnemus bassanii and Macrocnemus fuyuanensis) with the three 3471 Macrocnemus OTUs forming a trichotomy. Langobardisaurus pandolfii and Tanytrachelos ahynis were 3472 recovered as sister taxa by Pritchard et al. (2015), with AMNH FARB 7206 being incorporated as part of 3473 the Tanytrachelos OTU therein. For analysis 4 of our data matrix two additional steps were required to 3474 recover Langobardisaurus pandolfii in a clade exclusively with Tanytrachelos ahynis and AMNH FARB 3475 7206. It required one additional step to force Fuyuansaurus acutirostris, a taxon previously considered to 3476 possess several features typically not exhibited in tanystropheids (Fraser et al. 2013), outside 3477 Tanystropheidae, and three additional steps to recover the enigmatic Ozimek volans outside 3478 Tanystropheidae. The extreme elongation of the neck and adaptations to an aquatic lifestyle shared by 3479 Dinocephalosaurus orientalis and Tanystropheus spp. have widely been considered a striking convergence 3480 (Li et al. 2004) and six additional steps are required to recover Dinocephalosaurus orientalis and 3481 Tanystropheus spp. as sister taxa.

3482 Clade definitions and synapomorphies

3483 Analysis 4 represents the most stable analysis as is indicated by the relatively low number of MPTs 3484 for this analysis, the comparatively high resolution of its SCT, and the identification of only two unstable 3485 OTUs. The clades recovered by all MPTs of analysis 4 are defined as follows (listed unambiguous 3486 synapomorphies were common to all MPTs of analysis 4).

\section{Sauria Gauthier 1984}

Definition. The most recent common ancestor of archosaurs and lepidosaurs, and all its descendants (Gauthier et al. 1988b). \& Schneider 2008) to Recent (Crocodylus niloticus). 
3494 intertuberal plate of parabasisphenoid absent $(117: 0 \rightarrow 1)$; retroarticular process anteroposteriorly long, 3495 extending considerably posterior to the glenoid fossa (162: $1 \rightarrow 2$ ); chevrons broaden gradually distally 3496 (223: $0 \rightarrow 2)$.

3497 Lepidosauromorpha Gauthier 1984

Definition. Sphenodon and squamates and all saurians sharing a more recent common ancestor with them than they do with crocodiles and birds (Gauthier et al. 1988a). 2010) to Recent (Varanus niloticus). $0.37(1: 0 \rightarrow 1)$; anterior projection of the anterior process of the jugal up to or posterior to the level of mid-length of the orbit (37: $0 \rightarrow 1$ ); frontals fused to one another (54: $0 \rightarrow 1$ ); anterior process of the squamosal with a continuous contact along the posterior margin of the ventral process of the postorbital and contacting the jugal (64: $0 \rightarrow 1)$; supratemporal absent $(73: 1 \rightarrow 2)$; quadratojugal absent or fused to quadrate $(87: 1 \rightarrow 0)$; broad palatine, forming the main component of the palate posterior to the choanae $(98: 0 \rightarrow 1)$; palatal dentition relatively large, similar size to marginal dentition $(101: 0 \rightarrow 1)$; pterygoid teeth present in three fields (T2, T3a and T3b) (102: $0 \rightarrow 1$ ); splenial absent (143: $0 \rightarrow 1$ ); absence of a posterodorsal process of the dentary $(148: 1 \rightarrow 0)$; presence of a posterocentral process of the dentary (149: $0 \rightarrow 1$ ); atlas centrum fused to axial intercentrum (183: $0 \rightarrow 1$ ); transverse processes of caudal vertebrae distinctly angled posterolaterally from base $(221: 0 \rightarrow 1)$; chevrons of mid-caudal vertebrae with anterior curvature $(222: 0 \rightarrow 1)$; ectepicondylar region of humerus with foramen $(247: 1 \rightarrow 0)$; anteroventral process of ilium draping across anterior surface of pubis (272: $0 \rightarrow 1$ ); thyroid fenestra present (273: $0 \rightarrow 1$ ); astragalus and calcaneum fused (289: $0 \rightarrow 2$ ); presence of medial plantar tubercle of metatarsal $V(304: 0 \rightarrow 1)$.

\section{Archosauromorpha Huene 1946}

Definition. Protorosaurus and all other saurians that are related more closely to Protorosaurus than to Lepidosauria (Dilkes 1998).

\& Schneider 2008) to Recent (Crocodylus niloticus). 
Unambiguous synapomorphies. Alveolar margin of maxilla concave in lateral view $(250 \rightarrow 1)$;

3523

3524

3525

3526

3527

3528

3529

3530

3531

3532

3533

3534 supratemporal fossa of parietal expanded distinctly medially, resulting in a mediolaterally narrow parietal table (83: $0 \rightarrow 1$ ); anterior half of the surangular-angular suture anteroposteriorly concave ventrally in lateral view (158: $2 \rightarrow 0)$; upturned retroarticular process $(163: 0 \rightarrow 1)$; diapophysis and parapophysis of anterior to middle cervical postaxial vertebrae on different processes and nearly touching $(188: 0 \rightarrow 2)$; anterior margin of the neural spine of anterior and middle postaxial cervical vertebrae anterodorsally inclined at an angle of more than 60 degrees from the horizontal plane (194: $0 \rightarrow 1$ ); long transverse processes of middle dorsals (207: $0 \rightarrow 1$ ); presence of a shallow fossa lateral to the base of the neural spine of dorsal vertebrae $(209: 0 \rightarrow 1)$; coracoid moderately expanded posteriorly in lateral view (232: 2

$\rightarrow 1$ ); proximal half of interclavicle subtriangular or diamond-shaped (237: $1 \rightarrow 0$ ); torsion between proximal and distal ends of humerus 35 degrees or less (241: $0 \rightarrow 1$ ); entepicondylar foramen of humerus absent $(246: 0 \rightarrow 1)$.

\section{Unnamed clade (Prolacerta broomi + Jesairosaurus lehmani + Dinocephalosauridae + Tanystropheidae} + Allokotosauria + Rhynchosauria + Teyujagua paradoxa + Archosauriformes)

Unambiguous synapomorphies. Postnarial process of premaxilla well-developed, forming most of the ventral border of the external naris or excludes the maxilla from participation in the external naris but process does not contact prefrontal $(9: 0 \rightarrow 2)$; maxilla with abruptly ending dorsal apex and a concave posterior margin (20: $0 \rightarrow 1$ ); supraglenoid foramen of the scapula absent (231: $1 \rightarrow 0$ ); anterior margin of interclavicle with a median notch $(236: 0 \rightarrow 1)$; metatarsal $V$ with a smooth curved proximal lateral margin (302: $1 \rightarrow 0)$.

\section{Unnamed clade (Jesairosaurus + Dinocephalosauridae)}

Unambiguous synapomorphies. Anterior process of the squamosal with a continuous contact along the posterior margin of the ventral process of the postorbital and contacting the jugal $(64: 0 \rightarrow 1)$; length of the postacetabular process of the ilium versus anteroposterior length of the acetabulum between 0.50 and $0.71(267: 3 \rightarrow 0)$; tibia longer than femur $(285: 0 \rightarrow 1)$.

\section{Dinocephalosauridae new clade}

Definition. The most recent common ancestor of Pectodens zhenyuensis and Dinocephalosaurus orientalis and all of its descendants (node-based). A node-based definition of the new Dinocephalosauridae clade is preferred here because of the uncertain phylogenetic position of Jesairosaurus lehmani. 
3552

3553

3554

3555

3556

3557

3558

3559

3560

3561

3562

3563

3564

3565

3566

3567

3568

3569

3570

3571

3572

3573

3574

3575

3576

3577

3578

3579

Temporal range. Anisian (Middle Triassic; Sun et al. 2016).

Unambiguous synapomorphies. Absence of a posterior process of the jugal (42: $0 \rightarrow 1$ ); glenoid fossa considerably ventrally displaced compared to the tooth row (161: $0 \rightarrow 1$ ); postaxial anterior or middle cervical neural spine considerably shorter than the posterior articular surface of the centrum (178: $0 / 1 \rightarrow 2)$; presence of between 11 and 13 cervical vertebrae $(195: 1 \rightarrow 2)$; anterior free-ending process of anterior cervical ribs present and long, extending anterior to the prezygapophyses of the corresponding vertebra when in articulation $(200: 1 \rightarrow 2)$.

\section{Tanystropheidae Camp 1945}

Definition. The most recent common ancestor of Macrocnemus, Tanystropheus, and Langobardisaurus and all of its descendants (Dilkes 1998).

Temporal range. Induan-Olenekian (Early Triassic, Elessaurus gondwanoccidens; De Oliveira et al. 2020) to late Norian (late Late Triassic, Sclerostropheus fossai; Rigo et al. 2009; Tackett \& Tintori 2019).

Unambiguous synapomorphies. Dorsoventral height at the level of the anterior tip of the maxilla versus dorsoventral height at the level of the anterior border of the orbit between 0.20 and $0.27(2: 1 \rightarrow 0)$; prenarial process of premaxilla absent or incipient $(7: 2 \rightarrow 0)$; straight anterior part of the dorsal margin of the maxilla $(21: 0 \rightarrow 1)$; supratemporal absent $(73: 1 \rightarrow 2)$; distal margin of anterior and middle cervical postaxial neural spines completely straight along anteroposterior length in lateral view $(179: 0 \rightarrow 1)$; anterior to middle postaxial cervical neural spines with distal expansion restricted to the distal end of the neural spine (=spine table) (180: $0 \rightarrow 2$ ); neural spine of axis expanded anterodorsally in lateral view (186: $0 \rightarrow 1$ ); anterior margin of the neural spine of anterior and middle postaxial cervical vertebrae anterodorsally inclined at an angle of less than 60 degrees from the horizontal plane (194: $1 \rightarrow 2)$; height versus maximum anteroposterior length of anterior caudal neural spine between 0.42-0.83 (220: $1 \rightarrow 0)$; presence of a large fenestra between scapula and coracoid (scapulocoracoidal fenestra) (227: $0 \rightarrow 2$ ); length of the longest metacarpal versus length of the longest metatarsal between 0.36 and 0.41 (258: 2/3 $\rightarrow 1)$; thyroid fenestra present $(273: 0 \rightarrow 1)$; length of metatarsal I versus metatarsal III between 0.54 and $0.63(297: 1 \rightarrow 2$ ); length of metatarsal IV versus the proximodistal length of metatarsal $V$ between 3.65 and $5.15(305: 1 \rightarrow 3)$.

\section{Macrocnemus bassanii + Macrocnemus fuyuanensis}


Unambiguous synapomorphies. Pineal foramen of the parietal absent $(77: 0 \rightarrow 2)$; presence of

3581

3582

3583

3584

3585

3586

3587

3588

3589

3590

3591

3592

3593

3594

3595

3596

3597

3598

3599

3600

3601

3602

3603

3604

3605

3606

teeth on the lateral ramus of the pterygoid (106: $0 \rightarrow 1$ ); presence of a medial plantar tubercle on metatarsal V $(304: 0 \rightarrow 1)$.

Unnamed clade (Macrocnemus obristi + Langobardisaurus pandolfii + Fuyuansaurus acutirostris + Ozimek volans + Elessaurus gondwanoccidens + Amotosaurus rotfeldensis + Augustaburiania vatagini + Raibliania vatagini + Sclerostropheus fossai + Tanytrachelos ahynis + AMNH FARB 7206 + Tanystropheus spp.)

Unambiguous synapomorphies. There are no unambiguous synapomorphies that are shared by all MPTs for this node.

Unnamed clade (Langobardisaurus pandolfii + Fuyuansaurus acutirostris + Ozimek volans + Elessaurus gondwanoccidens + Amotosaurus rotfeldensis + Augustaburiania vatagini + Raibliania vatagini + Sclerostropheus fossai + Tanytrachelos ahynis + AMNH FARB 7206 + Tanystropheus spp.)

Unambiguous synapomorphies. Length of metatarsal IV versus length of metatarsal III between 1.03 and 1.08 (299: $2 \rightarrow 1$ ); metatarsal V with an abruptly medially flexed proximal process $(300: 2 \rightarrow 1)$; phalanx $V-1$ is metatarsal-like, considerably longer than other non-ungual phalanges (306: $0 \rightarrow 1$ ).

Unnamed clade (Fuyuansaurus acutirostris + Ozimek volans + Elessaurus gondwanoccidens + Amotosaurus rotfeldensis + Augustaburiania vatagini + Raibliania vatagini + Sclerostropheus fossai + Tanytrachelos ahynis + AMNH FARB 7206 + Tanystropheus spp.)

Unambiguous synapomorphies. Concave anterior part of the dorsal margin of the maxilla (21: 1

$\rightarrow 2$ ); not upturned retroarticular process (163: $1 \rightarrow 0$ ); epipophyses of cervical vertebrae overhanging the postzygapophysis posteriorly $(191: 0 \rightarrow 1)$; absence of interclavicle $(234: 0 \rightarrow 1)$; absence of posterior groove on astragalus (290: $0 \rightarrow 1$ ).

Unnamed clade (Tanytrachelos ahynis + AMNH FARB 7206 + Tanystropheus spp.)

Unambiguous synapomorphies. Glenoid fossa considerably ventrally displaced compared to the tooth row $(161: 0 \rightarrow 1)$; length of the postacetabular process of the ilium versus anteroposterior length of the acetabulum between 0.98 and $1.14(267: 3 \rightarrow 2)$.

Unnamed clade (Tanytrachelos ahynis + AMNH FARB 7206)

PeerJ reviewing PDF | (2020:08:51657:1:1:NEW 28 Feb 2021) 
Unambiguous synapomorphies. Procoelous presacral vertebrae (181: $2 \rightarrow 1)$; cervical ribs short,

3608

3609

3610

3611

3612

3613

3614

3615

3616

3617

3618

3619

3620

3621

3622

3623

3624

3625

3626

3627

3628

3629

3630

3631

3632

3633

3634

being less than two times the length of its respective vertebra, and shaft parallel to the neck $(199: 2 \rightarrow 1)$; preacetabular process of the ilium present and longer than two thirds of its height $(264: 1 \rightarrow 2)$.

\section{Tanystropheus spp. Meyer 1852}

Unambiguous synapomorphies. Postaxial anterior or middle cervical neural spines are only present at the anterior and posterior ends of the vertebrae but are completely or virtually lost at their anteroposterior midpoints (178: $2 \rightarrow 3$ ); lengths of the fourth or fifth cervical centra versus the heights of their anterior articular surfaces between 14.58 and 15.58 or between 17.08 and 18.67 (187: $1 \rightarrow 2 / 3$ ); total length of the humerus versus the total length of the femur between 0.84 and $0.91(248: 3 / 4 \rightarrow 2)$.

\section{Crocopoda Ezcurra 2016}

Definition. All taxa more closely related to Azendohsaurus madagaskarensis, Trilophosaurus buettneri, Rhynchosaurus articeps and Proterosuchus fergusi than to Protorosaurus speneri or Tanystropheus longobardicus (Ezcurra 2016).

Temporal range. Wuchiapingian (late Permian, Eorasaurus olsoni; Ezcurra et al. 2014) to Recent (Crocodylus niloticus).

Unambiguous synapomorphies. Alveolar margin of maxilla straight or convex in lateral view (25: $1 \rightarrow 0 / 2$; ; presence of a slightly elevated orbital rim formed by the margin of the jugal and/or postorbital (48: $0 \rightarrow 1$ ); roughly transverse orientation of the frontal-parietal contact in dorsal view (56: $1 \rightarrow 0$ ); posterolateral process of the parietal dorsoventrally deep, being plate-like in occipital view and subequal to the height of the supraoccipital $(80: 0 \rightarrow 1)$; height at the third alveolus of the dentary (or directly posterior to the tapering anterior end of the dentary in taxa with an anteriorly edentulous dentary) versus length of the alveolar margin (including edentulous anterior end if present) between 0.15 and 0.24 (144: $1 \rightarrow 2$ ); presence of hyposphene-hypantrum accessory intervertebral articulation in middle-posterior dorsals $(208: 0 \rightarrow 1)$.

\section{Allokotosauria Nesbitt, Flynn, Pritchard, Parrish, Ranivoharimanana \& Wyss 2015}

Definition. The least-inclusive clade containing Azendohsaurus madagaskarensis and Trilophosaurus buettneri but not Tanystropheus longobardicus, Proterosuchus fergusi, Protorosaurus speneri or Rhynchosaurus articeps (Nesbitt et al. 2015).

PeerJ reviewing PDF | (2020:08:51657:1:1:NEW 28 Feb 2021) 

2008) to Revueltian (middle Norian, Late Triassic, Trilophosaurus phasmalophos; Kligman et al. 2020). orbital margin of the prefrontal $(46: 0 \rightarrow 1)$; medial process of the squamosal long, forming entirely or almost entirely the posterior border of the supratemporal fenestra (70: $0 \rightarrow 1$ ); supratemporal absent

(73: $1 \rightarrow 2$ ); presence of a posteriorly hooked dorsal end of the quadrate in lateral view $(91: 0 \rightarrow 1)$; vomerine teeth relatively large, similar in size to the marginal dentition $(97: 0 \rightarrow 1)$; palatine teeth relatively large, similar in size to marginal dentition $(101: 0 \rightarrow 1)$; teeth on the ventral surface of the anterior ramus of the pterygoid relatively large, similar in size to marginal dentition (105: $0 \rightarrow 1)$; dentary ventrally curved or deflected at its anterior end (145: $0 \rightarrow 2$ ); retroarticular process anteroposteriorly short, being poorly developed posteriorly to the glenoid fossa (162: $2 \rightarrow 1$ ); lateral (=fibular) condyle of the femur projecting distally distinctly beyond medial (=tibial) condyle (282: $1 \rightarrow 0$ ); well-developed ventral tubercles of the pedal unguals, extended ventral to the articular portion of the ungual (307: $0 \rightarrow$ 1).

\section{Unnamed clade (Trilophosaurus buettneri + Azendohsaurus madagaskarensis)} ventrally deflected from the main axis of the alveolar margin (24: $0 \rightarrow 1$ ); postfrontal-frontal suture distinctly posteromedially inclined by a medial process of the postfrontal, resulting in posteriorly strongly narrowed frontal (57: $0 \rightarrow 1$ ); opisthotic and exoccipital fully fused (127: $0 \rightarrow 1$ ); paroccipital process of the opisthotic extends laterally or slightly posterolaterally (130: $1 \rightarrow 0)$; multiple teeth of the marginal dentition with distinctly mesiodistally expanded crowns above the root (170: $0 \rightarrow 1)$; second sacral rib does not bifurcate distally $(216: 1 \rightarrow 0)$; minimum anteroposterior length of the scapula less than a quarter of its proximodistal length (230: $1 \rightarrow 2$ ); presence of an olecranon process of the ulna (249: $0 \rightarrow 1$ ); presence of a lateral tuber on the proximal part of the ulna (250: $0 \rightarrow 1$ ); length of the postacetabular process of the ilium versus anteroposterior length of the acetabulum between 0.88 and 0.91 (267: $2 \rightarrow$ 1); proximal articular surface of the femur well-ossified, being flat or convex (279: $1 \rightarrow 0$ ); attachment of the caudifemoralis musculature on the posterior surface of the femur crest-like and with intertrochanteric 
Definition. All taxa more closely related to Rhynchosaurus articeps than to Trilophosaurus

3665

3666

3667

3668

3669

3670

3671

3672

3673

3674

3675

3676

3677

3678

3679

3680

3681

3682

3683

3684

3685

3686

3687

3688

3689

3690

3691 buettneri, Prolacerta broomi or Crocodylus niloticus (Ezcurra 2016).

Temporal range. Induan-early Olenekian (Early Triassic, Noteosuchus colletti; Rubidge 2005) to early Norian (Late Triassic, Teyumbaita sulcognathus; Langer et al., 2007; Montefeltro et al. 2010).

Unambiguous synapomorphies. Lateral surface of the nasal meets entire dorsoventral height of medial surface of supra-alveolar portion of maxilla $(34: 0 \rightarrow 1)$; dorsal surface of frontals covered by shallow or deep pits across surface and/or low ridges $(44: 0 \rightarrow 1)$; depression with deep pits on the dorsal surface of the postfrontal (59: $0 \rightarrow 1$ ); supratemporal present as broad element (73: $1 \rightarrow 0$ ); parietals fused with loss of suture $(74: 0 \rightarrow 1)$; fossa on the opisthotic immediately lateral to the foramen magnum $(132: 0 \rightarrow 1)$; presence of multiple zahnreihen in maxilla and dentary (164: $0 \rightarrow 1$ ); crown base of the maxillary teeth circular in shape (174: $1 \rightarrow 0)$; main axis of the postacetabular process of the ilium posterodorsally orientated in lateral or medial view $(268: 1 \rightarrow 0)$.

\section{Unnamed clade (Howesia browni + Eohyosaurus wolvaardti)}

Unambiguous synapomorphies. Presence of an anguli oris crest (27: $0 \rightarrow 1)$; supratemporal fossa of the parietal well-exposed in dorsal view and mainly dorsally or dorsolaterally facing (82: $1 \rightarrow 0$ ); supratemporal fossa of parietal expanded distinctly medially and only separated from counterpart by a sagittal crest running along the midline of the parietal $(83: 1 \rightarrow 2)$.

\section{Unnamed clade (Teyujagua paradoxa + Archosauriformes)}

Unambiguous synapomorphies. Jugal bulges ventrolaterally at the point where its three processes meet $(39: 0 \rightarrow 1)$; ventral process of the postorbital ends much higher than the ventral border of the orbit $(61: 1 \rightarrow 0)$; supratemporal fossa restricted to the lateral edge of the parietal, resulting in a broad, flat parietal table (83: $1 \rightarrow 0$ ); posttemporal fenestra absent or developed as a foramen or very narrow slit $(136: 0 \rightarrow 2)$; presence of an external mandibular fenestra $(152: 0 \rightarrow 1)$; serrations distinctly present on the distal margin of maxillary/dentary tooth crowns and usually apically restricted; low or absent on the mesial margin $(172: 0 \rightarrow 1)$.

\section{Archosauriformes Gauthier, Kluge \& Rowe 1988b}

Definition. The least inclusive clade containing Crocodylus niloticus and Proterosuchus fergusi (Nesbitt 2011).

PeerJ reviewing PDF | (2020:08:51657:1:1:NEW 28 Feb 2021) 

to Recent (Crocodylus niloticus).

Unambiguous synapomorphies. Rostrum dorsoventrally taller than transversely broad at the level

3695

3696

3697

3698

3699

3700

3701

3702

3703

3704

3705

3706

3707

3708

3709

3710

3711

3712

3713

3714

3715

3716

3717

3718

3719

3720

3721

of the anterior border of the orbit $(3: 0 \rightarrow 1)$; presence of an antorbital fenestra $(22: 0 \rightarrow 1)$; lacrimal contacts nasal but does not reach naris $(35: 2 \rightarrow 1)$; pineal foramen of the parietal absent $(77: 1 \rightarrow 2)$; postparietals sheet-like and not much narrower than the supraoccipital or small and splint-like (84: $2 \rightarrow$ $0 / 1$ ); tooth bearing portion of the dentary dorsally curved for all or most of its anteroposterior length $(145: 0 \rightarrow 1)$; presence of a posterocentral process of the dentary (149: $0 \rightarrow 1$ ); diapophysis and parapophysis of anterior to middle postaxial cervical vertebrae on different processes and well-separated $(188: 2 \rightarrow 1)$.

\section{Proterosuchus spp. Broom 1903}

Unambiguous synapomorphies. Strongly downturned main body of the premaxilla $(5: 1 \rightarrow 2)$; length of the posterior process of the jugal versus the height of its base between 5.29 and 5.84 (43: 1/2 $\rightarrow 3$ ); posterior process of the postorbital extends close to or beyond the level of the posterior margin of the supratemporal fenestrae $(60: 0 \rightarrow 1)$; presence of teeth on the lateral ramus of the pterygoid (106: 0 $\rightarrow 1)$.

\section{Unnamed clade (Erythrosuchus africanus + Euparkeria capensis)}

Unambiguous synapomorphies. Dorsoventral height at the level of the anterior tip of the maxilla versus dorsoventral height at the level of the anterior border of the orbit between 0.56 and $0.78(2: 1 \rightarrow$ 2); the anterior process of the jugal is dorsoventrally expanded and partially covers the lateral surface of the posterior process of the maxilla (38: $0 \rightarrow 1)$; supratemporal absent $(73: 1 \rightarrow 2)$; anterior process of the quadratojugal distinctly present, in which the lower temporal bar is complete, but the process terminates well posterior to the base of the posterior process of the jugal (88: $0 / 1 \rightarrow 2)$; paroccipital process of the opisthotic extends laterally or slightly posterolaterally $(130: 1 \rightarrow 0)$; lateral shelf of the surangular present as a laterally or ventrolaterally projecting shelf with a lateral edge $(155: 1 \rightarrow 2)$; serrations distinctly present on both margins of maxillary/dentary tooth crowns (172: $1 \rightarrow 2)$; middle dorsal ribs with a dichocephalous proximal end $(214: 1 \rightarrow 0)$; second sacral rib does not bifurcate distally (216: $1 \rightarrow 0$ ); scapulacoracoid with a distinct notch present on the anterior margin at the level of the suture between both bones $(227: 0 \rightarrow 1)$; minimum anteroposterior length of the scapula less than a quarter of its proximodistal length (230: $1 \rightarrow 2)$; coracoid unexpanded posteriorly in lateral view (232: 1

PeerJ reviewing PDF | (2020:08:51657:1:1:NEW 28 Feb 2021) 
$3722 \rightarrow 0$ ); approximately straight-sided shape of the preacetabular process of the ilium with a distinct angle

3723 between the anterior and dorsal margins $(265: 0 \rightarrow 1)$; dorsalmost margin of the acetabulum on the ilium

3724 is unsculptured $(271: 1 \rightarrow 0)$; presence of a pubic apron $(275: 0 \rightarrow 1)$; pedal centrale absent as a separate 3725 ossification (291: $0 \rightarrow 1$ ); absence of both distal tarsals 1 and 2 (292: $0 \rightarrow 2$ ); absence of a perforating

3726 foramen between astragalus and calcaneum $(295: 0 \rightarrow 1)$; absence of a concave gap separating the dorsal 3727 prominence from the proximo-medial surface in metatarsal $\mathrm{V}(301: 1 \rightarrow 0)$.

3728 Discussion

3729 The resolution of the SCTs of analyses 3 and 4, which employ ratio characters and treat relevant 3730 characters as ordered, is distinctly higher than those of analyses 1 and 2 and are calculated from fewer 3731 MPTs (Figs. 33-36). The topologies of the four analyses mostly correspond with each other, although 3732 several noteworthy differences occur. These relevant discrepancies between the analyses are discussed 3733 below for each corresponding OTU or clade. The results of our analyses broadly correspond to those of 3734 previous investigations of non-archosauriform archosauromorph interrelationships (e.g., Butler et al. 3735 2015; Ezcurra 2016; Ezcurra et al. 2016; Nesbitt et al. 2015; Pinheiro et al. 2019; Pritchard et al. 2015; 3736 Pritchard \& Sues 2019). Archosauromorpha is comprised of the OTUs that have widely been accepted as 3737 members of this clade in the SCTs of all four analyses. Despite a larger sample size of "protorosaurs" 3738 compared to previous analyses, particularly through the inclusion of recently described and relatively 3739 completely known Chinese "protorosaurs", as well as several tanystropheid taxa that were previously not 3740 widely considered for phylogenetic analyses (e.g., Sclerostropheus fossai, Ozimek volans, Raibliania 3741 calligarisi), all SCTs of our analyses still find "Protorosauria" to be paraphyletic (Figs. 33-36), corroborating 3742 the results of recent analyses (e.g., Ezcurra 2016; Nesbitt et al. 2015; Pritchard et al. 2015; Pritchard \& 3743 Sues 2019). Our analysis also corresponds to previous analyses in finding a monophyletic Rhynchosauria, 3744 Allokotosauria, and Archosauriformes, as well as in recovering Teyujagua paradoxa as the closest related 3745 taxon to Archosauriformes among our sampled OTUs. However, our results contradict with previous 3746 studies in the position of Prolacerta broomi, which is consistently found to be considerably more distantly 3747 related to Archosauriformes than previously considered. Furthermore, we recognize a new non3748 archosauriform archosaurmorph clade, which in all SCTs includes at least the Chinese taxa Pectodens 3749 zhenyuensis and Dinocephalosaurus orientalis. Below, the results are discussed in detail for each relevant 3750 taxon and clade.

3751 Tree resolution at the base of Archosauromorpha and the influence of ratio and ordered characters 
Although some minor differences are present, the SCT topologies for the non-"protorosaurian"

3753 OTUs, namely all non-archosauromorphs and all previously recognized crocopods except for Prolacerta

3754

3755

3756

3757

3758

3759

3760

3761

3762

3763

3764

3765

3766

3767

3768

3769

3770

3771

3772

3773

3774

3775

3776

3777

3778

3779

3780

3781

3782 broomi (rhynchosaurs, allokotosaurs, Teyujagua paradoxa, and archosauriforms) do not exhibit consistent differences between the SCTs of analyses 3 and 4 and those of analyses 1 and 2 (Figs. 33a36a). This indicates that their relative positions are quite stable for our data matrix, which is also supported by relatively high branch support values for Lepidosauromorpha, Archosauromorpha, and the relationships between and within rhynchosaurs, allokotosaurs, Teyujagua paradoxa, and Archosauriformes. The main difference between the results of analysis 1 and 2 compared to 3 and 4 is found in the resolution at the base of Archosauromorpha with the taxa that have previously been considered as "protorosaurs". In analyses 3 and 4 Tanystropheidae and Dinocephalosauridae are recovered as monophyletic clades (Figs. 35a-36a), whereas in analyses 1 and 2 these clades are collapsed and a large polytomy is formed by their OTUs, as well as Protorosaurus speneri, Jesairosaurus lehmani, and in analysis 1 Czatkowiella harae (Figs. 33a-34a). However, RSCT 1 of analysis 1 recovers Dinocephalosauridae, and RSCT 2 and 3 recover both Dinocephalosauridae and Tanystropheidae (Fig. $33 b-d)$, albeit in somewhat different compositions relative to analysis 3 and 4 as is discussed below. RSCTs 2 and 3 of analysis 2 also recover Tanystropheidae (Fig. 34c-d), but a Dinocephalosauridae clade is not recovered since both taxa comprising this clade, Pectodens zhenyuensis and Dinocephalosaurus orientalis, were identified as unstable OTUs by the iter PCR function. The broadly similar topologies between the SCTs of analyses 3 and 4 and the RSCTs of analyses 1 and 2 suggest that the addition of ratio characters and the ordering of characters has a positive effect on successfully resolving the phylogenetic relationships among non-archosauriform archosauromorphs. However, branch support values are low for all nodes within both Tanystropheidae and Dinocephalosauridae, which is likely attributable to several OTUs with large amounts of missing data within these clades. It is noteworthy to mention that the characters that were treated as ordered in analyses 3 and 4 were also formulated with the intention to be treated as such (including many characters incorporated from Ezcurra 2016). Therefore, future analyses that would decide against the use of ordered characters should reconsider the construction of some of these characters. For instance, character 84 could be split into one character treating the absence or presence of the postparietal and another describing its shape, in which the latter character is scored as inapplicable for OTUs in which the former character is scored as absent (Brazeau 2011).

The phylogenetic position of Protorosaurus speneri 
Protorosaurus speneri has been widely considered as the sister taxon to all other

3784 archosauromorphs, although it has been recovered in a less inclusive clade with Czatkowiella harae or Aenigmastropheus parringtoni at the base of Archosauromorpha in the analyses of Borsuk-Białynicka \& Evans (2009b) and Ezcurra et al. (2014), respectively. In Ezcurra (2016), Protorosaurus speneri was recovered as the sister taxon of all archosauromorphs except for the enigmatic Aenigmastropheus parringtoni. Aenigmastropheus parringtoni is known from very fragmented remains (five cervical and dorsal vertebrae, the distal part of a right humerus, the proximal part of the right ulna, and several small fragments) and was not included in our analysis. Protorosaurus speneri was recovered as the sister taxon to all other archosauromorphs in the SCT of analysis 4 (Fig. 36a), whereas it was recovered in a polytomy at the base of Archosauromorpha in the SCTs of analyses 1 and 2 (Figs. 33a-34a). However, in analysis 1, Protorosaurus speneri also obtains the position as sister taxon to all other archosauromorphs in all RSCTs (Fig. 33b-d). In analysis 2 the relationships of Protorosaurus speneri are only resolved after the a posteriori exclusion of Jesairosaurus lehmani in RSCT 3 (Figs. 34d). Therein, Protorosaurus speneri is found as the sister taxon to all other archosauromorphs as in the RSCTs of analysis 1 and the SCT of analysis 4 . The position of Protorosaurus speneri only deviates in analysis 3 , since in this analysis Protorosaurus speneri is consistently found as the sister taxon to all archosauromorphs except for Jesairosaurus lehmani (Figs. 35a). Our analyses agree with other studies in the position of Protorosaurus speneri as an early archosauromorph that is more distantly related to archosauriforms than most, if not all, other nonarchosauriform archosauromorphs are, including the tanystropheids, dinocephalosaurids, allokotosaurs, and rhynchosaurs.

The phylogenetic position of Jesairosaurus lehmani

Jesairosaurus lehmani was originally considered as a "protorosaur" that is closely related to Tanystropheidae (Jalil 1997). More recently, Ezcurra (2016) recovered Jesairosaurus lehmani as the sister taxon to Tanystropheidae. The position of Jesairosaurus lehmani is not stable in our analyses. In the SCT of analysis 1 Jesairosaurus lehmani is found in a massive polytomy at the base of Archosauromorpha (Fig. 33a). The position of Jesairosaurus lehmani within archosauromorphs is resolved after the a posteriori exclusion of Macrocnemus obristi among Archosauromorpha and in all RSCTs of analysis 1 Jesairosaurus lehmani is recovered as the sister taxon to all archosauromorphs except for Protorosaurus speneri (Figs. 33b-d). Jesairosaurus lehmani was also found as part of a polytomy at the base of Archosauromorpha in the SCT of analysis 2 and identified as one of the unstable OTUs by the iter PCR function (Figs. 34). In most 
3814 diverging member of the clade consisting of the OTUs that form Tanystropheidae and 3815 Dinocephalosauridae in the other analyses. In all MPTs of analysis 3 Jesairosaurus lehmani is found as the 3816 sister taxon to all remaining archosauromorphs (Fig. 35a) and in all MPTs of analysis 4 Jesairosaurus 3817 lehmani forms the sister taxon to Dinocephalosauridae (Figs. 36a). The clade formed by Jesairosaurus 3818 lehmani and the dinocephalosaurids is found in a polytomy with Prolacerta broomi, Tanystropheidae, and 3819 Crocopoda. Thus, the position of Jesairosaurus lehmani as a non-crocopodan archosauromorph is 3820 corroborated, but its position among non-archosauriform Archosauromorpha remains contentious based 3821 on the results of our analyses.

3822

The phylogenetic position of Czatkowiella harae

Our data matrix is the first to incorporate Czatkowiella harae since the first description of this taxon by Borsuk-Białynicka \& Evans (2009b), in which it was found as the sister taxon to Protorosaurus speneri in a clade that formed the sister group to all other archosauromorphs. Czatkowiella harae was only included in analyses 1 and 3 in our study, since it was considered as one of the problematic taxa that were pruned a priori for analyses 2 and 4 together with the nomina dubia Tanystropheus "conspicuus" and "Tanystropheus antiquus", as outlined above. Analyses 1 and 3 both recover Czatkowiella harae within Archosauromorpha (Figs. 33 and 35). In analysis 1 Czatkowiella harae and Ozimek volans were recovered in a clade in RSCTs 2 and 3 after the exclusion a posteriori of Macrocnemus obristi and Elessaurus gondwanoccidens among Archosauromorpha and this clade formed the sister group to a clade composed of Fuyuansaurus acutirostris, Tanystropheidae, and Crocopoda (Fig. 33c-d). Czatkowiella harae and Ozimek volans also form a clade in all MPTs of analysis 3, but this clade is recovered as the sister clade to Fuyuansaurus acutirostris and Dinocephalosauridae in this analysis (Fig. 35a). This indicates that at least some of the material referred to Czatkowiella harae can be attributed to Archosauromorpha, but that the taxon cannot be confidently referred to any of the less inclusive archosauromorph clades. The inclusion of Czatkowiella harae appears to have a distinct effect on the outcome of the non-archosauriform archosauromorph relationships, since the sister taxon of Czatkowiella harae, Ozimek volans, is consistently recovered within Tanystropheidae in the analyses excluding Czatkowiella harae a priori. Therefore, as long as it cannot be corroborated that the fragmented remains of Czatkowiella harae can be attributed to a single taxon unambiguously, the inclusion of this taxon should be considered carefully, since its inclusion has the potential to influence tree topology among non-archosauriform archosauromorphs.

Dinocephalosauridae 
Dinocephalosaurus orientalis has been included in four previous phylogenetic analyses. In the

3846

3847

3848

3849

3850

3851

3852

3853

3854

3855

3856

3857

3858

3859

3860

3861

3862

3863

3864

3865

3866

3867

3868

3869

3870

3871

3872

3873

3874

3875

analysis of Rieppel et al. (2008), it formed a polytomy with drepanosaurids, tanystropheids, and Jesairosaurus lehmani. In the analysis of Liu et al. (2017), which is derived from the same character matrices as used by Rieppel et al. (2008), Dinocephalosaurus orientalis was recovered within Tanystropheidae as the sister taxon to all other included tanystropheids. Dinocephalosaurus orientalis was also included in the phylogenetic analysis of De Oliveira et al. (2020), which was based on a modification of the data matrix of Pritchard et al. (2018). In this analysis Dinocephalosaurus orientalis was recovered in a clade with Jesairosaurus lehmani that represented the sister clade to all other archosauromorphs. However, the overall resolution of this analysis was poor and both Dinocephalosaurus orientalis and Jesairosaurus lehmani were pruned a priori for the final analysis presented by De Oliveira et al. (2020). Finally, Dinocephalosaurus orientalis was also included in the data matrix of Ezcurra \& Butler (2018), which was constructed with the aim to investigate the morphological disparity of the middle Permian to early Carnian archosauromorphs rather than resolve their phylogenetic relationships. In this analysis Dinocephalosaurus orientalis was found as closely related to Tanystropheidae, forming a polytomy with Tanystropheidae and the poorly known "protorosaur" Trachelosaurus fischeri in the second and least inclusive RSCT of that analysis. The phylogenetic relationships of Pectodens zhenyuensis have not been tested previously by a dedicated data matrix, but this taxon was also included in the matrix of Ezcurra \& Butler (2018) and in the SCT and both RSCTs of this analysis it was found within Tanystropheidae in a large polytomy consisting of most included tanystropheid taxa and a clade formed by the three included Macrocnemus taxa.

In the MPTs of analysis 2 Dinocephalosaurus orientalis and Pectodens zhenyuensis are either found as closely related taxa in a clade at the base of Tanystropheidae or more deeply nested within Tanystropheidae as successive sister taxa to a clade composed of the Tanystropheus OTUs, as well as Raibliania calligarisi. The latter topology could potentially be attributed to the shared presence of aquatic adaptations in Dinocephalosaurus orientalis and Tanystropheus spp., which these genera have been considered to have acquired independently (e.g., Li et al. 2004). In the SCT of analysis 2 both taxa are found in a massive polytomy at the base of Archosauromorpha (Fig. 34a). After the a posteriori exclusion of Macrocnemus obristi among Archosauromorpha, both taxa are recovered in a large polytomic clade in RSCT 1 with taxa that are generally considered as tanystropheids (Fig. 35b). Due to their unstable position in the MPTs, both Dinocephalosaurus orientalis and Pectodens zhenyuensis were omitted a posteriori by the iter PCR function for RSCTs 2 and 3.

PeerJ reviewing PDF | (2020:08:51657:1:1:NEW 28 Feb 2021) 
In analysis 1 both Dinocephalosaurus orientalis and Pectodens zhenyuensis are part of a massive

3877

3878

3879

3880

3881

3882

3883

3884

3885

3886

3887

3888

3889

3890

3891

3892

3893

3894

3895

3896

3897

3898

3899

3900

3901

3902

3903

3904

3905

3906 polytomy at the base of Archosauromorpha in the SCT (Fig. 34a). However, after the exclusion of the unstable OTU Macrocnemus obristi, both taxa are recovered in a monophyletic clade in RSCT 1, as well as the subsequent RSCTs 2 and 3 (Fig. 34b-d). This monophyletic clade is composed of a polytomy of both taxa and "Tanystropheus antiquus" and Sclerostropheus fossai. In RSCT 3 Pectodens zhenyuensis is pruned a posteriori, and the relationships within the clade are resolved with Dinocephalosaurus orientalis forming the sister taxon to a clade composed of "Tanystropheus antiquus" and Sclerostropheus fossai. In both analyses 3 and 4 Dinocephalosaurus orientalis and Pectodens zhenyuensis form a monophyletic clade in all MPTs (Figs. 35a-36a), which in the case of analysis 3 also includes "Tanystropheus antiquus". In both analysis 3 and 4 Sclerostropheus fossai is found well-nested within Tanystropheidae. It forms the sister taxon to Tanystropheus spp. in RSCT 3 (Fig. 35 ) and in analysis 4 it is recovered as the sister taxon to Raibliania calligarisi in the RSCT (Fig. 36b).

In summary, Dinocephalosaurus orientalis and Pectodens zhenyuensis form a monophyletic clade in the majority of all MPTs of all four analyses, with some MPTs also including "Tanystropheus antiquus" and Sclerostropheus fossai in this clade. However, the taxonomic status of "Tanystropheus antiquus" is currently unclear and its current phylogenetic placement should be considered with caution. Sclerostropheus fossai is exclusively known from a single, partial cervical column, and was found within Dinocephalosauridae in the RSCTs of analysis 1 (Figs. 33b-d), but this taxon could also be referrable to Tanystropheidae based on the results of analyses 3 and 4 (Figs. 35-36). The presence of a new clade consisting of at least Dinocephalosaurus orientalis and Pectodens zhenyuensis among non-archosauriform archosauromorphs is well-supported based on our data matrix. Furthermore, the presence of more Dinocephalosaurus-like taxa has been alluded to through the description of IVPP V22788, an embryonic specimen that is very similar to Dinocephalosaurus orientalis but differs in several aspects, most notably a lower number of cervical vertebrae (Li et al. 2017b). Due to the very early ontogenetic stage of this specimen, it has not been referred to a separate taxon and it has also not been included in our analyses, since very early ontogenetic features would have likely introduced biases into the analyses. Nevertheless, the clade formed by Dinocephalosaurus orientalis and Pectodens zhenyuensis, combined with the existence of at least one more Dinocephalosaurus-like taxon, merits the erection of the new higher-level taxon Dinocephalosauridae to define this clade.

Dinocephalosauridae as recovered by the RSCTs of analysis 1 forms the sister clade to a clade composing Tanystropheidae, Crocopoda, Elessaurus gondwanoccidens, Ozimek volans, Czatkowiella 
3907 harae, and Fuyuansaurus acutirostris (Fig. 33b-d). The dinocephalosaurid clade of analysis 3 is part of a 3908 larger clade formed with Fuyuansaurus acutirostris, Ozimek volans, and Czatkowiella harae, which forms 3909 the sister group to Tanystropheidae (Fig. 35a). The sister taxon to the dinocephalosaurid clade of analysis 39104 is Jesairosaurus lehmani (Fig. 36a). Together these taxa form a clade in a polytomy that includes 3911 Prolacerta broomi, Tanystropheidae, and Crocopoda. Thus, Dinocephalosauridae is well-represented as a 3912 separate clade among non-crocopodan archosauromorphs. More taxa referrable to this clade are likely to 3913 be discovered in China and possibly in other areas in the future, and their inclusion in phylogenetic 3914 analyses will aid in determining more confidently the position of Dinocephalosauridae among non3915 archosauriform archosauromorphs.

3916 The composition and interrelationships of Tanystropheidae

The monophyly of Tanystropheidae is widely supported by previous phylogenetic analyses (e.g., 3918 Benton \& Allen 1997; Dilkes 1998; Evans 1988; Ezcurra 2016; Jalil 1997; Pritchard et al. 2015; Rieppel et al. 2003, but see Simões et al. 2018 for a notable exception) and this is corroborated by our data matrix, because a monophyletic Tanystropheidae is recovered in all analyses after the a posteriori exclusion of unstable taxa. There has been less consensus on the position of tanystropheids among nonarchosauriform archosauromorphs, as well as the referral of several enigmatic taxa to Tanystropheidae. Several recent analyses recovered tanystropheids as the sister taxon to crocopods (e.g., Ezcurra 2016 and subsequent modifications of this matrix; Nesbitt et al. 2015). However, other analyses, which did not find a monophyletic Crocopoda, recovered tanystropheids as being more closely related to archosauriforms than are either rhynchosaurs (Pritchard et al. 2015) or allokotosaurs (Pritchard \& Sues 2019). Tanystropheidae are consistently found as more closely related to archosauriforms than Protorosaurus speneri in all MPTs of all four analyses and as more distantly related to archosauriforms than Teyujagua paradoxa, rhynchosaurs, and allokotosaurs are. The position of tanystropheids relative to Prolacerta broomi, Jesairosaurus lehmani, and dinocephalosaurs differs between the analyses and in the most stable analysis, analysis 4, Tanystropheidae forms a polytomy with Prolacerta broomi, Crocopoda, and a clade composed of Jesairosaurus lehmani and Dinocephalosauridae (Fig. 36).

Fuyuansaurus acutirostris was described as a "protorosaur" of uncertain phylogenetic affinities, since it shares features with known tanystropheids such as the presence of a long neck composed of elongate cervical vertebrae and accompanying ribs, but also lacks a thyroid fenestra between the pubis and ischium, which is considered a typical tanystropheid feature (Fraser et al. 2013). Fuyuansaurus 
3938 which represents the only previous phylogenetic analysis that included this taxon. The conflicting 3939 morphology relative to (other) tanystropheids as suggested by Fraser et al. (2013) is reflected in our 3940 analyses. When the unstable OTUs Macrocnemus obristi and Elessaurus gondwanoccidens are excluded $a$ 3941 posteriori, Fuyuansaurus acutirostris is found as the sister taxon to a clade composed of Tanystropheidae 3942 and Crocopoda in analysis 1 (Fig. 33c-d). In analysis 2 Fuyuansaurus acutirostris is positioned in a large 3943 polytomy consisting of tanystropheid and dinocephalosaurid OTUs in RSCT 1 (Fig. 34b), but it is identified 3944 as an unstable taxon by the iter PCR function and excluded a posteriori in RSCTs 2 and 3 . In all MPTs of analysis 3 Fuyuansaurus acutirostris forms the sister taxon to Dinocephalosauridae (Fig. 35a), whereas in the most stable analysis, analysis 4, Fuyuansaurus is quite deeply nested within Tanystropheidae (Fig. 36a). Therefore, the affinities of Fuyuansaurus acutirostris remain somewhat equivocal, and it can only very tentatively be referred to Tanystropheidae based on the currently available morphological information.

Ozimek volans differs from known tanystropheids in its extremely gracile and elongate appendicular elements and the morphology of its pectoral girdle and it was therefore not identified as a tanystropheid in the original description by Dzik \& Sulej (2016), but rather as a "protorosaur" closely related to Sharovipteryx mirabilis. However, the recent phylogenetic analysis by Pritchard \& Sues (2019), which included many archosauromorphs as well as non-saurian diapsids, recovered Ozimek volans as the sister taxon to Langobardisaurus pandolfii and Tanytrachelos ahynis deeply nested within Tanystropheidae. The position of Ozimek volans is inconsistent in our analyses. In the SCT of analysis 1 it is found in the massive polytomy at the base of Archosauromorpha, but after the a posteriori pruning of the unstable OTUs Macrocnemus obristi and Elessaurus gondwanoccidens among archosauromorphs it is found in a clade with Czatkowiella harae in RSCTs 2 and 3 (Fig. 33). This clade forms the sister group to a clade composed of Fuyuansaurus acutirostris, Tanystropheidae, and Crocopoda. Ozimek volans is found in a similar large polytomy in the SCT of analysis 2, but it is recovered deeply nested in Tanystropheidae in a polytomy with Amotosaurus rotfeldensis and a clade composed of Tanystropheus spp. and Raibliania calligarisi in RSCTs 2 and 3 after the a posteriori pruning of Macrocnemus obristi, Sclerostropheus fossai, Elessaurus gondwanoccidens, Pectodens zhenyuensis, Fuyuansaurus acutirostris, and Dinocephalosaurus orientalis among the archosauromorph OTUs (Fig. 34). In all MPTs of analysis 3 Ozimek volans is again found in a clade with Czatkowiella harae. This clade forms the sister group to a clade composed of Fuyuansaurus acutirostris and Dinocephalosauridae (Fig. 35a). In all MPTs of analysis 4 Ozimek volans is also found deeply nested within Tanystropheidae, and it is recovered as the sister taxon to a clade 
3970 gondwanoccidens among the tanystropheids in the RSCT (Fig. 36). Thus, the inclusion of Czatkowiella 3971 harae seems to have a large effect on the position of Ozimek volans, since these OTUs form a clade in 3972 most MPTs in both analyses in which the former is included. However, only a single common unambiguous 3973 synapomorphy defines the Czatkowiella harae - Ozimek volans clade in analysis 3 (the only analysis to 3974 find this clade in all MPTs): a ratio of the length versus height in posterior dorsal vertebrae between 2.16 3975 and 2.20. Regardless of the inclusion of ratio characters and the ordering of characters, the clade formed 3976 by Czatkowiella harae and Ozimek volans is found within Archosauromorpha as quite distantly related to 3977 Archosauriformes and outside Tanystropheidae (Figs. 33 and 35). Conversely, when the problematic OTU 3978 Czatkowiella harae is excluded a priori from the analyses Ozimek volans is relatively confidently recovered 3979 as a tanystropheid in both analyses 2 and 4 (Figs. 34 and 36). Therefore, the position of Ozimek volans, 3980 and by extension possibly the position of Sharovipteryx mirabilis and other putative sharovipterygids (Dzik 3981 \& Sulej 2016; Pritchard \& Sues 2019) among non-archosauriform archosauromorphs remains uncertain. 3982 Additional morphological information on Ozimek volans, including detailed comparisons to 3983 tanystropheids, other archosauromorphs, and Triassic diapsids such as drepanosauromorphs will likely 3984 aid in a more reliable phylogenetic interpretation of this taxon. This would be particularly valuable given 3985 the peculiar morphology of Ozimek volans, because the inclusion of a putative glider into Tanystropheidae would increase their known ecomorphological disparity considerably.

Macrocnemus and Tanystropheus represent the best-known tanystropheid genera. The postcrania of both Tanystropheus hydroides and Tanystropheus longobardicus possess well-known and easily recognizable characters that are considered derived compared to Macrocnemus spp., such as the presence of 13 extremely elongated cervical vertebrae and the presence of heterotopic bones in approximately $50 \%$ of specimens preserving the proximal caudal region (Nosotti 2007; Wild 1973). Recently the cranial morphology of both Tanystropheus hydroides and Macrocnemus bassanii were revised with the aid of high-resolution micro-computed tomography, highlighting a large cranial disparity between the two (Miedema et al. 2020; Spiekman et al. 2020b). The cranial morphology of Macrocnemus bassanii shares many similarities with non-tanystropheid archosauromorphs such as Prolacerta broomi and Protorosaurus speneri, particularly in the temporal region of the skull (Miedema et al. 2020), which suggests that some of these features might be plesiomorphic to Tanystropheidae. In contrast, the skull of Tanystropheus hydroides exhibits clear specializations to an aquatic lifestyle (Spiekman et al. 2020a) and possesses many cranial characters unique among tanystropheids (e.g., the configuration of the palate, a dorsoventrally flattened rostrum, the presence of a posteriorly directed hook on the dorsal end of the 
4002 incorporate the new cranial information for these taxa in a quantitative phylogenetic analysis. Our results 4003 consistently find Macrocnemus bassanii and Macrocnemus fuyuanensis in a clade that forms the sister 4004 group to all other tanystropheids when problematic OTUs are excluded (Figs. 33-36). The poorly known 4005 Macrocnemus obristi was identified as one of the unstable OTUs by the iter PCR function in analyses 1, 2, 4006 and 3 . In analysis 4 it was recovered as the sister taxon to all remaining tanystropheids except 4007 Macrocnemus bassanii and Macrocnemus fuyuanensis. Macrocnemus obristi was found to differ from the 4008 other two Macrocnemus OTUs in the lack of a sigmoidal curvature of the femur (character 286), the ratio 4009 of the length of the pes versus the length of the tibia and fibula (character 294), and the ratio of the length 4010 of metatarsal I versus that of metatarsal III (character 297). The absence of morphological information on 4011 the skull, cervical vertebrae, or pectoral girdle for Macrocnemus obristi (Fraser \& Furrer 2013), which 4012 contain many diagnostic features among tanystropheids, might have contributed to its unstable position 4013 in the MPTs of analyses 1, 2, and 3, and could explain why it was not recovered as a direct sister taxon to 4014 the other Macrocnemus OTUs in the MPTs of analysis 4. Therefore, we consider there to be insufficient 4015 support to assign Macrocnemus obristi to a separate genus despite its aberrant position in our results. In 4016 contrast to the Macrocnemus OTUs, Tanystropheus hydroides and Tanystropheus longobardicus, the best4017 known Tanystropheus spp., are consistently found deeply nested within Tanystropheidae in all MPTs of 4018 analysis 4 and in the RSCTs of the other three analyses, suggesting a derived position of the genus within 4019 Tanystropheidae.

The position of other tanystropheids is much less stable in our analyses. Raibliania calligarisi is found as closely related to Tanystropheus spp. in the RSCTs of analyses 1 and 3 and in all MPTs of analysis 2 (Figs. 33-35), reflecting the close morphological similarity of this taxon to Tanystropheus longobardicus, but it is recovered as quite distantly related to the Tanystropheus OTUs in the RSCT of analysis 4 (Fig. 36b). Elessaurus gondwanoccidens, which is only known from a single specimen comprising a hind limb and partial pelvis and vertebral column, was identified as an unstable OTU by the iter PCR function in all analyses, and in all SCTs is either found within Tanystropheidae or in a massive polytomy at the base of Archosauromorpha (Figs. 33-36). Langobardisaurus pandolfii and Tanytrachelos ahynis were found as sister taxa within Tanystropheidae in the analyses of Pritchard \& Nesbitt (2017) and Pritchard \& Sues (2019), and a close relationship between these taxa was also recovered in all MPTs of analyses 1 and 2 position within the clade is inconsistent in the MPTs and it was therefore pruned a posteriori by the iter PCR function (Fig. 35). In all MPTs of analysis 4, Tanytrachelos ahynis forms a clade with AMNH FARB 7206 that forms the sister group to a Tanystropheus spp. clade, whereas Langobardisaurus pandolfii was found 
4034 as the sister taxon to all tanystropheids except Macrocnemus spp. (Fig. 36a). In RSCTs 2 and 3 of analysis 40353 Langobardisaurus pandolfii forms a trichotomy with AMNH FARB 7206 and a clade composed of 4036 Tanystropheus spp., Raibliania calligarisi, and Sclerostropheus fossai (excluding the last two OTUs in RSCT 4037 3) after the a posteriori exclusion of Macrocnemus obristi, Elessaurus gondwanoccidens, and 4038 Tanytrachelos ahynis (Fig. 35c-d). AMNH FARB 7206 was previously tentatively referred to Tanytrachelos 4039 ahynis by Pritchard et al. (2015) but was treated as a separate OTU here (see Overview of "protorosaur" 4040 taxa section above). AMNH FARB 7206 was only found as the direct sister taxon to Tanytrachelos ahynis 4041 in SCT of analysis 4 (Fig. 36). In the SCT of analysis 1 and all RSCTs of analysis 2 AMNH FARB 7206 was 4042 found as the sister taxon to a Langobardisaurus pandolfii - Tanytrachelos ahynis clade (Figs. 33-34). In 4043 RSCTs 2 and 3 of analysis 3 AMNH FARB 7206 forms a trichotomy with Langobardisaurus pandolfii and a 4044 clade composed of Tanystropheus spp., Raibliania calligarisi, and Sclerostropheus fossai (excluding the 4045 last two OTUs in RSCT 3) (Fig. 35c-d). Therefore, the results of our analysis are ambiguous when it comes 4046 to the referral of AMNH FARB 7206 to Tanytrachelos ahynis as was previously proposed (Pritchard et al. 4047 2015). A detailed study of AMNH FARB 7206 and other specimens from the Lockatong Formation are 4048 required to determine whether this material represents a separate taxon to Tanytrachelos ahynis.

4049 The phylogenetic position of Prolacerta broomi and the composition of Crocopoda

4050 Crocopoda is a recently erected clade that is defined as all archosauromorph taxa that are more 4051 closely related to Trilophosaurus buettneri, Azendohsaurus madagaskarensis, Rhynchosaurus articeps, 4052 and Proterosuchus fergusi than to Protorosaurus speneri and Tanystropheus longobardicus (Ezcurra 2016, 4053 but see Pritchard \& Sues 2019, Simões et al. 2018, and Spiekman et al. 2020a, which found Crocopoda to 4054 be polyphyletic). Prolacerta broomi was previously considered to be very closely related to early 4055 archosauriforms (particulary the Early Triassic proterosuchids) and has been treated as such in discussions 4056 on character trait evolution (e.g., Ezcurra \& Butler 2015a; Modesto \& Sues 2004; Pinheiro et al. 2016; 4057 Pritchard \& Sues 2019). Congruently, phylogenetic analyses that found a monophyletic Crocopoda always 4058 recovered Prolacerta broomi within this clade (e.g., Butler et al. 2019; Ezcurra \& Butler 2018; Ezcurra et 4059 al. 2017; Ezcurra et al. 2019; Maidment et al. 2020; Nesbitt et al. 2017a; Nesbitt et al. 2015; Pritchard et 4060 al. 2018; Pritchard \& Nesbitt 2017; Pritchard \& Sues 2019; Scheyer et al. 2020a; Sengupta et al. 2017; 4061 Spiekman 2018; Stocker et al. 2017). In all MPTs of analyses 1 and 2 of our data matrix Prolacerta broomi 4062 is recovered within Crocopoda as the sister taxon to a clade composed of rhynchosaurs, allokotosaurs, 4063 Teyujagua paradoxa, and Archosauriformes (Figs. 33-34). In the SCTs of both analyses 3 and 4 Prolacerta 4064 broomi is found outside Crocopoda (Figs. 35-36). In the SCT of analysis 3 Prolacerta broomi forms the 
4065 sister taxon to all archosauromorphs except Jesairosaurus lehmani and Protorosaurus speneri, and 4066 Prolacerta broomi forms a polytomy with a clade composed of Jesairosaurus lehmani and 4067 Dinocephalosauridae, Tanystropheidae, and Crocopoda in the SCT of analysis 4. Our results therefore 4068 consistently find Prolacerta broomi to be considerably more distantly related to Archosauriformes than 4069 previously considered. This reflects the strong similarity in cranial morphology that has been observed 4070 between Prolacerta broomi and Macrocnemus bassanii (Miedema et al. 2020), indicating that both taxa 4071 share features that are possibly plesiomorphic to early archosauromorphs.

4072 Teyujagua paradoxa exhibits a cranial morphology that in several ways is intermediate between non4073 archosauriform archosauromorphs and archosauriforms (Pinheiro et al. 2016; Pinheiro et al. 2019). 4074 Teyujagua paradoxa is found in a polytomy with rhynchosaurs, allokotosaurs, and archosauriforms in the 4075 SCT of analysis 2 (Fig. 34a), whereas all MPTs of the other three analyses consistently finds this OTU as 4076 the sister taxon to Archosauriformes (Figs. 33a, 35a-36a). The internal nodes of the crocopodan clades 4077 Allokotosauria, Rhynchosauria, and Archosauriformes have the same composition in all four SCTs and 4078 possess relatively high support values (Figs. 33-36), which is in congruence with results of previous 4079 analyses. However, the exact interrelationships between these clades differ slightly between the analyses. 4080 In all MPTs of analyses 1 and 3 rhynchosaurs are found to be more closely related to archosauriforms than 4081 allokotosaurs are (Figs. 33a and 35a). However, in the SCT of analysis 2 a polytomy is formed by Teyujagua 4082 paradoxa, Rhynchosauria, Allokotosauria, and Archosauriformes and in the SCT of analysis 4 a trichotomy 4083 is formed by Rhynchosauria, Allokotosauria, and a clade formed by Teyujagua paradoxa and 4084 Archosauriformes (Figs. 34a and 36a). Allokotosaurs were found to be closely related to Archosauriformes 4085 by Nesbitt et al. (2015). However, other phylogenetic analyses (e.g., Ezcurra 2016 and subsequent 4086 modifications of that matrix; Pritchard \& Nesbitt 2017; Pritchard \& Sues 2019) found rhynchosaurs to be 4087 more closely related to archosauriforms. Our results reflect this discrepancy and are inconclusive 4088 regarding the relative position of Allokotosauria and Rhynchosauria among non-archosauriform 4089 Crocopoda.

\section{Macroevolutionary implications and prospectus}

4091 The results of our analyses, which include the largest "protorosaur" sample to date, reveal that 4092 non-archosauriform archosauromorphs are more diverse than previously considered, as is highlighted by 4093 the recognition of a new clade. Dinocephalosauridae includes at least two marine taxa with extensive aquatic adaptations and extremely elongated necks (Li et al. 2017b; Rieppel et al. 2008) and represents a remarkable convergence to plesiosaurs (Sauropterygia), a group of marine reptiles that was highly 
4096 successful between the Late Triassic and Late Cretaceous. Much is currently unknown about the aquatic 4097 origins of Dinocephalosaurus orientalis and the temporal range of the Dinocephalosauridae. The skeleton 4098 of the dinocephalosaurid Pectodens zhenyuensis is poorly ossified, which could represent an aquatic 4099 adaptation but is more likely attributable to the early ontogenetic state of the only known specimen, since 4100 no other clear aquatic adaptations are present in this taxon ( $\mathrm{Li}$ et al. 2017a). The tanystropheids also 4101 include at least two genera, Tanystropheus and Tanytrachelos, that both exhibit considerable neck 4102 elongation and aquatic adaptations (Olsen 1979; Spiekman et al. 2020a). Tanystropheus hydroides, 4103 Tanystropheus longobardicus, Tanystropheus "conspicuus", and Tanystropheus "haasi" are all known from 4104 Middle Triassic marine deposits (Spiekman \& Scheyer 2019), but cervical vertebrae with strong similarities 4105 to those of Tanystropheus spp. have also been discovered in Middle Triassic fluvial sediments, indicating 4106 that certain Tanystropheus-like taxa also inhabited freshwater environments (Formoso et al. 2019). The 4107 Late Triassic Tanytrachelos ahynis is exclusively known from lacustrine sediments (Casey et al. 2007; Fraser 4108 et al. 1996). Recently it was shown that two species of Tanystropheus with a large size discrepancy 4109 exhibited niche partitioning within the marine habitat of the Besano Formation at Monte San Giorgio, 4110 since both species had evolved to exploit different food sources (Spiekman et al. 2020a). This remarkable 4111 diversity of aquatic forms, originating independently in multiple lineages, suggests that non4112 archosauriform archosauromorphs represented an important component of aquatic environments. 4113 Marine taxa are currently only known from the Middle Triassic of Europe and southern China, whereas 4114 only a single freshwater form (Tanytrachelos ahynis) has been formally described so far. Future research 4115 efforts are likely to reveal a substantial increase in the known diversity and temporal extent of aquatic non-archosauriform archosauromorphs.

The presence of tanystropheids in both South America (De Oliveira et al. 2018; De Oliveira et al. 2020) and Russia (Sennikov 2011) during the Olenekian indicates that the clade already had achieved a wide, likely near-cosmopolitan, distribution before the end of the Early Triassic. Temporally, tanystropheids represent one of the most successful non-archosaurian archosauromorph lineages, extending from at least the Early Triassic until the late Late Triassic (Sclerostropheus fossai) (Fig. 37).

4122 Tanystropheids and dinocephalosaurids had almost certainly diverged from other known 4123 archosauromorph lineages by the late Permian and non-archosauriform archosauromorphs reached high 4124 ecomorphological disparity relatively soon after the Permo-Triassic mass extinction, as is indicated by the 4125 occurrence of herbivorous rhynchosaurs and allokotosaurs in the Early Triassic (Arkhangelskii \& Sennikov 4126 2008; Ezcurra et al. 2016) and the appearance of fully marine dinocelphalosaurids by the first half of the 4127 Middle Triassic (Li et al. 2017b; Rieppel et al. 2008). Consequently, our results provide additional support 
4128 to the findings of previous phylogenetic and macroevolutionary studies (e.g., Ezcurra 2016; Ezcurra \& 4129 Butler 2018; Foth et al. 2016) that have revealed that non-archosauriform archosauromorphs formed a 4130 major component of terrestrial ecosystems during the Triassic and that their palaeoecological diversity 4131 likely exceded that of the non-archosaurian archosauriforms. Our results further indicate that the diversity 4132 of aquatic non-archosauriform archosauromorphs is likely considerably higher than previous appreciated. 4133 The inclusion of the putative glider Ozimek volans among non-archosauriform archosauromorphs, and its 4134 possible referral to Tanystropheidae, provides further support for the high ecomorphological disparity of 4135 non-archosauriform Archosauromorpha. However, additional detailed comparisons of Ozimek volans and 4136 possibly other sharovipterygids to tanystropheids and other archosauromorphs are required to test these 4137 findings more rigorously.

4138 The lack of resolution and the topological inconsistency in the interrelationships of 4139 Tanystropheidae in our analyses is attributable to the large amounts of missing data in most of the known 4140 tanystropheids. Many taxa are known from either a few isolated remains (Tanystropheus "conspicuus", 4141 Augustaburiania vatagini) or a single, partial postcranial specimen (Sclerostropheus fossai, Elessaurus 4142 gondwanoccidens, Macrocnemus obristi, Raibliania calligarisi). Largely complete and articulated 4143 skeletons, including skulls, are known for several genera (e.g., Tanystropheus, Macrocnemus, 4144 Tanytrachelos, and Langobardisaurus) but their morphology is exceedingly hard to infer due to the poor 4145 preservation and the taphonomic flattening that has affected most tanystropheid fossils. However, the 4146 recent studies of flattened specimens of Macrocnemus bassanii and Tanystropheus hydroides using high4147 resolution synchrotron microtomographic scans (Miedema et al. 2020; Spiekman et al. 2020a; Spiekman 4148 et al. 2020b) have revealed their cranial morphology in high detail, revealing a large cranial disparity 4149 between these taxa. The remarkable dentition of Langobardisaurus pandolfii, characterized by an 4150 edentulous anterior end of the snout and posterior to that tricuspid teeth and large crushing teeth in the 4151 posterior part of the jaw further indicates the high cranial diversity present among the tanystropheids 4152 (Renesto \& Dalla Vecchia 2000; Saller et al. 2013). Therefore, an increased insight into the cranial 4153 morphology of other tanystropheid taxa, which are currently very poorly understood, will surely allow us 4154 to resolve tanystropheid interrelationships more confidently and will contribute to our understanding of 4155 their ecomorphological diversity. In addition to providing a detailed analysis of the interrelationships of 4156 former "protorosaurs", the data matrix constructed for this study is intended to provide a useful resource 4157 for further phylogenetic investigations on tanystropheids, dinocephalosaurids and other non4158 archosauriform archosaurmorph groups based on future findings, and to provide a phylogenetic 4159 framework for additional macroevolutionary or palaeobiographical studies. 
4160

4161

4162

4163

4164

4165

4166

4167

4168

4169

4170

4171

4172

4173

4174

4175

4176

4177

4178

4179

4180

4181

4182

4183

4184

4185

4186

\section{Conclusion}

We provide a detailed overview of all known non-archosauriform archosauromorphs previously considered as "protorosaurs". The results of our phylogenetic analyses corroborate the polyphyly of "Protorosauria" as established by previous studies (e.g., Dilkes 1998; Ezcurra 2016; Pritchard et al. 2015) and affirm that the usage of a historical "protorosaur" clade that includes tanystropheids, dinocephalosaurids, and Prolacerta broomi should be abandoned. The use of both ratio and ordered characters has a positive result on tree resolution for our sample. The Chinese taxa Pectodens zhenyuensis and Dinocephalosaurus orientalis form a newly erected clade, Dinocephalosauridae. Jesairosaurus lehmani and Fuyuansaurus acutirostris are non-crocopodan archosauromorphs that are closely related to tanystropheids and dinocephalosaurids, but their exact position among these groups is inconsistent in our analyses. The interrelationships within Tanystropheidae are poorly resolved, which can be attributed to the poorly known cranial morphology of most tanystropheid taxa. Prolacerta broomi is recovered as the sister taxon to all other Crocopoda and is thus more distantly related to Archosauriformes than previously considered.

\section{Institutional abbreviations}

AMNH - American Museum of Natural History, New York, New York, USA

AUP - University of Aberdeen, Palaeontology collection, Aberdeen, Scotland

BP - Evolutionary Studies Institute, University of Witwatersrand, Johannesburg, South Africa

BSPG - Bayerische Staatssammlung für Paläontologie und Geologie, Munich, Germany

FMNH - Field Museum of Natural History, Chicago, Illinois, USA

GMPKU - Geological Museum of Peking University, Beijing, China

IGWuG - Institut für Geologische Wissenschaften und Geiseltalmuseum, Martin-Luther-Universität HalleWittenberg, Halle, Germany

IVPP - Institute of Vertebrate Paleontology and Paleoanthropology, Beijing, China

KUVP - Kansas University Museum of Natural History, Lawrence, Kansas, USA

LPV - Chengdu Center of the China Geological Survey, Chengdu, Sichuan, China

MCSN - Museo Cantonale di Scienze Naturali di Lugano, Lugano, Switzerland 
4187 MCSNB - Museo Civico di Scienze Naturali "E. Caffi" Bergamo, Bergamo, Italy

4188 MFSN - Museo Friulano di Scienze Naturali, Udine, Italy

4189 MGUWr - Geological Museum, Institute of Geological Sciences, University of Wrocław, Wrocław, Poland

4190 MSNM - Museo di Storia Naturale, Milan, Italy

4191 NMS - National Museums Scotland, Edinburgh, UK

4192 NHMW - Naturhistorisches Museum Wien, Vienna, Austria

4193 NMK - Naturkundemuseum im Ottoneum, Kassel, Germany

4194 OMNH - Sam Noble Oklahoma Museum of Natural History, Norman, Oklahoma, USA

4195 P - Palaeontological Collection of the Department of Geology and Palaeontology, University of Innsbruck, 4196 Innsbruck, Austria

4197 PIMUZ - Paläontologisches Institut und Museum der Universität Zürich, Zurich, Switzerland

4198 PIN - Paleontological Institute of the Russian Academy of Sciences, Moscow, Russia

4199 RC - Rubidge Collection, Wellwood, Graaff-Reinet, South Africa

4200 RCSHC - Royal College of Surgeons Hunterian Collection, London, England

4201 SAM-PK - Iziko South African Museum, Cape Town, South Africa

4202 SMNS - Staatliches Museum für Naturkunde, Stuttgart, Germany

4203 SMNK - Staatliches Museum für Naturkunde Karlsruhe, Germany

4204 TMM - Texas Memorial Museum, Austin, Texas, USA

4205 TWCMS - Sunderland Museum and Winter Gardens, Tyne \& Wear Archives \& Museums, Sunderland, 4206 England

4207 UA - University of Antananarivo, Antananarivo, Madagascar

4208 UCMP - University of California Museum of Paleontology, Berkeley, California, USA

4209 UFSM - Universidade Federal de Santa Maria, Santa Maria, Brazil

4210 UMMP - University of Michigan Museum of Paleontology, Ann Arbor, Michigan, USA 
4211

4212

4213

4214

4215

4216

4217

4218

4219

4220

4221

4222

4223

4224

4225

4226

4227

4228

4229

4230

4231

4232

4233

4234

4235

4236

4237

UMZC - University Museum of Zoology, Cambridge, England

UNIPAMPA - Universidade Federal do Pampa, São Gabriel, Brazil

UWBM - Burke Museum of Natural History and Culture, University of Washington, Seattle, Washington, USA

VMNH - Virginia Museum of Natural History, Martinsville, Virginia, USA

WMsN - LWL-Museum für Naturkunde, Westfälisches Landesmuseum mit Planetarium, Münster, Germany

YPM - Yale Peabody Museum, New Haven, Connecticut, USA

ZAR - Zarzaitine Collection, Muséum National d'Histoire Naturelle, Paris, France

ZMNH - Zhejiang Museum of Natural History, Hangzhou, China

ZPAL - Institute of Paleobiology, Polish Academy of Sciences, Warsaw, Poland

\section{Acknowledgements}

We kindly thank Mark Norell and Carl Mehling (AMNH), Kevin Padian and Pat Holroyd (UCMP), Christian Sidor and Meredith Rivin (UWBM), Claire Browning and Roger Smith (SAM-PK), Anna Paganoni (MCSNB), Bernhard Zipfel, Sifelani Jirah, and Jonah Choiniere (BP), Rudolf Stockar (MCSN), Cristiano Dal Sasso and Stefania Nosotti (MSNM), Cristina Lombardo (MPUM), Nour-Eddine Jalil (ZAR), Christian Klug and Beat Scheffold (PIMUZ), Alexander Hastings and Christina Byrd (VMNH), Rainer Schoch (SMNS), Eberhard “Dino” Frey (SMNK), Lothar Schöllmann (WMsN), Cornelia Kurz (NMK), Giuseppe Muscio, Luca Simonetto, and Fabia Dalla Vecchia (MFSN), Li Chun (IVPP), Da-Yong Jiang (GMPKU), and Olivier Rieppel and William Simpson (FMNH), for access to specimens under their care. Martín Ezcurra, Thodoris Argyriou, Gabriel Aguirre Fernandez, Dylan Bastiaans, Feiko Miedema, and Olivier Rieppel are thanked for discussions. Martín Ezcurra and Sterling Nesbitt kindly provided photographs of several specimens of Azendohsaurus madagaskarensis, Pamelaria dolichotrachela, and Proterosuchus alexanderi. TNT is made freely available by the Willi Hennig Society. Finally, we would like to thank academic editor Claudia Marsicano and reviewers Martín Ezcurra and Felipe Pinheiro for their constructive recommendations that improved the quality of this manuscript.

\section{References}


4238

4239

4240

4241

4242

4243

4244

4245

4246

4247

4248

4249

4250

4251

4252

4253

4254

4255

4256

4257

4258

4259

4260

4261

4262

4263

4264

4265

4266

4267

4268

4269

4270

4271

4272

4273

4274

4275

4276

4277

4278

4279

4280

4281

4282

4283

4284

4285
Abdala F, Hancox PJ, and Neveling J. 2005. Cynodonts from the uppermost Burgersdorp Formation, South Africa, and their bearing on the biostratigraphy and correlation of the Triassic Cynognathus Assemblage Zone. Journal of Vertebrate Paleontology 25:192-199. 10.1671/02724634(2005)025[0192:CFTUBF]2.0.CO;2

Arkhangelskii M, and Sennikov A. 2008. "Subclass Synaptosauria". In: Ivakhnenko MF, and Kurotchkin EN, eds. "Fossil vertebrates of Russia and adjacent countries: Fossil reptiles and birds Part 1". Moscow: GEOS, 224-241 (in Russian).

Arthaber Gv. 1922. Über Entwicklung, Ausbildung und Absterben der Flugsaurier. Paläontologische Zeitschrift 4:1-47.

Bartholomai A. 1979. New lizard-like reptiles from the Early Triassic of Queensland. Alcheringa 3:225-234.

Bassani F. 1886. Sui fossili e sull'eta degli schisti bituminosi triasici di Besano in Lombardia. Atti Società Italiana di scienze naturali 29:15-72.

Bennett SC. 1996. The phylogenetic position of the Pterosauria within the Archosauromorpha. Zoological Journal of the Linnean Society 118:261-308. 10.1111/j.1096-3642.1996.tb01267.x

Benton MJ. 1983. The Triassic reptile Hyperodapedon from Elgin: functional morphology and relationships. Philosophical Transactions of the Royal Society of London B, Biological Sciences 302:605-718. 10.1098/rstb.1983.0079

Benton MJ. 1984. The relationships and early evolution of the Diapsida. In: Ferguson MWJ, ed The structure, evolution, and development of reptiles London: Symposia of the Zoological Society of London 52:575-596.

Benton MJ. 1985. Classification and phylogeny of the diapsid reptiles. Zoological Journal of the Linnean Society 84:97-164. 10.1111/j.1096-3642.1985.tb01796.x

Benton MJ. 2005. Vertebrate Palaeontology. Wiley-Blackwell 3rd Edition:472 pages.

Benton MJ, and Allen JL. 1997. Boreopricea from the Lower Triassic of Russia, and the relationships of the prolacertiform reptiles. Palaeontology 40:931-953.

Benton MJ, and Walker AD. 1996. Rhombopholis, a prolacertiform reptile from the Middle Triassic of England. Palaeontology 39:763-782.

Bickelmann C, Müller J, and Reisz RR. 2009. The enigmatic diapsid Acerosodontosaurus piveteaui (Reptilia: Neodiapsida) from the Upper Permian of Madagascar and the paraphyly of "younginiform" reptiles. Canadian Journal of Earth Sciences 46:651-661. 10.1139/E09-038

Bizzarini F, and Muscio G. 1995. Un nuovo rettile (Reptilia, Prolacertiformes) dal Norico di Preone (Udine, Italia Nordorientale). Nota preliminare. Gortania - Atti del Museo Friulano di Storia Naturale 16:67-76.

Bock W. 1945. A new small reptile from the Triassic of Pennsylvania. Notulae Naturae of the Academy of Natural Sciences of Philadelphia 154:1-8.

Borsuk-Bialynicka M, and Evans SE. 2003. A basal archosauriform from the Early Triassic of Poland. Acta Palaeontologica Polonica 48.

Borsuk-Białynicka M, and Evans SE. 2009a. Cranial and mandibular osteology of the Early Triassic archosauriform Osmolskina czatkowicensis from Poland. Palaeontologia Polonica 65:235-281.

Borsuk-Białynicka M, and Evans SE. 2009b. A long-necked archosauromorph from the Early Triassic of Poland. Palaeontologia Polonica 65:203-234.

Borsuk-Białynicka M, and Lubka M. 2009. Procolophonids from the Early Triassic of Poland. Palaeontologia Polonica 65:107-144.

Borsuk-Białynicka M, and Sennikov AG. 2009. Archosauriform postcranial remains from the Early Triassic karst deposits of southern Poland. Palaeontologia Polonica 65:283-328.

Botha-Brink J, and Smith RMH. 2011. Osteohistology of the Triassic archosauromorphs Prolacerta, Proterosuchus, Euparkeria, and Erythrosuchus from the Karoo Basin of South Africa. Journal of Vertebrate Paleontology 31:1238-1254. 10.1080/02724634.2011.621797

Peer) reviewing PDF | (2020:08:51657:1:1:NEW 28 Feb 2021) 
4286

4287

4288

4289

4290

4291

4292

4293

4294

4295

4296

4297

4298

4299

4300

4301

4302

4303

4304

4305

4306

4307

4308

4309

4310

4311

4312

4313

4314

4315

4316

4317

4318

4319

4320

4321

4322

4323

4324

4325

4326

4327

4328

4329

4330

4331
Brazeau MD. 2011. Problematic character coding methods in morphology and their effects. Biological Journal of the Linnean Society 104:489-498. 10.1111/j.1095-8312.2011.01755.x

Broili F, and Fischer E. 1918. Trachelosaurus fischeri nov. gen. nov. sp. Ein neuer Saurier aus dem Buntsandstein von Bernburg. Jahrbuch der Königlich Preussischen Geologischen Landesanstalt zu Berlin 37:359-414.

Broom R. 1903. On a new reptile (Proterosuchus fergusi) from the Karoo beds of Tarkastad, South Africa. Annals of the South African Museum 4:158-164.

Broom R. 1905a. Preliminary notice of some new fossil reptiles collected by Mr. Alfred Brown at Aliwal North, South Africa. Records of the Albany Museum 1:269-275.

Broom R. 1905b. Notice of some new fossil reptiles from the Karroo beds of South Africa. Records of the Albany Museum 1:331-337.

Broom R. 1913a. Note on Mesosuchus browni, Watson, and on a new South African Triassic pseudosuchian (Euparkeria capensis). Records of the Albany Museum 2:394-396.

Broom R. 1913b. On the South-African pseudosuchian Euparkeria and allied genera. Proceedings of the Zoological Society of London 1913:619-633. 10.1111/j.1469-7998.1913.tb06148.x

Broom R. 1914. A new Thecodont Reptile. Proceedings of the Zoological Society of London:1072-1077.

Broom R. 1922. An imperfect skeleton of Youngina capensis, Broom, in the collection of the Transvaal Museum. Annals of the Transvaal Museum 8:273-276.

Broom R. 1925. On the origin of lizards. Proceedings of the Zoological Society of London 1925: 1-16. 10.1111/j.1096-3642.1925.tb03338.x

Broom R. 1937. A further contribution to our knowledge of the fossil reptiles of the Karroo. Proceedings of the Zoological Society of London 1937:299-318. 10.1111/j.1469-7998.1937.tb00011.x

Broom R, and Robinson JT. 1948. Some new fossil reptiles from the Karoo beds of South Africa. Proceedings of the Zoological Society of London 1948:392-407. 10.1111/j.10963642.1948.tb00384.x

Brown EE, Butler RJ, Ezcurra MD, Bhullar BAS, and Lautenschlager S. 2019. Endocranial anatomy and life habits of the Early Triassic archosauriform Proterosuchus fergusi. Palaeontology 63:255-282. 10.1111/pala.12454

Butler RJ, Ezcurra MD, Liu J, Sookias RB, and Sullivan C. 2019. The anatomy and phylogenetic position of the erythrosuchid archosauriform Guchengosuchus shiguaiensis from the earliest Middle Triassic of China. PeerJ 7:e6435. 10.7717/peerj.6435

Butler RJ, Ezcurra MD, Montefeltro FC, Samathi A, and Sobral G. 2015. A new species of basal rhynchosaur (Diapsida: Archosauromorpha) from the early Middle Triassic of South Africa, and the early evolution of Rhynchosauria. Zoological Journal of the Linnean Society 174:571-588.

Caldwell MW. 1995. Developmental constraints and limb evolution in Permian and extant lepidosauromorph diapsids. Journal of Vertebrate Paleontology 14:459-471. 10.1080/02724634.1995.10011572

Calzavara M, Muscio G, and Wild R. 1980. Megalancosaurus preonensis n.g., n.sp., a new reptile from the Norian of Friuli, Italy. Gortania - Atti del Museo Friulano di Storia Naturale 2:59-64.

Camp CL. 1945. Prolacerta and the protorosaurian reptiles. Part I and Part II. American Journal of Science 243:17-32, 84-101. 10.2475/ajs.243.1.17

Carrano MT, and Sampson SD. 2004. A review of coelophysoids (Dinosauria: Theropoda) from the Early Jurassic of Europe, with comments on the late history of the Coelophysoidea. Neues Jahrbuch für Geologie und Paläontologie-Monatshefte 9:537-558. 10.1127/njgpm/2004/2004/537

Carroll RL. 1981. Plesiosaur ancestors from the Upper Permian of Madagascar. Philosophical Transactions of the Royal Society of London B, Biological Sciences 293:315-383. 10.1098/rstb.1981.0079

Peer) reviewing PDF | (2020:08:51657:1:1:NEW 28 Feb 2021) 
4332

4333

4334

4335

4336

4337

4338

4339

4340

4341

4342

4343

4344

4345

4346

4347

4348

4349

4350

4351

4352

4353

4354

4355

4356

4357

4358

4359

4360

4361

4362

4363

4364

4365

4366

4367

4368

4369

4370

4371

4372

4373

4374

4375

4376

4377

4378

4379
Case EC. 1928. A cotylosaur from the Upper Triassic of western Texas. Journal of the Washington Academy of Sciences 18:177-178.

Casey MM, Fraser NC, and Kowalewski M. 2007. Quantative taphnomy of a Triassic reptile Tanytrachelos ahynis from the Cow Branch Formation, Dan River Basin, Solite Quarry, Virginia. PALAIOS 22:598611. 10.2110/palo.2006.p06-010r

Castiello M, Renesto S, and Bennett SC. 2016. The role of the forelimb in prey capture in the Late Triassic reptile Megalancosaurus (Diapsida, Drepanosauromorpha). Historical Biology 28:1090-1100. 10.1080/08912963.2015.1107552

Chatterjee S. 1980. Malerisaurus, a new eosuchian reptile from the Late Triassic of India. Philosophical Transactions of the Royal Society of London B: Biological Sciences 291:163-200.

Chatterjee S. 1986. Malerisaurus langstoni, a new diapsid reptile from the Triassic of Texas. Journal of Vertebrate Paleontology 6:297-312. 10.1080/02724634.1986.10011627

Cruickshank ARI. 1972. The proterosuchian thecodonts. In Studies in Vertebrate Evolution, by Joysey KA and Kemp TS, eds Oliver \& Boyd, Edinburgh:89-119.

Cruickshank ARI. 1979. The ankle joint in some early archosaurs. South African Journal of Science 75:168.

Cohen K, Finney S, Gibbard P, and Fan J-X. 2013. The ICS international chronostratigraphic chart. Episodes 36:199-204.

Colbert EH. 1945. The Dinosaur Book: The Ruling Reptiles and their Relatives. Man and Nature Publications Handbook.

Colbert EH. 1965. The Age of Reptiles. Norton, New York:262 p.

Colbert EH. 1987. The Triassic Reptile Prolacerta in Antartica. American Museum Novitates:1-19.

Colbert EH, and Olsen PE. 2001. A new and unusual aquatic reptile from the Lockatong Formation of New Jersey (Late Triassic, Newark Supergroup). American Museum Novitates 2001:1-24. 10.1206/0003-0082(2001)334<0001:ANAUAR>2.0.CO;2

Conrad J. 2008. Phylogeny and systematics of Squamata (Reptilia) based on morphology. Bulletin of the American Museum of Natural History 310:1-182. 10.1206/310.1

Cope ED. 1900. The crocodilians, lizards and snakes of North America. Washington: Government Printing Office.

Cowen R. 1981. Homonyms of Podopteryx. Journal of Paleontology 55:483.

Cubo J, and Jalil N-E. 2019. Bone histology of Azendohsaurus laaroussii: Implications for the evolution of thermometabolism in Archosauromorpha. Paleobiology 45:317-330. 10.1017/pab.2019.13

Currie PJ. 1980. A new younginid (Reptilia: Eosuchia) from the Upper Permian of Madagascar. Canadian Journal of Earth Sciences 17:500-511. 10.1139/e80-046

Currie PJ. 1981. The vertebrae of Youngina (Reptilia: Eosuchia). Canadian Journal of Earth Sciences 18:815818. 10.1139/e81-075

Dalla Vecchia FM. 2020. Raibliania calligarisi gen. n., sp. n., a new tanystropheid (Diapsida, Tanystropheidae) from the Upper Triassic (Carnian) of northeastern Italy. Rivista Italiana di Paleontologia e Stratigrafia 126:197-222. 10.13130/2039-4942/13041

De Oliveira TM, Oliveira D, Schultz CL, Kerber L, and Pinheiro FL. 2018. Tanystropheid archosauromorphs in the Lower Triassic of Gondwana. Acta Palaeontologica Polonica 63: 713-723. 10.4202/app.00489.2018

De Oliveira TM, Pinheiro FL, Stock Da Rosa ÁA, Dias Da Silva S, and Kerber L. 2020. A new archosauromorph from South America provides insights on the early diversification of tanystropheids. PLOS ONE 15:e0230890. 10.1371/journal.pone.0230890

DeBraga M, and Reisz RR. 1995. A new diapsid reptile from the uppermost Carboniferous (Stephanian) of Kansas. Palaeontology 38:199-212.

DeBraga M, and Rieppel O. 1997. Reptile phylogeny and the interrelationships of turtles. Zoological Journal of the Linnean Society 120:281-354. 10.1111/j.1096-3642.1997.tb01280.x

Peer) reviewing PDF | (2020:08:51657:1:1:NEW 28 Feb 2021) 
4380

4381

4382

4383

4384

4385

4386

4387

4388

4389

4390

4391

4392

4393

4394

4395

4396

4397

4398

4399

4400

4401

4402

4403

4404

4405

4406

4407

4408

4409

4410

4411

4412

4413

4414

4415

4416

4417

4418

4419

4420

4421

4422

4423

4424

4425

4426

4427

De Buffrénil V, and Mazin JM. 1989. Bone histology of Claudiosaurus germaini (Reptilia, Claudiosauridae) and the problem of pachyostosis in aquatic tetrapods. Historical Biology 2:311-322. 10.1080/08912968909386509

Demes B, and Krause DW. 2005. Suction feeding in a Triassic protorosaur? Science 308:1112-1113. 10.1126/science.308.5725.1112c

Demuth OE, Rayfield EJ, and Hutchinson JR. 2020. 3D hindlimb joint mobility of the stem-archosaur Euparkeria capensis with implications for postural evolution within Archosauria. Scientific reports 10:1-14. 10.1038/s41598-020-70175-y

Desojo JB, Ezcurra MD, and Schultz CL. 2011. An unusual new archosauriform from the Middle-Late Triassic of southern Brazil and the monophyly of Doswelliidae. Zoological Journal of the Linnean Society 161:839-871. 10.1111/j.1096-3642.2010.00655.x

Dias-da-Silva S, Pinheiro FL, Da-Rosa ÁAS, Martinelli AG, Schultz CL, Silva-Neves E, and Modesto SP. 2017. Biostratigraphic reappraisal of the Lower Triassic Sanga do Cabral Supersequence from South America, with a description of new material attributable to the parareptile genus Procolophon. Journal of South American Earth Sciences 79:281-296. 10.1016/j.jsames.2017.07.012

Dilkes DW. 1995. The rhynchosaur Howesia browni from the Lower Triassic of South Africa. Palaeontology 38:665-686.

Dilkes DW. 1998. The Early Triassic rhynchosaur Mesosuchus browni and the interrelationships of basal archosauromorph reptiles. Philosophical Transactions of the Royal Society of London B: Biological Sciences 353:501-541. 10.1098/rstb.1998.0225

Dilkes DW, and Arcucci AB. 2012. Proterochampsa barrionuevoi (Archosauriformes: Proterochampsia) from the Late Triassic (Carnian) of Argentina and a phylogenetic analysis of Proterochampsia. Palaeontology 55:853-885. 10.1111/j.1475-4983.2012.01170.x

Dilkes DW, and Sues H-D. 2009. Redescription and phylogenetic relationships of Doswellia kaltenbachi (Diapsida: Archosauriformes) from the Upper Triassic of Virginia. Journal of Vertebrate Paleontology 29:58-79. 10.1080/02724634.2009.10010362

Dutuit J. 1972. Découverte d'un dinosaure ornithischien dans le Trias supérieur de I'Atlas occidental marocain. Comptes Rendus de l'Académie des Sciences 275:2841-2844.

Dzik J, and Sulej T. 2007. A review of the early Late Triassic Krasiejów biota from Silesia, Poland. Palaeontologia Polonica:3-27.

Dzik J, and Sulej T. 2016. An early Late Triassic long-necked reptile with a bony pectoral shield and gracile appendages. Acta Palaeontologica Polonica 61:805-823. 10.4202/app.00276.2016

Edinger T. 1924. Rückenmark im Wirbelkörper! Anatomischer Anzeiger 57:515-519.

El-Toubi MR. 1949. The post-cranial osteology of the lizard, Uromastyx aegyptia (Forskål). Journal of Morphology 84:281-292. 10.1002/jmor.1050840205

Ellenberger P. 1977. Quelques précisions sur l'anatomie et la place systématique trés spéciale de Cosesaurus Aviceps. (Ladinien Su périeur de Montral, Catalogne). Cuadernos de geología Ibérica 4:169-188.

Ellenberger P. 1978. L'origine des oiseaux. Historique et méthodes nouvelles. Les problèmes des Archaeornithes. La venue au jour de Cosesaurus aviceps. Mémoires Travaux EPHE, Institut Montpellier 4:91-117.

Ellenberger $\mathrm{P}$, and De Villalta JF. 1974. Sur la présence d'un ancêtre probable des oiseaux dans le Muschelkalk supérieur de Catalogne (Espagne). Note préliminaire. Acta geológica hispánica 9:162-168.

Evans SE. 1980. The skull of a new eosuchian reptile from the Lower Jurassic of South Wales. Zoological Journal of the Linnean Society 70:203-264. 10.1111/j.1096-3642.1980.tb00852.x

Evans SE. 1981. The postcranial skeleton of the Lower Jurassic eosuchian Gephyrosaurus bridensis. Zoological Journal of the Linnean Society 73:81-116. 10.1111/j.1096-3642.1981.tb01580.x

Peer) reviewing PDF | (2020:08:51657:1:1:NEW 28 Feb 2021) 
4428

4429

4430

4431

4432

4433

4434

4435

4436

4437

4438

4439

4440

4441

4442

4443

4444

4445

4446

4447

4448

4449

4450

4451

4452

4453

4454

4455

4456

4457

4458

4459

4460

4461

4462

4463

4464

4465

4466

4467

4468

4469

4470

4471

4472

4473

4474

4475
Evans SE. 1986. The braincase of Prolacerta broomi (Reptilia, Triassic). Neues Jahrbuch für Geologie und Paläontologie Abhandlungen 173:181-200.

Evans SE. 1987. The braincase of Youngina capensis (Reptilia: Diapsida; Permian). Neues Jahrbuch für Geologie und Paläontologie Monatshefte 1987:193-203.

Evans SE. 1988. The early history and relationships of the Diapsida. In The Phylogeny and Classification of the Tetrapods, Volume 1: Amphibians, Reptiles, Birds, by Benton, M J, Clarendon Press, Oxford Systematics Association Special Volume No. 35A:221-260.

Evans SE. 2009. An early kuehneosaurid reptile from the Early Triassic of Poland. Palaeontologia Polonica 65:145-178.

Evans SE, and Borsuk-Białynicka M. 2009. A small lepidosauromorph reptile from the Early Triassic of Poland. Palaeontologia Polonica 65:179-202.

Evans SE, and Jones MEH. 2010. The origin, early history and diversification of lepidosauromorph reptiles. In: Bandyopadhyay S, ed. New aspects of Mesozoic biodiversity: Springer, 27-44.Evans SE, and Kermack K. 1994. Assemblages of small tetrapods from the Early Jurassic of Britain. In: Fraser NC, and Sues H-D, eds. In the shadow of the dinosaurs: Early Mesozoic tetrapods: Cambridge University Press, 271-283.

Evans SE, and King MS. 1993. A new specimen of Protorosaurus (Reptilia: Diapsida) from the Marl Slate (late Permian) of Britain. Proceedings of the Yorkshire Geological Society 49:229-234. 10.1144/pygs.49.3.229

Evans SE, and Van Den Heever JA. 1987. A new reptile (Reptilia: Diapsida) from the Upper Permian Daptocephalus zone of South Africa. South African Journal of Science 83:724-730.

Ewer RF. 1965. The anatomy of the thecodont reptile Euparkeria capensis Broom. Philosophical Transactions of the Royal Society B 248:379-435. 10.1098/rstb.1965.0003

Ezcurra MD. 2016. The phylogenetic relationships of basal archosauromorphs, with an emphasis on the systematics of proterosuchian archosauriforms. PeerJ 4:e1778. 10.7717/peerj.1778

Ezcurra MD, and Butler RJ. 2015a. Post-hatchling cranial ontogeny in the Early Triassic diapsid reptile Proterosuchus fergusi. Journal of Anatomy 226:387-402. 10.1111/joa.12300

Ezcurra MD, and Butler RJ. 2015b. Taxonomy of the proterosuchid archosauriforms (Diapsida: Archosauromorpha) from the earliest Triassic of South Africa, and implications for the early archosauriform radiation. Palaeontology 58:141-170. 10.1111/pala.12130

Ezcurra MD, and Butler RJ. 2018. The rise of the ruling reptiles and ecosystem recovery from the PermoTriassic mass extinction. Proceedings of the Royal Society B: Biological Sciences 285: 20180361. $10.1098 / \mathrm{rspb} .2018 .0361$

Ezcurra MD, Desojo JB, and Rauhut OWM. 2015a. Redescription and phylogenetic relationships of the proterochampsid Rhadinosuchus gracilis (Diapsida: Archosauriformes) from the early Late Triassic of southern Brazil. Ameghiniana 52:391-417. 10.5710/AMGH.28.04.2015.2867

Ezcurra MD, Fiorelli LE, Martinelli AG, Rocher S, von Baczko MB, Ezpeleta M, Taborda JR, Hechenleitner EM, Trotteyn MJ, and Desojo JB. 2017. Deep faunistic turnovers preceded the rise of dinosaurs in southwestern Pangaea. Nature ecology \& evolution 1:1477-1483. 10.1038/s41559-017-0305-5

Ezcurra MD, Gower DJ, Sennikov AG, and Butler RJ. 2019. The osteology of the holotype of the early erythrosuchid Garjainia prima (Diapsida: Archosauromorpha) from the upper Lower Triassic of European Russia. Zoological Journal of the Linnean Society 185:717-783. 10.1093/zoolinnean/zly061

Ezcurra MD, Lecuona A, and Martinelli A. 2010. A new basal archosauriform diapsid from the Lower Triassic of Argentina. Journal of Vertebrate Paleontology 30:1433-1450. 10.1080/02724634.2010.501446

Ezcurra MD, Montefeltro F, and Butler RJ. 2016. The early evolution of rhynchosaurs. Frontiers in Ecology and Evolution 3:142. 10.3389/fevo.2015.00142

Peer] reviewing PDF | (2020:08:51657:1:1:NEW 28 Feb 2021) 
4476

4477

4478

4479

4480

4481

4482

4483

4484

4485

4486

4487

4488

4489

4490

4491

4492

4493

4494

4495

4496

4497

4498

4499

4500

4501

4502

4503

4504

4505

4506

4507

4508

4509

4510

4511

4512

4513

4514

4515

4516

4517

4518

4519

4520

4521

Ezcurra MD, Nesbitt SJ, Bronzati M, Dalla Vecchia FM, Agnolin FL, Benson RB, Egli FB, Cabreira SF, Evers SW, and Gentil AR. 2020. Enigmatic dinosaur precursors bridge the gap to the origin of Pterosauria. Nature 588:445-449. 10.1038/s41586-020-3011-4

Ezcurra MD, Scheyer TM, and Butler RJ. 2014. The origin and early evolution of Sauria: reassessing the Permian saurian fossil record and the timing of the crocodile-lizard divergence. PLOS ONE 9:e89165. 10.1371/journal.pone.0089165

Ezcurra MD, Velozo P, Meneghel M, and Piñeiro G. 2015b. Early archosauromorph remains from the Permo-Triassic Buena Vista Formation of north-eastern Uruguay. PeerJ 3:e776. 10.7717/peerj.776

Feduccia A. 1996. The origin and evolution of Birds. Yale University Press New Haven and London.

Feduccia A, and Wild R. 1993. Birdlike Characters in the Triassic Archosaur Megalancosaurus. Naturwissenschaften 80:564-566.

Feist-Burkhardt S, Götz AE, Szulc J, Borkhataria R, Geluk M, Haas J, Hornung J, Jordan P, Kempf O, and Michalík J. 2008. Triassic. The geology of central Europe 2:749-821. 10.1144/CEV2P.1

Fernández Blanco MV, Ezcurra MD, and Bona P. 2020. New embryological and palaeontological evidence sheds light on the evolution of the archosauromorph ankle. Scientific reports 10:1-8. 10.1038/s41598-020-62033-8

Fichter J. 1995. Katalog des publizierten Materials aus der palaontologischen Sammlung des Naturkundemuseums der Stadt Kassel. Philippia 7:129-157.

Flynn JJ, Nesbitt SJ, Michael Parrish J, Ranivoharimanana L, and Wyss AR. 2010. A new species of Azendohsaurus (Diapsida: Archosauromorpha) from the Triassic Isalo Group of southwestern Madagascar: cranium and mandible. Palaeontology 53:669-688. 10.1111/j.14754983.2010.00954.x

Foth C, Ezcurra MD, Sookias RB, Brusatte SL, and Butler RJ. 2016. Unappreciated diversification of stem archosaurs during the Middle Triassic predated the dominance of dinosaurs. BMC evolutionary biology 16:188. 10.1186/s12862-016-0761-6

Ford DP, and Benson RB. 2018. A redescription of Orovenator mayorum (Sauropsida, Diapsida) using high-resolution $\mu \mathrm{CT}$, and the consequences for early amniote phylogeny. Papers in Palaeontology 5:197-239. 10.1002/spp2.1236

Ford DP, and Benson RB. 2020. The phylogeny of early amniotes and the affinities of Parareptilia and Varanopidae. Nature ecology \& evolution 4:57-65. 10.1038/s41559-019-1047-3

Formoso KK, Nesbitt SJ, Pritchard AC, Stocker MR, and Parker WG. 2019. A long-necked tanystropheid from the Middle Triassic Moenkopi Formation (Anisian) provides insights into the ecology and biogeography of tanystropheids. Palaeontologia Electronica 22:1-15. 10.26879/988

Fraser N. 1982. A new rhynchocephalian from the British Upper Trias. Palaeontology 25:709-725.

Fraser N, and Walkden G. 1984. The postcranial skeleton of the Upper Triassic sphenodontid Planocephalosaurus robinsonae. Palaeontology 27:575-595.

Fraser NC, and Furrer H. 2013. A new species of Macrocnemus from the Middle Triassic of the eastern Swiss Alps. Swiss Journal of Geosciences 106:199-206. 10.1007/s00015-013-0137-5

Fraser NC, Grimaldi DA, Olsen PE, and Axsmith B. 1996. A Triassic Lagerstätte from eastern North America. Nature 380:615-619. 10.1038/380615a0

Fraser NC, and Rieppel O. 2006. A new protorosaur (Diapsida) from the Upper Buntsandstein of the Black Forest, Germany. Journal of Vertebrate Paleontology 26:866-871. 10.1671/02724634(2006)26[866:anpdft]2.0.co;2

Fraser NC, Rieppel O, and Li C. 2013. A long-snouted protorosaur from the Middle Triassic of southern China. Journal of Vertebrate Paleontology 33:1120-1126. 10.1080/02724634.2013.764310

Peer) reviewing PDF | (2020:08:51657:1:1:NEW 28 Feb 2021) 
4522

4523

4524

4525

4526

4527

4528

4529

4530

4531

4532

4533

4534

4535

4536

4537

4538

4539

4540

4541

4542

4543

4544

4545

4546

4547

4548

4549

4550

4551

4552

4553

4554

4555

4556

4557

4558

4559

4560

4561

4562

4563

4564

4565

4566

4567

4568
Fröbisch J, Schoch RR, Müller J, Schindler T, and Schweiss D. 2011. A new basal sphenacodontid synapsid from the Late Carboniferous of the Saar-Nahe Basin, Germany. Acta Palaeontologica Polonica 56:113-120. 10.4202/app.2010.0039

Gans C, Darevski I, and Tatarinov LP. 1987. Sharovipteryx, a reptilian glider? Palaeobiology 13:415-426.

Gardner NM, Holiday CM, and O'Keefe FR. 2010. The Braincase of Youngina capensis (Reptilia, Dipsida): New Insights from High-Resolution CT Scanning of the Holotype. Palaeontologia Electronica 13: 1-16.

Garyainov V, and Rykov S. 1973. "Southeastern slope of the Voronezh anteclise, Obshchii Syrt, and Orenburg Fore-Urals". In: Kiparisova L, Radchenko G, and Gorskii V, eds. "Stratigraphy of the USSR: Triassic System". Moscow, 89-111 (in Russian).

Gauffre F-X. 1993. The prosauropod dinosaur Azendohsaurus laaroussii the upper Triassic of Morocco. Palaeontology 36:897-908.

Gauthier JA. 1984. A cladistic analysis of the higher categories of the Diapsida. PhD thesis, University of California, Berkeley:564 p.

Gauthier JA. 1994. The diversification of the amniotes. In Spencer RS ed Major features of vertebrate evolution Knoxville: The University of Tennessee:129-159.

Gauthier J, Estes R, and De Queiroz K. 1988a. A phylogenetic analysis of Lepidosauromorpha. In: Estes R, and Pregill G, eds. Phylogenetic relationships of the lizard families, essays commemoration Charles L Camp. Stanford, California: Stanford University Press, 15-98.

Gauthier JA, Kluge AG, and Rowe T. 1988b. Amniote phylogeny and the importance of fossils. Cladistics 4:105-209. 10.1111/j.1096-0031.1988.tb00514.x

Goloboff PA, and Catalano SA. 2016. TNT version 1.5, including a full implementation of phylogenetic morphometrics. Cladistics 32:221-238. 10.1111/cla.12160

Goodrich E. 1942. The hind foot of Youngina and fifth metatarsal in Reptilia. Journal of Anatomy 76:308.

Gottmann-Quesada A, and Sander PM. 2009. A redescription of the early archosauromorph Protorosaurus speneri Meyer, 1832, and its phylogenetic relationships. Palaeontographica Abteilung A:123-220. $10.1127 /$ pala/287/2009/123

Gow CE. 1975. The morphology and relationships of Youngina capensis Broom and Prolacerta broomi Parrington. Palaeontologica Africana 18:89-131.

Gower DJ. 1994. Morphology and relationships of the earliest archosaurs. Unpublished PhD thesis, University of Bristol, Bristol.

Gower DJ. 2003. Osteology of the early archosaurian reptile Erythrosuchus africanus, Broom. Annals of the South African Museum 110:1-88.

Gower DJ. 1996. The tarsus of erythrosuchid archosaurs, and implications for early diapsid phylogeny. Zoological Journal of the Linnean Society 116:347-375. 10.1111/j.1096-3642.1996.tb00128.x

Gower DJ. 1997. The braincase of the early archosaurian reptile Erythrosuchus africanus. Journal of Zoology 242:557-576. 10.1111/j.1469-7998.1997.tb03855.x

Gower DJ, and Sennikov AG. 1996. Morphology and phylogenetic informativeness of early archosaur braincases. Palaeontology 39:883-906.

Gower DJ, and Sennikov AG. 1997. Sarmatosuchus and the early history of the Archosauria. Journal of Vertebrate Paleontology 17:60-73. 10.1080/02724634.1997.10010954

Gower DJ, and Sennikov AG. 2000. Early archosaurs from Russia. In: Benton MJ, Kurochkin EN, Shishkin MA, and Unwin DM, eds. The age of dinosaurs in Russia and Mongolia. Cambridge: Cambridge University Press, 140-159.

Grand A, Corvez A, Duque Velez LM, and Laurin M. 2013. Phylogenetic inference using discrete characters: performance of ordered and unordered parsimony and of three-item statements. Biological Journal of the Linnean Society 110:914-930. 10.1111/bij.12159

Peer) reviewing PDF | (2020:08:51657:1:1:NEW 28 Feb 2021) 
4569

4570

4571

4572

4573

4574

4575

4576

4577

4578

4579

4580

4581

4582

4583

4584

4585

4586

4587

4588

4589

4590

4591

4592

4593

4594

4595

4596

4597

4598

4599

4600

4601

4602

4603

4604

4605

4606

4607

4608

4609

4610

4611

4612

4613

Gregory J. 1945. Osteology and relationships of Trilophosaurus. University of Texas Publication 4401:273359.

Groenewald GH, and Kitching JW. 1995. Biostratigraphy of the Lystrosaurus assemblage zone. In Biostratigraphy of the Beaufort Group (Karoo Supergroup), by Rubidge BS, eds Pretoria: Council for Geosciences 1.

Hammer $\varnothing$, Harper DA, and Ryan PD. 2001. PAST: Paleontological statistics software package for education and data analysis. Palaeontologia Electronica 4:1-9.

Hancox P. 2000. The continental Triassic of South Africa. Zentralblatt für Geologie und Paläontologie Teil I 1998:1285-1324.

Haubold HA, and Schaumberg G. 1985. Die Fossilien des Kupferschiefers: Pflanzen-und Tierwelt zu Beginn des Zechsteins; eine Erzlagerstätte und ihre Paläontologie. Wittenberg, Germany: Ziemsen Verlag.

Haughton S. 1922. On the reptilian genera Euparkeria Broom, and Mesosuchus Watson. Transactions of the Royal Society of South Africa 10:81-88. 10.1080/00359192209519270Haughton S. 1924a. On a skull and partial skeleton of Mesosuchus browni Watson. Transactions of the Royal Society of South Africa 12:17-26. 10.1080/00359192409519295

Heckert AB. 2004. Late Triassic microvertebrates from the lower Chinle Group (Otischalkian-Adamanian: Carnian), southwestern USA. New Mexico Museum of Natural History and Science 27:1-170.

Hoffman A. 1965. On the discovery of a new thecodont from the Middle Beaufort Beds. Navorsinge van die Nasionale Museum: Researches of the National Museum 2:33-39.

Hone DW, and Benton MJ. 2007. An evaluation of the phylogenetic relationships of the pterosaurs among archosauromorph reptiles. Journal of Systematic Palaeontology 5:465-469. $10.1017 /$ S1477201907002064

Hone DW, and Benton MJ. 2008. A new genus of rhynchosaur from the Middle Triassic of south-west England. Palaeontology 51:95-115. 10.1111/j.1475-4983.2007.00739.x

Hsiou AS, De França MAG, and Ferigolo J. 2015. New data on the Clevosaurus (Sphenodontia: Clevosauridae) from the Upper Triassic of southern Brazil. PLoS ONE 10:1-21. 10.1371/journal.pone.0137523

Hsiou AS, Nydam RL, Simões TR, Pretto FA, Onary S, Martinelli AG, Liparini A, de Vivar Martínez PRR, Soares $\mathrm{MB}$, and Schultz CL. 2019. A new clevosaurid from the Triassic (Carnian) of Brazil and the rise of sphenodontians in Gondwana. Scientific Reports 9:1-12. 10.1038/s41598-019-48297-9

Huene FRv. 1902. Übersicht über die Reptilien der Trias. Geologische und Paläontologische Abhandlungen (Neue Serie) 6:1-84.

Huene FRv. 1905. Über die Trias-Dinosaurier Europas. Zeitschrift der Deutschen Geologischen Gesellschaft 57:345-349.

Huene FRv. 1908. Die Dinosaurier der europäischen Triasformation mit Berücksichtigung der aussereuropäischen Vorkommnisse. Geologische und Paläontologische Abhandlungen Supplement 1:1-419.

Huene FRv. 1911. Über Erythrosuchus, Vertreter der neuen Reptil-Ordnung Pelycosimia. Geologische und Paläontologische Abhandlungen 10:1-60.

Huene FRv. 1931. Über Tanystropheus und verwandte Formen. Neues Jahrbuch für Geologie und Paläontologie, Beilagen-Band 67:65-86.

Huene FRv. 1940. Eine Reptilfauna aus der ältesten Trias Nordrußlands. Neues Jahrbuch für Mineralogie, Geologie und Paläontologie, Beilagen-Band, Abteilung B 84:1-23.

Huene FRv. 1946. Die grossen Stämme der Tetrapoden in den geologischen Zeiten. Biologisches Zentralblatt 65:268-275.

Peer) reviewing PDF | (2020:08:51657:1:1:NEW 28 Feb 2021) 
4614 Huene FRv. 1944. Über die systematische Stellung von Trachelosaurus aus dem Buntsandstein von

4615

4616

4617

4618

4619

4620

4621

4622

4623

4624

4625

4626

4627

4628

4629

4630

4631

4632

4633

4634

4635

4636

4637

4638

4639

4640

4641

4642

4643

4644

4645

4646

4647

4648

4649

4650

4651

4652

4653

4654

4655

4656

4657

4658

4659
Bernburg. Neues Jahrbuch für Mineralogie, Geologie und Paläontologie, Monatshefte, Abteilung B 6:170-174.

Huene FRv. 1948. Notes on Gwyneddosaurus. American Journal of Science 246:208-213. 10.2475/ajs.246.4.208

Huene FRv. 1954. Ein neuer Protorosauride. Neues Jahrbuch für Geologie und Paläontologie, Monatshefte 5:228-230.

Hutchinson M, Skinner A, and Lee M. 2012. Tikiguania and the antiquity of squamate reptiles (lizards and snakes). Biology Letters 8:665-669. 10.1098/rsbl.2011.1216

Huxley TH. 1871. A manual of the anatomy of vertebrated animals. London: J. \& A. Churchill.

Irmis RB, Mundil R, Martz JW, and Parker WG. 2011. High-resolution U-Pb ages from the Upper Triassic Chinle Formation (New Mexico, USA) support a diachronous rise of dinosaurs. Earth and Planetary Science Letters 309:258-267. 10.1016/j.epsl.2011.07.015

Ivakhnenko MF, and Kurochkin EN. 2008. Fossil Reptiles and Birds Part 1. Moscow: GEOS.

Jalil N-E. 1990. Sur deux crânes de petits Sauria (Amniota, Diapsida) du Trias moyen d'Algérie. Comptes rendus de l'Académie des sciences Série 2, Mécanique, Physique, Chimie, Sciences de l'univers, Sciences de la Terre 311:731-736.

Jalil N-E. 1997. A new prolacertiform diapsid from the Triassic of North Africa and the interrelationships of the Prolacertiformes. Journal of Vertebrate Paleontology 17:506-525. 10.1080/02724634.1997.10010998

Jalil N-E. 1999. Continental Permian and Triassic vertebrate localities from Algeria and Morocco and their stratigraphical correlations. Journal of African Earth Sciences 29:219-226. 10.1016/S08995362(99)00091-3

Jalil N, and Knoll F. 2002. Is Azendohsaurus laaroussii (Carnian, Morocco) a dinosaur? Journal of Vertebrate Paleontology 22:70A.

Jaquier VP, Fraser NC, Furrer H, and Scheyer TM. 2017. Osteology of a new specimen of Macrocnemus aff. M. fuyuanensis (Archosauromorpha, Protorosauria) from the Middle Triassic of Europe: Potential implications for species recognition and paleogeography of tanystropheid protorosaurs. Frontiers in Earth Science 5:1-28. 10.3389/feart.2017.00091

Jaquier VP, and Scheyer TM. 2017. Bone histology of the Middle Triassic long-necked reptiles Tanystropheus and Macrocnemus (Archosauromorpha, Protorosauria). Journal of Vertebrate Paleontology 37:e1296456. 10.1080/02724634.2017.1296456

Jiang D-Y, Rieppel O, Fraser NC, Motani R, Hao W-C, Tintori A, Sun Y-L, and Sun Z-Y. 2011. New information on the protorosaurian reptile Macrocnemus fuyuanensis Li et al., 2007, from the Middle/Upper Triassic of Yunnan, China. Journal of Vertebrate Paleontology 31:1230-1237. 10.1080/02724634.2011.610853

Jiang D, Motani R, Hao W, Rieppel O, Sun Y, Tintori A, Sun Z, and Schmitz L. 2009. Biodiversity and sequence of the Middle Triassic Panxian marine reptile fauna, Guizhou Province, China. Acta Geologica Sinica-English Edition 83:451-459. 10.1111/j.1755-6724.2009.00047.x

Kearney M, and Rieppel O. 2006. Rejecting "the given" in systematics. Cladistics 22:369-377. 10.1111/j.1096-0031.2006.00110.x

Kligman BT, Marsh AD, Nesbitt SJ, Parker WG, and Stocker MR. 2020. New trilophosaurid species demonstrates a decline in allokotosaur diversity across the Adamanian-Revueltian boundary in the Late Triassic of western North America. Palaeodiversity 13:25-37. 10.18476/pale.v13.a3

Kuhn-Schnyder E. 1947. Der Schädel von Tanystropheus. Eclogae Geologicae Helvetiae 40:390.

Kuhn-Schnyder E. 1954. Über die Herkunft der Eidechsen. Endeavour 13:215-221.

Peer) reviewing PDF | (2020:08:51657:1:1:NEW 28 Feb 2021) 
4660 Kuhn-Schnyder E. 1959. Hand und Fuss von Tanystropheus longobardicus (Bassani). Eclogae Geologicae Helvetiae 52:921-941.

Kuhn-Schnyder E. 1962. Ein weiterer Schädel von Macrocnemus bassanii Nopcsa aus der anisischen Stufe der Trias des Monte San Giorgio (Kt. Tessin, Schweiz). Paläontologische Zeitschrift 36:110-133. 10.1007/BF02987896

Kuhn-Schnyder E. 1963. Wege der Reptiliensystematik. Paläontologische Zeitschrift 37:61-87. 10.1007/BF02989601

Kuhn-Schnyder E. 1967. Das Problem der Euryapsida. Éditions du Centre national de la recherche scientifique 163:335-348.

Kuhn-Schnyder E. 1974. Die Triasfauna der Tessiner Kalkalpen. Vierteljahresschrift der naturforschenden Gesellschaft in Zürich, Neuljahrsblatt 118:1-119.

Kuhn O. 1971. Die Saurier der Deutschen Trias. Altötting: Geiselberger.

LaBarbera MC, and Rieppel O. 2005. Suction feeding in a Triassic protorosaur? Response. Science 308:1113. 10.1126/science.308.5725.1112c

Lane H. 1945. New mid-Pennsylvanian reptiles from Kansas. Transactions of the Kansas Academy of Science 47:381-390. 10.2307/3625454

Langer MC, Ribeiro AM, Schultz CL, and Ferigolo J. 2007. The continental tetrapod-bearing Triassic of south Brazil. New Mexico Museum of Natural History and Science Bulletin 41:201-218.

Langer MC, and Schultz CL. 2000. A new species of the Late Triassic rhynchosaur Hyperodapedon from the Santa Maria Formation of south Brazil. Palaeontology 43:633-652. 10.1111/1475-4983.00143

Laurin M. 1991. The osteology of a Lower Permian eosuchian from Texas and a review of diapsid phylogeny. Zoological Journal of the Linnean Society 101:59-95. 10.1111/j.10963642.1991.tb00886.x

Legler B, and Schneider JW. 2008. Marine ingressions into the Middle/Late Permian saline lake of the Southern Permian Basin (Rotliegend, Northern Germany) possibly linked to sea-level highstands in the Arctic rift system. Palaeogeography, Palaeoclimatology, Palaeoecology 267:102-114. 10.1016/j.palaeo.2008.06.009

Lehman J-P. 1971. Nouveaux vertébrés fossiles du Trias de la Série de Zarzaitine. Annales de Paléontologie (Vertébrés) 57:71-93.

Lessner EJ, Parker WG, Marsh AD, Nesbitt SJ, Irmis RB, and Mueller BD. 2018. New insights into Late Triassic dinosauromorph-bearing assemblages from Texas using apomorphy-based identifications. PaleoBios 35:1-41.

Li C. 2003. First record of protorosaurid reptile (Order Protorosauria) from the Middle Triassic of China. Acta Geologica Sinica 77:419-423. 10.1111/j.1755-6724.2003.tb00122.x

Li C. 2007. A juvenile Tanystropheus sp. (Protorosauria, Tanystropheidae) from the Middle Triassic of Guizhou, China. Vertebrata PalAsiatica 45: 37-42.

Li C, Fraser NC, Rieppel O, and Wu X-C. 2018. A Triassic stem turtle with an edentulous beak. Nature 560:476-479. 10.1038/s41586-018-0419-1

Li C, Fraser NC, Rieppel O, Zhao L-J, and Wang L-T. 2017a. A new diapsid from the Middle Triassic of southern China. Journal of Paleontology:1-7. 10.1017/jpa.2017.12

Li C, Rieppel O, and Fraser NC. 2017b. Viviparity in a Triassic marine archosauromorph reptile. Vertebrata PalAsiatica 55:210-217.

Li C, Rieppel O, and LaBarbera MC. 2004. A Triassic aquatic protorosaur with an extremely long neck. Science 305:1931. 10.1126/science.1100498

Li C, Zhao L-J, and Wang L-T. 2007. A new species of Macrocnemus (Reptilia: Protorosauria) from the Middle Triassic of southwestern China and its palaeogeographical implication. Science in China Series D: Earth Sciences 50:1601-1605. 10.1007/s11430-007-0118-5 
4707

4708

4709

4710

4711

4712

4713

4714

4715

4716

4717

4718

4719

4720

4721

4722

4723

4724

4725

4726

4727

4728

4729

4730

4731

4732

4733

4734

4735

4736

4737

4738

4739

4740

4741

4742

4743

4744

4745

4746

4747

4748

4749

4750

4751

Liu J, Organ CL, Benton MJ, Brandley MC, and Aitchison JC. 2017. Live birth in an archosauromorph reptile. Nature communications 8:14445. 10.1038/ncomms14445

Liutkus-Pierce CM, Fraser NC, and Heckert AB. 2014. Stratigraphy, sedimentology, and paleontology of the Upper Triassic Solite Quarry, North Carolina and Virginia. Elevating Geoscience in the Southeastern United States: New Ideas about Old Terranes-Field Guides for the 2014 GSA Southeastern Section Meeting, Blacksburg, Virginia: Boulder, CO, Geological Society of America. p 255-269.

Lucas SG. 2010. The Triassic timescale based on nonmarine tetrapod biostratigraphy and biochronology. Geological Society, London, Special Publications 334:447-500. 10.1144/SP334.15

MacDougall MJ, Tabor NJ, Woodhead J, Daoust AR, and Reisz RR. 2017. The unique preservational environment of the Early Permian (Cisuralian) fossiliferous cave deposits of the Richards Spur locality, Oklahoma. Palaeogeography, Palaeoclimatology, Palaeoecology 475:1-11. 10.1016/j.palaeo.2017.02.019

Maidment SC, Sennikov AG, Ezcurra MD, Dunne EM, Gower DJ, Hedrick BP, Meade LE, Raven TJ, Paschchenko DI, and Butler RJ. 2020. The postcranial skeleton of the erythrosuchid archosauriform Garjainia prima from the Early Triassic of European Russia. Royal Society Open Science 7:201089. 10.1098/rsos.201089

Melville RV. 1981. Opinion 1186 Tanystropheus H. Von Meyer, [1852] (Reptilia) conserved. Bulletin of Zoological Nomenclature 38:188-190.

Menning M, and Hendrich A. 2016. Deutsche Stratigraphische Kommission: Stratigraphische Tabelle von Deutschland 2016.

Merck J. 1997. A phylogenetic analysis of the euryapsid reptiles. PhD dissertation, University of Texas at Austin, Austin, Texas:785 p.

Meyer Hv. 1830. Protorosaurus. Isis von Oken:517-519.

Meyer Hv. 1832. Palaeologica zur Geschichte der Erde und ihrer Geschöpfe. S Schmerber.

Meyer Hv. 1855. Die Saurier des Muschelkalkes mit Rücksicht auf die Saurier aus buntem Sandstein und Keuper. In: Keller H, ed. Zur Fauna der Vorwelt, zweite Abtheilung. Frankfurt a.m.: Heinrich Keller.

Meyer Hv. 1856. Zur Fauna der Vorwelt-Saurier aus dem Kupferschiefer der Zechstein-Formation. Keller:24 p.

Miedema F, Spiekman SNF, Fernandez V, Reumer JWF, and Scheyer TM. 2020. Cranial morphology of the tanystropheid Macrocnemus bassanii unveiled using synchrotron microtomography. Scientific reports 10:12412. 10.1038/s41598-020-68912-4

Modesto SP, and Sues H-D. 2004. The skull of the Early Triassic archosauromorph reptile Prolacerta broomi and its phylogenetic significance. Zoological Journal of the Linnean Society 140:335-351. 10.1111/j.1096-3642.2003.00102.x

Montefeltro FC, Langer MC, and Schultz CL. 2010. Cranial anatomy of a new genus of hyperodapedontine rhynchosaur (Diapsida, Archosauromorpha) from the Upper Triassic of southern Brazil. Earth and Environmental Science Transactions of the Royal Society of Edinburgh 101:27-52. 10.1017/S1755691010009060

Mueller B, and Parker W. 2006. A new species of Trilophosaurus (Diapsida: Archosauromorpha) from the Sonsela Member (Chinle Formation) of Petrified Forest National Park, Arizona. Museum of Northern Arizona, Bulletin 62:119-125.

Müller J. 2004. The relationships among diapsid reptiles and the influence of taxon selection. In Recent advances in the origin and early radiation of vertebrates, by Arratia $G$, Wilson $M V H$, and Cloutier $R$, eds Verlag Dr Friedrich Pfeil, München:379-408.

Peer) reviewing PDF | (2020:08:51657:1:1:NEW 28 Feb 2021) 
4752

4753

4754

4755

4756

4757

4758

4759

4760

4761

4762

4763

4764

4765

4766

4767

4768

4769

4770

4771

4772

4773

4774

4775

4776

4777

4778

4779

4780

4781

4782

4783

4784

4785

4786

4787

4788

4789

4790

4791

4792

4793

4794

4795

4796

4797

4798
Muscio G. 1996. Preliminary note on a specimen of Prolacertiformes (Reptilia) from the Norian (Late Triassic) of Preone (Udine, North-Eastern Italy). Gortania - Atti del Museo Friulano di Storia Naturale 18:33-40.

Nesbitt SJ. 2011. The early evolution of archosaurs: relationships and the origin of major clades. Bulletin of the American Museum of Natural History 352:1-292 10.1206/352.1

Nesbitt SJ, Butler RJ, Ezcurra MD, Barrett PM, Stocker MR, Angielczyk KD, Smith RM, Sidor CA, Niedźwiedzki G, and Sennikov AG. 2017a. The earliest bird-line archosaurs and the assembly of the dinosaur body plan. Nature 544:484-487. 10.1038/nature22037

Nesbitt SJ, Flynn JJ, Pritchard AC, Parrish JM, Ranivoharimanana L, and Wyss AR. 2015. Postcranial anatomy and relationships of Azendohsaurus madagaskarensis. Bulletin of the American Museum of Natural History 398:1-126. 10.1206/amnb-899-00-1-126.1

Nesbitt SJ, Stocker MR, Ezcurra MD, Fraser NC, Heckert AB, Marsh A, Parker W, Mueller B, and Pritchard AC. 2017b. The 'strange reptiles' of the Triassic: the morphology, ecology, and taxonomic diversity of the clade Allokotosauria illuminated by the discovery of an early diverging member. SVP Program and Abstracts 2017:168.

Nopcsa FB. 1923. Neubeschreibung des Trias-Pterosauriers Tribelesodon. Paläontologische Zeitschrift 5:161-181. 10.1007/BF03160365

Nopcsa FB. 1930. Notizen über Macrochemus Bassanii nov. gen. et spec. Centralblatt für Mineralogie, Geologie und Paläontologie B 7:252-255.

Nosotti S. 2007. Tanystropheus longobardicus (Reptilia, Protorosauria): Re-interpretations of the anatomy based on new specimens from the Middle Triassic of Besano (Lombardy, northern Italy). Memorie della Società Italiana di Scienze Naturali e del Museo Civico di Storia Naturale di Milano 35:1-88.

Ochev VG. 1979. New Lower Triassic archosaurs from the eastern part of the European USSR. Palaeontological Journal 1:104-109.

Ochev VG. 1986. Concerning the Middle Triassic reptiles from the southern Cis-Ural region. Ezhegodnik Vsesoyuznogo Paleontologicheskogo Obshchestva 29:171-179.

Olsen PE. 1979. A new aquatic eosuchian from the Newark Supergroup (Late Triassic-Early Jurassic) of North Carolina and Virginia. Postilla 176:1-14.

Olson EC, and Broom R. 1937. New genera and species of tetrapods from the Karroo beds of South Africa. Journal of Paleontology:613-619.

Ortlam D. 1966. Fossile Böden als Leithorizonte für die Gliederung des Höheren Buntsandsteins im nördlichen Schwarzwald und südlichen Odenwald. Geologisches Jahrbuch 84:485-590.

Osborn HF. 1903. The reptilian subclasses Diapsida and Synapsida and the early history of the Diaptosauria. Memoirs of the American Museum of Natural History 1:449-519.

Owen R. 1842. Description of parts of the skeleton and teeth of five species of the genus Labyrinthodon (Lab. leptognathus, Lab. pachygnathus, and Lab. ventricosus, from the Coton-end and Cubbington Quarries of the Lower Warwick Sandstone; Lab. Jaegeri, from Guy's Cliff, Warwick; and Lab. scutulatus, from Leamington); with remarks on the probable identity of the Cheirotherium with this genus of extinct batrachians. Transactions of the Geological Society, London 2:515-543. 10.1144/transgslb.6.2.515

Padian K. 1997. Pterosauromorpha. In Encyclopedia of dinosaurs, by Currie, PJ, and Padian, K, eds San Diego, Academic Press:617-618.

Padian K, and Chiappe LM. 1998. The origin and early evolution of birds. Biological Reviews 73:1-42.

Parks P. 1969. Cranial anatomy and mastication of the Triassic reptile Trilophosaurus MSc. University of Texas at Austin.

Parrington FR. 1935. On Prolacerta broomi, gen. et sp. n. and the origin of lizards. Annals and Magazine of Natural History Series 10 16:197-205. 10.1080/00222933508655037

Peer) reviewing PDF | (2020:08:51657:1:1:NEW 28 Feb 2021) 
4799

4800

4801

4802

4803

4804

4805

4806

4807

4808

4809

4810

4811

4812

4813

4814

4815

4816

4817

4818

4819

4820

4821

4822

4823

4824

4825

4826

4827

4828

4829

4830

4831

4832

4833

4834

4835

4836

4837

4838

4839

4840

4841

4842

4843

4844

4845
Parrington FR. 1956. A problematic reptile from the Upper Permian. The Annals and Magazine of Natural History; Zoology, Botany, and Geology 12:333-336. 10.1080/00222935608655819

Parrish JM. 1992. Phylogeny of the Erythrosuchidae (Reptilia: Archosauriformes). Journal of Vertebrate Paleontology 12:93-110. 10.1080/02724634.1992.10011434

Peabody FE. 1952. Petrolacosaurus kansensis Lane, a Pennsylvanian reptile from Kansas. University of Kansas Paleontological Contributions - Vertebrata:1-41.

Peecook BR, Smith RM, and Sidor CA. 2019. A novel archosauromorph from Antarctica and an updated review of a high-latitude vertebrate assemblage in the wake of the end-Permian mass extinction. Journal of Vertebrate Paleontology 38: e1536664. 10.1080/02724634.2018.1536664

Peters D. 2000. A reexamination of four Prolacertiformes with implications for pterosaur phylogenesis. Rivista Italiana di Paleontologia e Stratigrafia 106:293-336. 10.13130/2039-4942/6148

Peters D. 2005. Suction feeding in a Triassic protorosaur? Science 308:1112. 10.1126/science.308.5725.1112c

Peyer B. 1930. Tanystropheus longobardicus Bass. sp. (Vorläufige Mitteilung). Centralblatt für Mineralogie, Geologie und Paläontologie B 8:336-337.

Peyer B. 1931. Die Triasfauna der Tessiner Kalkalpen II. Tanystropheus longobardicus Bass. sp. Abhandlungen der Schweizerischen Paläontologischen Gesellschaft 50:9-110.

Peyer B. 1937. Die Triasfauna der Tessiner Kalkalpen XII. Macrocnemus bassanii Nopcsa. Abhandlungen der Schweizerischen Paläontologischen Gesellschaft 54:1-87.

Pinheiro FL, França MA, Lacerda MB, Butler RJ, and Schultz CL. 2016. An exceptional fossil skull from South America and the origins of the archosauriform radiation. Scientific Reports 6:22817. $10.1038 /$ srep22817

Pinheiro FL, Simão-Oliveira DD, and Butler RJ. 2019. Osteology of the archosauromorph Teyujagua paradoxa and the early evolution of the archosauriform skull. Zoological Journal of the Linnean Society 189:378-417. 10.1093/zoolinnean/zlz093

Pinna G. 1980. Drepanosaurus unguicaudatus, nuovo genere e nuova specie di Lepidosauro del trias alpino (Reptilia). Atti della Società italiana di scienze naturali e del Museo civico di storia naturale di Milano 121:181-192.

Pinna G. 1984. Osteologia di Drepanosaurus unguicaudatus, Lepidosauro triassico del Sottordine Lacertilia. Atti della Società Italiana Scienze Naturali 124:7-28.

Pinna G. 1986. On Drepanosaurus unguicaudatus, an Upper Triassic Lepidosaurian from the Italian Alps. Journal of Paleontology 60:1127-1132.

Pinna G. 1993. The Norian Reptiles from Northern Italy. Paleontologia Lombarda Nuova Serie 2:115-124.

Pol D, and Escapa IH. 2009. Unstable taxa in cladistic analysis: identification and the assessment of relevant characters. Cladistics 25:515-527. 10.1111/j.1096-0031.2009.00258.x

Premru E. 1991. Beschreibung eines neuen Fundes von Macrocnemus bassanii Nopcsa (Reptilia, Squamata, Prolacertiformes) aus der Grenzbitumenzone (Anis/Ladin) des Monte San Giorgio (Besano, I). Diplomarbeit an der Philosophischen Fakultät II der Universität Zürich.

Pritchard AC, Gauthier JA, Hanson M, Bever GS, and Bhullar B-AS. 2018. A tiny Triassic saurian from Connecticut and the early evolution of the diapsid feeding apparatus. Nature communications 9:1213. 10.1038/s41467-018-03508-1

Pritchard AC, and Nesbitt SJ. 2017. A bird-like skull in a Triassic diapsid reptile increases heterogeneity of the morphological and phylogenetic radiation of Diapsida. Royal Society Open Science 4: 170499. 10.1098/rsos.170499

Pritchard AC, and Sues H-D. 2019. Postcranial remains of Teraterpeton hrynewichorum (Reptilia: Archosauromorpha) and the mosaic evolution of the saurian postcranial skeleton. Journal of Systematic Palaeontology 17:1745-1765. 10.1080/14772019.2018.1551249

Peer) reviewing PDF | (2020:08:51657:1:1:NEW 28 Feb 2021) 
4846

4847

4848

4849

4850

4851

4852

4853

4854

4855

4856

4857

4858

4859

4860

4861

4862

4863

4864

4865

4866

4867

4868

4869

4870

4871

4872

4873

4874

4875

4876

4877

4878

4879

4880

4881

4882

4883

4884

4885

4886

4887

4888

4889

4890

4891

4892

4893

Pritchard AC, Turner AH, Irmis RB, Nesbitt SJ, and Smith ND. 2016. Extreme modification of the tetrapod forelimb in a Triassic diapsid reptile. Current Biology 26:1-8. 10.1016/j.cub.2016.07.084

Pritchard AC, Turner AH, Nesbitt SJ, Irmis RB, and Smith ND. 2015. Late Triassic tanystropheids (Reptilia, Archosauromorpha) from Northern New Mexico (Petrified Forest Member, Chinle Formation) and the biogeography, functional morphology, and evolution of Tanystropheidae. Journal of Vertebrate Paleontology 35:e911186. 10.1080/02724634.2014.911186

Rauhut OWM, Heyng AM, López-Arbarello A, and Hecker A. 2012. A New Rhynchocephalian from the Late Jurassic of Germany with a Dentition That Is Unique amongst Tetrapods. PLOS ONE 7: e46839. 10.1371/journal.pone.0046839

Reisz RR. 1977. Petrolacosaurus, the oldest known diapsid reptile. Science 196:1091-1093. 10.1126/science.196.4294.1091

Reisz RR. 1981. A diapsid reptile from the Pennsylvanian of Kansas. Special Publication of the Natural History Museum, University of Kansas 7.

Reisz RR, Berman DS, and Scott DM. 1984. The anatomy and relationships of the Lower Permian reptile Araeoscelis. Journal of Vertebrate Paleontology 4:57-67. 10.1080/02724634.1984.10011986

Reisz RR, Heaton MJ, and Pynn BR. 1982. Vertebrate fauna of late Pennsylvanian rock lake shale near Garnett, Kansas: Pelycosauria. Journal of Paleontology 56:741-750.

Reisz RR, Laurin M, and Marjanović D. 2010. Apsisaurus witteri from the Lower Permian of Texas: yet another small varanopsid synapsid, not a diapsid. Journal of Vertebrate Paleontology 30:16281631. 10.1080/02724634.2010.501441

Reisz RR, Modesto SP, and Scott DM. 2000. Acanthotoposaurus bremneri and the origin of the Triassic archosauromorph reptile fauna of South Africa. South African Journal of Science 96:443-445.

Reisz RR, Modesto SP, and Scott DM. 2011. A new Early Permian reptile and its significance in early diapsid evolution. Proceedings of the Royal Society B: Biological Sciences 278:3731-3737. 10.1098/rspb.2011.0439

Renesto S. 1994a. Megalancosaurus, a possibly arboreal archosauromorph (Reptilia) from the Upper Triassic of northern Italy. Journal of Vertebrate Paleontology 14:38-52. 10.1080/02724634.1994.10011537

Renesto S. 1994b. A new prolacertiform reptile from the Late Triassic of Northern Italy. Rivista Italiana di Paleontologia e Stratigrafia 100:285-306.

Renesto S. 1994c. The shoulder girdle and anterior limb of Drepanosaurus unguicaudatus (Reptilia, Neodiapsida) from the upper Triassic (Norian) of Northern Italy. Zoological Journal of the Linnean Society 111:247-264. 10.1111/j.1096-3642.1994.tb01485.x

Renesto S. 2000. Bird-like head on a chameleon body: new specimens of the enigmatic diapsid reptile Megalancosaurus from the Late Triassic of Northern Italy. Rivista Italiana di Paleontologia $e$ Stratigrafia 106:157-180. 10.13130/2039-4942/5396

Renesto S. 2005. A new specimen of Tanystropheus (Reptilia Protorosauria) from the Middle Triassic of Switzerland and the ecology of the genus. Rivista Italiana di Paleontologia e Stratigrafia 111:377394. 10.13130/2039-4942/6327

Renesto S, and Avanzini M. 2002. Skin remains in a juvenile Macrocnemus bassanii Nopcsa (Reptilia, Prolacertiformes) from the Middle Triassic of northern Italy. Neues Jahrbuch für Geologie und Paläontologie Abhandlungen:31-48. 10.1127/njgpa/224/2002/31

Renesto S, and Binelli G. 2006. Vallesaurus cenensis Wild, 1991, a Drepanosaurid (Reptilia, Diapsida) from the Late Triassic of Northern Italy. Rivista Italiana di Paleontologia e Stratigrafia 112:77-94. 10.13130/2039-4942/5851

Renesto S, and Dalla Vecchia FM. 2000. The unusual dentition and feeding habits of the prolacertiform reptile Langobardisaurus (Late Triassic, Northern Italy). Journal of Vertebrate Paleontology 20:622-627. 10.1671/0272-4634(2000)020[0622:tudafh]2.0.co;2

Peer) reviewing PDF | (2020:08:51657:1:1:NEW 28 Feb 2021) 
4894

4895

4896

4897

4898

4899

4900

4901

4902

4903

4904

4905

4906

4907

4908

4909

4910

4911

4912

4913

4914

4915

4916

4917

4918

4919

4920

4921

4922

4923

4924

4925

4926

4927

4928

4929

4930

4931

4932

4933

4934

4935

4936

4937

4938

4939

4940

4941

Renesto S, and Dalla Vecchia FM. 2005. The skull of the holotype of Megalancosaurus preonensis (Diapsida, Drepanosauridae) from the Upper Triassic of Northern Italy. Rivista Italiana di Paleontologia e Stratigrafia 111:247-257.

Renesto S, and Dalla Vecchia FM. 2007. A revision of Langobardisaurus rossii Bizzarini and Muscio, 1995 from the Late Triassic of Friuli (Italy). Rivista Italiana di Paleontologia e Stratigrafia 113:191-201. 10.13130/2039-4942/5870

Renesto S, Dalla Vecchia FM, and Peters D. 2002. Morphological evidence for bipedalism in the Late Triassic prolacertiform reptile Langobardisaurus. Senckenbergiana lethaea 82:95-106. 10.1007/BF03043775

Renesto S, and Paganoni A. 1995. A new Drepanosaurus (Reptilia, Neodiapsida) from the Upper Triassic of Northern Italy. Neues Jahrbuch für Geologie und Paläontologie Abhandlungen 197:87-99. 10.1127/njgpa/197/1995/87

Renesto S, Spielmann JA, Lucas SG, and Spagnoli GT. 2010. The taxonomy and paleobiology of the Late Triassic (Carnian-Norian: Adamanian-Apachean) drepanosaurs (Diapsida: Archosauromorpha: Drepanosauromorpha): Bulletin 46. New Mexico Museum of Natural History and Science 46.

Rieppel O. 1989. The hind limb of Macrocnemus bassani (Nopcsa) (Reptilia, Diapsida): Development and functional anatomy. Journal of Vertebrate Paleontology 9:373-387. 10.1080/02724634.1989.10011771

Rieppel O. 1994. Osteology of Simosaurus and the interrelationships of stem-group Sauropterygia (Reptilia, Diapsida). Fieldiana, Geology, New Series 28:1-28.

Rieppel O. 2001. A new species of Tanystropheus (Reptilia: Protorosauria) from the Middle Triassic of Makhtesh Ramon, Israel. Neues Jahrbuch für Geologie und Paläontologie Abhandlungen 221:271287. 10.1127/njgpa/221/2001/271

Rieppel O. 2002. Feeding mechanics in Triassic stem-group sauropterygians: the anatomy of a successful invasion of Mesozoic seas. Zoological Journal of the Linnean Society 135:33-63. 10.1046/j.10963642.2002.00019.x

Rieppel O, Fraser NC, and Nosotti S. 2003. The monophyly of Protorosauria (Reptilia, Archosauromorpha): a preliminary analysis. Atti della Società italiana di scienze naturali e del Museo civico di storia naturale di Milano 144:359-382.

Rieppel O, and Gronowski RW. 1981. The loss of the lower temporal arcade in diapsid reptiles. Zoological Journal of the Linnean Society 72:203-217. 10.1111/j.1096-3642.1981.tb01570.x

Rieppel O, Jiang D-Y, Fraser NC, Hao W-C, Motani R, Sun Y-L, and Sun Z-Y. 2010. Tanystropheus cf. T. longobardicus from the early Late Triassic of Guizhou Province, southwestern China. Journal of Vertebrate Paleontology 30:1082-1089. 10.1080/02724634.2010.483548

Rieppel O, and Kearney M. 2007. The poverty of taxonomic characters. Biology \& Philosophy 22:95-113. 10.1007/s10539-006-9024-z

Rieppel O, Li C, and Fraser NC. 2008. The skeletal anatomy of the Triassic protorosaur Dinocephalosaurus orientalis, from the Middle Triassic of Guizhou Province, southern China. Journal of Vertebrate Paleontology 28:95-110. 10.1671/0272-4634(2008)28[95:tsaott]2.0.co;2

Rieppel O, Mazin J-M, and Tchernov E. 1999. Sauropterygia from the Middle Triassic of Makhtesh Ramon, Negev, Israel. Fieldiana, Geology, New Series 40:1-85.

Rigo M, Galli M, and Jadoul F. 2009. Late Triassic biostratigraphic constraints in the Imagna Valley (western Bergamasc Alps, Italy). Albertiana 37:39-42.

Romer AS. 1956. Osteology of the Reptiles. Chicago: University of Chicago Press:772 p.

Romer AS. 1966. Vertebrate paleontology. Chicago: University of Chicago Press 3rd edition:468 p.

Romer AS. 1968. Notes and Comments on Vertebrate Paleontology. The University of Chicago Press.

Rubidge BS. 2005. Re-uniting lost continents-fossil reptiles from the ancient Karoo and their wanderlust. South African Journal of Geology 108:135-172. 10.2113/108.1.135

Peer) reviewing PDF | (2020:08:51657:1:1:NEW 28 Feb 2021) 
4942

4943

4944

4945

4946

4947

4948

4949

4950

4951

4952

4953

4954

4955

4956

4957

4958

4959

4960

4961

4962

4963

4964

4965

4966

4967

4968

4969

4970

4971

4972

4973

4974

4975

4976

4977

4978

4979

4980

4981

4982

4983

4984

4985

4986

4987

Rubidge BS, Erwin DH, Ramezani J, Bowring SA, and de Klerk WJ. 2013. High-precision temporal calibration of Late Permian vertebrate biostratigraphy: U-Pb zircon constraints from the Karoo Supergroup, South Africa. Geology 41:363-366. 10.1130/G33622.1

Rykov S, and Ochev V. 1966. "On localities of Triassic vertebrates in Donskaya Luka." Questions of Geology of the Southern Ural Mountains and Volga Region (Saratov Gos Univ, Saratov, 1966) 1:58-62 (in Russian).

Saller F, Renesto S, and Dalla Vecchia FM. 2013. First record of Langobardisaurus (Diapsida, Protorosauria) from the Norian (Late Triassic) of Austria, and a revision of the genus. Neues Jahrbuch für Geologie und Paläontologie Abhandlungen 268:83-95. 10.1127/0077-7749/2013/0319

Sanz JL, and López-Martínez N. 1984. The prolacertid lepidosaurian Cosesaurus aviceps Ellenberger \& Villalta, a claimed «protoavian» from the Middle Triassic of Spain. Geobios 17:747-755. 10.1016/S0016-6995(84)80119-9

Scheyer TM, Spiekman SNF, Butler RJ, Ezcurra MD, Sues H-D, and Jones MEH. 2020a. Colobops, a juvenile rhynchocephalian reptile (Lepidosauromorpha), not a diminutive archosauromorph with an unusually strong bite. Royal Society Open Science 7:1-14. 10.1098/rsos.192179

Scheyer TM, Wang W, Li C, Miedema F, and Spiekman SNF. 2020b. Osteological re-description of Macrocnemus fuyuanensis (Archosauromorpha, Tanystropheidea) from the Middle Triassic of China. Vertebrata PalAsiatica 58:169-187. 10.19615/j.cnki.1000-3118.200525

Schmidt M. 1928. Die Lebewelt unserer Trias. Öhringen: Hohenlohe'sche Buchhandlung.

Schmidt M. 1938. Die Lebewelt unserer Trias (Nachtrag). Öhringen: Hohenlohe'sche Buchhandlung.

Schoch RR. 2019. Osteology of the temnospondyl Trematosaurus brauni Burmeister, 1849 from the Middle Buntsandstein of Bernburg, Germany. Palaeodiversity 12:41-63. 10.18476/pale.v12.a4

Sen K. 2003. Pamelaria dolichotrachela, a new prolacertid reptile from the Middle Triassic of India. Journal of Asian Earth Sciences 21:663-681. 10.1016/S1367-9120(02)00110-4

Sengupta S, Ezcurra MD, and Bandyopadhyay S. 2017. A new horned and long-necked herbivorous stemarchosaur from the Middle Triassic of India. Scientific Reports 7:8366. 10.1038/s41598-01708658-8

Sennikov A. 1995. "Early thecodonts of eastern Europe". Trudy Paleontologischeskogo Instituta RAN 263:1-141 (in Russian).

Sennikov AG. 1997. An enigmatic reptile from the Upper Permian of the Volga River Basin. Paleontological Journal 31:94-101.

Sennikov AG. 2005. A new specialized prolacertilian (Reptilia: Archosauromorpha) from the Lower Triassic of the Orenburg Region. Paleontological Journal 39:199-209.

Sennikov AG. 2011. New tanystropheids (Reptilia: Archosauromorpha) from the Triassic of Europe. Paleontological Journal 45:90-104. 10.1134/s0031030111010151

Sennikov A, and Golubev V. 2012. On the faunal verification of the Permo-Triassic boundary in continental deposits of eastern Europe: 1. Gorokhovets-Zhukov ravine. Paleontological Journal 46:313-323. $10.1134 / \mathrm{S} 003103011203015 \mathrm{X}$

Senter P. 2004. Phylogeny of Drepanosauridae (Reptilia: Diapsida). Journal of Systematic Palaeontology 2:257-268. 10.1017/S1477201904001427

Sereno PC. 1991. Basal archosaurs: phylogenetic relationships and functional implications. Journal of Vertebrate Paleontology 11:1-53. 10.1080/02724634.1991.10011426

Sharov AG. 1970. "Unusual reptile from the Lower Triassic of Fergana". Paleontologicheskii Zhurnal 1:127131 (in Russian).

Sharov AG. 1971. "New flying reptiles from the Mesozoic of Kazakhstan and Kirgizstan". Trudy Paleontologicheskogo Instituta AN SSSR 130:104-113 (in Russian).

Peer) reviewing PDF | (2020:08:51657:1:1:NEW 28 Feb 2021) 
4988

4989

4990

4991

4992

4993

4994

4995

4996

4997

4998

4999

5000

5001

5002

5003

5004

5005

5006

5007

5008

5009

5010

5011

5012

5013

5014

5015

5016

5017

5018

5019

5020

5021

5022

5023

5024

5025

5026

5027

5028

5029

5030

5031

5032

5033
Shishkin MA, and Sulej T. 2009. The Early Triassic temnospondyls of the Czatkowice 1 tetrapod assemblage. Palaeontologia Polonica 65:77.

Simões TR, Caldwell MW, Palci A, and Nydam RL. 2016. Giant taxon-character matrices: quality of character constructions remains critical regardless of size. Cladistics 33:198-219. 10.1111/cla.12163

Simões TR, Caldwell MW, Tałanda M, Bernardi M, Palci A, Vernygora O, Bernardini F, Mancini L, and Nydam RL. 2018. The origin of squamates revealed by a Middle Triassic lizard from the Italian Alps. Nature 557:706-709. 10.1038/s41586-018-0093-3

Skawiński T, Tałanda M, and Sachs S. 2015. Megacnemus - a forgotten reptile, presumably from the Triassic of Poland. EAVP Poster. 10.13140/RG.2.1.3950.7049

Skawiński T, Ziegler M, Czepiński Ł, Szermański M, Tałanda M, Surmik D, and Niedźwiedzki G. 2017. A reevaluation of the historical 'dinosaur' remains from the Middle-Upper Triassic of Poland. Historical Biology 29:442-472. 10.1080/08912963.2016.1188385

Smith R, Rubidge B, and Van der Walt M. 2012. Therapsid biodiversity patterns and paleoenvironments of the Karoo Basin, South Africa. In: Chinsamy-Turan A, ed. Forerunners of Mammals: Radiation, Histology, Biology Indiana University Press, Bloomington, Indiana. Bloomington, Indiana: Indiana University Press, 30-62.

Smith RMH, and Evans SE. 1996. New material of Youngina: evidence of juvenile aggregation in Permian diapsid reptiles. Palaeontology 39:289-303.

Sobral G, and Müller J. 2019. The braincase of Mesosuchus browni (Reptilia, Archosauromorpha) with information on the inner ear and description of a pneumatic sinus. PeerJ 7:e6798. 10.7717/peerj.6798

Sobral G, Sookias RB, Bhullar B-AS, Smith RMH, Butler RJ, and Müller J. 2016. New information on the braincase and inner ear of Euparkeria capensis Broom: implications for diapsid and archosaur evolution. Royal Society Open Science 3:160072. 10.1098/rsos.160072

Sookias RB. 2016. The relationships of the Euparkeriidae and the rise of Archosauria. Royal Society Open Science 3:150674. 10.1098/rsos.150674

Sookias RB, and Butler RJ. 2013. Euparkeriidae. Geological Society, London, Special Publications 379:3548. 10.1144/SP379.6

Sookias RB, Dilkes D, Sobral G, Smith RM, Wolvaardt FP, Arcucci AB, Bhullar B-AS, and Werneburg I. 2020. The craniomandibular anatomy of the early archosauriform Euparkeria capensis and the dawn of the archosaur skull. Royal Society Open Science 7:200116. 10.1098/rsos.200116

Sookias RB, Sennikov AG, Gower DJ, and Butler RJ. 2014. The monophyly of Euparkeriidae (Reptilia: Archosauriformes) and the origins of Archosauria: a revision of Dorosuchus neoetus from the Mid-Triassic of Russia. Palaeontology 57:1177-1202. 10.1111/pala.12110

Spener CM. 1710. Disquisitio de crocodilo in lapide scissili expresso, aliisque Lithozois. Misc Berol ad increment sci, ex scr Soc Regiae Sci exhibits 1:92-110.

Spiekman SNF. 2018. A new specimen of Prolacerta broomi from the lower Fremouw Formation (Early Triassic) of Antarctica, its biogeographical implications and a taxonomic revision. Scientific Reports 8:17996. 10.1038/s41598-018-36499-6

Spiekman SNF, Bleeker R, Dorst M, de Haan R, Winkelhorst H, and Voeten DFAE. 2019. Tanystropheids from the Winterswijkse Steengroeve - rare but recurring elements in the Vossenveld paleoassemblage. Grondboor \& Hamer (Staringia 16) 73:208-215. 10.5167/uzh-182944

Spiekman SNF, Neenan JM, Fraser NC, Fernandez V, Rieppel O, Nosotti S, and Scheyer TM. 2020a. Aquatic habits and niche partitioning in the extraordinarily long-necked Triassic reptile Tanystropheus. Current Biology 30:1-7. 10.1016/j.cub.2020.07.025

Peer) reviewing PDF | (2020:08:51657:1:1:NEW 28 Feb 2021) 
5034

5035

5036

5037

5038

5039

5040

5041

5042

5043

5044

5045

5046

5047

5048

5049

5050

5051

5052

5053

5054

5055

5056

5057

5058

5059

5060

5061

5062

5063

5064

5065

5066

5067

5068

5069

5070

5071

5072

5073

5074

5075

5076

5077

5078

5079
Spiekman SNF, Neenan JM, Fraser NC, Fernandez V, Rieppel O, Nosotti S, and Scheyer TM. 2020b. The cranial morphology of Tanystropheus hydroides (Tanystropheidae, Archosauromorpha) as revealed by synchrotron microtomography. PeerJ 8:e10299. 10.7717/peerj.10299

Spiekman SNF, and Scheyer TM. 2019. A taxonomic revision of the genus Tanystropheus (Archosauromorpha, Tanystropheidae). Palaeontologia Electronica 22:1-46. 10.26879/1038

Spielmann JA, Heckert AB, and Lucas SG. 2005. The Late Triassic archosauromorph Trilophosaurus as an arboreal climber. Rivista Italiana di Paleontologia e Stratigrafia 111:395-312. 10.13130/2039$4942 / 6328$

Spielmann JA, Lucas SG, Hunt AP, and Heckert AB. 2006. Reinterpretation of the holotype of Malerisaurus langstoni, a diapsid reptile from the Upper Triassic Chinle Group of West Texas. New Mexico Museum Natural History and Science Bulletin 37:543-547.

Spielmann JA, Lucas SG, Rinehart LF, and Heckert AB. 2008. The Late Triassic archosauromorph Trilophosaurus. New Mexico Museum Natural History and Science Bulletin 43:1-177.

Stockar R. 2010. Facies, depositional environment, and palaeoecology of the Middle Triassic Cassina beds (Meride Limestone, Monte San Giorgio, Switzerland). Swiss Journal of Geosciences 103:101-119. 10.1007/s00015-010-0008-2

Stocker MR, Zhao L-J, Nesbitt SJ, Wu X-C, and Li C. 2017. A short-snouted, Middle Triassic phytosaur and its implications for the morphological evolution and biogeography of Phytosauria. Scientific Reports 7:46028. 10.1038/srep46028

Sues H-D, and Olsen PE. 2015. Stratigraphic and temporal context and faunal diversity of Permian-Jurassic continental tetrapod assemblages from the Fundy rift basin, eastern Canada. Atlantic Geology 51:139-205. 10.4138/atlgeol.2015.006

Sullivan C, Reisz RR, and May WJ. 2000. Large dissorophoid skeletal elements from the Lower Permian Richards Spur fissures, Oklahoma, and their paleoecological implications. Journal of Vertebrate Paleontology 20:456-461. 10.1671/0272-4634(2000)020[0456:LDSEFT]2.0.CO;2

Sun Z, Jiang D, Ji C, and Hao W. 2016. Integrated biochronology for Triassic marine vertebrate faunas of Guizhou Province, South China. Journal of Asian Earth Sciences 118:101-110. 10.1016/j.jseaes.2016.01.004

Szulc J, Racki G, and Bodzioch A. 2017. Comment on "An early Late Triassic long-necked reptile with a bony pectoral shield and gracile appendages" by Jerzy Dzik and Tomasz Sulej. Acta Palaeontologica Polonica 62:287-288. 10.4202/app.00352.2017

Tackett LS, and Tintori A. 2019. Low drilling frequency in Norian benthic assemblages from the southern Italian Alps and the role of specialized durophages during the Late Triassic. Palaeogeography, Palaeoclimatology, Palaeoecology 513:25-34. 10.1016/j.palaeo.2018.06.034

Tatarinov LP. 1978. Triassic Prolacertilians of the USSR. Paleontological Journal 12:88-100.

Tatarinov LP. 1989. "The systematic position and way of life of the problematic Upper Triassic reptile Sharovipteryx mirabilis". Paleontologicheskii Zhurnal 2:110-112 (in Russian).

Tatarinov LP. 1994. Terrestrial vertebrates from the Triassic of the USSR with comments on the morphology of some reptiles. In Evolution, Ecology and Biogeography of the Triassic Reptiles, by Mazin, J-M and Pinna, G, eds Paleontologia Lombarda, New Series, 2:165-170.

Trotteyn MJ, and Ezcurra MD. 2014. Osteology of Pseudochampsa ischigualastensis gen. et comb. nov. (Archosauriformes: Proterochampsidae) from the early Late Triassic Ischigualasto Formation of northwestern Argentina. PLOS ONE 9:e111388. 10.1371/journal.pone.0111388

Tverdokhlebov VP, Tverdokhlebova GI, Surkov MV, and Benton MJ. 2003. Tetrapod localities from the Triassic of the SE of European Russia. Earth-Science Reviews 60:1-66. 10.1016/S00128252(02)00076-4

PeerJ reviewing PDF | (2020:08:51657:1:1:NEW 28 Feb 2021) 
5080

5081

5082

5083

5084

5085

5086

5087

5088

5089

5090

5091

5092

5093

5094

5095

5096

5097

5098

5099

5100

5101

5102

5103

5104

5105

5106

5107

5108

5109

5110

5111

5112

5113

5114

5115

5116

5117

5118

5119

5120

5121

5122

5123

5124

5125

Unwin DM, Alifanov VR, and Benton MJ. 2000. Enigmatic small reptiles from the Middle-Late Triassic of Kirgizstan. In The age of dinosaurs in Russia and Mongolia, by Benton M J, Kurochkin E N, Shishkin M A, Unwin D M, eds Cambridge: Cambridge University Press:140-159.

Vaughn PP. 1955. The Permian reptile Araeoscelis restudied. Bulletin of the Museum of Comparative Zoology at Harvard College 113:303-469.

Walker AD. 1969. The reptile fauna of the 'Lower Keuper' Sandstone. Geological Magazine 106:470-476. $10.1017 /$ S0016756800058842

Watson DMS. 1912. Mesosuchus browni, gen. et spec. nov. Records of the Albany Museum 2:298-299.

Watson DMS. 1917. A sketch classification of the pre-Jurassic tetrapod vertebrates. Proceedings of the Zoological Society of London 87:167-186. 10.1111/j.1096-3642.1917.tb02055.x

Watson DMS. 1957. On Millerosaurus and the early history of the sauropsid reptiles. Philosophical Transactions of the Royal Society of London B: Biological Sciences 240:325-400. 10.1098/rstb.1957.0003

Welman J. 1998. The taxonomy of the South African proterosuchids (Reptilia, Archosauromorpha). Journal of Vertebrate Paleontology 18:340-347. 10.1080/02724634.1998.10011062

Werning S, and Irmis R. 2010. Reconstructing the ontogeny of the Triassic basal archosauromorph Trilophosaurus using bone histology and limb bone morphometrics. SVP Program and Abstracts 2010:185-186. Whiteside DI, and Duffin CJ. 2017. Late Triassic terrestrial microvertebrates from Charles Moore's 'Microlestes' quarry, Holwell, Somerset, UK. Zoological Journal of the Linnean Society 179:677-705. 10.1111/zoj.12458

Whiteside DI, Duffin CJ, Gill PG, Marshall JEA, and Benton MJ. 2016. The Late Triassic and Early Jurassic fissure faunas from Bristol and South Wales: stratigraphy and setting. Palaeontologia Polonica 67:257-287. 10.4202/pp.2016.67_257

Whiteside DI, and Marshall JE. 2008. The age, fauna and palaeoenvironment of the Late Triassic fissure deposits of Tytherington, South Gloucestershire, UK. Geological Magazine 145:105-147. 10.1017/S0016756807003925

Wild R. 1973. Die Triasfauna der Tessiner Kalkalpen XXII. Tanystropheus longobardicus (Bassani) (Neue Ergebnisse). Schweizerische Paläontologische Abhandlungen 95:1-182.

Wild R. 1980a. Neue Funde von Tanystropheus (Reptilia, Squamata). Schweizerische Paläontologische Abhandlungen 102.

Wild R. 1980b. Tanystropheus (Reptilia : Squamata) and its importance for stratigraphy. Mémoires de la Société Géologique de France, n s 139:201-206.

Wild R. 1987. An example of biological reasons for extinction: Tanystropheus (Reptilia, Squamata). Mémoires de la Société Géologique de France, n s 150:37-44.

Wild R. 1991. Aetosaurus (Reptilia, Thecodontia) from the Upper Triassic (Norian) of Cene near Bergamo, Italy, with a revision of the genus. Rivista Italiana di Paleontologia e Stratigrafia Museo Civico di Scienze Naturali "E Caffi" Bergamo 14:1-24.

Wild R, and Oosterink H. 1984. Tanystropheus (Reptilia: Squamata) aus dem Unteren Muschelkalk von Winterswijk, Holland. Grondboor \& Hamer 38:142-148.

Williston SW. 1925. Osteology of the Reptiles. Cambridge: Harvard University Press. 300 p.Witmer LM. 1997. Craniofacial air sinus systems. In: Currie PJ, and Padian K, eds. Encyclopedia of dinosaurs. New York: Academic Press, 151-159.

Woodhead J, Reisz R, Fox D, Drysdale R, Hellstrom J, Maas R, Cheng H, and Edwards RL. 2010. Speleothem climate records from deep time? Exploring the potential with an example from the Permian. Geology 38:455-458. 10.1130/G30354.1

Young C-C. 1973. The discovery of Prolacertilia in Jimusar, Sinkiang. Vertebrata PalAsiatica 11:46-49.

PeerJ reviewing PDF | (2020:08:51657:1:1:NEW 28 Feb 2021) 


\section{Table $\mathbf{1}$ (on next page)}

Percentage of scored characters of each OTU for the character matrix used in this study. 
1 Table 1. Percentage of scored characters of each OTU for the character matrix used in this study.

\begin{tabular}{|c|c|}
\hline OTU & $\%$ of characters scored \\
\hline Petrolacosaurus kansensis & 87.3 \\
\hline Orovenator mayorum & 47.6 \\
\hline Youngina capensis & 90.6 \\
\hline Acerosodontosaurus piveteaui & 31.6 \\
\hline Claudiosaurus germaini & 64.2 \\
\hline Gephyrosaurus bridensis & 78.2 \\
\hline Planocephalosaurus robinsonae & 75.6 \\
\hline Czatkowiella harae & 50.8 \\
\hline Protorosaurus speneri & 73.3 \\
\hline Jesairosaurus lehmani & 54.7 \\
\hline Macrocnemus bassanii & 93.2 \\
\hline Macrocnemus obristi & 8.1 \\
\hline Macrocnemus fuyuanensis & 71.3 \\
\hline Tanystropheus hydroides & 91.9 \\
\hline GMPKU P1527 T. cf. hydroides & 24.4 \\
\hline Tanystropheus longobardicus & 79.8 \\
\hline Tanystropheus conspicuus & 4.6 \\
\hline "Tanystropheus antiquus" & 5.2 \\
\hline Sclerostropheus fossai & 4.9 \\
\hline Raibliania calligarisi & 9.4 \\
\hline Augustaburiania vatagini & 8.8 \\
\hline Langobardisaurus pandolfii & 49.5 \\
\hline Amotosaurus rotfeldensis & 45.9 \\
\hline AMNH FARB 7206 & 6.2 \\
\hline Tanytrachelos ahynis & 37.1 \\
\hline Ozimek volans & 31.3 \\
\hline Elessaurus gondwanoccidens & 8.1 \\
\hline Pectodens zhenyuensis & 45.6 \\
\hline Fuyuansaurus acutirostris & 25.7 \\
\hline Dinocephalosaurus orientalis & 64.8 \\
\hline Prolacerta broomi & 96.7 \\
\hline Pamelaria dolichotrachela & 59.3 \\
\hline Azendohsaurus madagaskarensis & 94.5 \\
\hline Trilophosaurus buettneri & 89.3 \\
\hline Mesosuchus browni & 89.3 \\
\hline Howesia browni & 46.6 \\
\hline Eohyosaurus wolvaardti & 25.7 \\
\hline Teyujagua paradoxa & 47.9 \\
\hline Proterosuchus fergusi & 56.0 \\
\hline Proterosuchus alexanderi & 74.9 \\
\hline Euparkeria capensis & 90.2 \\
\hline Erythrosuchus africanus & 84.4 \\
\hline
\end{tabular}


Figure 1

Selected phylogenetic hypotheses for "protorosaur" relationships.

(A) Ezcurra 2016. (B) Pritchard et al. 2015. (C) Rieppel et al. 2003, which represents a compilation of the matrices of Benton \& Allen (1997), Jalil (1997), and Dilkes (1998). 


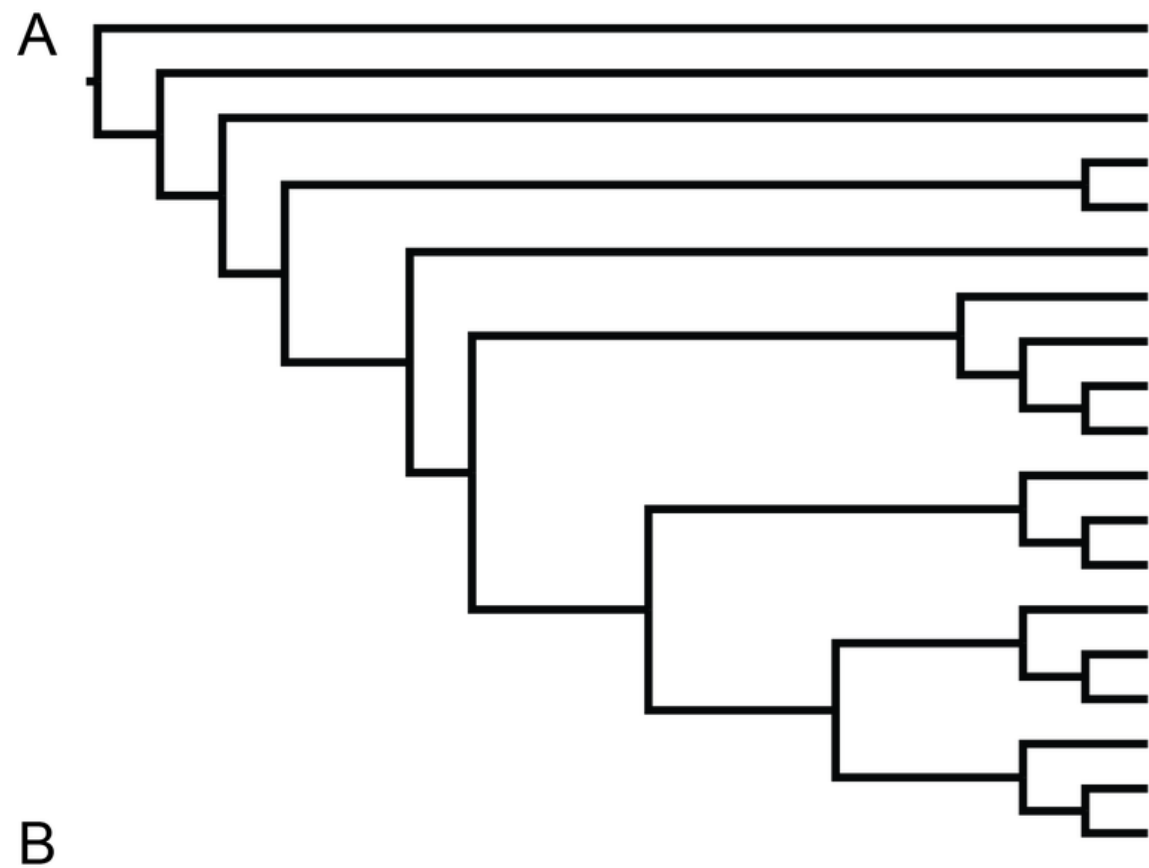

Petrolacosaurus kansensis

Youngina capensis

Choristodera

Planocephalosaurus robinsonae

Gephyrosaurus bridensis

Protorosaurus speneri

Jesairosaurus lehmani

Macrocnemus bassanii

Tanystropheus longobardicus

Amotosaurus rotfeldensis

Pamelaria dolichotrachela

Trilophosaurus buettneri

- Azendohsaurus madagaskansis

- Mesosuchus browni

Howesia browni

Rhynchosaurus articeps

Boreopricea funerea

- Prolacerta broomi

Archosauriformes

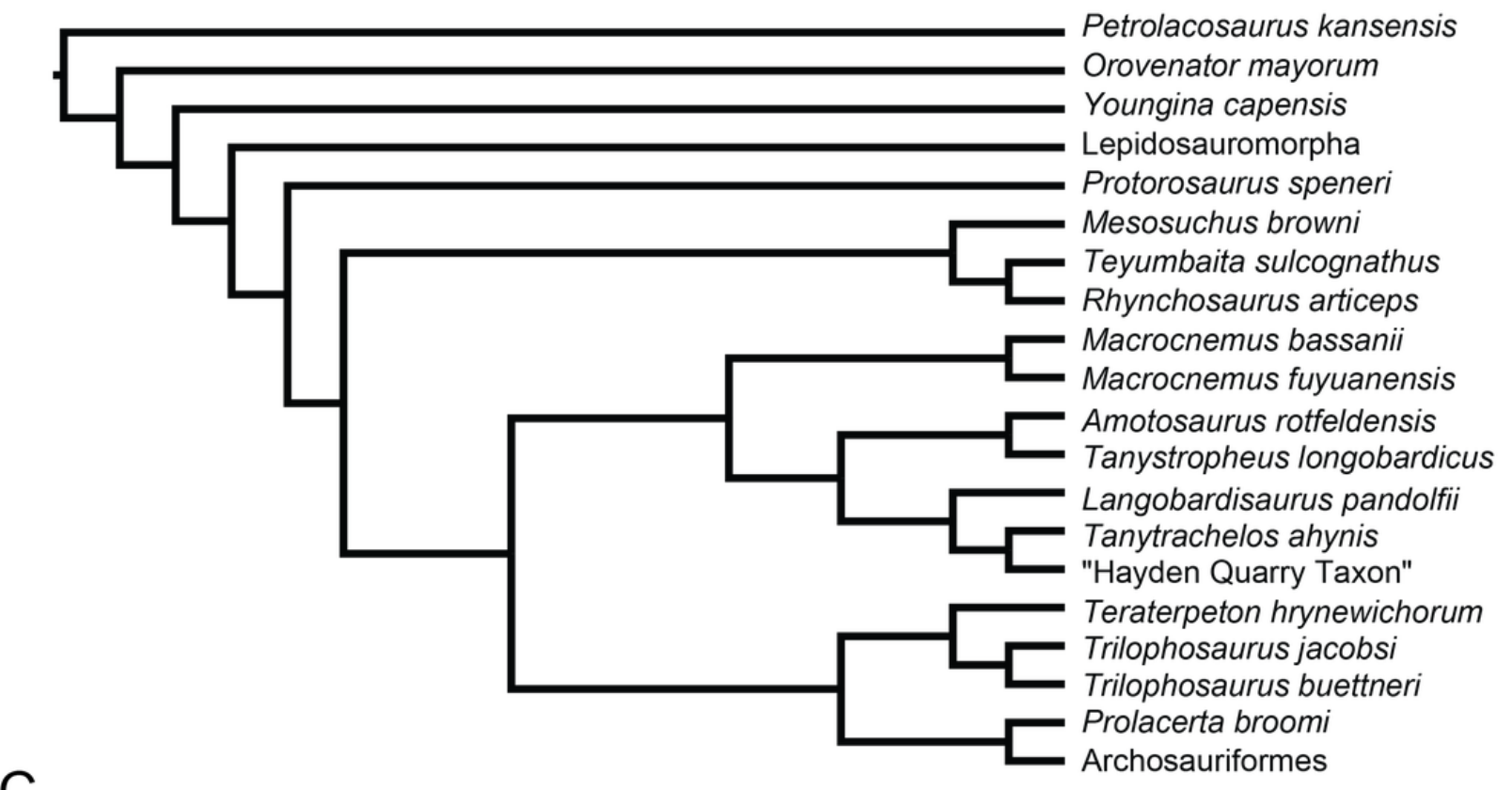

C

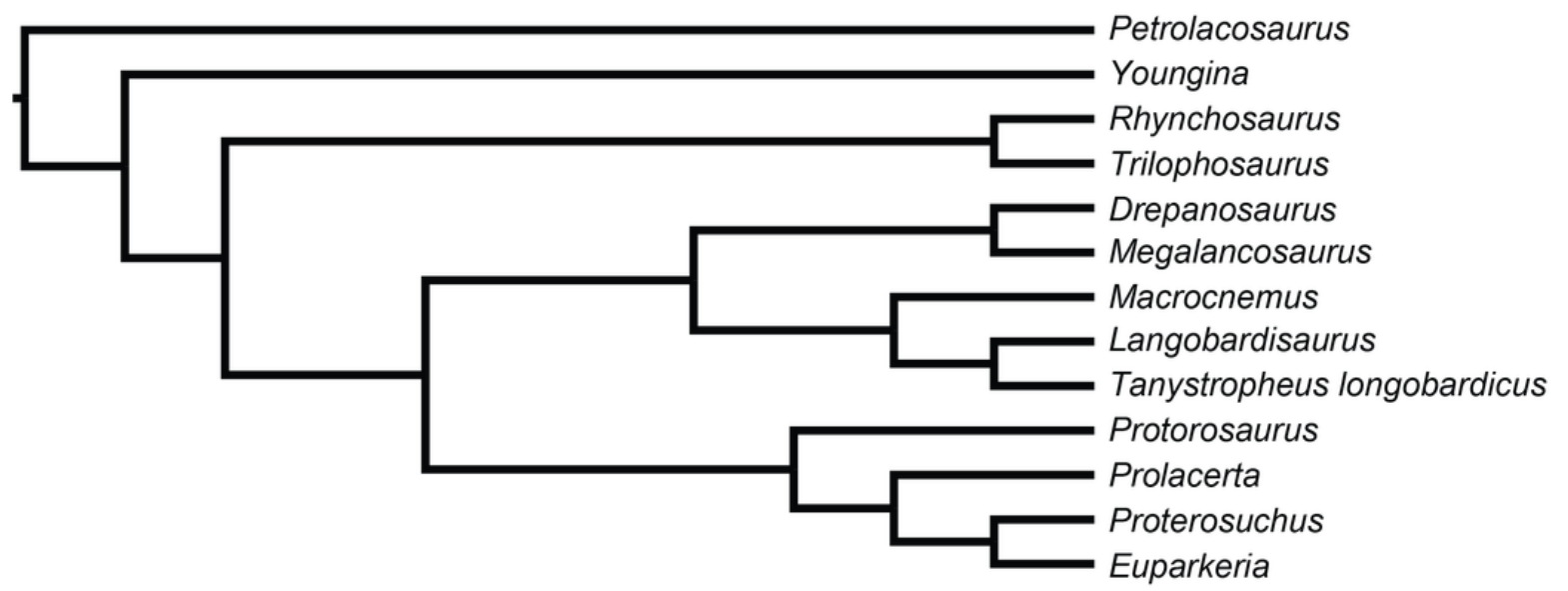


Figure 2

Illustration of character 17.

(A) State 0 in Prolacerta broomi (BP/1/5880, anterior snout in left lateral view). (B) State 1 in a digital reconstruction of Tanystropheus hydroides (PIMUZ T 2790, anterior snout in left lateral view). (C) State 3 in Erythrosuchus africanus (BP/1/4526, isolated right premaxilla in lateral view). (D) State 4 in a digital reconstruction of Macrocnemus bassanii (PIMUZ T 2477, anterior snout in right lateral and medial view). 

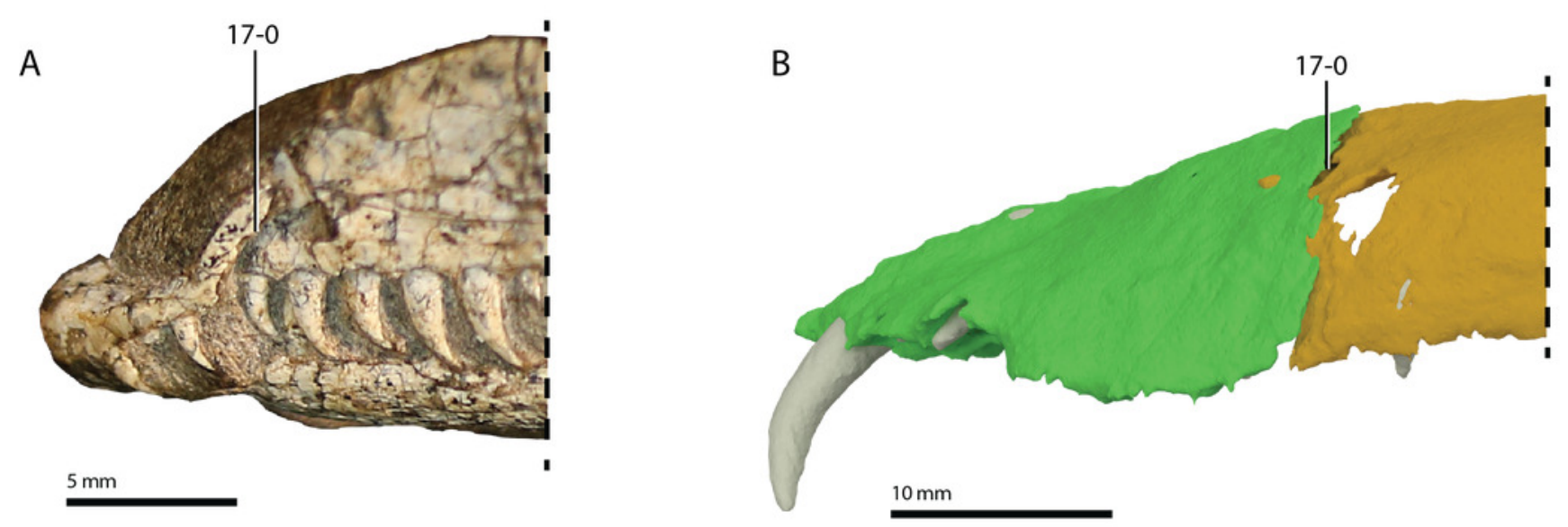

C
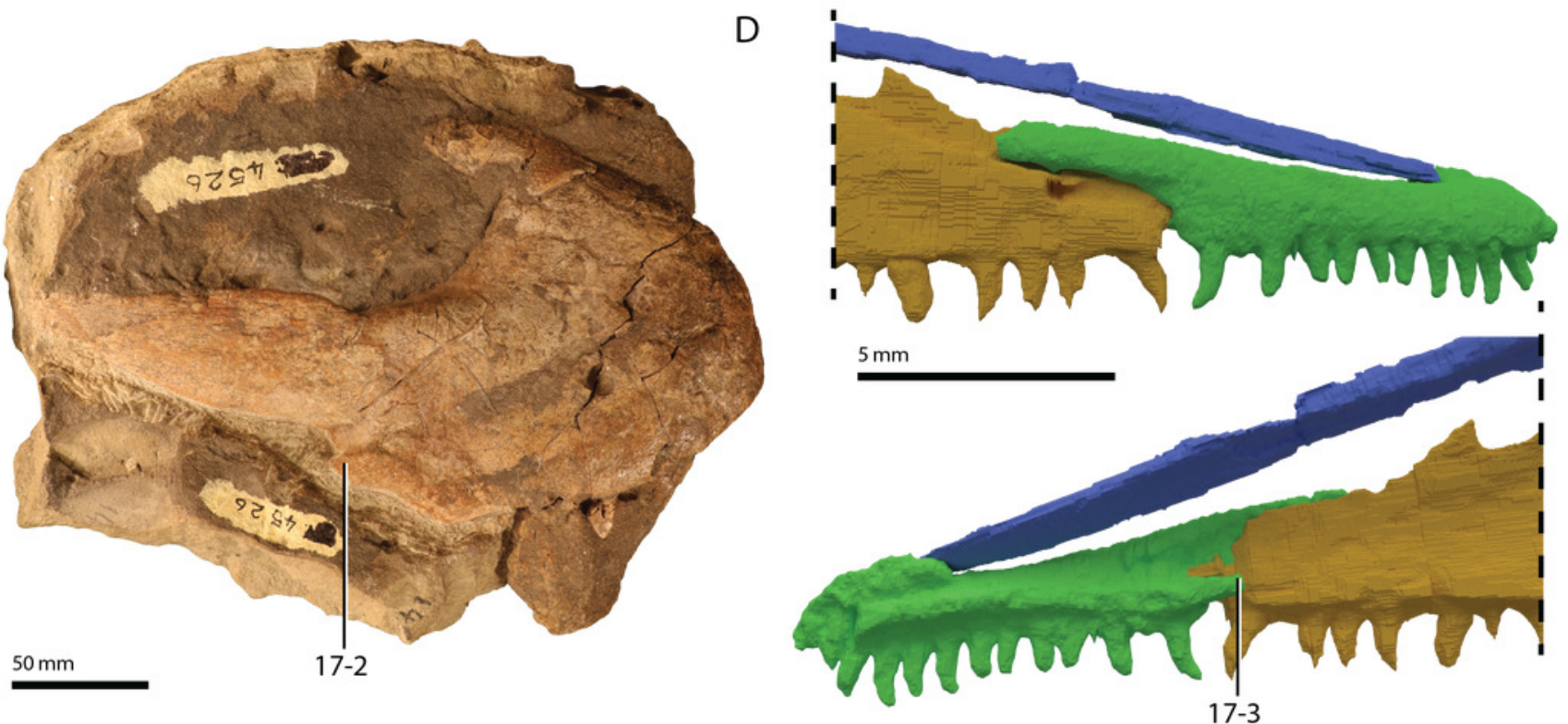


\section{Figure 3}

Illustration of character 20.

(A) State 0 in Protorosaurus speneri (NMK S 180, anterior part of the skull in right laterodorsal view). (B) State 1 in Amotosaurus rotfeldensis (SMNS 50830, right maxilla in medial view). (C) State 0 in Youngina capensis (AMNH FARB 5561, skull in right lateral view). (D) State 1 in Macrocnemus fuyuanensis (PIMUZ T 1559, disarticulated skull with right maxilla visible in lateral view). The stippled line indicates the transition from the dorsal to the posterior margin of the maxilla in $(A)$ and $(B)$.

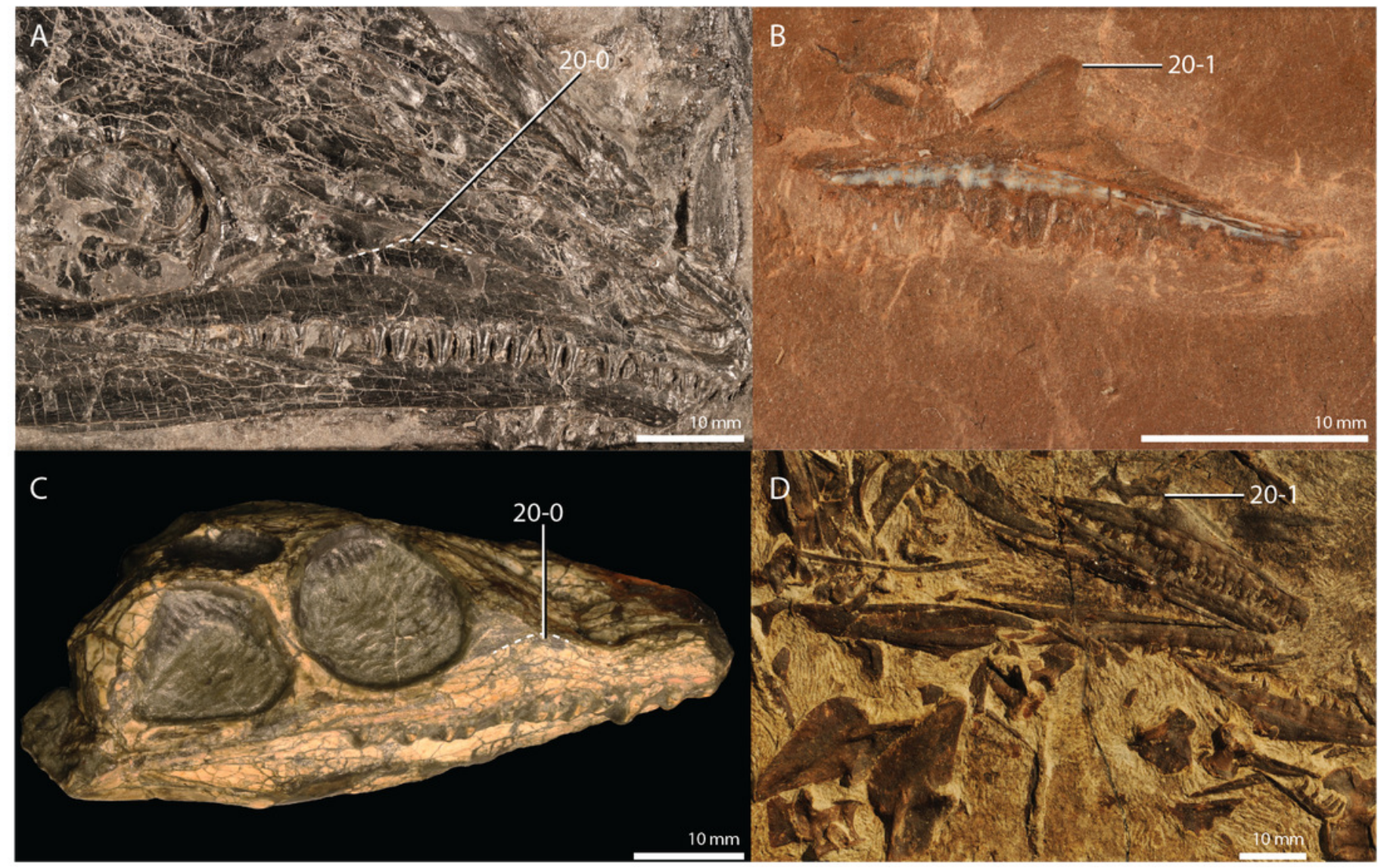




\section{Figure 4}

Illustration of character 23.

(A) State 0 in Macrocnemus bassanii (PIMUZ T 4822, skull in left lateral view). (B) State 1 in Dinocephalosaurus orientalis (IVPP V13767, anterior part of the skull in right laterodorsal view). (C) Close up of the maxillary fossa on the right maxilla of Dinocephalosaurus orientalis (IVPP V13767).

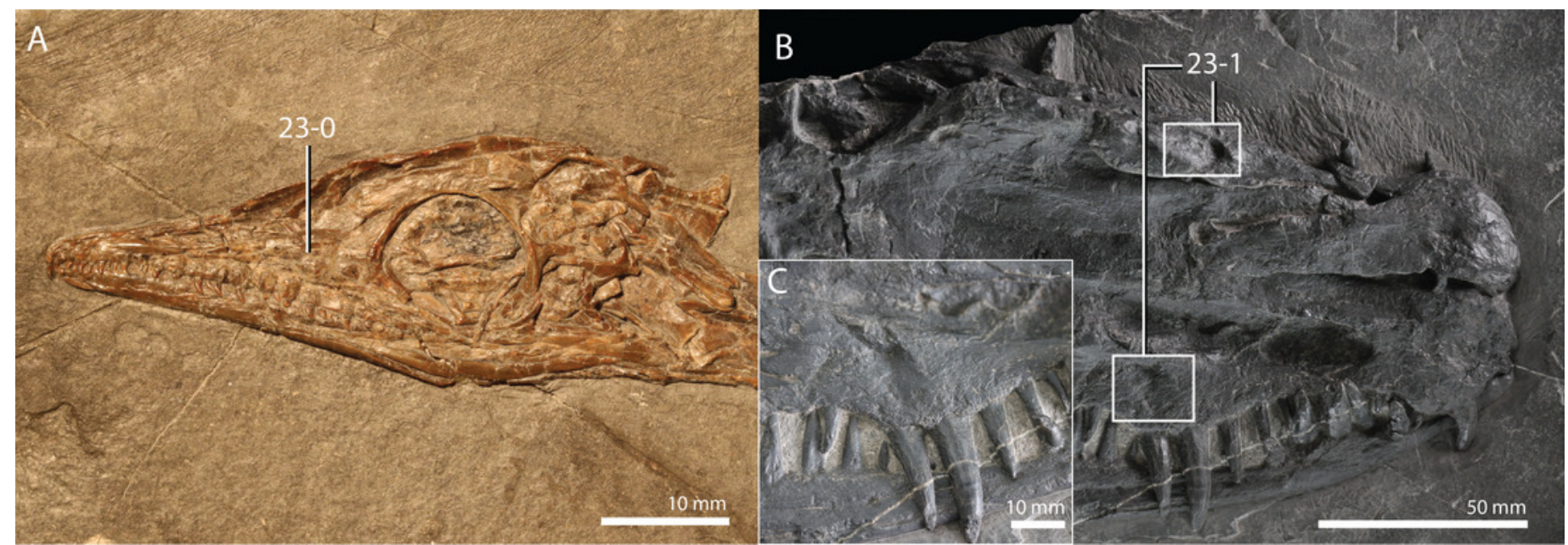




\section{Figure 5}

Illustration of character 25.

(A) State 0 in a digital reconstruction of Tanystropheus hydroides (PIMUZ T 2790, skull in right lateral view). (B) State 1 in a digital reconstruction of Macrocnemus bassanii (PIMUZ T 2477, skull in left lateral view). (C) State 2 in Euparkeria capensis (SAM-PK-5867, skull in right lateral view). (D) State 4 in Dinocephalosaurus orientalis (IVPP V13767, skull in right dorsolateral view).

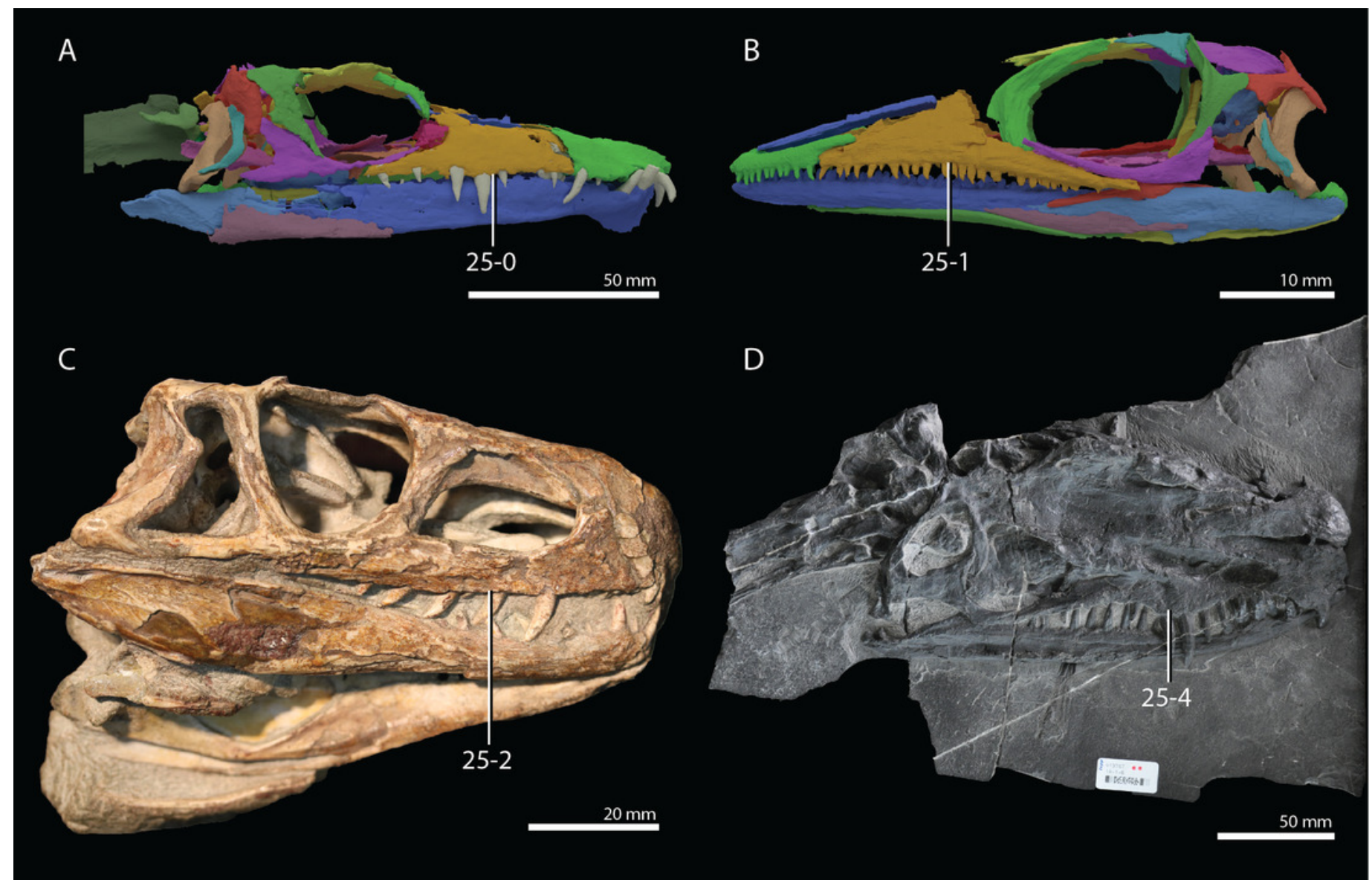




\section{Figure 6}

Illustration of character 31.

(A) State 0 in Youngina capensis (SAM-PK-K7578, skull in dorsal view). (B) State 1 in Pectodens zhenyuensis (IVPP V18578, skull in right lateral view).

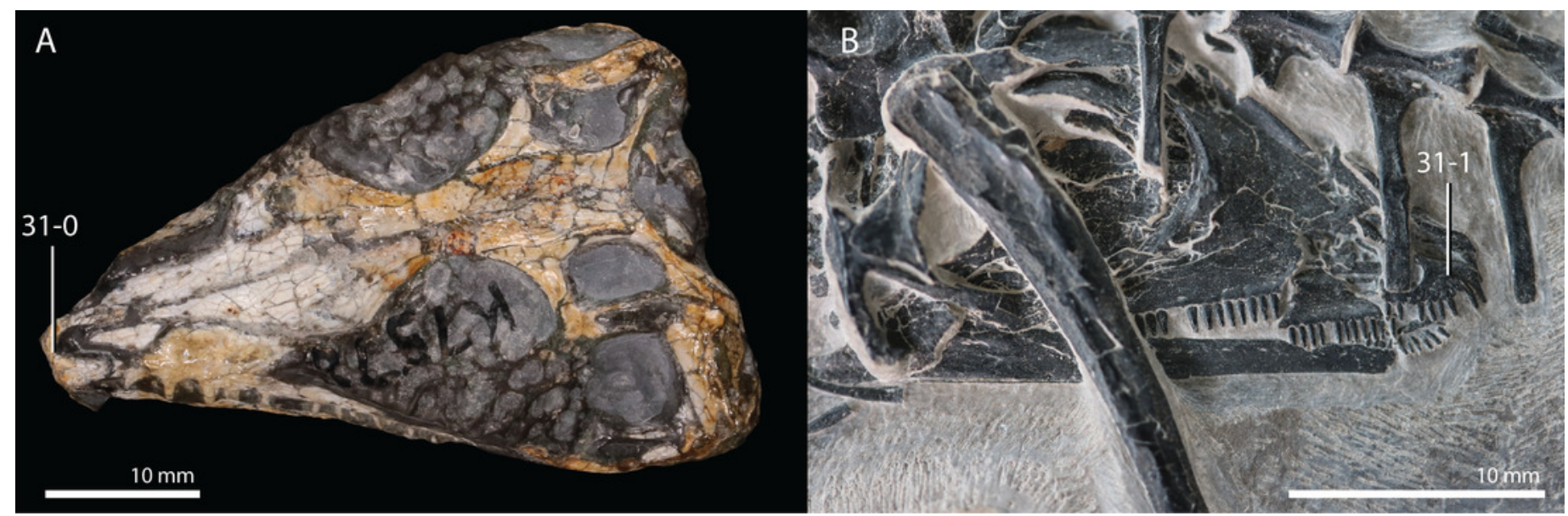




\section{Figure 7}

Illustration of character 33.

(A) State 1 in Dinocephalosaurus orientalis (IVPP V13767, anterior part of the skull in right dorsolateral view). (B) State 1 in Tanystropheus hydroides (PIMUZ T 2819, anterior part of the skull in dorsal view). (C) State 0 in Mesosuchus browni (SAM-PK-6536, skull in dorsal view). (D) State 0 in Euparkeria capensis (SAM-PK-5867, skull in dorsal view).

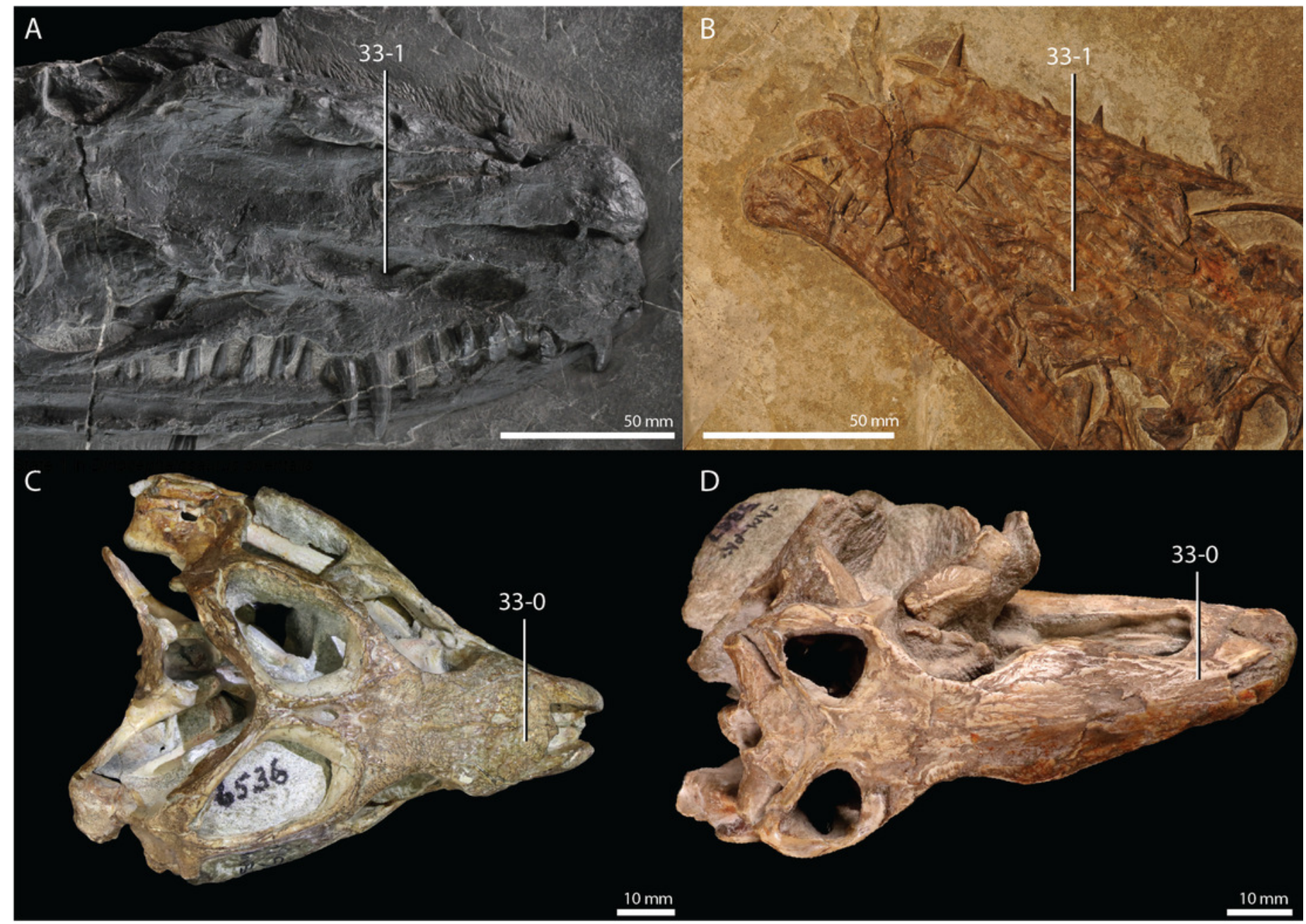




\section{Figure 8}

Illustration of characters 38 and 39.

(A) 38-1 and 39-0 in Euparkeria capensis (SAM-PK-5867, skull in right lateral view). (B) 38-1 and 39-1 in Erythrosuchus africanus (BP/1/5207, skull in right lateral view). (C) 38-0 and 39-1 in Proterosuchus alexanderi (NM QR 1484, skull in right lateral view). Image of Proterosuchus alexanderi courtesy of Martín Ezcurra.

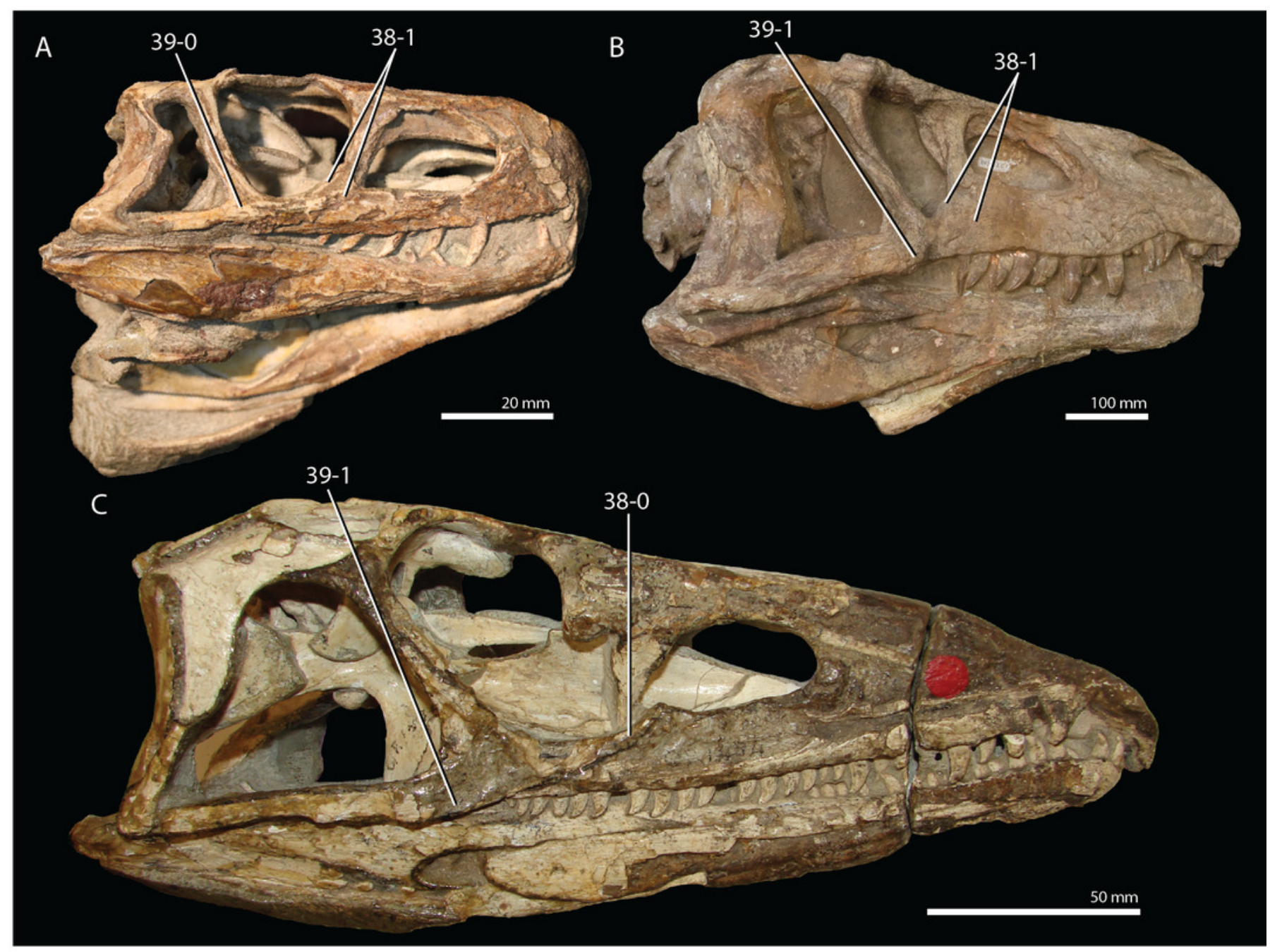


Figure 9

Illustration of characters 42 and 43.

(A) 42-0 and 43-1 in Tanystropheus longobardicus (PIMUZ T 3901, skull in left lateral view).

(B) 42-1 in Dinocephalosaurus orientalis (IVPP V13767, skull in right laterodorsal view).

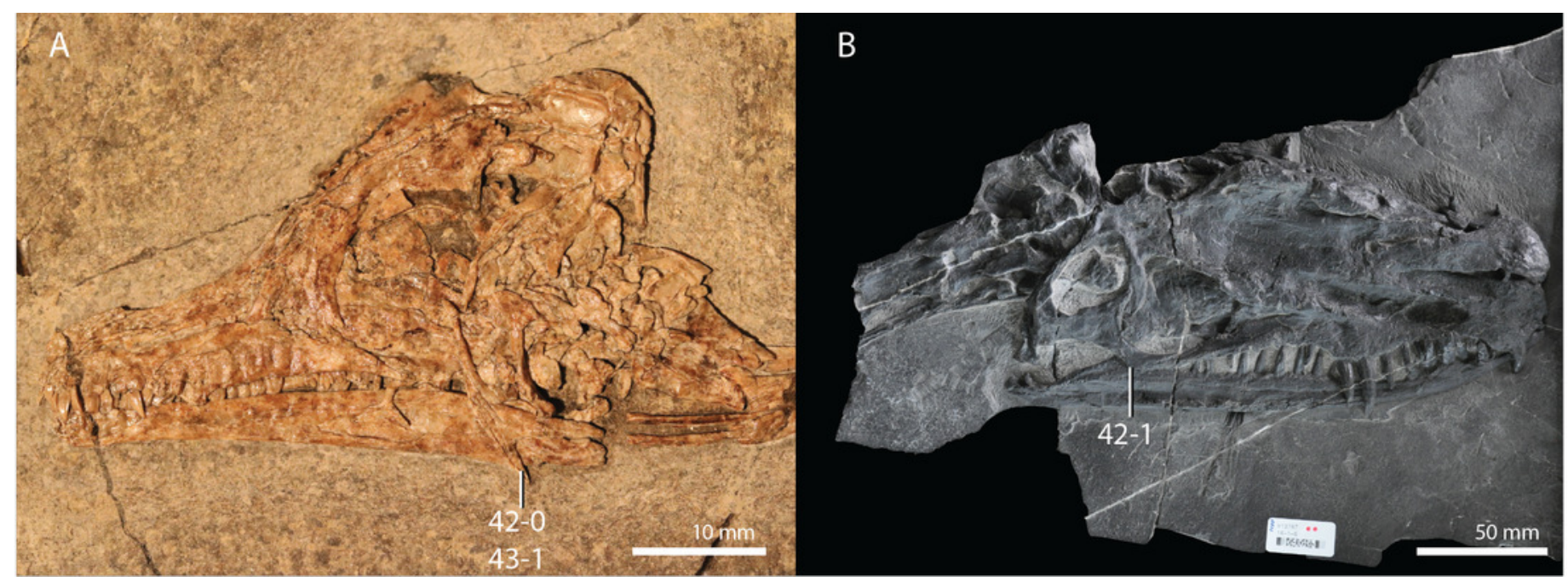




\section{Figure 10}

Illustration of character 49.

(A) State 3 in Youngina capensis (BP/1/3859, partial skull in dorsal view). (B) State 4 in Tanystropheus longobardicus (PIMUZ T 2484, frontal, parietal, and postfrontal in dorsal view).

(C) State 3 in Tanystropheus hydroides (PIMUZ T 2787, frontal and parietal in ventral view).

(D) State 1 in Prolacera broomi (UCMP 37151, skull in dorsal view).

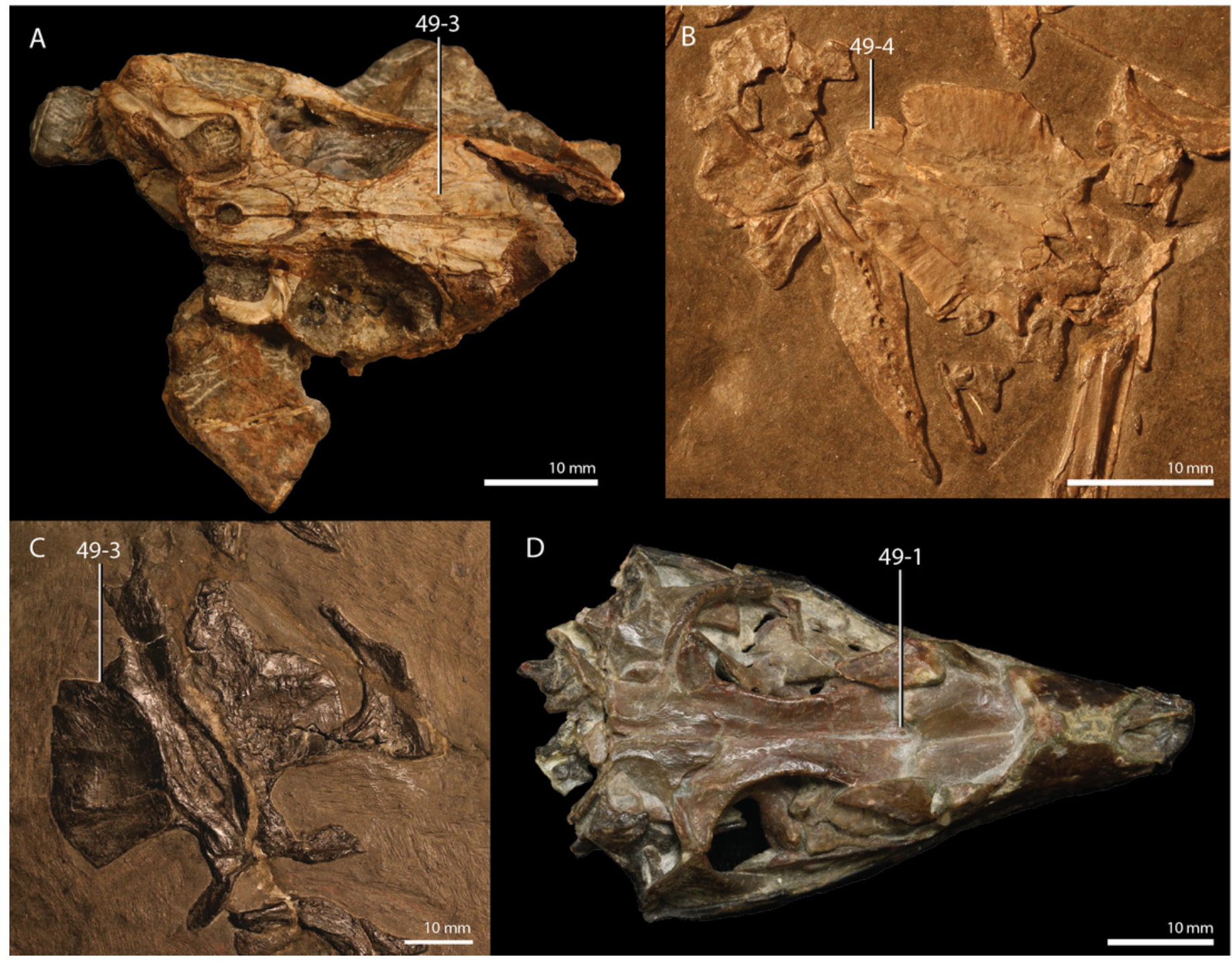




\section{Figure 11}

Illustration of character 55.

(A) State 1 in a digital reconstruction of Tanystropheus hydroides (PIMUZ T 2790, skull in dorsal view). (B) State 1 in Tanystropheus longobardicus (PIMUZ T 2484, frontal, parietal, and postfrontal in dorsal view). (C) State 0 in a digital reconstruction of Macrocnemus bassanii (PIMUZ T 2477, skull in dorsal view). (D) State 0 in Prolacerta broomi (BP/1/471, skull in dorsal view).

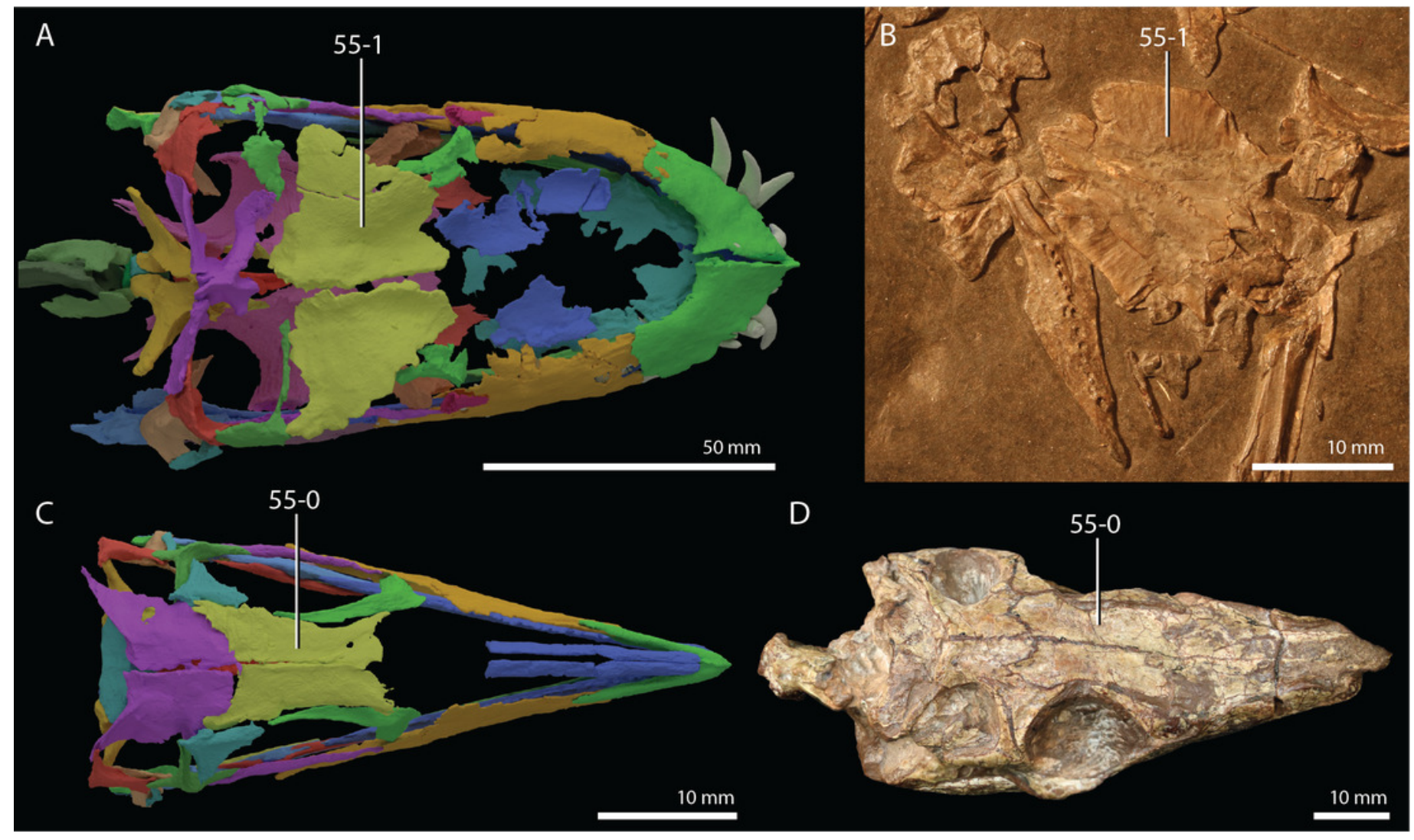




\section{Figure 12}

Illustration of character 57.

(A) State 0 in a digital reconstruction of Macrocnemus bassanii (PIMUZ T 2477, skull in dorsal view). (B) State 1 in Tanystropheus longobardicus (PIMUZ T 2484, frontal, parietal, and postfrontal in dorsal view). (C) State 2 in Proterosuchus alexanderi (NM QR 1484, part of the skull in dorsal view). Image of Proterosuchus alexanderi courtesy of Martín Ezcurra.

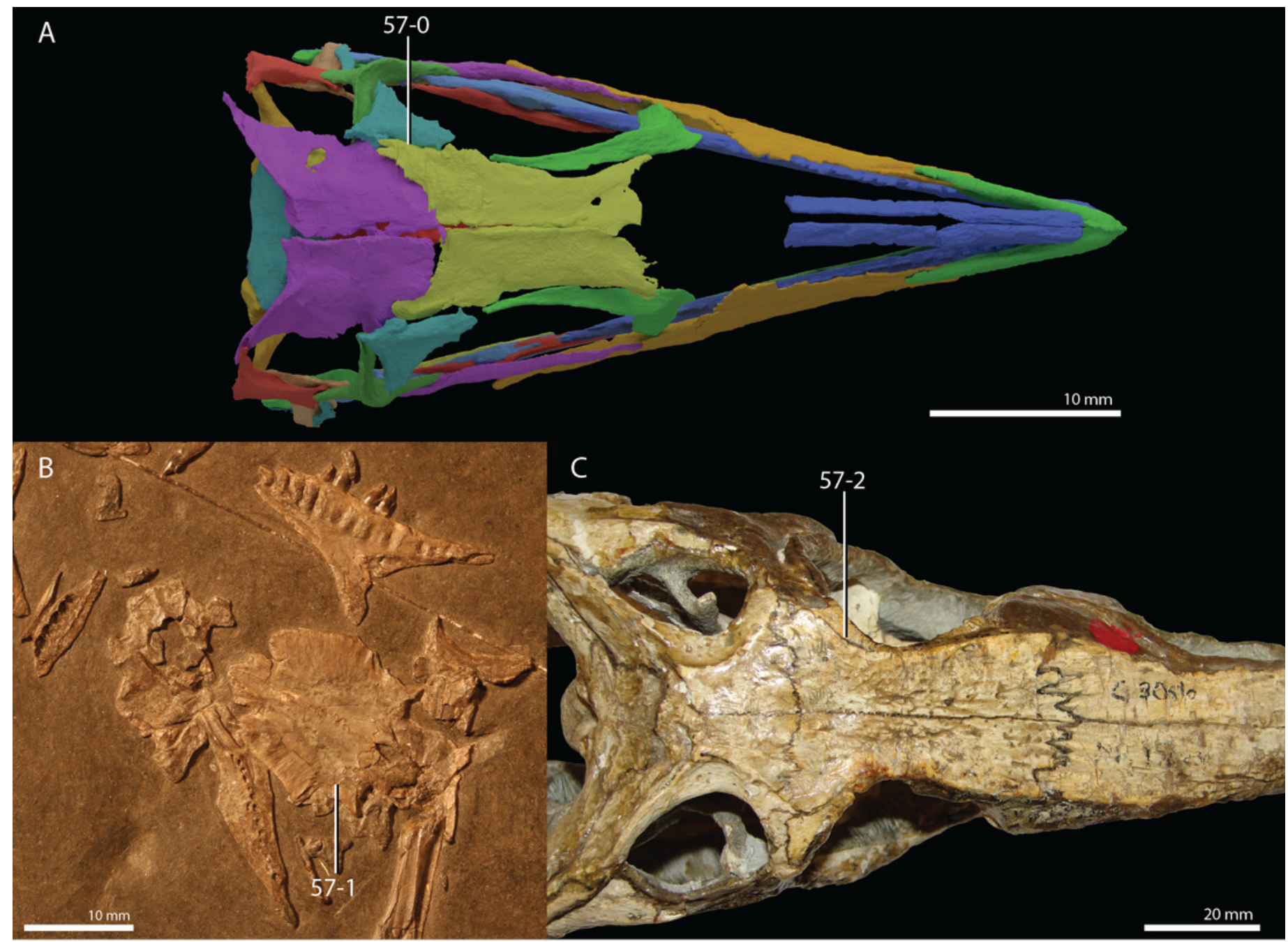




\section{Figure 13}

Illustration of characters 67 and 71.

(A) 67-0 in Prolacerta broomi (BP/1/5375, partial skull in left lateral view). (B) 67-1 in a digital reconstruction of Tanystropheus hydroides (PIMUZ T 2790, posterior part of the skull in right lateral view). (C-D) 67-0 and 71-0 in a digital reconstruction of Macrocnemus bassanii (PIMUZ T 2477, right squamosal in $(C)$ lateral and $(D)$ angled oblique anteroventromedial view). $(E-F)$ 67-1 and 71-1 in a digital reconstruction of Tanystropheus hydroides (PIMUZ T 2790, right squamosal in $(E)$ lateral and $(F)$ anterior view. Abbreviations: ap, anterior process; mp, medial process; pp, posterior process; qf, quadrate facet; $v p$, ventral process. 


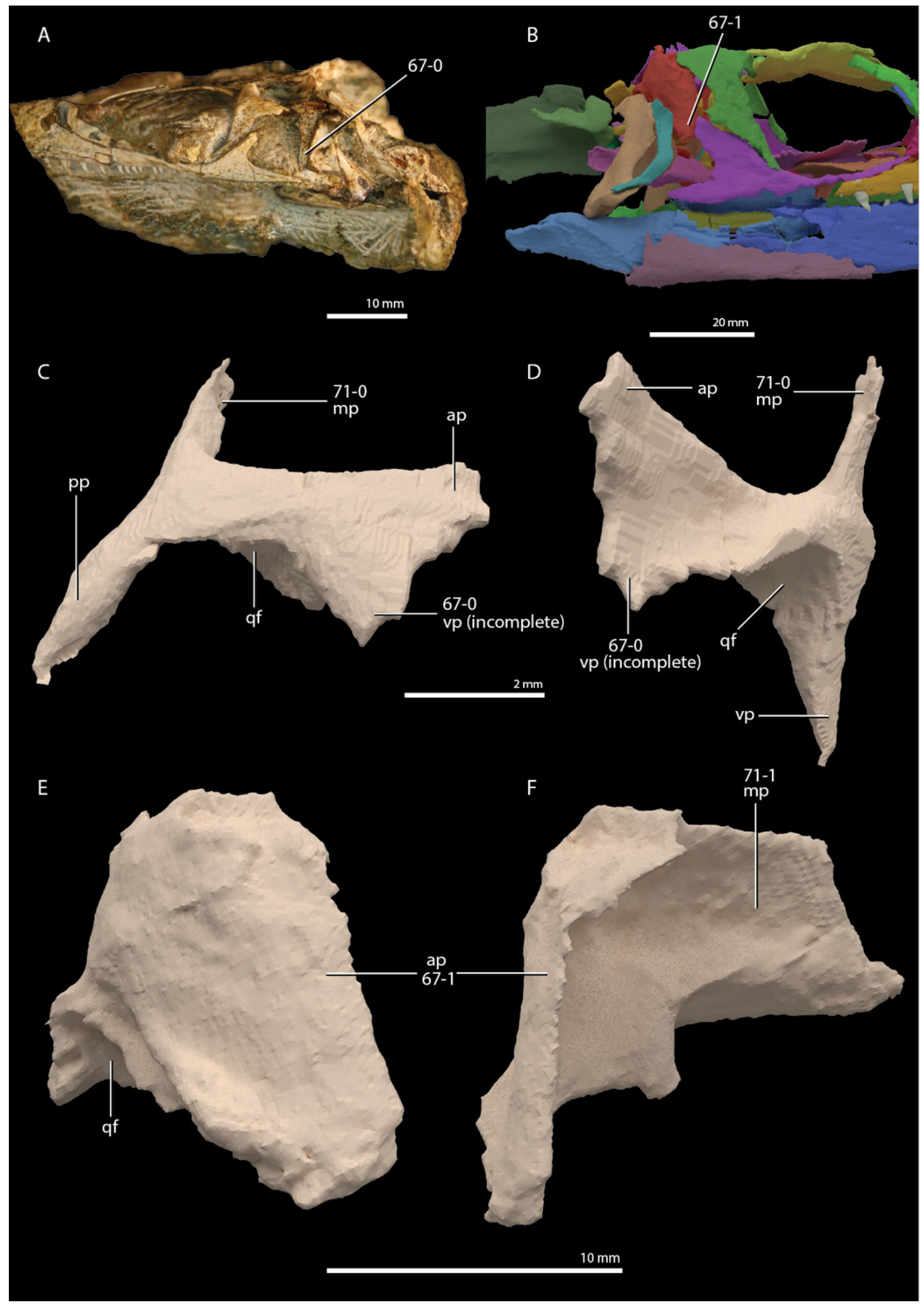




\section{Figure 14}

Illustration of characters $76,79,81$, and 83

(A) 76-0, 79-1, 81-1, 83-0 in Youngina capensis (SAM-PK-K7578, posterior part of the skull in dorsal view). (B) 79-1, 81-0 in a digital reconstruction of Macrocnemus bassanii (PIMUZ T 2477, posterior part of the skull in dorsal view). (C) 76-0, 79-0, 81-1, 83-2 in Tanystropheus hydroides (PIMUZ T 2819, posterior part of the skull in dorsal view). (D) 76-1, 79-1, 81-1, 83-1 in Dinocephalosaurus orientalis (IVPP V13767, posterior part of the skull in right laterodorsal view). (E) 76-1, 79-0, 81-1, 83-1 in Azendohsaurus madagaskarensis (UA-7-20-99-653, partial skull in dorsal view). Image of Azendohsaurus madagaskarensis courtesy of Sterling Nesbitt. 

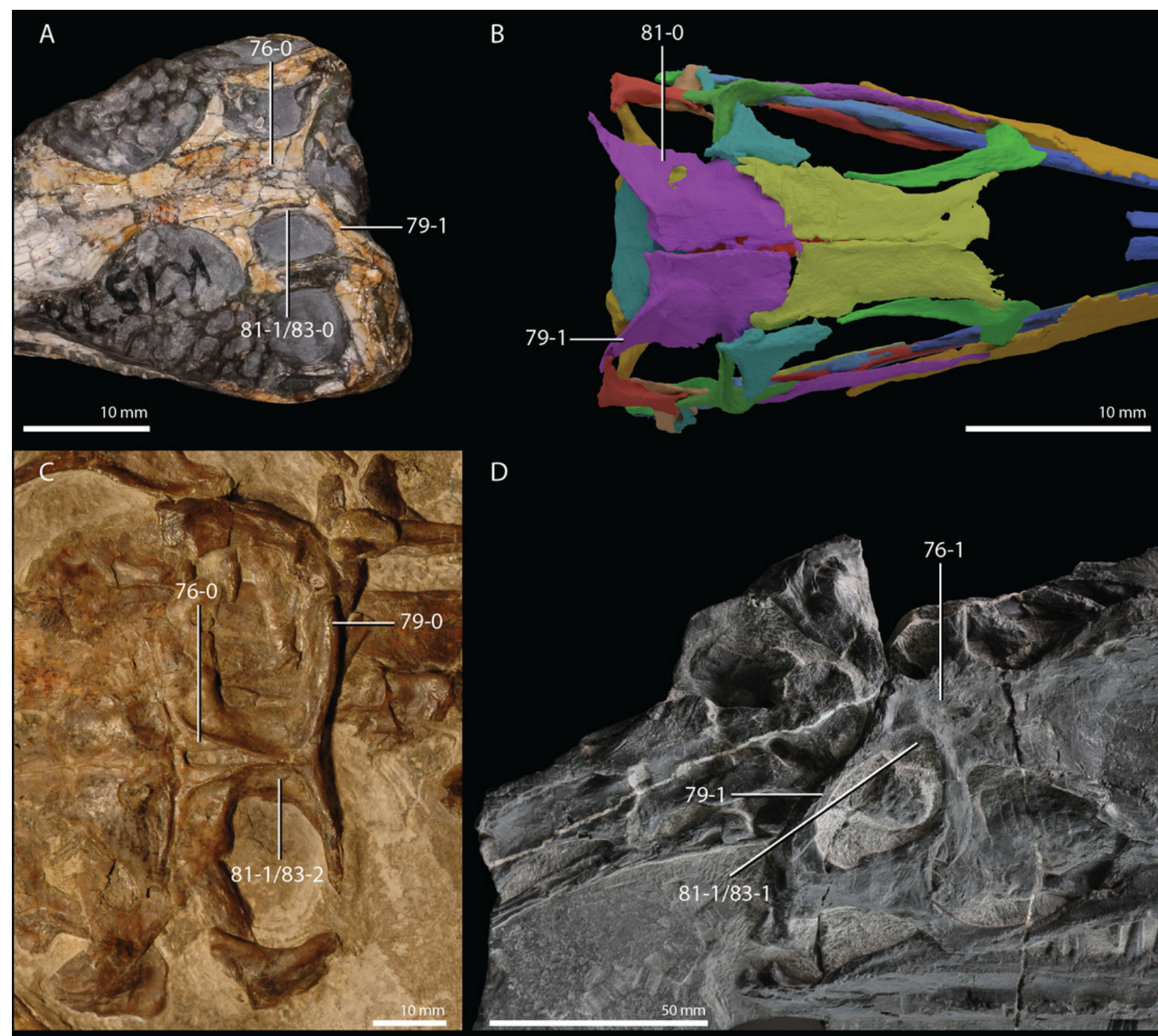

D
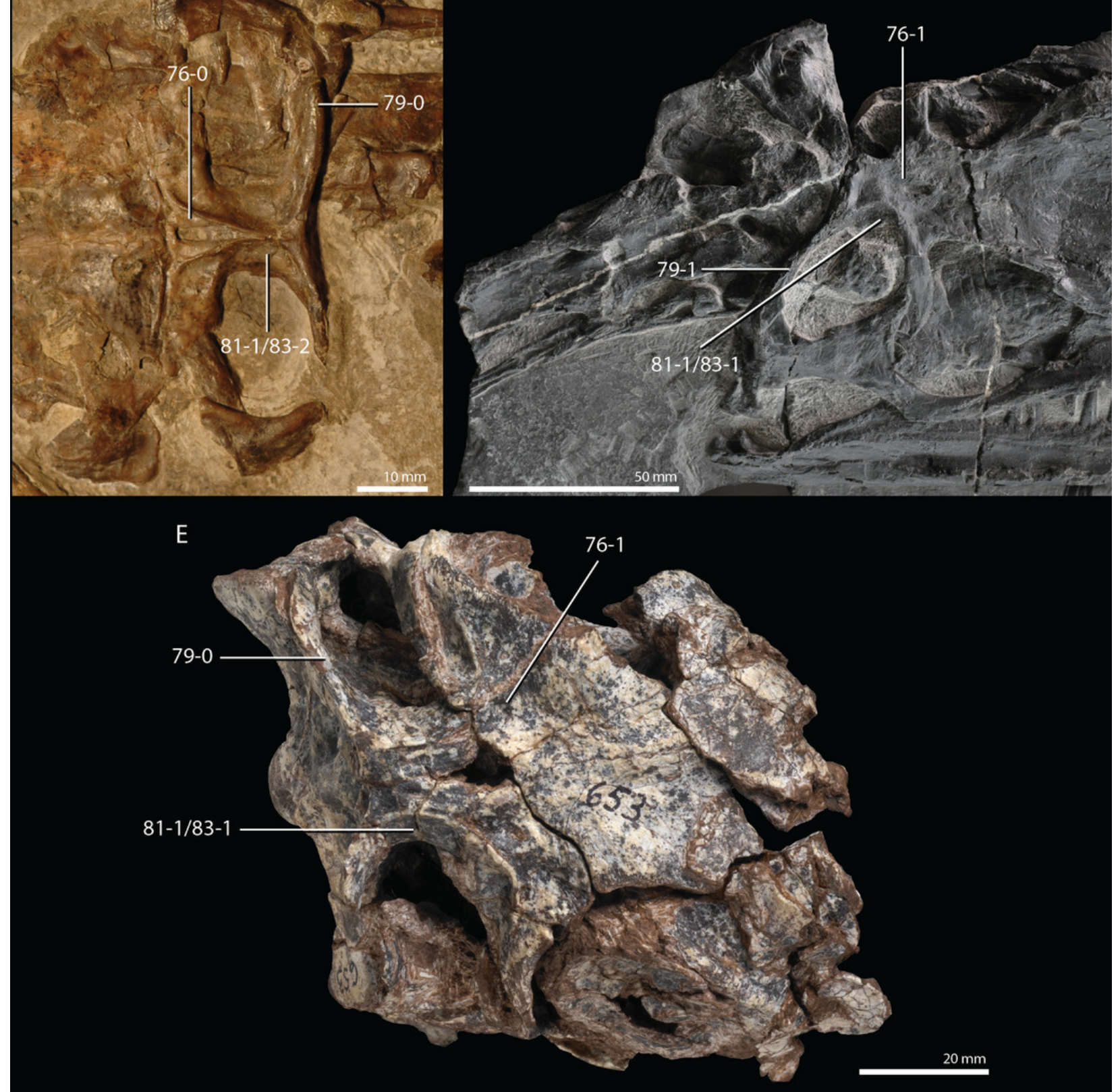


\section{Figure 15}

Illustration of characters 92, 93, and 94 .

(A-B) 92-0, 93-1, 94-1 in a digital reconstruction of Tanystropheus hydroides (PIMUZ T 2790, right quadrate in (A) posterior and (B) medial view). (C) 94-0 in Macrocnemus fuyuanensis (PIMUZ T 1559, right quadrate in anterior view). (D) 92-0 and 93-0 in Proterosuchus alexanderi (NM QR 1484, left side of the skull in posterior/occipital view). Image of Proterosuchus alexanderi courtesy of Martín Ezcurra. 


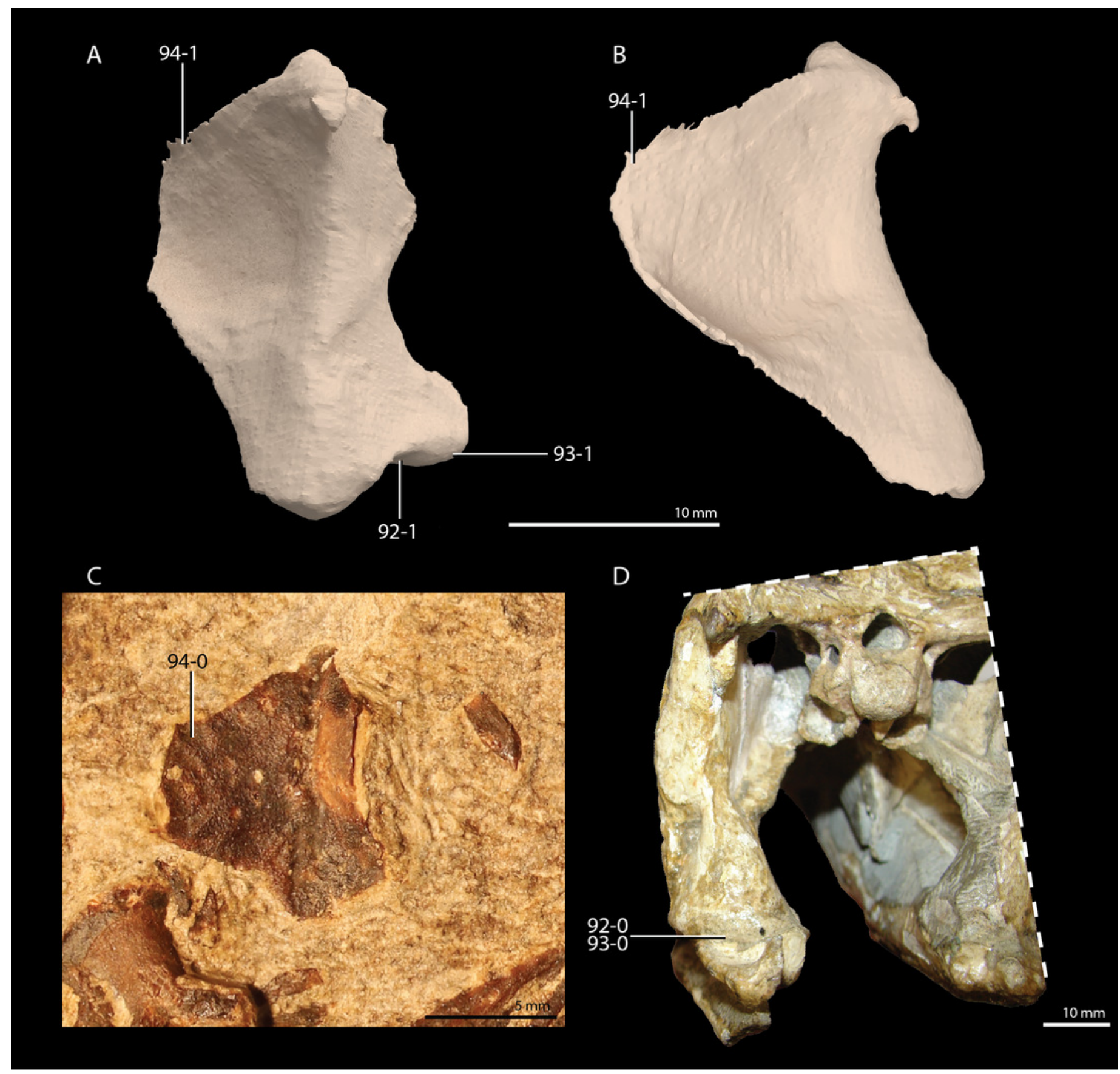




\section{Figure 16}

Illustration of characters 97, 101, 105, 108, 109, and 110.

(A) 105-0, 108-0, 109-0, and 110-1 in Macrocnemus fuyuanensis (palatal reconstruction, modified from Scheyer et al. 2020b). (B) 97-1, 101-1, 105-1, 108-0, 109-0, and 110-0 in Azendohsaurus madagaskarensis (palatal reconstruction, modified from Flynn et al. 2010). (C) 97-0, 101-1, 105-0, 108-0, and 109-1 in Tanystropheus longobardicus (palatal reconstruction, modified from Spiekman et al. 2020a). (D) 97-1, 108-1, 109-1, and 110-0 in Tanystropheus hydroides (palatal reconstruction, modified from Spiekman et al. 2020a).

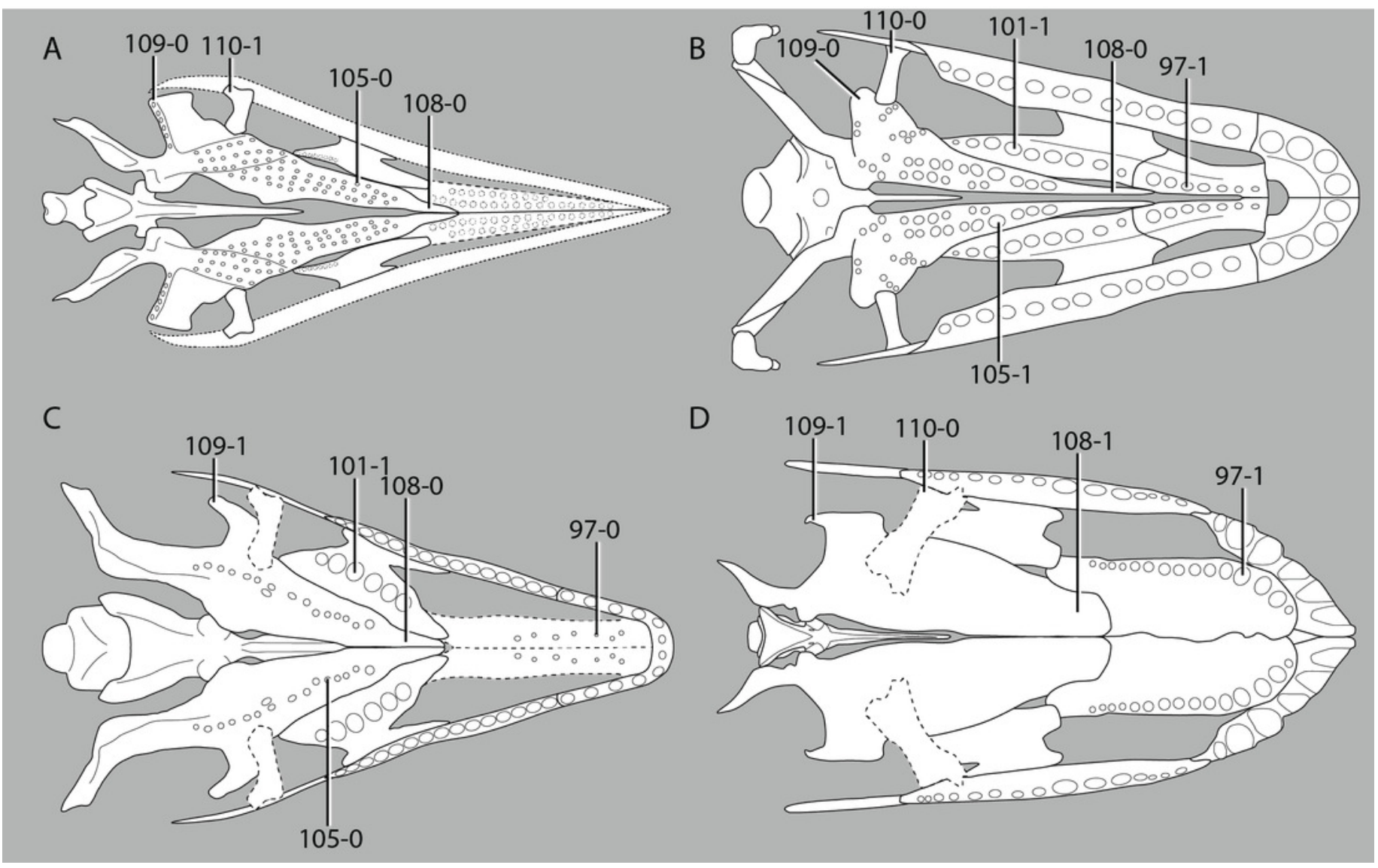




\section{Figure 17}

Illustration of characters 124 and 128.

(A) 124-0 and 128-1 in a digital reconstruction of Macrocnemus bassanii (PIMUZ T 2477, skull in posterior/occipital view). (B) 124-0 and 128-2 in Erythrosuchus africanus (BP/1/3893, partial braincase in posterior/occipital view). (C) 124-1 in a digital reconstruction of Tanystropheus hydroides (PIMUZ T 2790, skull in posterior view).

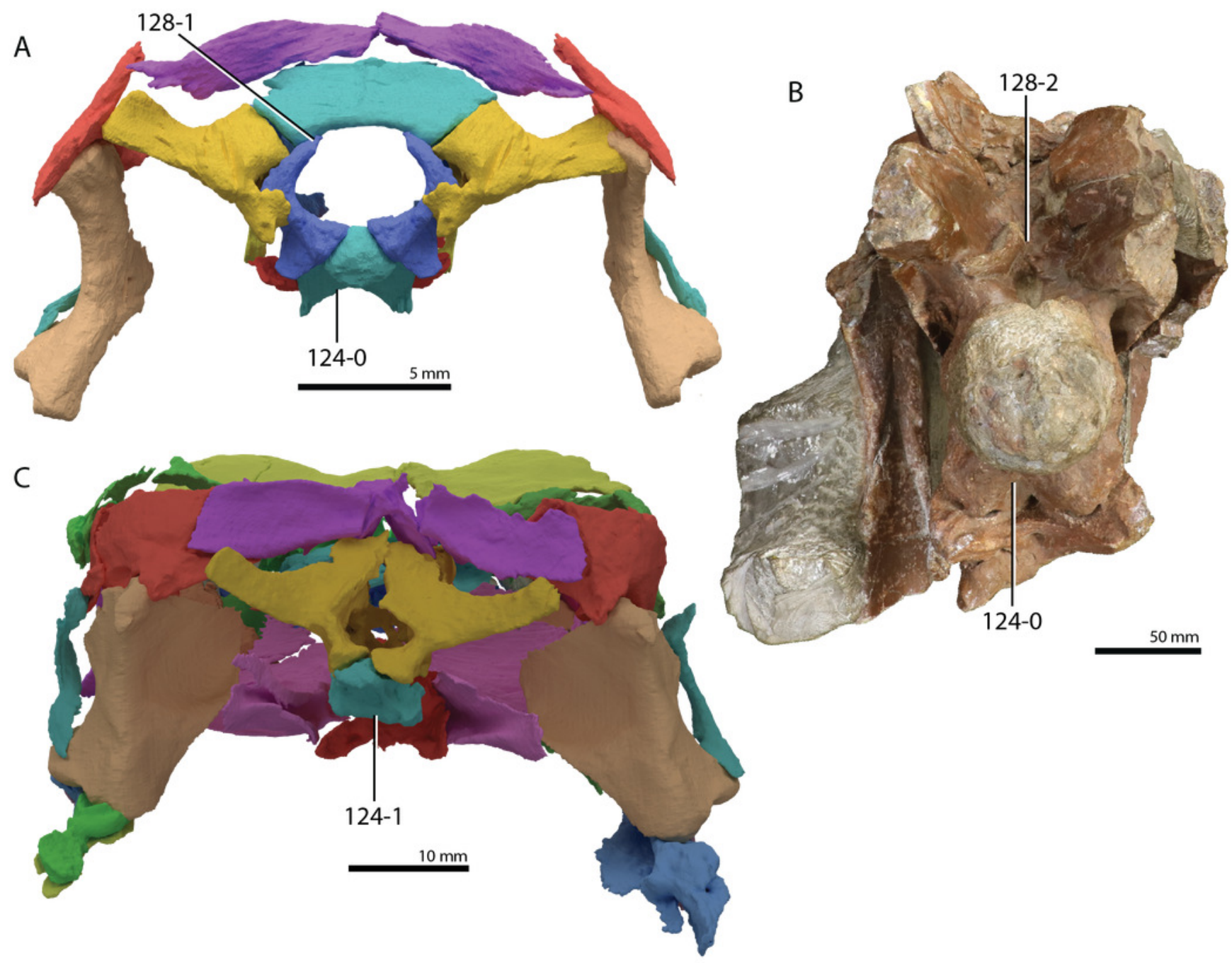




\section{Figure 18}

Illustration of characters $133,137,138,139$, and 140

(A) 133-1 in Prolacerta broomi (BP/1/2675, partial braincase in angled right posterolateroventral view). (B) 133-3, 137-1, 138-1, 139-1, 140-2 in Erythrosuchus africanus (BP/1/3893, partial braincase in angled right posterolateral view). (C) 133-2, 137-0, 138-1, 139-0, 140-0 in a digital reconstruction of Macrocnemus bassanii (PIMUZ T 2477, braincase right lateral view). (D) 133-2, 137-0, 138-1, 139-1, 140-1 in a digital reconstruction of Tanystropheus hydroides (PIMUZ T 2790, braincase right lateral view).

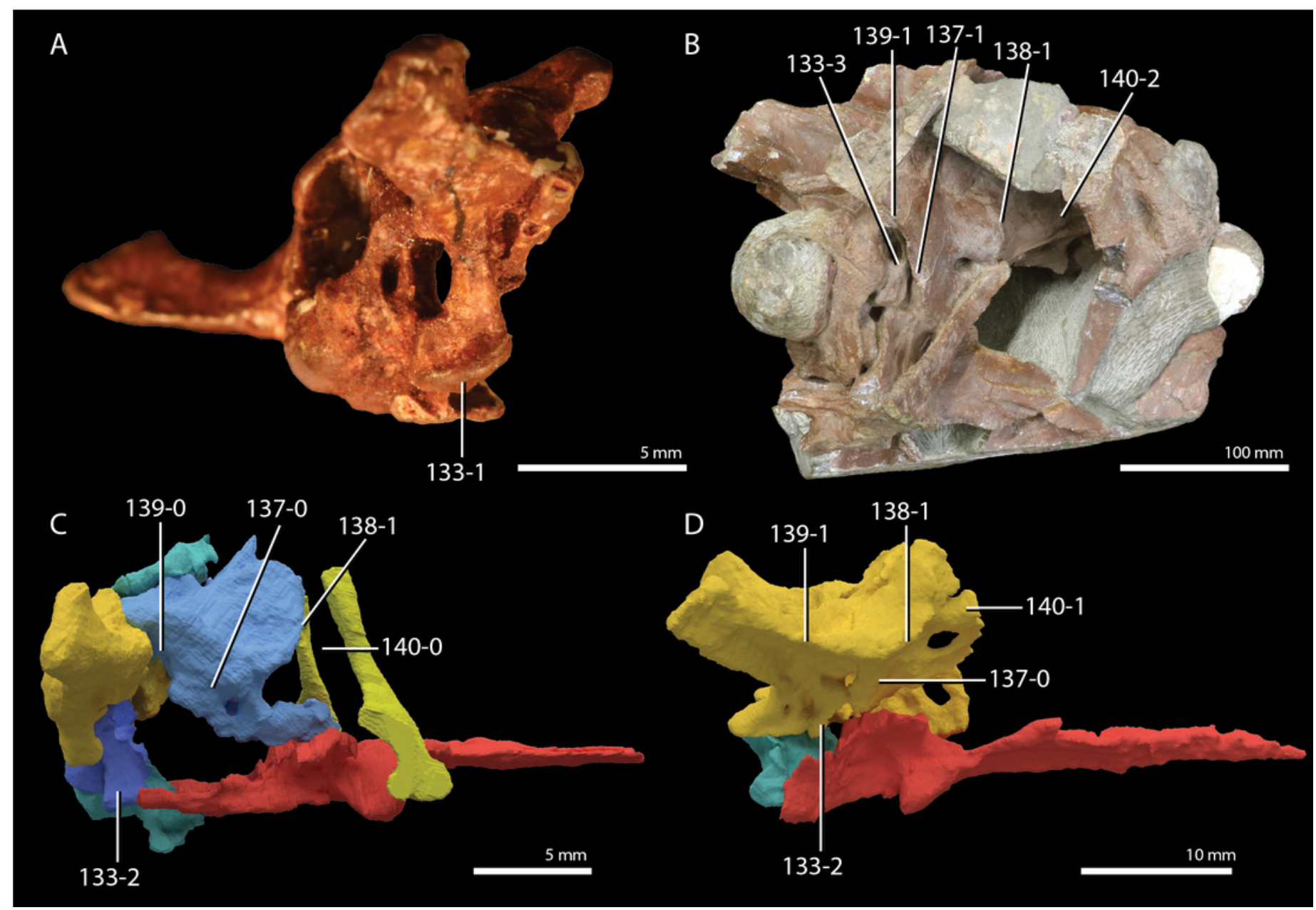




\section{Figure 19}

Illustration of characters 146, 158, 159, 161, and 165.

(A) 146-0, 158-0, 159-2, 161-0, and 165-0 in a digital reconstruction of Macrocnemus

bassanii (PIMUZ T 2477, right lower jaw in lateral view). (B) 146-1, 158-1, 159-1, 161-1, and 165-1 in a digital reconstruction of Tanystropheus hydroides (PIMUZ T 2790, left lower jaw in lateral view).

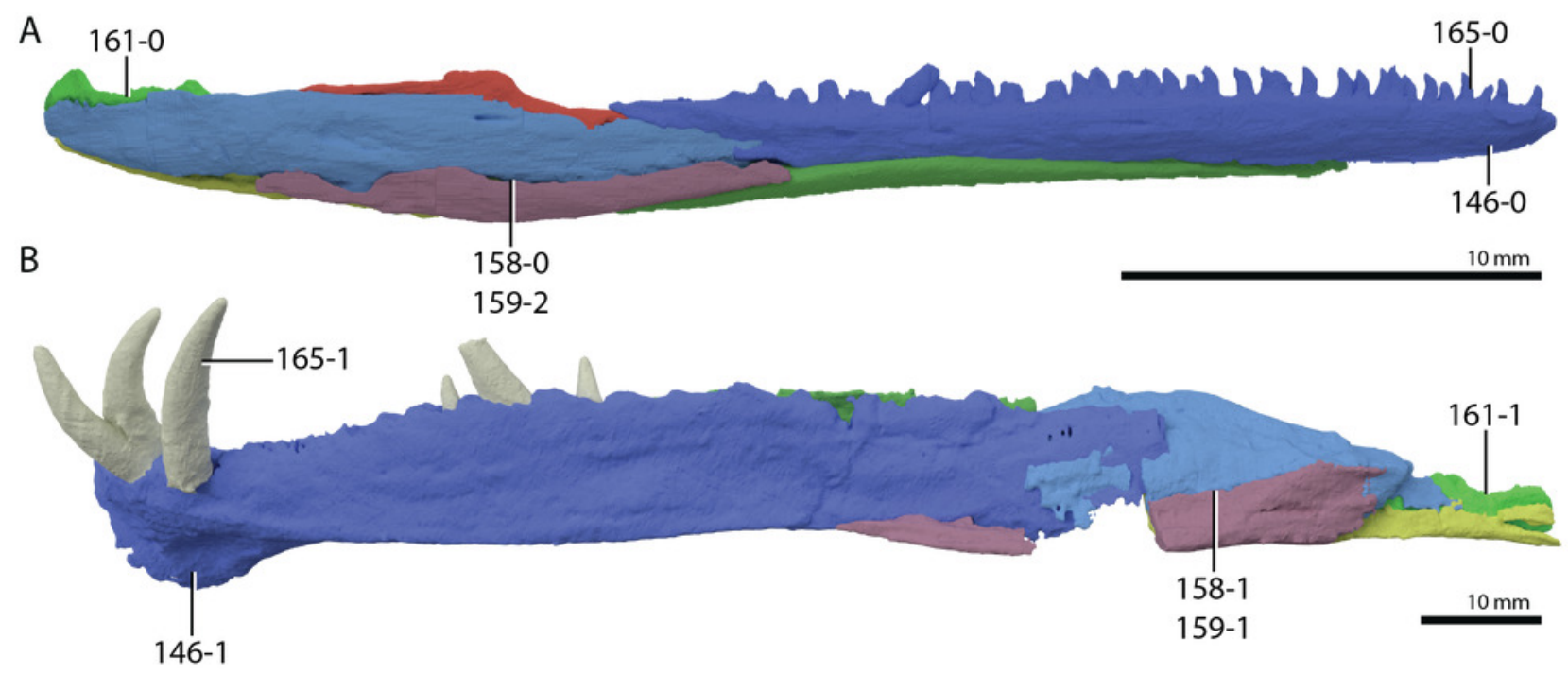




\section{Figure 20}

Illustration of character 174.

(A) State 1 in a digital reconstruction of Tanystropheus hydroides (PIMUZ T 2790, part of left maxilla in ventral view). (B) State 1 in a digital reconstruction of Macrocnemus bassanii (PIMUZ T 2477, part of left maxilla in ventral view; the colour of the teeth has been slightly modified in Adobe Illustrator CS6 to distinguish them from the maxilla).

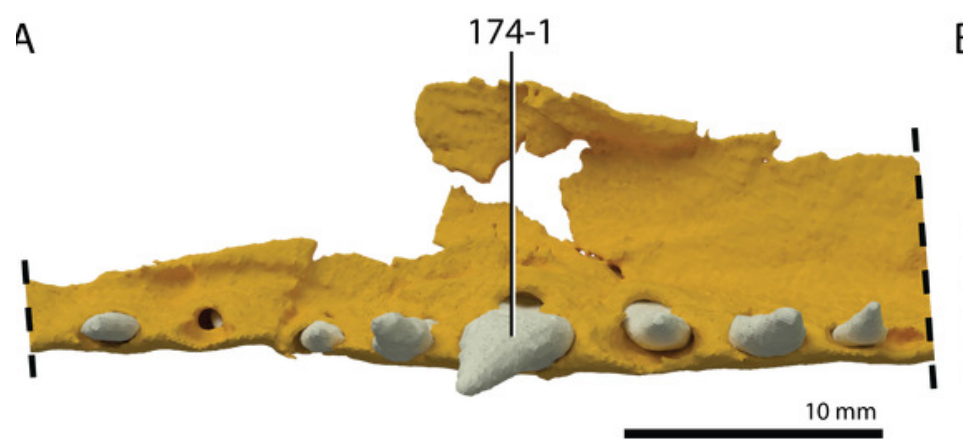

B

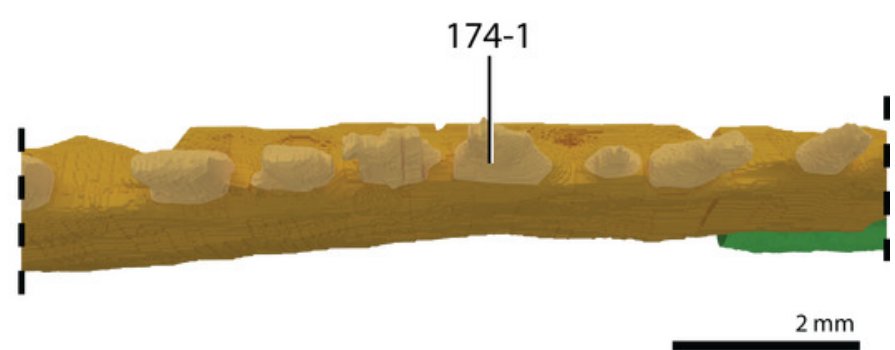




\section{Figure 21}

Illustration of character 178.

(A) State 4 in Sclerostropheus fossai (MCSNB 4035, mid-cervical vertebra in right lateral view). (B) State 3 in Tanystropheus hydroides (PIMUZ T 2819, mid-cervical vertebrae in left lateral view). (C) State 2 in Fuyuansaurus acutirostris (IVPP V17983, mid-cervical vertebrae in left lateral view). (D) State 0 in Youngina capensis (BP/1/3859, anterior cervical vertebrae right lateral view).

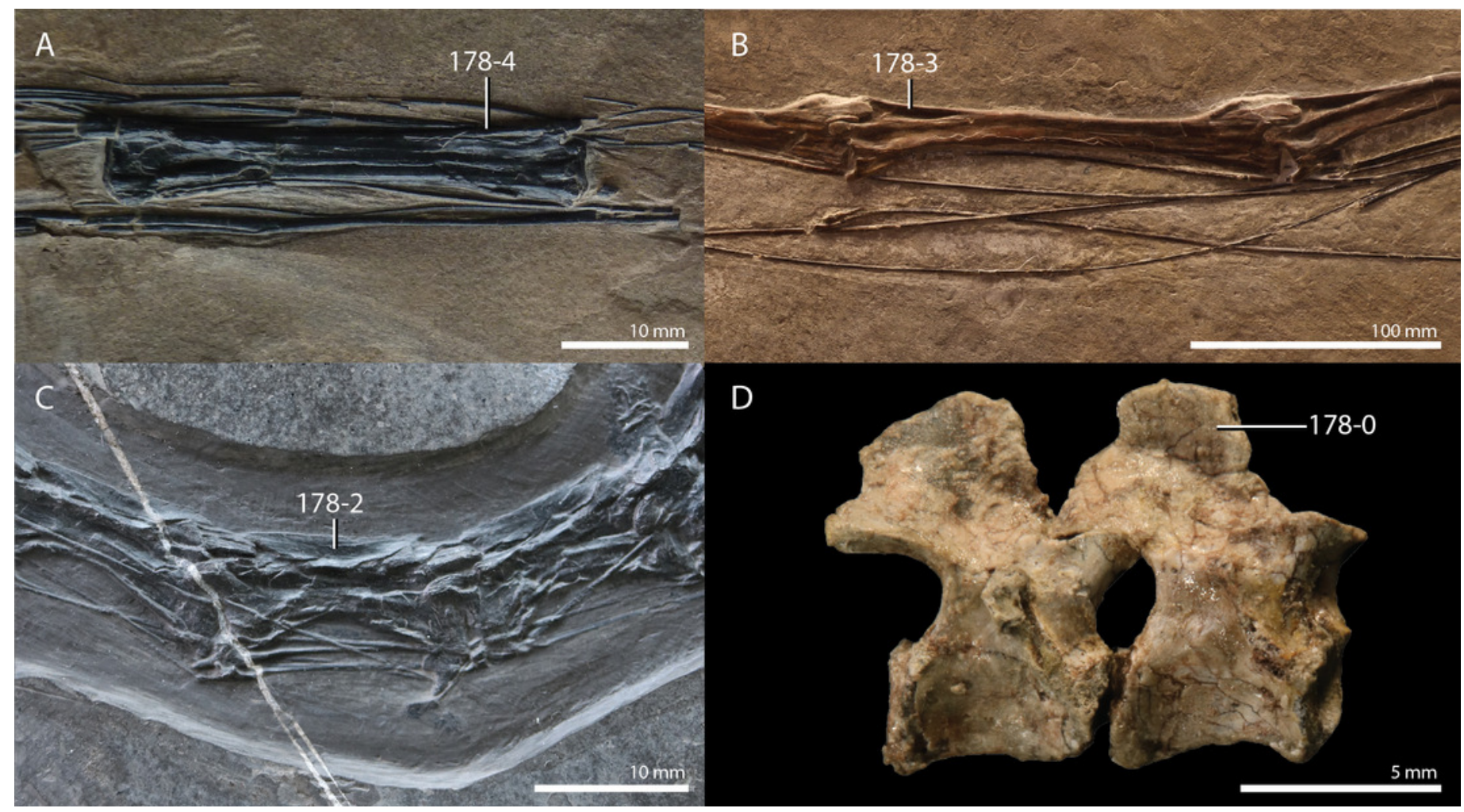




\section{Figure 22}

Illustration of characters 179 and 180.

(A) 179-1 and 180-2 in Macrocnemus fuyuanensis (IVPP V15001, anterior cervical vertebrae in right lateral view). (B) 179-0 and 180-0 in Mesosuchus browni (SAM-PK-5882, anterior cervical vertebrae in left lateral view).

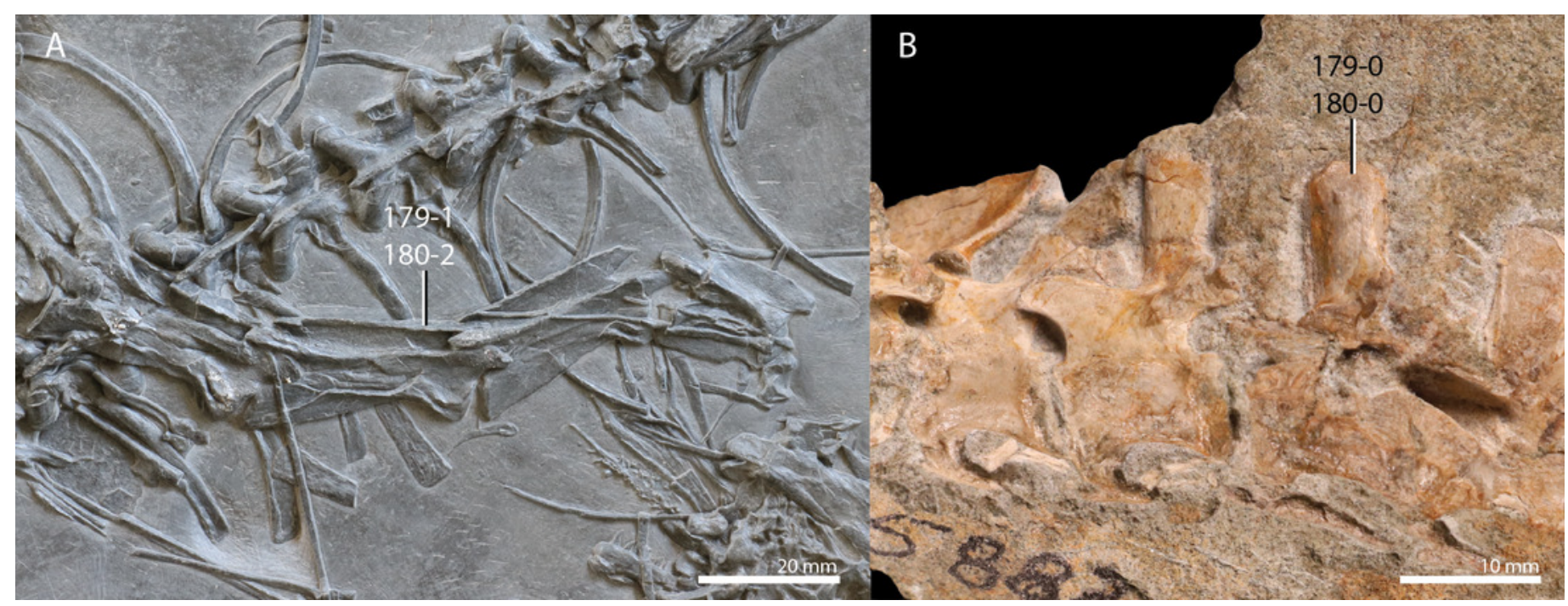




\section{Figure 23}

Illustration of character 184.

(A) State 1 in a digital reconstruction of Tanystropheus hydroides (PIMUZ T 2790, atlas-axis complex in right lateral view). (B) State 0 in a digital reconstruction of Macrocnemus bassanii (PIMUZ T 2477, atlas-axis complex in left lateral view).

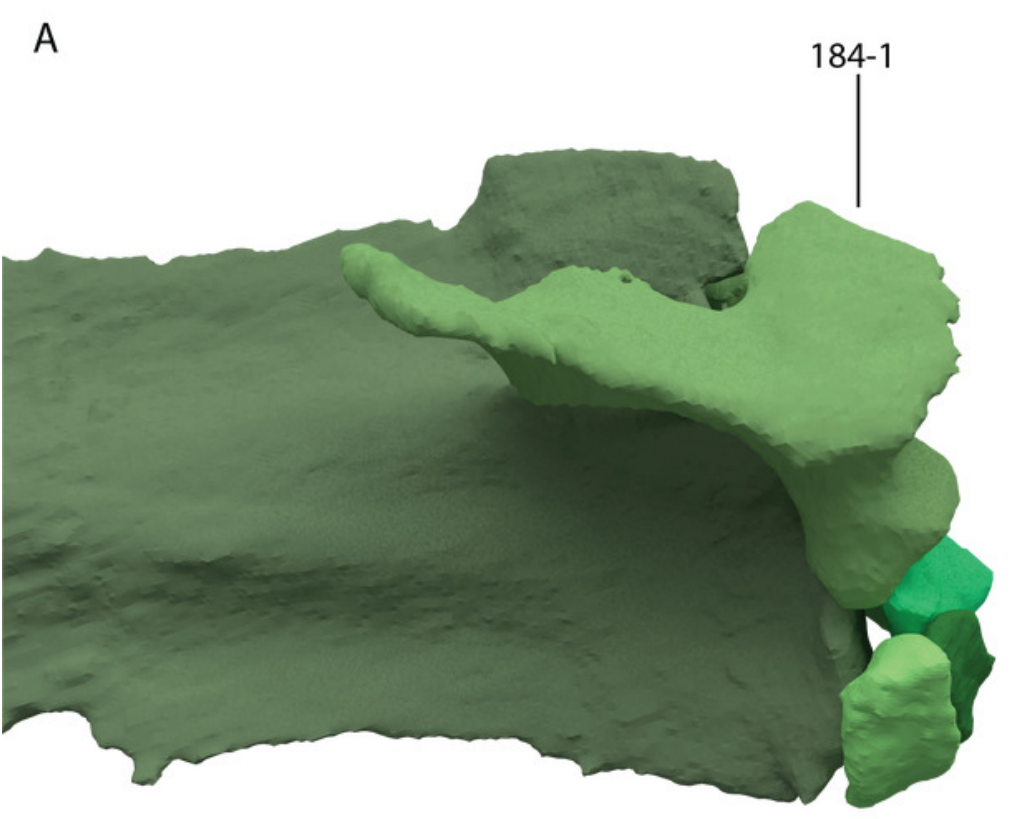

$10 \mathrm{~mm}$
B

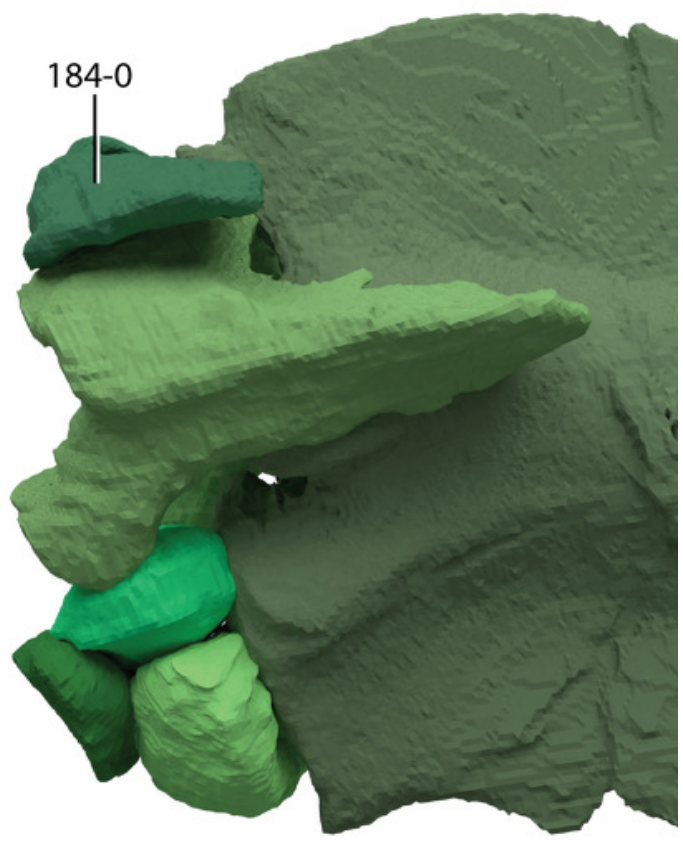




\section{Figure 24}

Illustration of character 191.

(A) State 0 in Protorosaurus speneri (WMsN P 47361, posterior cervical vertebrae in right lateral view). (B) State 1 in Tanystropheus "conspicuus" (U-MO BT 733, posterior part of midcervical vertebra in right lateral view).

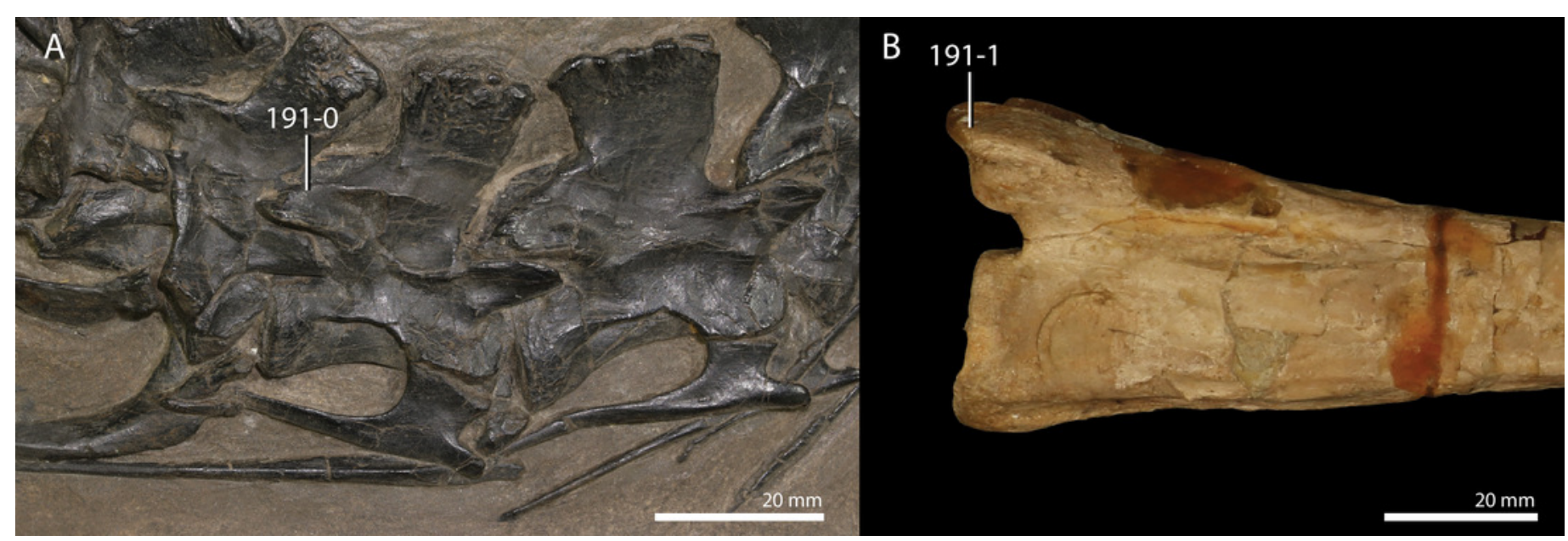




\section{Figure 25}

Illustration of character 192.

(A) State 0 in Pamelaria dolichotrachela (ISIR 316, anterior cervical vertebra in dorsal view).

(B) State 1 in Tanystropheus "conspicuus" (U-MO BT 740, posterior part of mid-cervical vertebra in dorsal view). Image of Pamelaria dolichotrachela courtesy of Martín Ezcurra.

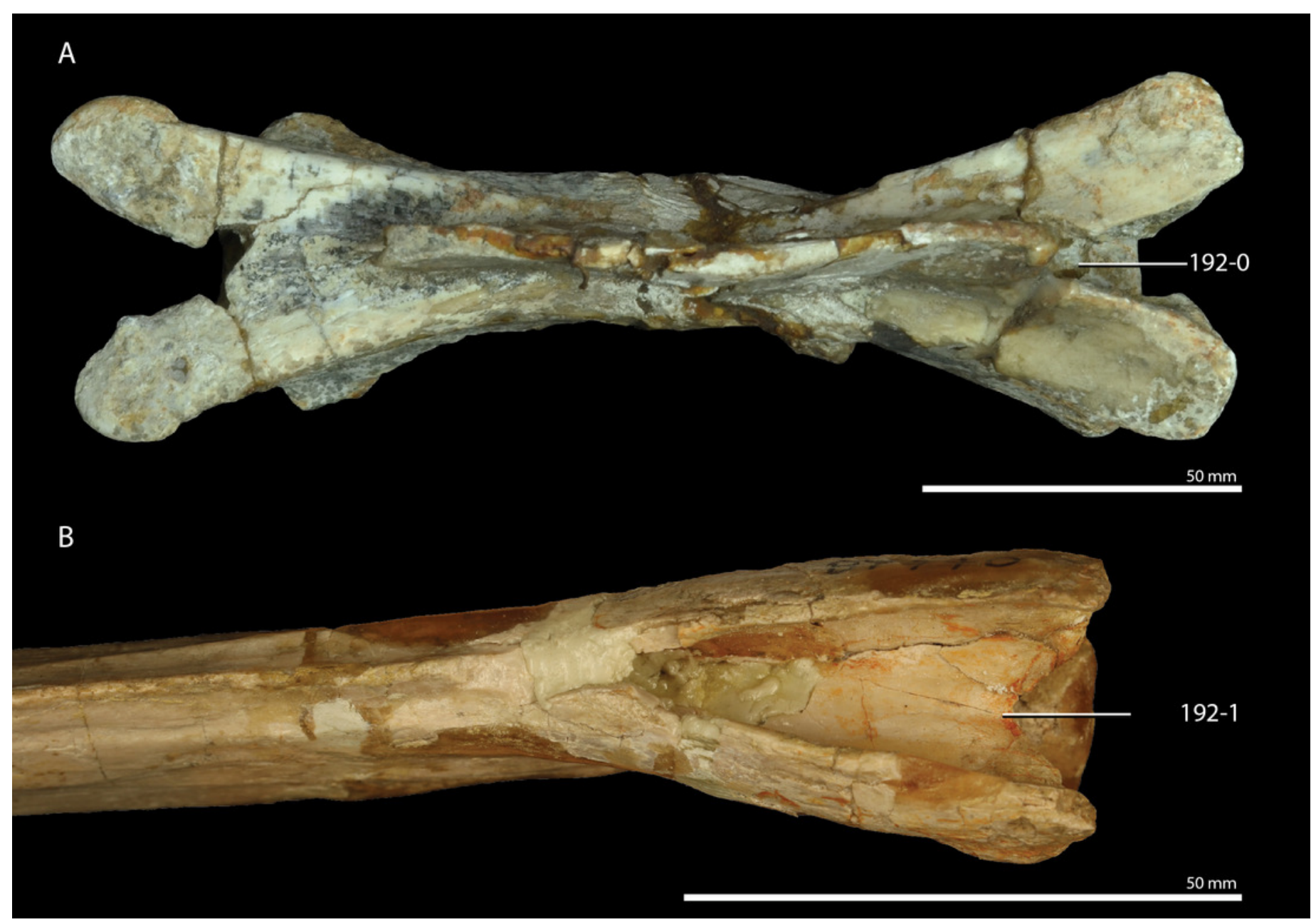




\section{Figure 26}

Illustration of character 196.

(A) State 0 in Youngina capensis (BP/1/3859, anterior cervical vertebra in ventral view). (B) State 1 in Tanystropheus "conspicuus" (U-MO BT 733, partial cervical vertebra in ventral view).

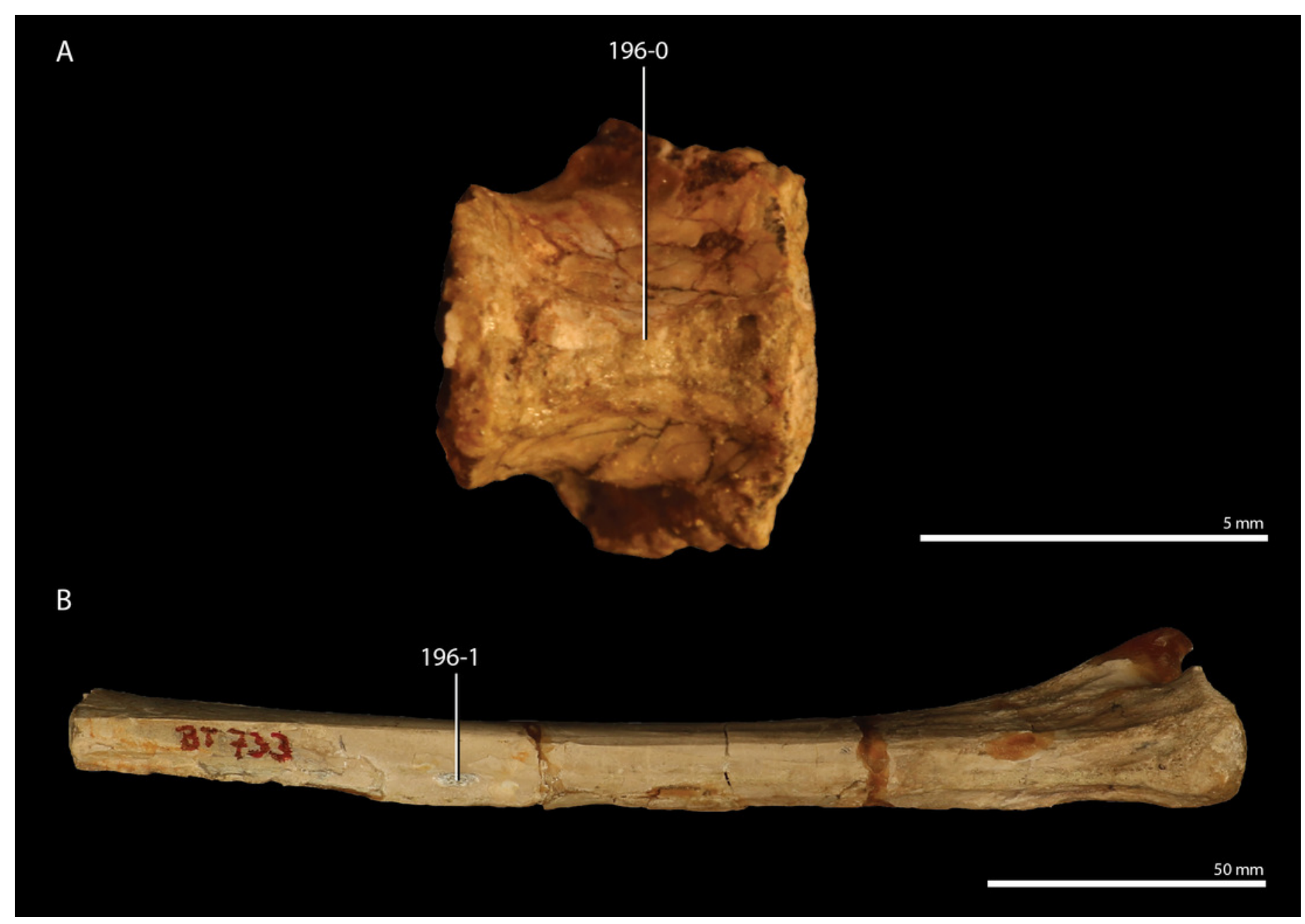




\section{Figure 27}

Illustration of characters 199 and 200.

(A) 199-2 and 200-1 in Tanystropheus longobardicus (PIMUZ T 3901, skull and partial cervical column in left lateral view). (B) 199-1 and 200-2 in Tanytrachelos ahynis (VMNH 120346a, partial skeleton including cervical column, cervical column in left lateral view). (C) 199-2 and 200-2 in Pectodens zhenyuensis (IVPP V18578, skull and cervical column in right lateral view). 


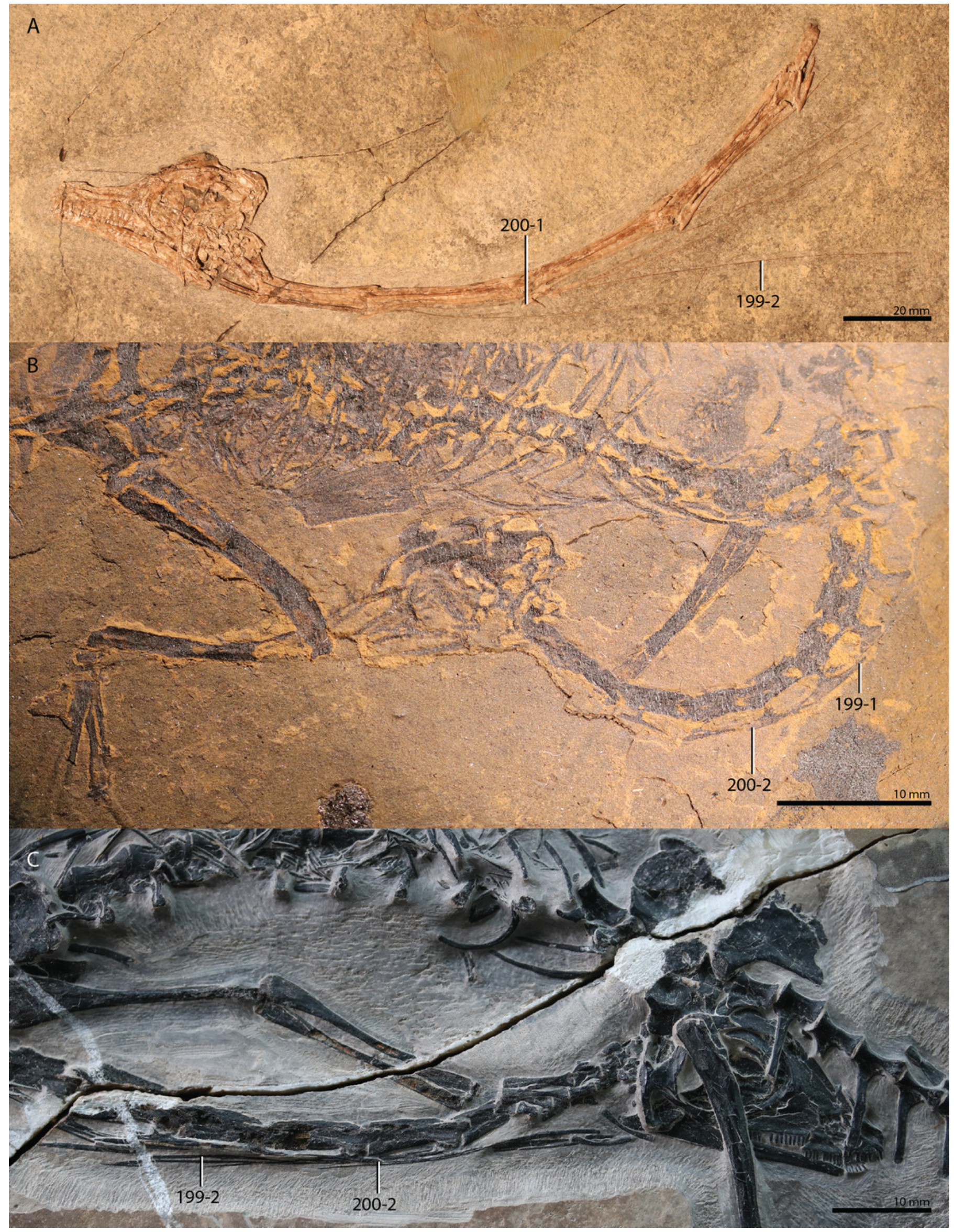




\section{Figure 28}

Illustration of characters 202 and 203.

(A) 202-1 and 203-2 in Tanystropheus longobardicus (PIMUZ T 1277, disarticulated anterior dorsal vertebrae, indicated vertebra in angled left dorsolateral view). (B) 202-0 and 203-1 in Euparkeria capensis (SAM-PK-6047A, dorsal vertebral column in right lateral view). (C) 202-0 and 203-0 in Mesosuchus browni (SAM-PK-6046, dorsal vertebral column in left lateral view).

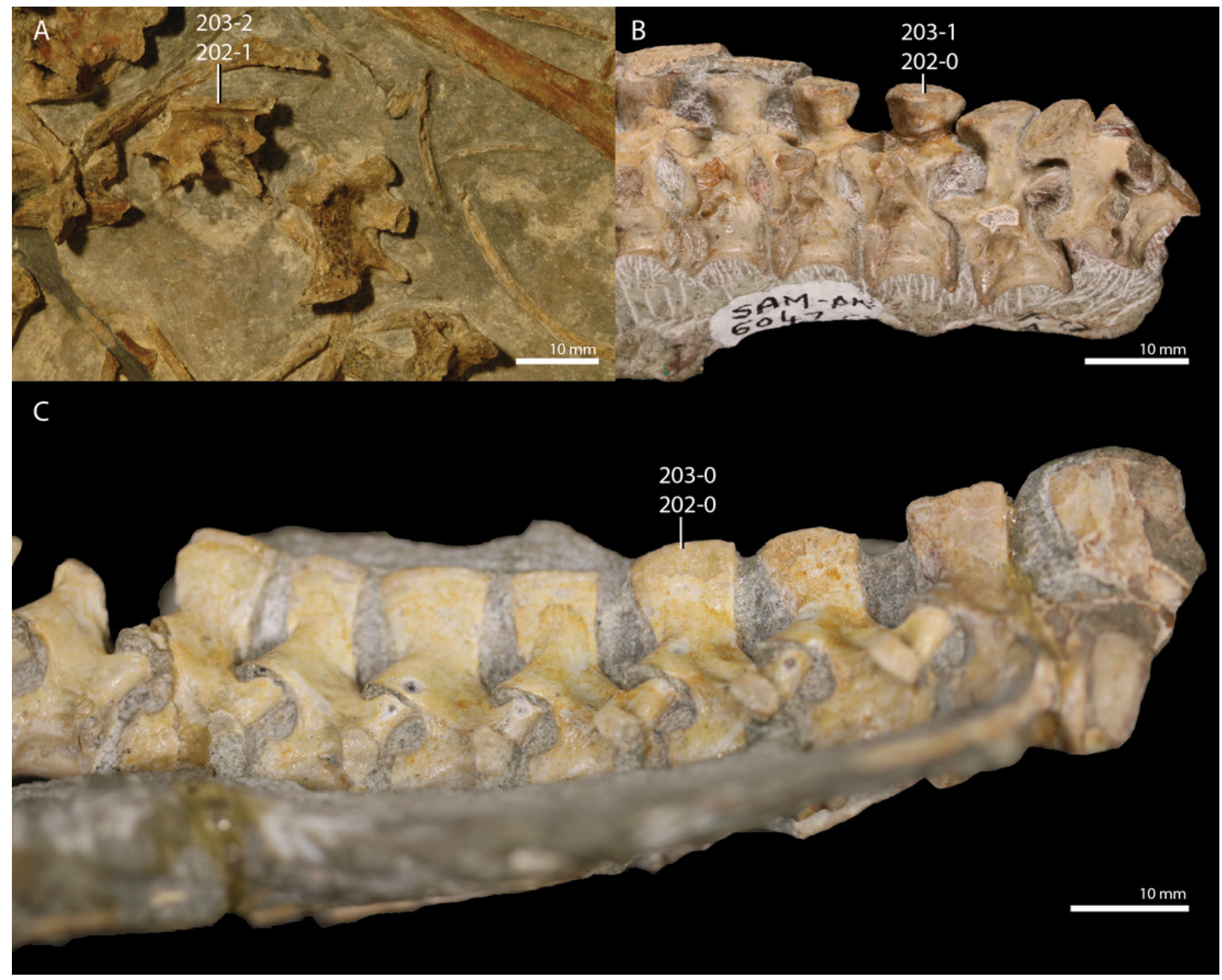




\section{Figure 29}

Illustration of character 224

(A) State 0 in Protorosaurus speneri (WMsN P 47361, largely complete skeleton in right lateral view, scale bar in cm). (B) State 1 in Pectodens zhenyuensis (IVPP V18578, largely complete skeleton, largely in ventral view).

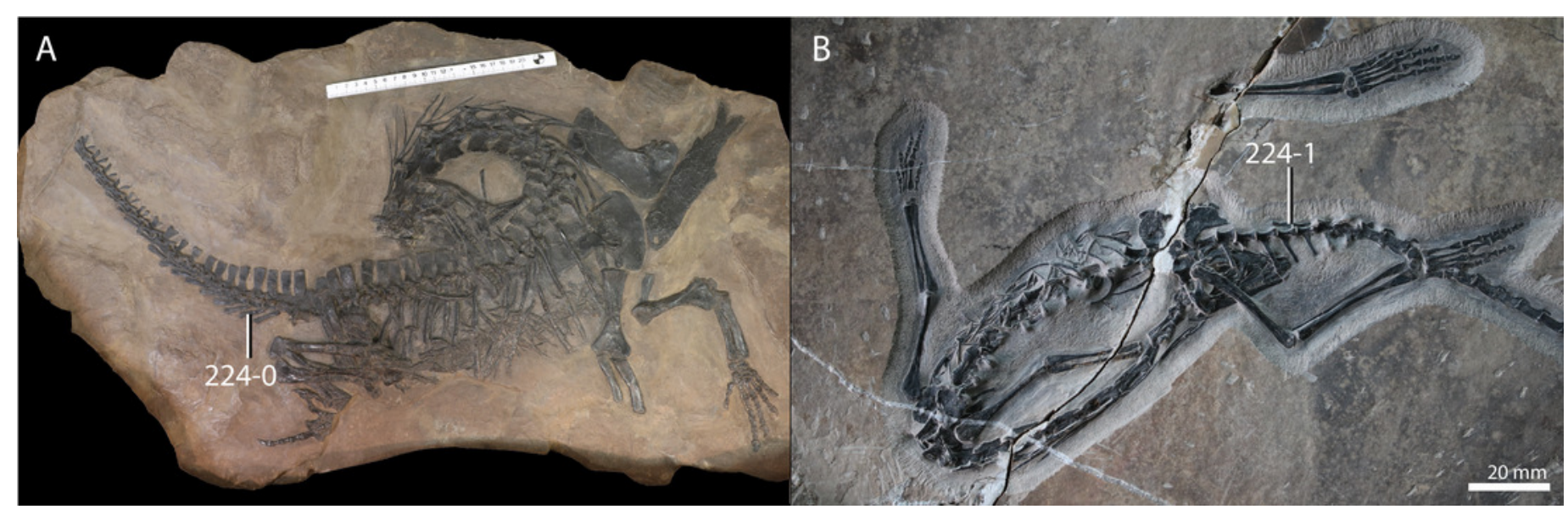




\section{Figure 30}

Illustration of character 225 state 1 in Tanytrachelos ahynis (VMNH 120013, largely complete skeleton exhibiting heterotopic bones).

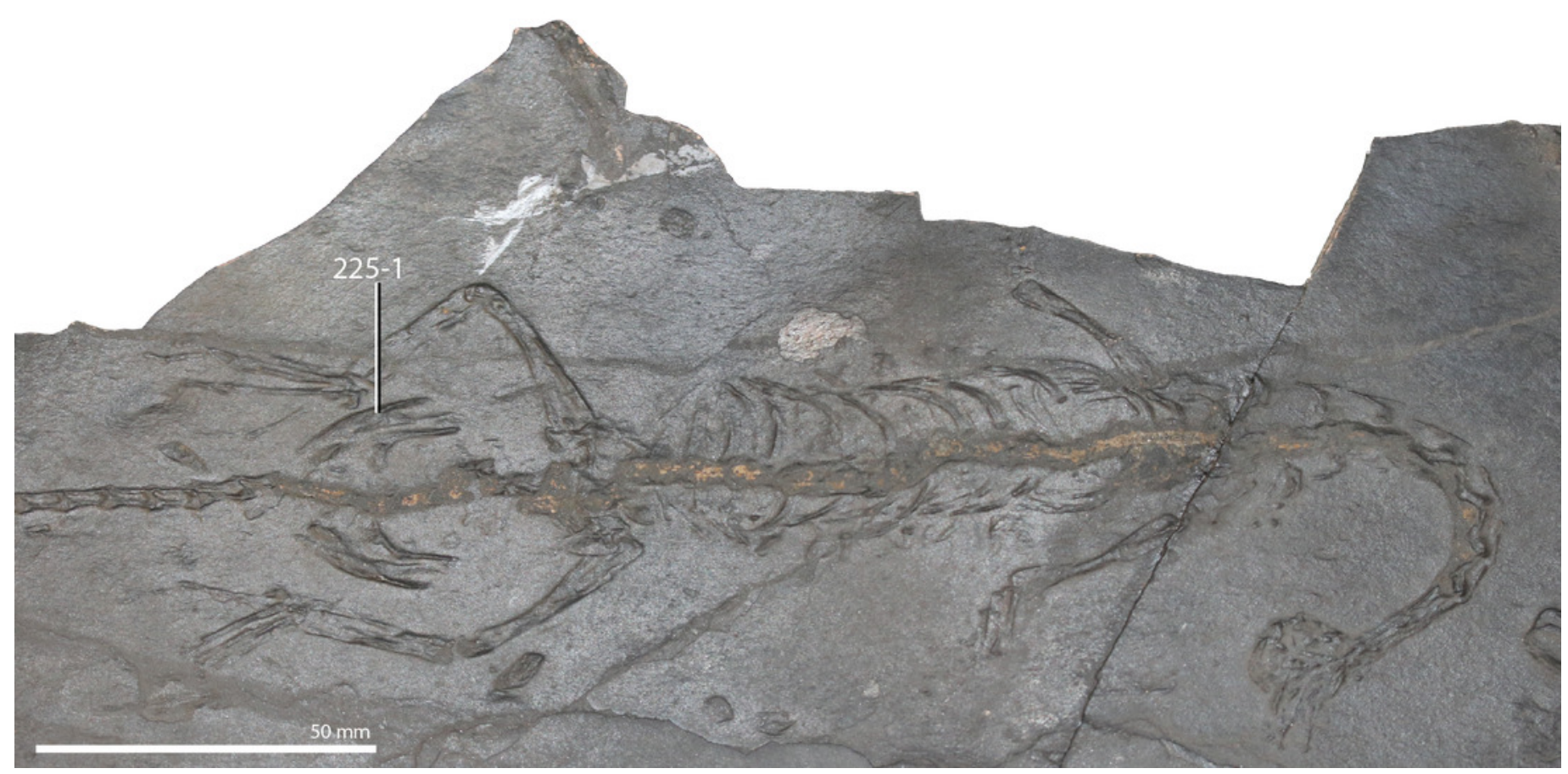


Figure 31

Illustration of characters 227 and 228.

(A) 227-2 and 228-1 in Tanystropheus longobardicus (PIMUZ T 1277, left scapulocoracoid in medial view). (B) 227-0 and 228-0 in Protorosaurus speneri (WMsN P 47361, right scapulocoracoid in lateral view). (C) 227-1 and 228-0 in Euparkeria capensis (SAM-PK-5867, partial articulated skeleton including pectoral girdle in angled right lateroventral view). 


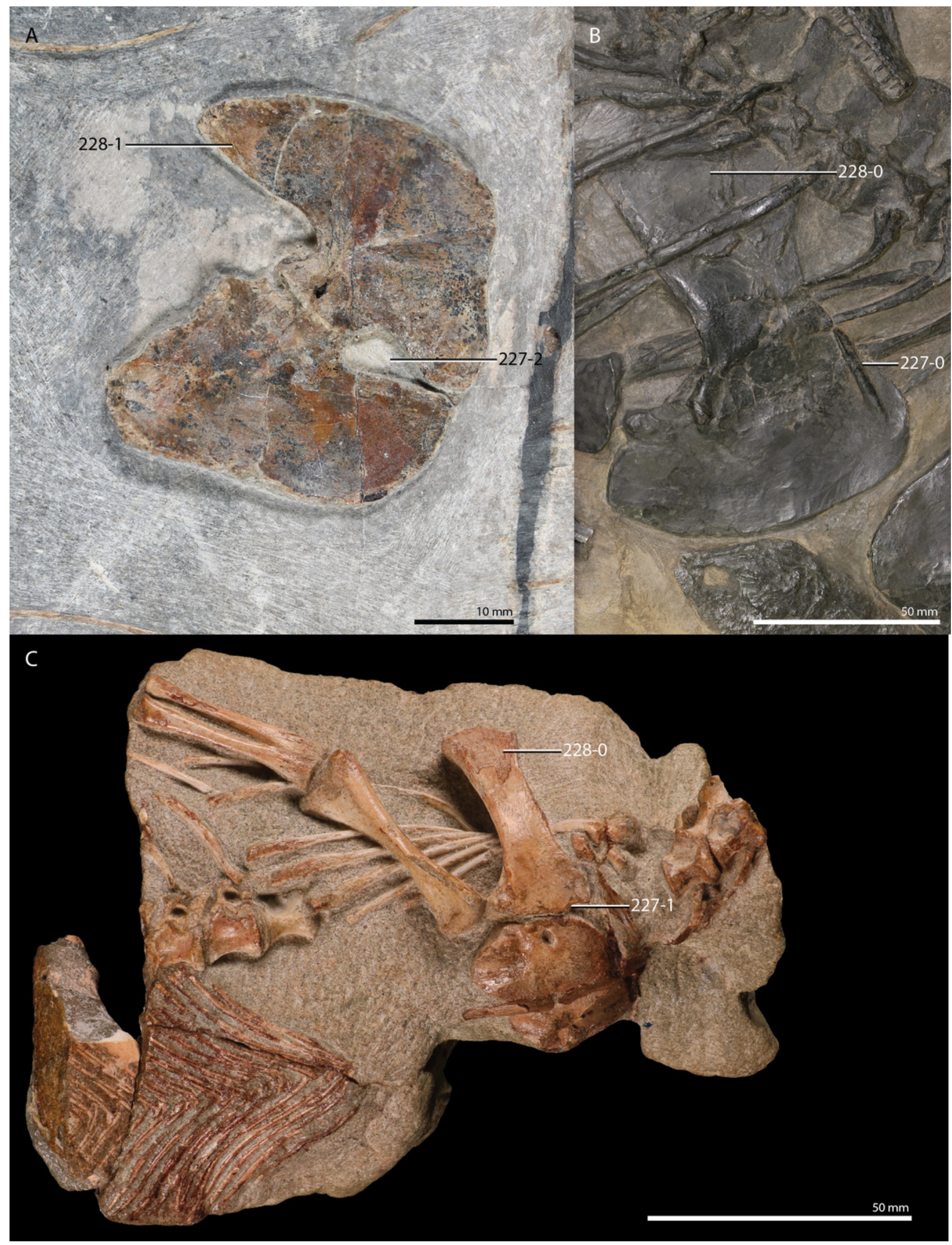




\section{Figure 32}

Illustration of characters 266 and 278.

(A) 266-0 and 278-1 in Macrocnemus fuyuanensis (IVPP V15001, right half of the pelvic girdle in medial view). (B) 266-1 in Tanystropheus longobardicus (PIMUZ T 1277, right ilium in medial view). (C) 278-0 Tanystropheus hydroides (PIMUZ T 2817, ischia in lateral view).

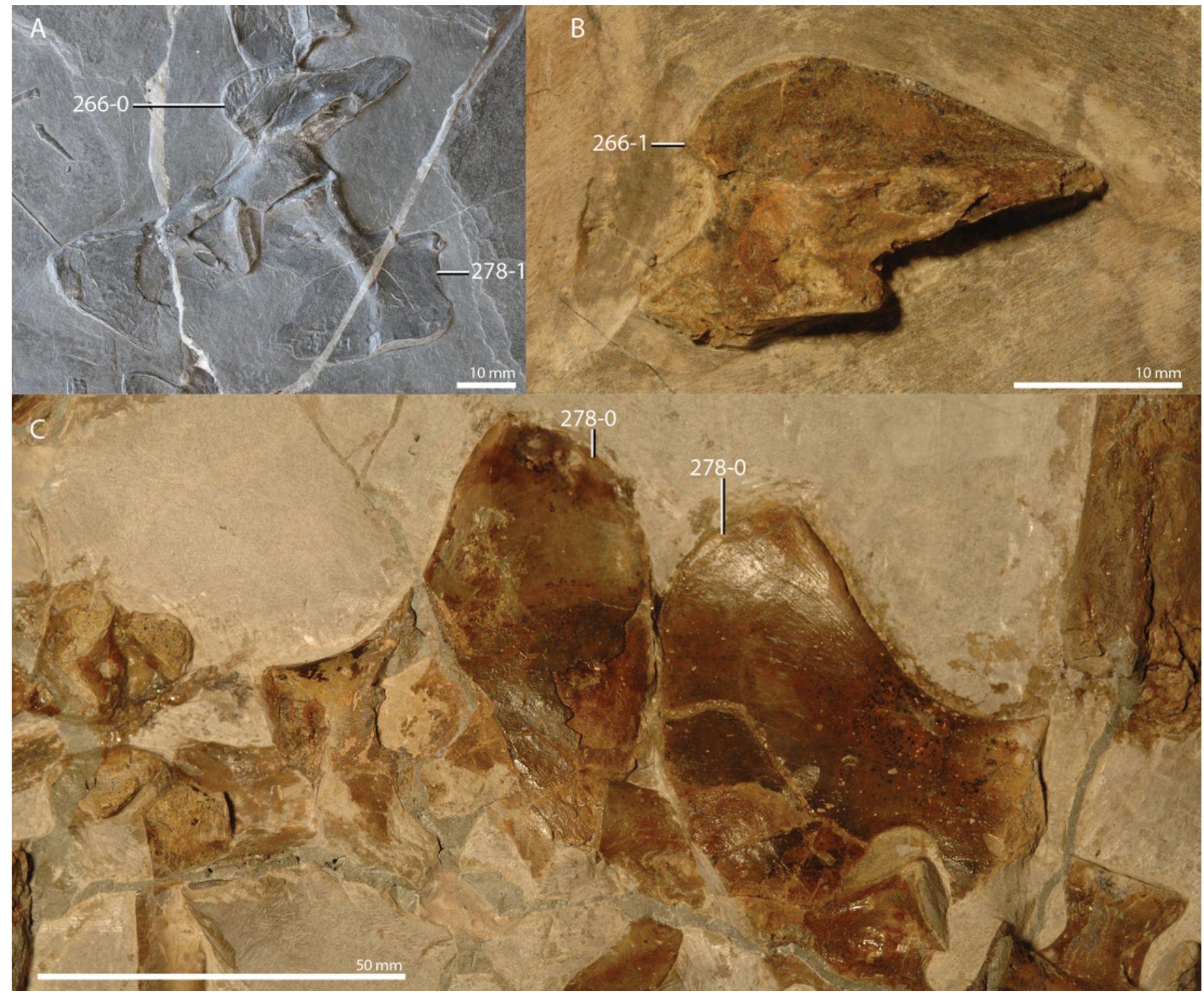




\section{Figure 33}

Results of analysis 1 (ratio characters excluded and all characters treated as unordered; all OTUs included).

(A) Strict consensus tree out of 1976 MPTs with 977 steps. Bremer values above 1 and Bootstrap frequencies above $50 \%$ are provided above and below each node, respectively. (B) First reduced strict consensus tree after the a posteriori exclusion of Acerosodontosaurus piveteaui and Macrocnemus obristi. (C) Second reduced strict consensus tree after additionally excluding Elessaurus gondwanoccidens a posteriori. (D) Third reduced strict consensus tree after additionally excluding Pectodens zhenyuensis a posteriori. OTUs formerly considered to be "protorosaurs" are highlighted in bold. 
A

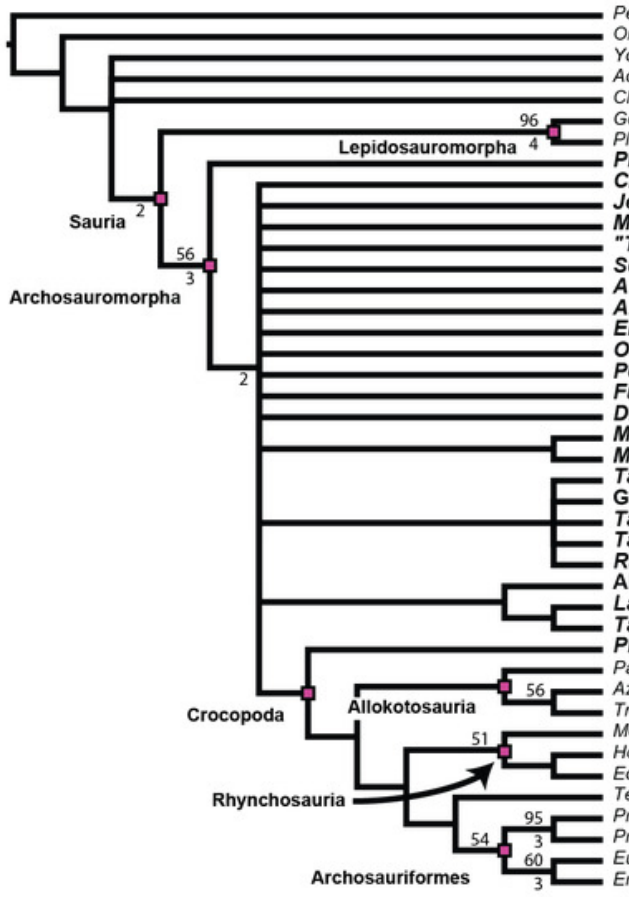

C

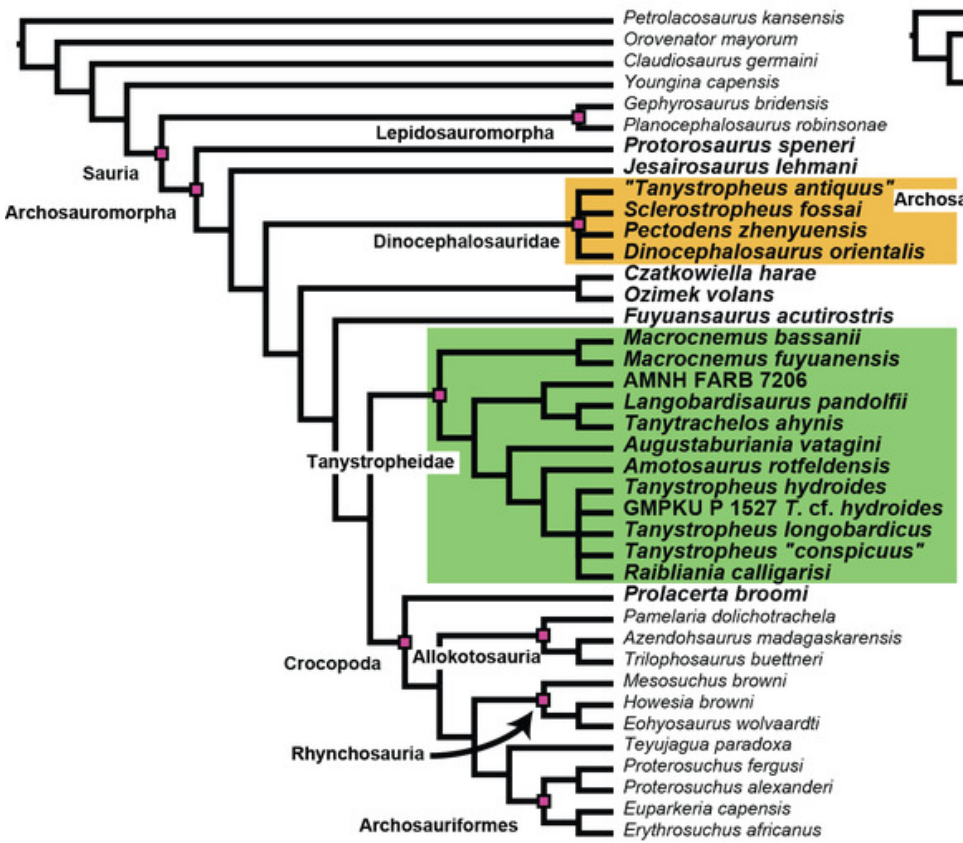

B

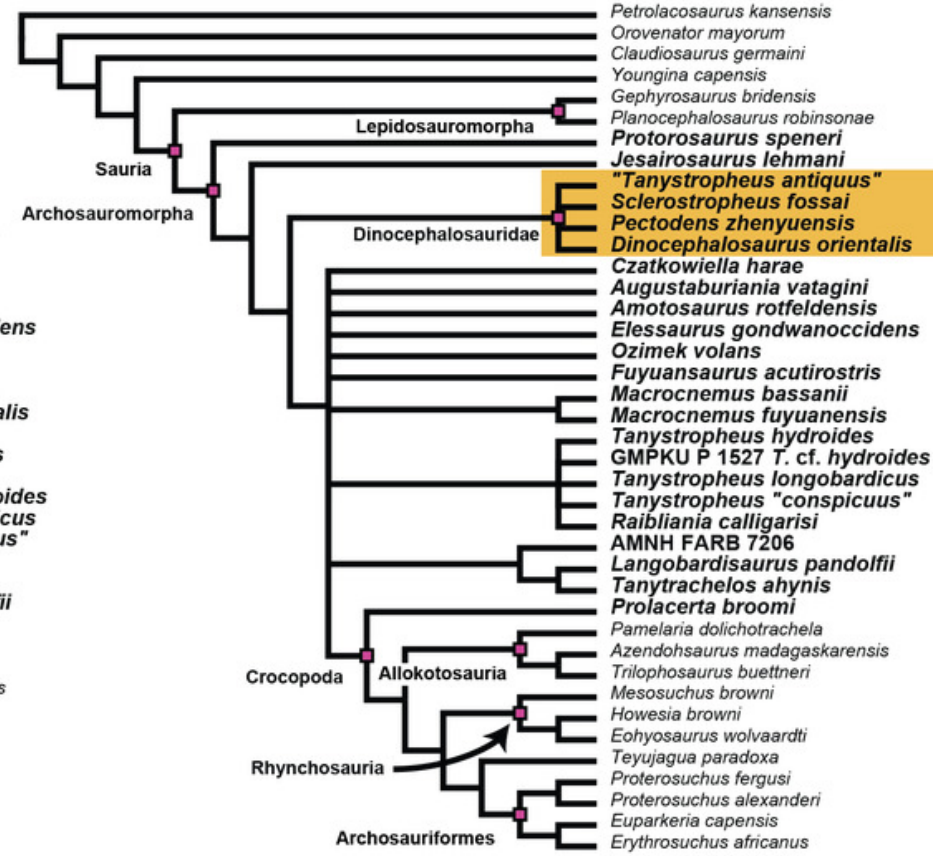

D

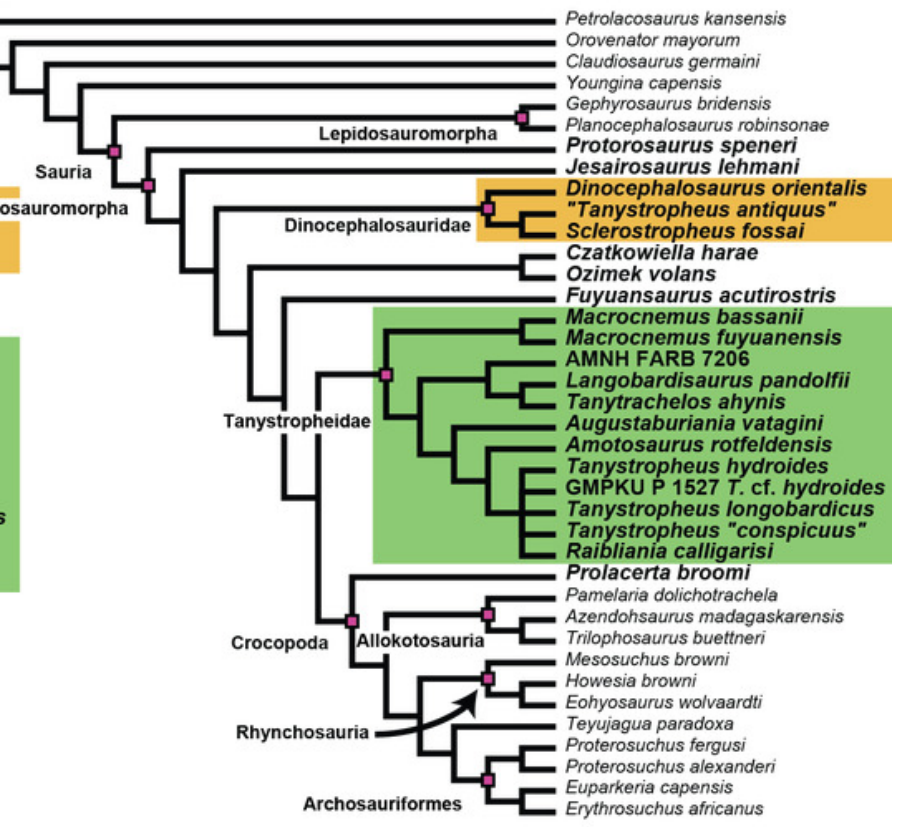




\section{Figure 34}

Results of analysis 2 (ratio characters excluded and all characters treated as unordered; Czatkowiella harae, Tanystropheus "conspicuus", and "Tanystropheus antiquus" pruned a priori).

(A) Strict consensus tree out of 884 MPTs with 953 steps. Bremer values above 1 and Bootstrap frequencies above $50 \%$ are provided above and below each node, respectively. (B) First reduced strict consensus tree after the a posteriori exclusion of Acerosodontosaurus piveteaui and Macrocnemus obristi. (C) Second reduced strict consensus tree after additionally excluding Sclerostropheus fossai, Elessaurus gondwanoccidens, Pectodens zhenyuensis, Fuyuansaurus acutirostris, and Dinocephalosaurus orientalis a posteriori. (D) Third reduced strict consensus tree after additionally excluding Jesairosaurus lehmani and GMPKU P 1527 a posteriori. OTUs formerly considered to be "protorosaurs" are highlighted in bold. 
A

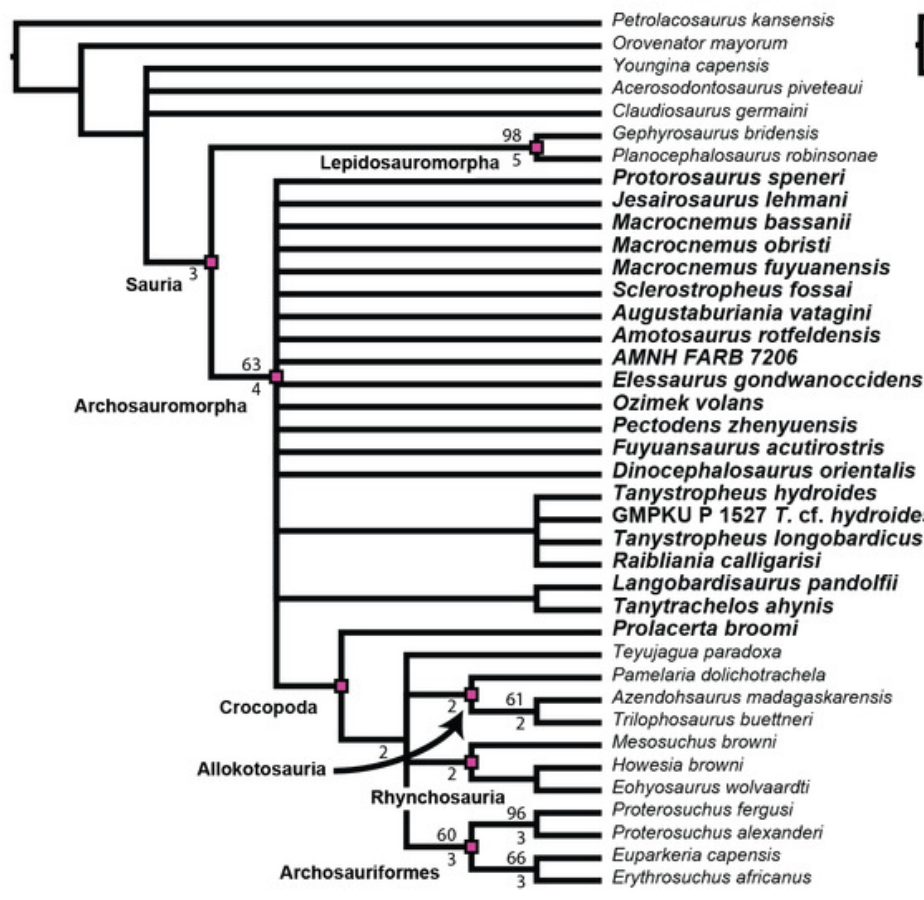

C

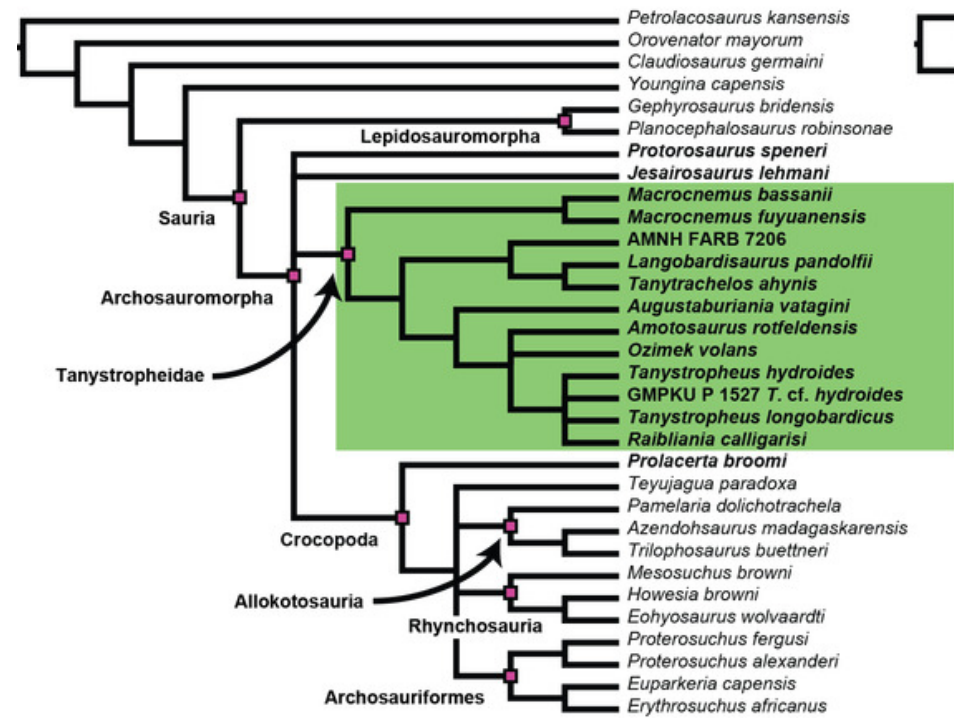

B

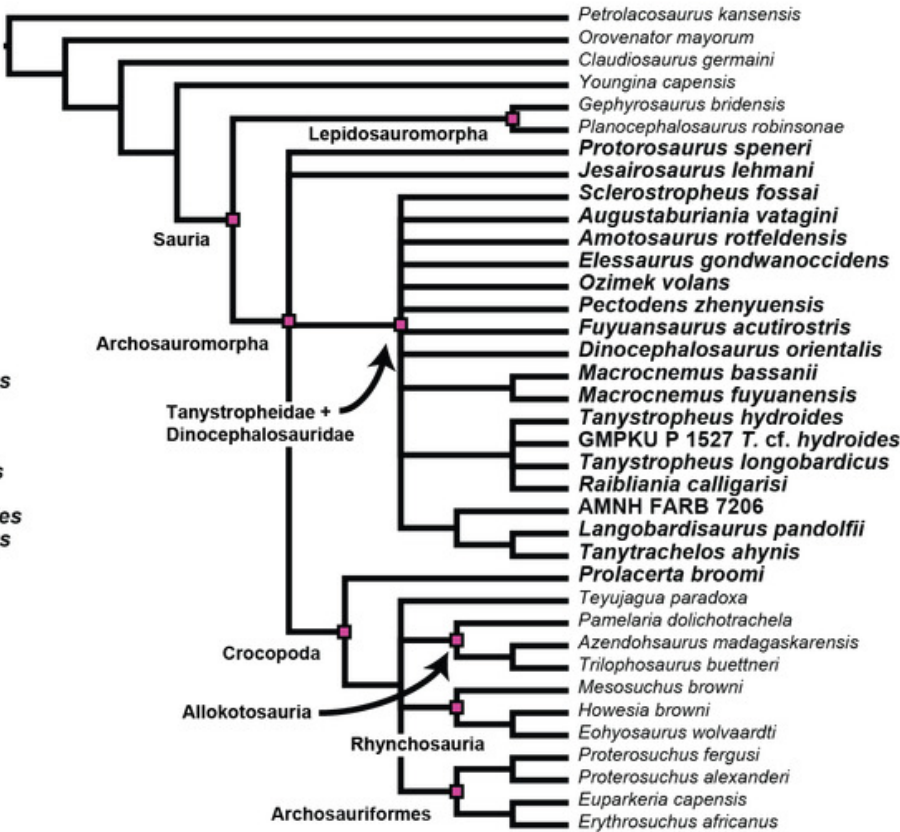

D

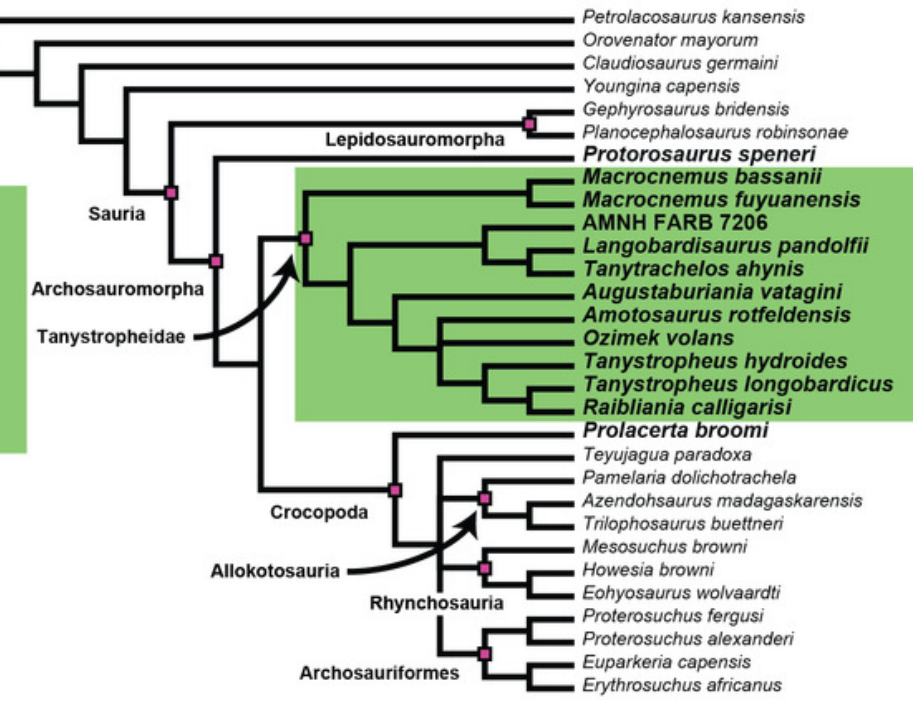




\section{Figure 35}

Results of analysis 3 (ratio characters included and specified characters ordered; all OTUs included).

(A) Strict consensus tree out of 434 MPTs with 1270 steps. Bremer values above 1 and Bootstrap frequencies above $50 \%$ are provided above and below each node, respectively. (B) First reduced strict consensus tree after the a posteriori exclusion of Macrocnemus obristi and Elessaurus gondwanoccidens. (C) Second reduced strict consensus tree after additionally excluding Tanytrachelos ahynis a posteriori. (D) Third reduced strict consensus tree after additionally excluding Tanystropheus "conspicuus" and Raibliania calligarisi a posteriori. OTUs formerly considered to be "protorosaurs" are highlighted in bold. 
A

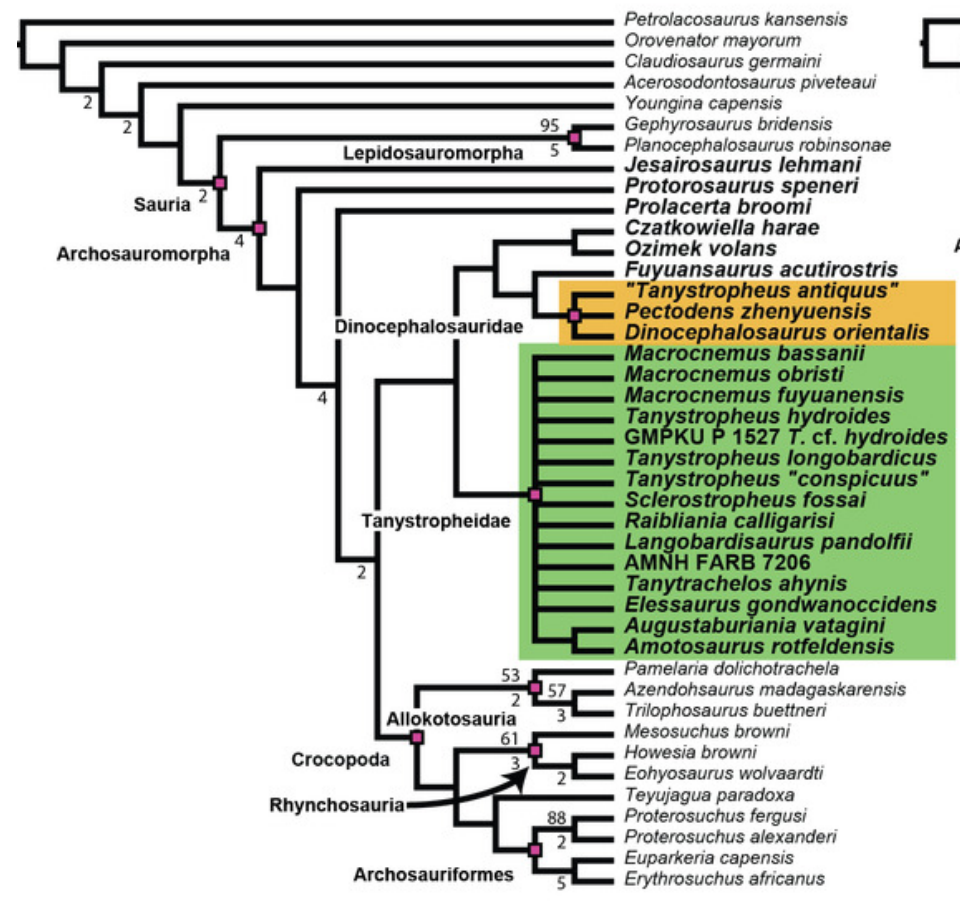

B

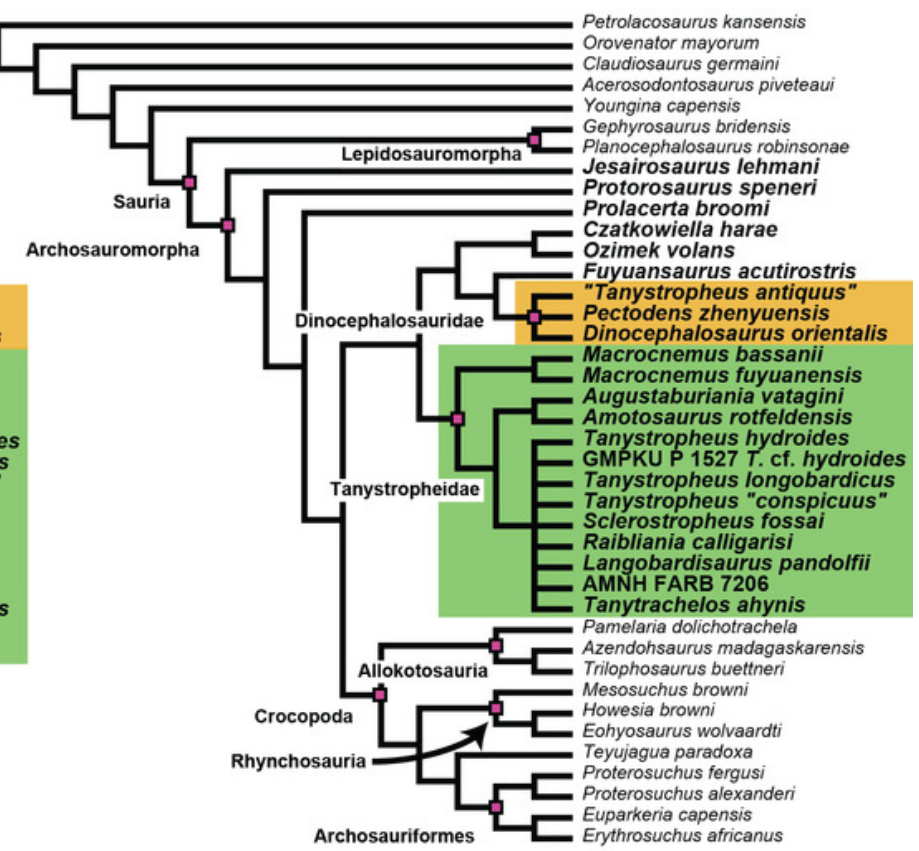

D

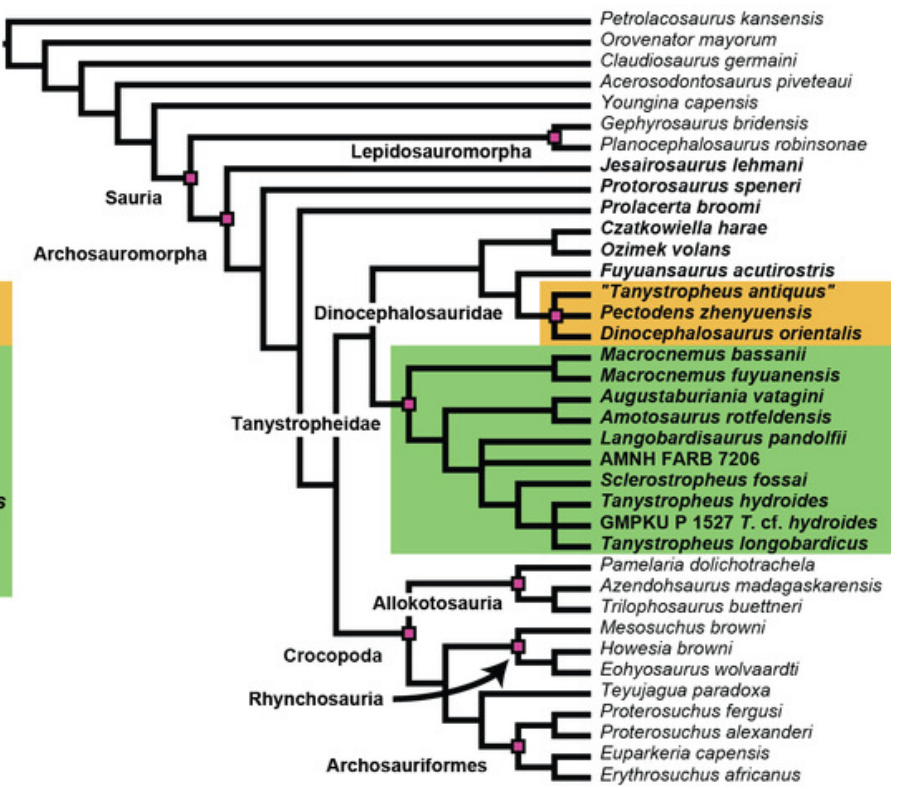




\title{
Figure 36
}

\author{
Results of analysis 4 (ratio characters included and specified characters ordered; \\ Czatkowiella harae, Tanystropheus "conspicuus", and "Tanystropheus antiquus" pruned \\ a priori).
}

(A) Strict consensus tree out of 154 MPTs with 1241 steps. Bremer values above 1 and Bootstrap frequencies above $50 \%$ are provided above and below each node, respectively. $(B)$ Reduced strict consensus tree after the a posteriori exclusion of Orovenator mayorum and Elessaurus gondwanoccidens. OTUs formerly considered to be "protorosaurs" are highlighted in bold.

A

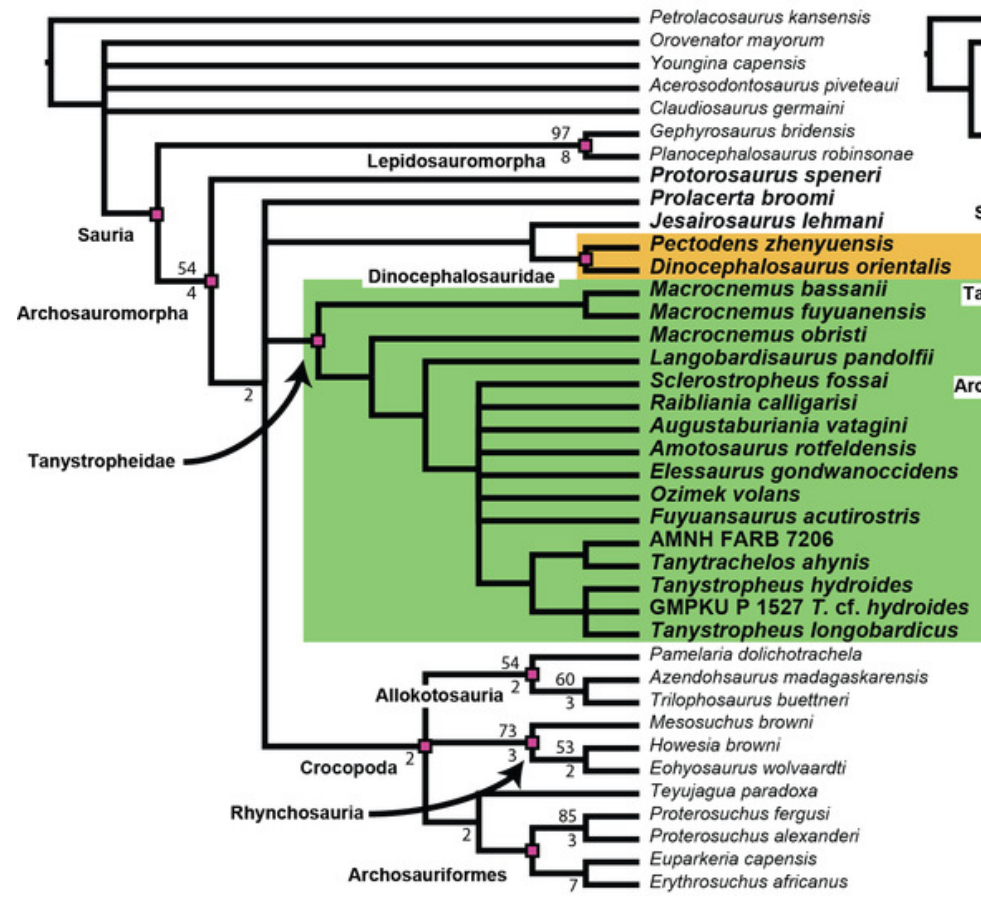

B

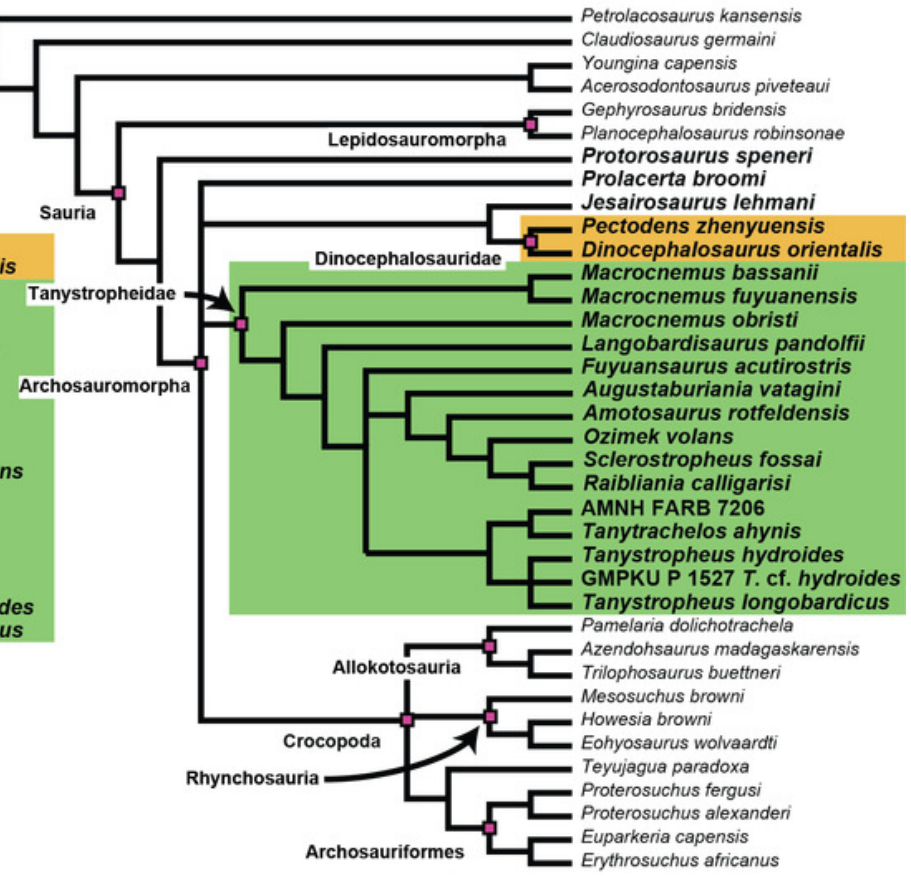




\section{Figure 37}

Time-calibrated phylogenetic tree based on the relationships recovered in analysis 4 .

The topology of the SCT is figured for Sauria, whereas the topology of the RSCT (excluding a posteriori the early Permian Orovenator mayorum) is used to indicate the relationships between the late Permian non-saurian diapsids. The black boxes indicate the possible temporal range of each OTU based on the available stratigraphic information (e.g., Pectodens zhenyuensis is known only from Member II of the Guanling Formation, which cannot be further restricted than being of Anisian age, and therefore the possible temporal range of Pectodens zhenyuensis covers the complete Anisian). The non-saurian diapsid taxa Petrolacosaurus kansensis and Orovenator mayorum from the Carboniferous and early Permian, respectively, are not indicated to since they are considerably older than the other taxa of this study. Their phylogenetic position is indicated in Figure 36a. The timescale based on the International Chronostratigraphic Chart of the International Commission on Stratigraphy (accessed May 2020). 


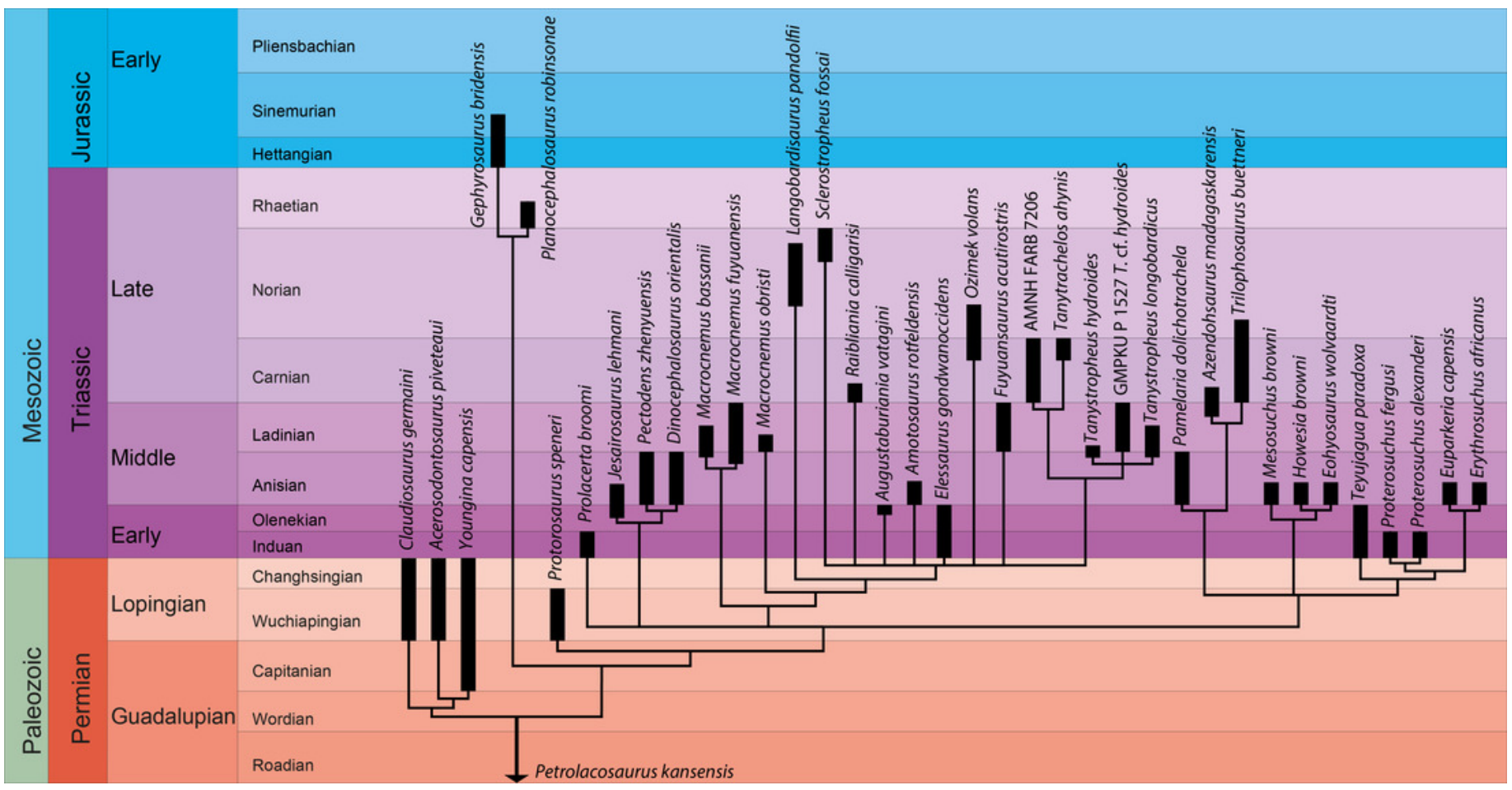

